Examens de l'OCDE sur la gouvernance publique

Revue du système de passation des marchés publics en Algérie

VERS UN SYSTÈME EFFICIENT, OUVERT ET INCLUSIF 



\section{Revue du système de passation des marchés publics en Algérie}

VERS UN SYSTÈME EFFICIENT, OUVERT ET INCLUSIF 
Ce document, ainsi que les données et cartes qu'il peut comprendre, sont sans préjudice du statut de tout territoire, de la souveraineté s'exerçant sur ce dernier, du tracé des frontières et limites internationales, et du nom de tout territoire, ville ou région.

Les données statistiques concernant Israël sont fournies par et sous la responsabilité des autorités israéliennes compétentes. L'utilisation de ces données par l'OCDE est sans préjudice du statut des hauteurs du Golan, de Jérusalem-Est et des colonies de peuplement israéliennes en Cisjordanie aux termes du droit international.

Merci de citer cet ouvrage comme suit :

OCDE (2019), Revue du système de passation des marchés publics en Algérie : Vers un système efficient, ouvert et inclusif,

Examens de l'OCDE sur la gouvernance publique, Éditions OCDE, Paris, https://doi.org/10.1787/49802cd0-fr.

ISBN 978-92-64-32691-0 (imprimé)

ISBN 978-92-64-44995-4 (pdf)

Examens de l'OCDE sur la gouvernance publique

ISSN 2226-5953 (imprimé)

ISSN 2226-5961 (en ligne)

Crédits photo: Couverture $\odot$ Leonid Andronov/Shutterstock.com.

Les corrigenda des publications sont disponibles sur : www.oecd.org/about/publishing/corrigenda.htm.

(C) OCDE 2019

L'utilisation de ce contenu, qu'il soit numérique ou imprimé, est régie par les conditions d'utilisation suivantes : http://www.oecd.org/fr/conditionsdutilisation. 


\section{Avant-propos}

Représentant plus de $20 \%$ du PIB en Algérie, les marchés publics ont un impact considérable sur les finances publiques et sur la qualité des services publics offerts aux citoyens. Ce volume élevé est lié aux politiques d'investissement public depuis la fin des années 1990, notamment dans l'éducation, la santé, les infrastructures de transport et l'énergie.

Avec une population composée majoritairement de jeunes, une espérance de vie qui se prolonge, des besoins en infrastructures croissants dus à la taille du pays, les marchés publics sont de plus en plus considérés comme un pilier fondamental de la gouvernance stratégique du pays.

L'OCDE aide les pays à améliorer leurs systèmes de passation des marchés publics en se basant sur la Recommandation du Conseil de l'OCDE sur les marchés publics de 2015 et d'autres standards internationaux. Le gouvernement algérien représenté par le ministère des Finances a demandé en 2017 le soutien de l'OCDE afin d'apporter un diagnostic complet du système de passation des marchés publics. Ce diagnostic a pour but d'aider les gouvernements à concevoir et mettre en place des politiques stratégiques basées sur des bonnes pratiques internationales tout en prenant en considération le contexte de chaque pays. Cette "Revue du système de passation des marchés publics en Algérie, Vers un système efficient, ouvert et inclusif » identifie les forces et les faiblesses du système algérien et propose des recommandations sur mesure.

Pour répondre aux défis majeurs que connaît l'Algérie, la mise en place d'un système de passation des marchés publics efficace, efficient et intègre s'impose comme une nécessité. Conscients de ces défis, les autorités algériennes ont entamé une série de réformes visant à améliorer le système en place. Cependant, il reste encore de nombreux chantiers à entamer afin que le système soit en ligne avec les bonnes pratiques internationales, notamment par la mise en place effective de l'Autorité de régulation des marchés publics et du système électronique de passation des marchés publics.

L'amélioration et la refonte du système nécessiteront une action collective basée sur une compréhension claire des priorités et des responsabilités. Seul un effort coordonné de l'ensemble des parties prenantes et du secteur public permettra au système de passation des marchés publics d'atteindre les objectifs d'efficacité et de bonne utilisation des fonds publics prévus dans le cadre réglementaire. Au-delà des efforts relatifs au cadre réglementaire et institutionnel, le renforcement des capacités des principaux acteurs est aussi une pièce maîtresse de la refonte du système.

Cette étude fait partie d'une série examens de la gouvernance publique de l'OCDE sur la passation des marchés publics, et a été mené dans le cadre d'un partenariat avec la Commission européenne, pour accompagner l'Algérie dans le renforcement du système de passation des marchés publics.

Le document [GOV/PGC/ETH(2019)5] a été approuvé par le groupe de travail des experts des marchés publics de l'OCDE le 06 septembre 2019 et a été déclassifié par le Comité de la gouvernance publique le 18 octobre 2019 et préparé pour publication par le Secrétariat de l'OCDE.

Le présent document a été élaboré avec l'aide financière de l'Union européenne (UE). Les opinions qui y sont exprimées ne doivent en aucun cas être considérées comme reflétant la position officielle de l'UE. 


\section{Remerciements}

Sous la direction et la supervision de Marcos Bonturi, Directeur de la gouvernance publique, de Janos Bertók, Responsable de la division de l'intégrité dans le secteur public et de Paulo Magina, Responsable de l'unité des marchés publics, ce rapport a été rédigé et coordonné par Kenza Khachani, Analyste des politiques au sein de l'unité des marchés publics. Les autres auteurs sont Antoine Comps, Minjoo Son et Yoann Untereiner de l'unité des marchés publics. Angelos Binis et Guilio Nessi ont également apporté leurs contributions et commentaires. Mr Amar Aouidef, a assuré la coordination locale du projet.

Le rapport a été préparé pour publication par Meral Gedik. Isabelle Delpech et Andrea Uhrhammer ont apporté un soutien rédactionnel. Rania Haidar, Laura Roberts et Aleksandra Bogusz ont fourni l'assistance administrative.

L'OCDE exprime sa gratitude au gouvernement algérien, au Ministère des Finances et aux institutions gouvernementales impliquées dans ce rapport, ainsi qu'aux organisations de la société civile rencontrées durant les missions exploratoires. En particulier, l'OCDE souhaite remercier Mr Mohamed Loukal, Ministre en charge des Finances, Mr Mohamed Bouzerde, Chef de la Division des Marchés Publics (Ministère des Finances), Mme Zheira Mezghrani, Directrice du Contrôle de la Régularité des Marchés Publics (Ministère des Finances) ; Mr Omar Ladjel, Sous-Directeur de la Réglementation au sein de la Division des Marchés Publics (Ministère des Finances). Par ailleurs, l'OCDE souhaite remercier toutes les entités publiques, les représentants du secteur privé et la société civile qui ont aidé à la rédaction de ce rapport que ce soit à Alger, ou en région (Blida et Oran). Ce rapport a également bénéficié des contributions de pairs : $\mathrm{Mr}$ Philippe Maraval, Directeur des Achats Groupe, Pôle Emploi, France et Mr Farid Yaker, Chef de Programme au Programme des Nations-Unies pour l'Environnement.

L'OCDE exprime également sa gratitude à la Commission Européenne et à la délégation de l'Union Européenne à Alger pour leur soutien financier. En particulier à Mr John O'Rourke, Ambassadeur/Chef de Délégation de l'Union européenne en Algérie et à Mr Zé Alves Pereira, Attaché Économie et Finances. Les remerciements vont aussi à SIGMA, une initiative conjointe de l'OCDE et de la Commission Européenne, en particulier Mr Gorecki Piotr-Nils, Conseiller principal pour son soutien. 


\section{Table des matières}

$\begin{array}{ll}\text { Avant-propos } & 3\end{array}$

$\begin{array}{lr}\text { Remerciements } & 4\end{array}$

$\begin{array}{ll}\text { Abréviations et acronymes } & 8\end{array}$

Résumé 11

1 Faciliter l'accès et la participation au système de passation des marchés publics en Algérie

1.1. Un cadre réglementaire et institutionnel stable et cohérent pour les marchés publics $\quad 15$

1.2. Promouvoir les procédures concurrentielles et limiter le recours aux exceptions 25

1.3. Des processus et une documentation favorisant la concurrence 39

$\begin{array}{ll}\text { Propositions d'action } & 45\end{array}$

$\begin{array}{ll}\text { Références } & 46\end{array}$

2 Préserver l'intégrité du système de passation des marchés publics en Algérie $\quad 51$

2.1. Renforcer le système d'intégrité dans les marchés publics $\quad 55$

2.2. Développer et entretenir une culture d'intégrité dans les marchés publics 61

2.3. Appliquer des dispositifs de surveillance et de contrôle en intégrant des stratégies de management des risques dans les marchés publics $\quad 72$

Propositions d'action $\quad 83$

$\begin{array}{ll}\text { Références } & 85\end{array}$

3 Transparence et dématérialisation du système de passation des marchés publics en Algérie

3.1. Assurer un degré adéquat de transparence dans le système de passation des marchés publics

3.2. Développer un système de passation électronique des marchés publics : un élément clé pour améliorer l'efficience du système 96

Propositions d'action 106

$\begin{array}{lr}\text { Références } & 107\end{array}$

4 Optimiser les marchés publics en Algérie pour une plus grande efficience 109

4.1. L'évaluation des besoins et l'analyse des marchés, une étape clé pour la performance du système de passation des marchés publics

4.2. Des documents d'appel d'offres adaptés à la réalité du marché 122

4.3. Mettre en place des outils pour améliorer l'efficacité de la passation des marchés 128 
4.4. Renforcer l'évaluation du système de passation des marchés publics 134

Propositions d'action $\quad 142$

$\begin{array}{ll}\text { Références } & 144\end{array}$

5 La contribution des marchés à la réalisation des objectifs des politiques publiques en Algérie

5.1. Développer une stratégie pour la prise en compte des politiques publiques dans les marchés publics

5.2. Définir un cadre pour la prise en compte des politiques publiques dans les marchés publics 156

5.3. Les marchés publics, un marché considérable pour le développement des PME

161

5.4. Mesures d'accompagnement pour une implémentation efficiente de politiques publiques

dans les marchés publics

168

Propositions d'action

172

Références

173

\section{Le renforcement des capacités, un prérequis au bon fonctionnement du système} de passation des marchés publics en Algérie

6.1. Pour le développement d'une stratégie nationale de renforcement des capacités dans le domaine des marchés publics

6.2. Mener des actions concrètes pour le renforcement des capacités des acteurs du système de passation des marchés publics

Références

\section{GRAPHIQUES}

Graphique 1.1. Les fonctions principales du cadre institutionnel du système de passation des marchés publics 20

Graphique 1.2. Avantages de la concurrence sur les marchés publics 26

Graphique 1.3. Hiérarchie des procédures de passation des marchés publics 27

Graphique 1.4. Les différentes procédures de passation des marchés publics en Algérie 28

Graphique 2.1.La Recommandation du Conseil de l'OCDE sur l'intégrité publique 53

Graphique 2.2.Les facteurs problématiques pour faire des affaires en Algérie (2015-16) 54

Graphique 2.3. Indice de perception de la corruption (2017)

Graphique 2.4.Acteurs à impliquer dans le développement d'une stratégie de lutte contre la corruption dans le domaine des marchés publics

Graphique 2.5. Le système de contrôles appliqués aux marchés publics en Algérie 72

Graphique 2.6. Le modèle des trois lignes de maîtrises du contrôle interne 73

Graphique 2.7. Risques d'atteinte à l'intégrité dans le processus de passation des marchés 77

Graphique 2.8.Cycle de management des risques selon les standards internationaux (ISO 31000:2018) 78

Graphique 3.1. Évolution des systèmes d'e-procurement

Graphique 3.2. Les éléments nécessaires à la mise en place d'une stratégie de passation électronique des

marchés publics

Graphique 3.3. Fonctionnalités des systèmes de passation de marchés électroniques dans les pays de l'OCDE

Graphique 3.4. Intégration verticale et horizontale du système de passation électronique des marchés publics 104

Graphique 4.1. Approche traditionnelle et stratégique des marchés publics 111

Graphique 4.2. L'impact de l'analyse des besoins et de l'analyse du marché sur l'appel d'offres 112

Graphique 4.3. Taux d'inflation dans les pays de l'OCDE et en Algérie (2007-17) 127

Graphique 4.4. Différents niveaux d'agrégation de la demande 129

Graphique 4.5. L'existence de centrales d'achats dans les pays de l'OCDE 130

Graphique 4.6. Accords-cadres : les étapes de la définition des catégories d'achat 133

Graphique 4.7. Les trois niveaux pour mesurer la performance du système de passation des marchés publics 135

Graphique 5.1. Différentes façons de prendre en compte les ODD dans les marchés publics 151

Graphique 5.2. Les objectifs de développement durables 
Graphique 5.3. Coordination entre les entités chargées du système de passation des marchés publics et celles chargées des politiques publiques

Graphique 5.4. Les éléments à prendre en compte pour insérer le développement durable et l'innovation dans le cadre réglementaire des marchés publics

Graphique 5.5. Environnement d'affaires des PME : un cadre conceptuel

Graphique 5.7. Pays qui encouragent l'allotissement des marchés

Graphique 5.8. Pourcentage des pays qui mesurent l'impact du recours aux marchés publics

Graphique 6.1. Les parties prenantes au développement de la stratégie nationale de renforcement des

capacités dans les marchés publics en Algérie

Graphique 6.2. Les acteurs du système de passation des marchés publics en Algérie

Graphique 6.3. La reconnaissance du métier d'acheteur public comme un métier spécifique dans les pays membres de l'OCDE

\section{TABLEAUX}

Tableau 1.1. Articles du DMP nécessitant des décrets exécutifs ou des arrêtés 16

Tableau 1.2. Utilisation de la méthode RACI pour la réforme des marchés publics à Malte 25

Tableau 1.3. Seuils des procédures formalisées dans l'UE 30

Tableau 1.4. Les exceptions à l'appel d'offres en Algérie 32

Tableau 1.5. Délais de soumission constatés pour les appels d'offres en Algérie 42

Tableau 2.1. Les seuils de compétence des commissions des marchés par types de marchés 75

Tableau 2.2. Principales sanctions pénales en relation avec les activités de marchés publics 79

Tableau 2.3. Les fautes professionnelles et les sanction disciplinaires applicables dans la fonction publique en Algérie

Tableau 3.1. Obligation de transparence lors des procédures de passation des marchés publics en Algérie

Tableau 4.1. Inconvénients de l'utilisation du seul critère du prix pour la sélection des offres

Tableau 4.2. Lignes directrices pour l'attribution des marchés de fournitures au Mexique

Tableau 4.3. Les quatre catégories d'accords-cadres

Tableau 4.4.Catégories de biens et services fréquemment couverts par les accords-cadres de l'UE 


\section{Abréviations et acronymes}

\begin{tabular}{|c|c|}
\hline AMP & Accord multilatéral sur les marchés publics \\
\hline ANEP & Agence nationale d'édition et de publication \\
\hline Ansej & Agence nationale de soutien à l'emploi des jeunes \\
\hline APAC & Achats publics avant commercialisation \\
\hline ARMP & Autorité de régulation des marchés publics \\
\hline BOAMP & Bulletin officiel des annonces des marchés publics \\
\hline Bomop & Bulletin officiel des marchés de l'opérateur public \\
\hline $\mathrm{CACl}$ & Chambre algérienne de commerce et d'industrie \\
\hline CAD & Dollar canadien \\
\hline CCAG & Cahier des clauses administratives générales \\
\hline CNED & Caisse nationale d'équipement pour le développement \\
\hline CNUCC & Convention des Nations Unies contre la corruption \\
\hline CPV & Common procurement vocabulary, ou vocabulaire commun pour les marchés publics \\
\hline CTRF & Cellule de traitement du renseignement financier \\
\hline DAJ & Direction des affaires juridiques \\
\hline DESS & Diplôme d'études supérieures spécialisées \\
\hline DUME & Document unique de marché européen \\
\hline DZD & Dinars algériens \\
\hline EPE & Entreprise publique économique \\
\hline EPIC & Établissement public à caractère industriel et commercial \\
\hline EUR & Euro \\
\hline GBP & Livre sterling \\
\hline GMC & Modèles de contrat du gouvernement néo-zélandais \\
\hline GME & Groupement momentané d'entreprise \\
\hline IGF & Inspection générale des finances \\
\hline Inapi & Institut national algérien de la propriété intellectuelle \\
\hline ISGP & Institut supérieur de gestion et de planification \\
\hline JORADP & Journal officiel de la république algérienne démocratique et populaire \\
\hline KRW & Won sud-coréen \\
\hline MBIE & Ministère du Commerce, de l'Innovation et du Travail de Nouvelle-Zélande \\
\hline MEER & Ministère de l'Environnement et des Énergies renouvelables \\
\hline MENA & Moyen-Orient et Afrique du Nord \\
\hline MOOC & MOOC (Massive Open Online Course, formation en ligne ouverte à tous) \\
\hline MPTTN & Ministère de la Poste, des Télécommunications, des Technologies et du Numérique \\
\hline MXN & Peso mexicain \\
\hline OCDE & Organisation de coopération et de développement économiques \\
\hline OCRC & Office central de répression de la corruption \\
\hline ODD & Objectifs de développement durable \\
\hline OGP & Bureau des achats du gouvernement d'Irlande \\
\hline OMC & Organisation mondiale du commerce \\
\hline ONPLC & Organe national de prévention et de lutte contre la corruption \\
\hline ONS & Office national des statistiques \\
\hline OPP & Objectifs de politiques publiques \\
\hline
\end{tabular}




\begin{tabular}{|l|l|}
\hline PCD & Plan communal de développement \\
\hline PIB & Produit intérieur brut \\
\hline PME & Petites et moyennes entreprises \\
\hline PNUD & Programme des Nations Unies pour le développement \\
\hline PPI & Partenariats publics d'innovation \\
\hline PPP & Partenariat public privé \\
\hline PSC & Programme sectoriel centralisé \\
\hline PSD & Programme sectoriel déconcentré \\
\hline RIME & Répertoire interministériel des métiers de l'État \\
\hline RSE & Responsabilité sociétale des entreprises \\
\hline SAD & Système d'acquisition dynamique \\
\hline SGFP & Statut général de la fonction publique \\
\hline TND & Dinar tunisien \\
\hline UE & Union européenne \\
\hline UNODC & Office des Nations Unies contre la drogue et le crime \\
\hline UNSPSC & United Nations Standard Products and Services Code, ou code de classification des produits et services des Nations Unies \\
\hline
\end{tabular}





\section{Résumé}

Avoisinant un PIB de plus 180 milliards d'euros en 2018, l'économie algérienne est l'une des plus importantes d'Afrique du Nord. En effet, les marchés publics représentent plus de $20 \%$ du PIB en Algérie, bien au-delà de la moyenne des pays de l'OCDE qui représentent près de $12 \%$ du PIB en 2017 . Compte tenu du volume important que représente ces dépenses, la bonne gestion des marchés publics peut et doit contribuer au renforcement de l'efficience du secteur public.

\section{Principales conclusions}

Les récentes modifications apportées au cadre réglementaire régissant les marchés publics (Décret présidentiel $\mathrm{n}^{\circ} 15-247$ du 16 septembre 2015, ou DMP) ont permis de réaliser des avancées considérables, mais des efforts supplémentaires sont nécessaires pour que le système réglementaire et institutionnel soient cohérents, stables et durables. Ainsi, malgré l'entrée en vigueur du DMP en décembre 2015, l'Autorité de régulation des marchés publics (ARMP), en charge des fonctions centrales de base et complémentaires, n'a pas encore été mise en place. Par ailleurs, un certain nombre de mécanismes et dispositifs devraient être revus afin d'assurer un degré de concurrence adéquat.

Des obligations de transparence sont prévues dans le DMP, cependant elles ne couvrent pas toutes les étapes du cycle de passation des marchés publics. Le respect des obligations de transparences et l'accès de l'information à toutes les parties prenantes sont à améliorer. Un outil fondamental pour améliorer la transparence et l'efficacité du système est la mise en place d'un système de passation électronique des marchés publics que le pays se prépare à acquérir.

L'intégrité dans les marchés publics est un élément essentiel du cadre réglementaire, en particulier du DMP qui énonce des mécanismes afin de lutter contre la corruption. Cependant certains d'entre eux doivent toujours être mis en place ou largement déployés tels que le développement du code de conduite. Par ailleurs, les politiques en place se focalisent principalement sur des mesures de détection et de sanction et non pas de prévention. L'approche coordonnée et stratégique pour préserver l'intégrité dans les marchés publics pourrait être améliorée, y compris pour le système de surveillance et de contrôle.

Le DMP a introduit certains des outils visant à améliorer l'efficience du système de passation des marchés publics. Cependant, ces derniers sont peu utilisés dans la pratique et d'autres outils gagneraient à être intégrés dans le cadre réglementaire. L'insuffisance de l'évaluation des besoins, des études de marchés et de dialogue structuré avec les opérateurs économiques sont des freins à l'amélioration de l'efficacité du système. Enfin, l'absence du système de passation électronique des marchés publics limite la collecte des données sur la performance du système et leur suivi.

En outre, les marchés publics ont été identifiés comme un levier stratégique pour atteindre des objectifs de politiques publiques. Le recours au marchés publics pour atteindre des objectifs de développement durable, d'innovation ou de conduite responsable des entreprises reste insuffisant. Par ailleurs, la cohérence entre les politiques nationales et celles relatives aux marchés publics sont à améliorer. 
Pour finir, le niveau de capacité des parties prenantes au système de passation des marchés publics joue un rôle central dans l'amélioration du système. En Algérie, les capacités insuffisantes des acteurs clés du système de passation des marchés publics et les actions limitées de renforcement des capacités sont un obstacle majeur au bon fonctionnement de l'ensemble du système.

\section{Principales recommandations}

- L'Algérie gagnerait à mettre en place l'ARMP dans les plus brefs délais, afin d'assurer le bon fonctionnement du système et la cohérence de son cadre réglementaire et institutionnel. Pour améliorer l'accès aux marchés publics, le ministère des Finances gagnerait à revoir les systèmes d'exceptions qui justifient le non-recours aux appels d'offres, et à encadrer davantage les procédures de passation des marchés des entités non soumises au DMP, telles que les entreprises publiques économiques.

- Les obligations de transparence gagneraient à couvrir tout le cycle de passation des marchés publics. Le déploiement progressif d'un système électronique de passation des marchés publics permettrait d'améliorer la transparence et de collecter les données visant à évaluer la performance du système. Dans le cadre de la mise en place de son système électronique, l'Algérie gagnerait à développer une stratégie spécifique et à impliquer l'ensemble des parties prenantes à travers un comité de pilotage.

- L'Algérie pourrait adopter une stratégie d'intégrité publique avec des objectifs clairs à atteindre, en prenant en compte les spécificités des risques inhérents aux marchés publics. En outre, afin d'assurer des normes d'intégrité communes en matière de marchés publics, l'Algérie devrait adopter un code d'éthique et de déontologie pour les agents publics impliqués dans les marchés publics. Le pays devrait également accroître l'efficacité du système de contrôles appliqués aux marchés publics en établissant un système effectif des trois lignes de maitrise.

- Pour améliorer l'efficacité du système de passation des marchés publics, l'Algérie gagnerait à renforcer les études de marché conduites par les services contractants et à mettre en place des mécanismes de dialogue formalisés avec le secteur privé. Par ailleurs, le pays gagnerait à revoir et à mettre en place des outils visant à améliorer l'efficacité et l'efficience du système de passation des marchés publics.

- L'Algérie gagnerait à utiliser davantage les marchés publics comme un levier stratégique pour la mise en place des différentes politiques publiques en particulier en termes de développement durable, d'innovation et de responsabilité sociétale des entreprises en cohérence avec les politiques nationales. Cela ne peut se faire sans le développement d'une vision stratégique.

- L'Algérie devrait développer une stratégie nationale pour le renforcement des capacités dans les marchés publics. Au préalable, l'identification des agents en charge des marchés publics et des autres acteurs du système est nécessaire. En plus de la reconnaissance du métier d'acheteur comme une profession spécifique et du développement d'un cadre de compétence, l'Algérie devrait renforcer son offre de formation et mettre à disposition des acteurs du système des outils de renforcement des capacités. 


\section{Faciliter l'accès et la participation au système de passation des marchés publics en Algérie}

Le cadre institutionnel et réglementaire du système de passation des marchés publics en Algérie connaît de nombreux défis. Ce chapitre analyse le système en place et fournit des recommandations au gouvernement algérien pour renforcer sa cohérence et sa stabilité. II traite également de l'accès aux marchés publics des soumissionnaires potentiels de toutes tailles et de tous horizons, et présente des recommandations visant à améliorer la concurrence dans les marchés publics et, par là-même, leur efficacité. 
Les marchés publics permettent de fournir des services et infrastructures essentiels aux citoyens et d'assurer le bon fonctionnement du secteur public. Les gouvernements utilisent de plus en plus les marchés publics comme un levier pour atteindre des objectifs stratégiques, y compris améliorer l'efficacité des services publics, tout en tenant compte des pressions budgétaires (OCDE, 2018[1] $)$.

Comme mentionné dans la Recommandation du Conseil de l'OCDE sur les marchés publics, un système de passation des marchés publics efficace, efficient et intègre repose sur un accès équitable aux opportunités de marchés publics pour les fournisseurs potentiels de toutes tailles. L'accès aux marchés publics est assuré par:i) des cadres cohérents et stables sur le plan institutionnel, législatif et réglementaire, aussi clairs et simples que possible; ii) des documentations d'appel d'offres claires, synthétiques, normalisées et adaptées aux besoins; ainsi que iii) l'utilisation de procédures concurrentielles et le recours limité aux exceptions et à la sollicitation d'une source unique (OCDE, 2015[2]). Par ailleurs, cet accès est également lié à d'autres principes de la Recommandation du Conseil de l'OCDE sur les marchés publics(voir encadré 1.1).

\section{Encadré 1.1. Liens entre le principe "Accès » et les autres principes de la Recommandation du Conseil de l'OCDE sur les marchés publics}

- Transparence : La transparence contribue à un traitement juste et équitable des soumissionnaires.

- Intégrité : L'accès aux opportunités de marchés publics pour les concurrents de toutes tailles, donc la limitation du recours aux exceptions à la mise en concurrence (attribution directe, procédures accélérées, etc.), favorise l'obtention d'un meilleur rapport qualité/prix et diminue les risques de corruption.

- Participation : La conception des appels d'offres doit permettre d'encourager une vaste participation de soumissionnaires, y compris de nouveaux entrants et des petites et moyennes entreprises (PME). De plus, un dialogue transparent et régulier avec les fournisseurs et les associations professionnelles permet une meilleure compréhension des besoins du gouvernement, facilitant ainsi l'accès aux opportunités de marchés pour l'ensemble des fournisseurs.

- Efficience : Des cadres institutionnels, légaux et réglementaires stables et cohérents sont essentiels pour l'augmentation de la participation aux marchés publics ; ce sont également des éléments fondamentaux pour assurer un système de passation des marchés publics durable et efficace. L'utilisation de procédures de mise en concurrence permet de mener à l'efficience en obtenant le meilleur rapport qualité/prix).

- Systèmes électroniques de passation des marchés publics : ils assurent un meilleur accès aux opportunités de marchés, augmentant ainsi la concurrence. De plus, les autorités de la concurrence peuvent utiliser les informations issues du système électronique de passation des marchés pour détecter les stratégies de soumissions suspectes, qui peuvent être symptomatiques d'ententes collusoires.

- Capacité : Des agents chargés de la passation des marchés publics sont formés pour assurer la fourniture d'une documentation d'appel d'offres claire et cohérente, si possible standardisée.

- Évaluation : Les règles justifiant et autorisant le recours à des exceptions aux procédures de mise en concurrence doivent être compréhensibles et claires, car de telles exceptions limitent la concurrence.

Source : (OCDE, 2016[3]). 
L'accès au système de passation des marchés publics et son efficacité reposent également sur la participation des différentes parties prenantes, y compris lors de l'apport de modifications au système. Cette participation passe par une approche inclusive et collaborative, et un dialogue global sur le système et ses objectifs. (OCDE, 2015[2])

L'accès aux marchés publics est un défi majeur en Algérie, où ils représentent plus de $20 \%$ du produit intérieur brut (PIB). Les récentes modifications apportées au cadre réglementaire ont certes permis d'améliorer le niveau de concurrence et l'accès aux marchés publics, cependant, des efforts supplémentaires sont nécessaires pour que ce système soit conforme aux bonnes pratiques internationales.

Ce chapitre analyse le système en place, le niveau d'accès aux opportunités des marchés publics et le niveau de participation globale de différentes parties prenantes. II propose des recommandations visant à améliorer l'accès au système, et donc son efficacité.

\subsection{Un cadre réglementaire et institutionnel stable et cohérent pour les marchés publics}

Un système de passation des marchés publics avec un cadre institutionnel et réglementaire cohérent et stable est crucial pour assurer son efficacité et élargir la participation aux marchés publics. Les bonnes pratiques internationales soulignent la nécessité de disposer d'un cadre institutionnel aussi clair et simple que possible, et dont les exigences n'entrent pas en conflit avec d'autres textes réglementaires (OCDE, 2015[2]).

En Algérie, le cadre réglementaire repose principalement sur le décret présidentiel $n^{\circ} 15-247$ du 16 septembre 2015 (DMP) ainsi que sur plusieurs arrêtés et décrets exécutifs. Le cadre institutionnel prévu dans le DMP consacre un rôle clé à l'Autorité de régulation des marchés publics (ARMP), bien qu'elle n'ait pas encore été mise en place. En attendant, c'est la Division des marchés publics du ministère des Finances qui assure le fonctionnement et la cohérence du système.

\subsubsection{Clarifier le cadre réglementaire applicable aux marchés publics algériens}

Un cadre réglementaire stable et cohérent est primordial pour un système de passation des marchés publics efficace et pérenne. Ce cadre réglementaire doit notamment être clairement organisé et accessible facilement et gratuitement. En Algérie, le système de passation des marchés publics est régi par le DMP, entré en vigueur le 20 décembre 2015 en remplacement du décret présidentiel $\mathrm{n}^{\circ} 10-236 \mathrm{du}$ 7 octobre 2010, qu'il abroge. Le DMP en reprend de nombreuses dispositions, tout en faisant évoluer le droit des marchés publics algériens sur plusieurs points, conformément aux bonnes pratiques internationales.

\section{Assurer un cadre réglementaire clair, complet et accessible en ligne}

Le cadre réglementaire régissant les marchés publics doit être clair, disponible en ligne et inclure toutes les réglementations qui complètent et détaillent certaines dispositions, telles que les décrets exécutifs, arrêtés, et circulaires. Ceci est nécessaire pour assurer une compréhension commune du secteur public et du secteur privé des objectifs que veut atteindre l'État à travers les marchés publics. Par ailleurs, il s'agit d'un prérequis pour améliorer la participation des opérateurs économiques aux marchés publics (OCDE, 2016[3]).

En outre, le cadre réglementaire régissant les marchés publics doit être stable, cohérent et facilement applicable. Les textes d'application réglementaires (arrêtés ou décrets exécutifs en Algérie) peuvent 
néanmoins être revus régulièrement afin d'adapter les modalités détaillées de passation des marchés publics aux évolutions du marché (EBRD, 2009[4]).

Le DMP renvoie à plusieurs arrêtés ou décrets exécutifs du ministre en charge des Finances, dont la plupart ont été adoptés avant l'entrée en vigueur du DMP en 2015. De nombreux articles du DMP sont indirectement non applicables (ou difficilement applicables) car ils stipulent que "les modalités d'application du présent article seront précisées en tant que besoin par arrêté du ministre chargé des Finances ". Cette disposition particulière crée un flou juridique car les articles visés ne sont pas suffisamment complets ou précis. Les discussions menées par l'équipe de l'Organisation de coopération et de développement économiques (OCDE) lors des missions exploratoires en Algérie ont d'ailleurs mis en lumière un réel besoin pour différentes parties prenantes de préciser les dispositions du DMP. Cependant ce besoin n'a pas été rapporté à la Division des marchés publics chargée de ces aspects.

Certains arrêtés ou décrets exécutifs n'ont toujours pas été mis à jour car ils sont relatifs à l'ancien cadre réglementaire, le décret présidentiel $n^{\circ} 10-236$ du 7 octobre 2010 portant réglementation des marchés publics. L'article 218 du DMP précise qu'ils restent en vigueur jusqu'à la publication des textes pris en application des dispositions du DMP. D'autres arrêtés ou décrets exécutifs qui permettent de préciser certaines dispositions du DMP « en cas de besoin » n'ont jamais été élaborés et publiés, notamment ceux mentionnés aux articles 26, 84 et 107.

Cependant, le principal problème provient de l'absence de mise en œuvre, sur une durée de plusieurs années consécutives, de dispositions essentielles du DMP, et notamment des articles 203 (portail électronique des marchés publics) et 213 (ARMP) du DMP. Comme souligné dans plusieurs chapitres du présent rapport, l'absence de mise en place de ces dispositions, en particulier celles relatives au cadre institutionnel, nuit à la clarté du système. Par ailleurs, certaines de ces dispositions sont liées à d'autres parties du cadre réglementaire, ce qui rend leur mise en place effective impossible ou partielle. Le tableau 1.1 ci-dessous donne plus de détails sur les articles concernés. II dresse la liste des articles du DMP qui sont partiellement non applicables, indirectement non applicables ou directement non applicables du fait de l'absence de décret ou d'arrêté d'application.

\section{Tableau 1.1. Articles du DMP nécessitant des décrets exécutifs ou des arrêtés}

\begin{tabular}{l|c|c|c|c}
\hline & $\begin{array}{c}\text { Nécessite des précisions } \\
\text { via un arrêté du } \\
\text { ministère des Finances }\end{array}$ & $\begin{array}{c}\text { Nécessite un décret } \\
\text { exécutif }\end{array}$ & $\begin{array}{c}\text { Nécessite des précisions } \\
\text { via un arrêté du } \\
\text { ministère des Finances }\end{array}$ & $\begin{array}{c}\text { Nécessite que le DMP } \\
\text { soit pleinement appliqué }\end{array}$ \\
\hline $\begin{array}{l}\text { Articles partiellement non } \\
\text { applicable }\end{array}$ & & & $12,164,200,201$ \\
\hline $\begin{array}{l}\text { Articles indirectement non } \\
\text { applicables }\end{array}$ & $84,107,125$ & 26 & $13,15,16,27,29,31,33$, & \\
\hline Articles non applicables & & & $34,35,41,45,46,47,49$, & $50,51,52,81,86,87$ \\
\hline
\end{tabular}

Source : DMP.

Lors des missions exploratoires de l'OCDE, plusieurs parties prenantes des secteurs public et privé ont fait part de difficultés à comprendre le système actuel. En effet, dans la pratique les différentes parties prenantes se retrouvent à utiliser un système binaire basé sur le nouveau cadre réglementaire pour certaines dispositions et sur l'ancien cadre réglementaire pour d'autres. La circulaire $n^{\circ} 003 / M F d u$ 22 novembre 2015 a clarifié la mise en œuvre de certaines dispositions du DMP en chevauchement avec le DMP précédent, mais cette circulaire n'était en application que jusqu'au 31 décembre 2018. Afin de clarifier le cadre réglementaire, le gouvernement algérien gagnerait à mettre en application les dispositions prévues dans le DMP et à préciser quelles dispositions sont officiellement suspendues en attendant leur mise en place effective. Par ailleurs, le gouvernement algérien gagnerait à actualiser les arrêtés relatifs à 
l'ancien cadre réglementaire et à publier les arrêtés complémentaires du ministre des Finances afin d'apporter plus de clarté et de cohérence au cadre réglementaire régissant les marchés publics.

Enfin, le cadre réglementaire régissant les marchés publics doit être publié et répertorié hiérarchiquement (OCDE, 2016[3] ). II devrait également être disponible sur une page web spécifique (voir chapitre 3). En l'absence du portail électronique des marchés publics prévu par le DMP, le ministère des Finances pourrait mettre en ligne une présentation hiérarchisée et exhaustive du cadre réglementaire des marchés publics (DMP et arrêtés d'application). Afin de clarifier l'application des différentes dispositions, cette présentation pourrait faire le lien entre les articles du DMP et les arrêtés d'application en vigueur, et indiquer le cas échant l'absence d'arrêté d'application.

\section{S’assurer du respect des grands principes de la commande publique pour tous les marchés publics des entités publiques}

Afin de clarifier la portée du cadre réglementaire régissant les marchés publics, il est généralement prévu des exemptions, exclusions et exceptions au cadre réglementaire principal (voir encadré 1.2).

\section{Encadré 1.2. Définition des exemptions, exclusions et exceptions dans le cadre des marchés publics}

- Exemptions : concernent le périmètre de l'application du cadre réglementaire principal, plus précisément comment ce cadre s'applique aux entités publiques (pouvoirs adjudicateurs, entreprises publiques, organisations non gouvernementales), y compris celles qui sont exemptées de l'obligation de le suivre strictement.

- Exclusions : la réglementation des marchés publics n'est pas appropriée à toutes les catégories d'achat. Certaines catégories - telles que l'immobilier, les contrats de travail, les dépenses militaires spécialisées ou l'aide internationale- nécessitent une législation ou une réglementation spécifique. Par conséquent, ces types de dépenses sont exclus de la réglementation principale sur les marchés publics.

- Exceptions : pour les dépenses publiques régies par la réglementation sur les marchés publics, des cas spéciaux peuvent nécessiter une procédure différente de la procédure d'appel d'offres par défaut. Les catégories d'achat, les motifs et les processus d'approbation et de contrôle sont codifiés dans la réglementation en tant que processus d'exception.

Source : (OCDE, à paraître $\left.{ }_{[5]}\right)$.

En ce qui concerne les exemptions, il existe des raisons valables pour qu'elles soient prévues dans le cadre réglementaire régissant les marchés publics. Par exemple, la nature même de l'activité de certaines entreprises publiques évoluant dans un environnement concurrentiel fait qu'elles ne peuvent pas suivre toutes les dispositions du cadre réglementaire principal régissant les marchés publics (OCDE, à paraître $\left[{ }_{[5]}\right)$. Les différences majeures entre le cadre principal régissant les marchés publics et les procédures d'achat des entreprises publiques concernent principalement les délais et les seuils d'appels d'offres ouverts. Ainsi, les États prévoient généralement dans leur cadre réglementaire des dispositions dites « d'exemption » pour ce type d'entreprises (OCDE, à paraître $[5])$. Par exemple, tous les pouvoirs adjudicateurs des pays de l'Union européenne(UE) doivent suivre la Directive européenne sur les marchés publics. Cependant une directive spécifique est prévue pour les marchés passés par les entités opérant dans les secteurs de l'eau, de l'énergie, des transports et des services postaux en raison des spécificités de ces secteurs. II existe par ailleurs des exemptions à cette directive pour les entreprises qui opèrent dans le secteur concurrentiel (Union Européenne, 2014[6]). II revient donc à chaque pays de mener une 
analyse détaillée afin de trouver un équilibre entre la flexibilité requise pour les entités opérant dans un secteur concurrentiel et l'intégrité et la transparence du système (OCDE, à paraître $\left.{ }_{[5]}\right)$.

Le cadre réglementaire algérien régissant les marchés publics est soumis aux principes de liberté d'accès à la commande publique, d'égalité de traitement des candidats et de transparence des procédures (art. 5 du DMP). Les entités soumises au DMP sont:

1. l'État,

2. les collectivités territoriales,

3. les établissements publics à caractère administratif,

4. les établissements publics soumis à la législation régissant les activités commerciales, lorsque ceux-ci sont chargés de la réalisation d'une opération financée, totalement ou partiellement, sur concours temporaire ou définitif, par l'État ou les collectivités territoriales.

Les entreprises publiques économiques (EPE) ne sont, quant à elles, pas soumises au DMP. Elles sont donc tenues d'élaborer et de faire adopter, par leurs organes sociaux, leurs propres procédures de passation de marchés, qui doivent être fondées sur les principes du DMP. C'est le cas également des établissements publics soumis à la législation régissant les activités commerciales, tels que les Établissement public à caractère industriel et commercial (EPIC), lorsqu'ils réalisent une opération qui n'est pas financée, totalement ou partiellement, sur concours temporaire ou définitif, par l'État (art. 8 et 9 du DMP).

En Algérie, il n'existe pas de liste exhaustive des EPE ; les entreprises publiques revêtent diverses formes juridiques, elles sont présentes dans tous les secteurs de l'économie et représentent plus de la moitié de l'économie formelle. Des entreprises telle que la Société nationale pour la recherche, la production, le transport, la transformation et la commercialisation des hydrocarbures (Sonatrach) et la Société nationale de l'électricité et du gaz (Sonelgaz) entrent dans la catégorie des EPE (U.S. Department of State, 2018[7]). Lors des discussions menées pendant les missions exploratoires, le ministère des Finances a évoqué une collaboration avec la Sonatrach dans le développement de leur cadre réglementaire, ce qui est considéré comme une bonne pratique. Étant donné le poids des entreprises publiques, le gouvernement algérien devrait mener une étude afin de lister toutes les EPE et s'assurer de la nécessité de développer leurs propres cadres réglementaires pour toutes les catégories d'achat.

D'autre part, le gouvernement algérien devrait encourager les EPE à consulter les autorités en charge des marchés publics afin de s'assurer que leur cadre réglementaire respecte les principes des marchés publics, tout en leur permettant de mener leurs activités efficacement.

\section{Publier les avis juridiques relatifs aux marchés publics}

Les cadres réglementaires régissant les marchés publics nécessitent la mise en place de mécanismes afin que les entités publiques, mais aussi les opérateurs économiques, puissent demander des clarifications et des conseils en matière de marchés publics (OCDE, 2016[3] $)$. Afin d'assurer une compréhension commune de certaines dispositions, plusieurs pays de l'OCDE ont créé un espace où sont publiés des avis portant sur des questions d'interprétation générale du cadre réglementaire. L'encadré 1.3 présente la rubrique des questions/réponses sur les marchés publics de la Direction des affaires juridiques du ministère français des Finances. 


\section{Encadré 1.3. Rubrique « questions/réponses » de la Direction des affaires juridiques- France}

En France, il est possible de saisir la Direction des affaires juridiques du ministère des Finances pour obtenir une consultation juridique sur une question relative aux marchés publics. Le bureau du conseil aux acheteurs de la Direction des affaires juridiques apporte une expertise en droit de la commande publique aux administrations centrales de l'État, à ses établissements publics, au service des achats de l'État et à l'ensemble des acheteurs publics. II diffuse l'information relative à la commande publique par la rédaction de « questions-réponses » publiées sur le site de la Direction des affaires juridiques. Ces consultations juridiques portent sur quatre grands thèmes :

- champ d'application,

- préparation de la procédure,

- mise en œuvre de la procédure,

- exécution des marchés.

Source : (Direction des affaires juridiques, ministère des Finances, France, 2018[8]).

En Algérie, l'ARMP a pour attribution « d'émettre des avis destinés aux services contractants, organes de contrôle, commissions des marchés, comités de règlement amiable des litiges et aux opérateurs économiques » (art. 213 du DMP). Cependant, l'ARMP n'a toujours pas été mise en place et, suivant une circulaire du ministère des Finances, ce rôle est partiellement assuré par la Direction du contrôle de la régularité des marchés publics de la Division des marchés publics au sein du ministère des Finances.

D'après les missions exploratoires de l'OCDE, la Division des marchés publics reçoit un nombre important de demandes de consultations qui peuvent porter sur des sujets similaires. Cependant des cas ont été identifiés dans le passé où pour une question similaire, des avis différents ont été émis. Pour limiter ce risque, la Division des marchés publics a décidé de créer récemment une bibliothèque interne des avis juridiques qu'elle émet afin de faciliter le traitement des demandes et d'assurer la cohérence des avis juridiques. Afin d'améliorer l'efficience du système et d'assurer la cohérence des avis juridiques qu'elle émet, la Division des marchés publics gagnerait à créer une rubrique sur son site web synthétisant ses avis sous forme de « questions-réponses».

\section{Renforcer la coordination du cadre réglementaire de la délégation de service public et des marchés publics}

Les partenariats publics privés (PPP), dont la délégation de service public est l'une des formes, sont des instruments complexes qui exigent des capacités avancées de la part des pouvoirs publics. Leur élaboration implique la création d'un système institutionnel robuste afin d'évaluer leur valeur ajoutée par rapport à la passation de marchés publics. Ce cadre institutionnel doit assurer que les autorités délégantes sont capables de classifier, mesurer et allouer le risque économique à la partie (autorité délégante ou délégataire) la mieux à même de le supporter, tout en protégeant l'intérêt public et en mettant en œuvre un contrôle budgétaire et comptable adapté (OCDE, 2011 $[9]$ ).

La Recommandation du Conseil de l'OCDE sur les marchés publics appelle les pays adhérents à harmoniser les principes applicables à la passation de marchés publics pour l'ensemble des services publics, s'il y a lieu, y compris pour les travaux publics, les PPP et les concessions. Lorsqu'ils fournissent des services publics dans le cadre d'un large éventail de dispositifs de partenariat avec le secteur privé, les Adhérents devraient assurer la plus grande cohérence possible entre les cadres et les institutions régissant la prestation des services publics (OCDE, 2015 [2]). 
Les délégations de service public sont une forme de PPP. Dans la réglementation algérienne, elles sont régies par les articles 207 à 210 du DMP. Les conventions de délégation de service public doivent respecter, pour leur passation, les principes des marchés publics (art. 209 du DMP), à savoir la liberté d'accès à la commande publique, l'égalité de traitement des candidats et la transparence des procédures. Le décret exécutif $n^{\circ}$ 18-199 du 2 août 2018 est venu compléter le DMP en matière de délégation des services publics des collectivités locales. Il détaille les règles applicables aux types de délégations de service public (concession, affermage, régie intéressée, gérance). Le cadre réglementaire des délégations de service public comprend également différentes réglementations sectorielles, telles que la loi $\mathrm{n}^{\circ} 2005$ 12 du 4 août 2005 relative à l'eau.

Le décret exécutif $n^{\circ}$ 18-199 du 2 août 2018 est le premier acte réglementaire spécialement dédié aux délégations de service public en Algérie. À ce titre, son adoption, en application des articles 207 à 210 du DMP, contribue à la cohérence du cadre réglementaire entre la passation des conventions de délégation de service public, d'une part, et les règles régissant les marchés publics, d'autre part. Cependant, le décret exécutif $n^{\circ} 18-199$ du 2 août 2018 ne mentionne pas l'ARMP alors que ses attributions en matière de délégations de services publics sont clairement mentionnées aux articles 213 et 214 du DMP. Ces attributions incluent notamment le suivi de la mise en œuvre de la réglementation et la conduite d'audits des procédures de passation des délégations de service public (art. 213 et 214). Afin d'assurer une meilleure cohérence entre les cadres réglementaires des marchés publics et des délégations de service public, l'Algérie devrait clarifier le rôle institutionnel de l'ARMP en matière de délégation de services publics en harmonisant les dispositions pertinentes entre les deux cadres réglementaires.

\subsubsection{La mise en place effective des fonctions centrales en matières de marchés publics}

Le bon fonctionnement d'un système de passation des marchés publics requiert la mise en place d'institutions efficaces remplissant différentes fonctions décrites dans le cadre réglementaire (graphique 1.1). Les marchés publics représentent une part importante du PIB, la mise en place de telles institutions relève donc souvent des priorités politiques des gouvernements. Selon les pays, les fonctions principales nécessaires au bon fonctionnement du système peuvent être exercées par une ou plusieurs institutions publiques. (OCDE, 2013 $\left.{ }_{[10]}\right)$

\section{Graphique 1.1. Les fonctions principales du cadre institutionnel du système de passation des marchés publics}

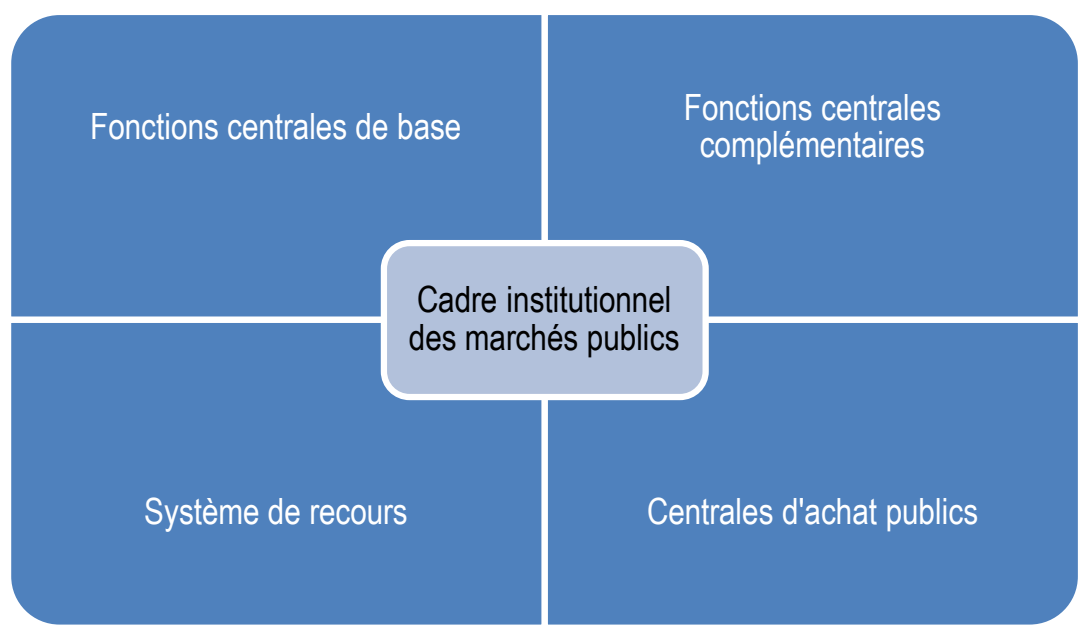

Source : (OCDE, 2013[10]). 
Généralement désignée par les gouvernements, l'institution en charge des fonctions centrales de base a notamment pour fonction le développement du cadre réglementaire en matière de marchés publics - y compris le développement et la mise en place des politiques et lignes directrices, ainsi que l'élaboration de documentation standard (documents d'appels d'offres, conditions générales pour les contrats publics, etc.).

Les fonctions centrales complémentaires visent à aider et à accompagner les entités publiques et les opérateurs économiques dans leurs activités relatives aux marchés publics. Elles comprennent notamment : le conseil et le support opérationnel, aussi bien à destination des entités publiques que des fournisseurs ; le contrôle du niveau de transparence du système ; le développement des politiques de professionnalisation et le renforcement des capacités dans le domaine des marchés publics. Ces fonctions sont particulièrement nécessaires lors de la mise en œuvre des réformes. Le cadre institutionnel des marchés publics comprend également les systèmes de recours permettant de contester les décisions d'attribution des marchés (voir chapitre 2) et, éventuellement, les centrales d'achats (voir chapitre 4). Par ailleurs, l'institution en charge de la réglementation doit jouir d'une certaine autorité au sein de l'administration, et notamment d'une certaine indépendance afin de pouvoir s'acquitter de ses responsabilités sans ingérence (OCDE, 2006[11]).

En Algérie, d'après le DMP, les fonctions centrales de base et complémentaires sont assurées par l'ARMP, qui est instituée auprès du ministère des Finances et dotée de l'autonomie de gestion. L'ARMP comprend un observatoire de la commande publique et un organe national de règlement des litiges (art. 213 et 214 du DMP). Les fonctions principales de l'ARMP sont décrites dans l'encadré 1.4.

\section{Encadré 1.4. Les fonctions principales de l'ARMP et des délégations de service public}

- Élaborer et suivre la mise en œuvre de la réglementation des marchés publics et des délégations de service public. L'ARMP émet, à ce titre, des avis destinés aux services contractants, organes de contrôle, commissions des marchés, comités de règlement amiable des litiges et aux opérateurs économiques.

- Informer, diffuser et vulgariser tous documents et informations relatifs aux marchés publics et aux délégations de service public.

- Initier les programmes de formation et promouvoir la formation dans le domaine des marchés publics et des délégations de service public.

- Effectuer annuellement un recensement économique de la commande publique.

- Analyser les données relatives aux aspects économiques et techniques de la commande publique et faire des recommandations au gouvernement.

- Constituer un lieu de concertation dans le cadre de l'observatoire de la commande publique.

- Auditer ou faire auditer les procédures de passation des marchés publics et des délégations de service public et leur exécution, à la demande de toute autorité compétente.

- Statuer sur les litiges nés de l'exécution des marchés publics conclus avec des partenaires cocontractants étrangers.

- Gérer et exploiter le système d'information des marchés publics.

- Entretenir des relations de coopération avec les institutions étrangères et les institutions internationales intervenant dans le domaine des marchés publics et des délégations de service public.

- Élaborer un code d'éthique et de déontologie des agents publics intervenant dans le contrôle, la passation et l'exécution des marchés publics et des délégations de service public.

- Tenir la liste des opérateurs économiques interdits de participer aux marchés publics.

Source : DMP. 
Malgré l'entrée en vigueur du DMP en 2015, l'ARMP n'a toujours pas été mise en place, ce qui nuit au bon fonctionnement du système de passation des marchés publics. En effet, l'ARMP ne peut exercer ses missions avant la promulgation du décret exécutif sur son organisation et ses modalités de fonctionnement. L'absence de mise en place effective de l'ARMP empêche l'élaboration d'une doctrine partagée sur l'application du droit et provoque un manque de clarté du système de passation des marchés publics dans son ensemble. Cette situation est, de plus, préjudiciable au développement du système de passation des marchés.

Dans l'attente de la mise en place de l'ARMP (ou de toute autre instance en charge des fonctions de base centrales et complémentaire), la Division des marchés publics du ministère des Finances est chargée du système de passation des marchés publics. Cependant, cette division ne peut assurer qu'une partie des missions dévolues à l'ARMP et n'a pas l'autonomie de gestion et l'indépendance requises pour assurer les fonctions centrales conformément aux bonnes pratiques internationales. Par ailleurs, actuellement, elle ne remplit pas toutes les fonctions prévues pour l'ARMP. En outre, les attributions de cette division - telles que mentionnées dans le décret exécutif $n^{\circ}$ 07-364 du 28 novembre 2007 portant organisation de l'administration centrale du ministère des Finances - ne sont pas cohérentes avec le cadre réglementaire actuel. En effet, la Division des marchés publics regroupe une direction du contrôle de la régularité des marchés publics et une direction des marchés publics. L'une des tâches historiques de cette dernière est d'assurer le secrétariat permanent de la commission nationale des marchés, alors même que les évolutions réglementaires du DMP ne prévoient plus de commission nationale des marchés.

Il est donc nécessaire pour le gouvernement algérien de mettre en place l'ARMP (ou une autre entité chargée des fonctions de base centrales et complémentaires) dans les plus brefs délais, pour assurer le bon fonctionnement du système et la cohérence de son cadre réglementaire avec son cadre institutionnel. Par ailleurs, en attendant la promulgation et l'entrée en vigueur du décret exécutif relatif à la mise en place de l'ARMP (ou une autre entité), le ministre des Finances gagnerait à demander à la Division des marchés publics de prendre en charge certaines attributions de l'ARMP afin de mieux préparer sa mise en place effective. Cette décision nécessitera des aménagements réglementaires.

\subsubsection{Instaurer un dialogue avec l'ensemble des parties prenantes du système de passation des marchés publics}

La participation des parties prenantes dans l'évolution du système de passation des marchés publics présente de nombreux avantages. Elle permet d'identifier les effets non désirables ou néfastes des nouvelles mesures, et de mieux évaluer leur efficacité grâce aux retours des différents acteurs. Ces parties prenantes comprennent les entités publiques (ministères centraux, collectivités locales), mais aussi les opérateurs économiques et leurs représentants (chambres de commerce, associations sectorielles), et enfin des associations ou autres représentants de la société civile. L'implication des parties prenantes dans les réformes du système de passation des marchés publics permet de renforcer leur confiance dans ledit système. C'est un enjeu important concernant les opérateurs économiques, dont la confiance dans le système est un déterminant clé de leur participation aux procédures de mise en concurrence.

L'implication des parties prenantes est conforme aux exigences de transparence du cadre institutionnel et réglementaire des marchés publics, détaillées dans le chapitre 3 du présent rapport (OCDE, 2018[12]). En effet, la Recommandation du Conseil de l'OCDE sur les marchés publics stipule que les adhérents doivent " concevoir et suivre une procédure normalisée pour modifier le système et les politiques de passation de marchés publics. " Cette procédure normalisée devrait garantir « des consultations publiques et la formulation de commentaires par le secteur privé et la société civile, assurer la publication des conclusions de la phase de consultations et expliquer les options retenues, le tout de façon transparente ». (OCDE, 2015[2]) 


\section{Une approche inclusive pour toute réforme dans le domaine des marchés publics}

De nombreux pays de l'OCDE ont mis en place des mécanismes institutionnels permettant aux parties prenantes de soumettre des commentaires sur les projets de lois et de réglementations en matière de marchés publics, ou de fournir des retours et des propositions sur les systèmes de passation des marchés (encadré 1.5). Ces mécanismes prennent plusieurs formes :

- la consultation en ligne des parties prenantes concernant les projets de lois ou de réglementation dans le domaine des marchés publics,

- la consultation par le biais de questionnaires ciblés, par exemple auprès des opérateurs économiques et des organisations de la société civile,

- la plateforme en ligne permettant des dialogues et des commentaires croisés entre parties prenantes quant à l'évolution du système des marchés publics,

- les tables rondes ou les groupes de travail inclusifs permettant d'impliquer un large éventail de parties prenantes (opérateurs économiques, société civile, experts académiques, etc.) dans l'évolution du système des marchés publics.

\section{Encadré 1.5. La consultation des parties prenantes dans les pays de l'OCDE}

Dans les pays de l'OCDE, la consultation publique en ligne est la forme la plus courante de collecte des commentaires des parties prenantes, elle concerne en effet 33 des 34 pays de l'OCDE. Dans une grande majorité des pays (28 sur 34 ), les commentaires recueillis ou leur synthèse sont également mis en ligne. Ces commentaires sont pris en compte lors des analyses d'impact de la réglementation ou lors d'autres phases du processus décisionnel.

Aux États-Unis, le Conseil national des responsables des achats fédéraux (Chief Acquisition Officers Council) et la Direction de la politique fédérale en matière de marchés publics (Office of Federal Procurement Policy) ont lancé une plateforme en ligne en 2014 pour permettre aux parties prenantes d'identifier les problèmes, les barrières à l'accès aux marchés publics et de proposer des solutions. II s'agit de recueillir des idées d'améliorations du système fédéral des marchés publics afin, le cas échéant, de réviser le cadre réglementaire (révisions des réglementations ou de la législation en vigueur).

Au-delà de la simple consultation en ligne des parties prenantes, certains pays de l'OCDE conduisent des consultations actives des parties prenantes, par le biais de tables rondes ou de groupes de travail. Par exemple, en Irlande, l'Agence des marchés publics (Office of Government Procurement) consulte directement les associations représentatives des PME afin de prendre en compte leurs points de vue sur l'évolution du système de passation des marchés publics et d'identifier d'éventuels obstacles à la participation des PME. En Belgique, la Commission des marchés publics réunit l'ensemble des parties prenantes (services contractants, corps d'inspection et de contrôle, associations représentatives du secteur privé, syndicats). Tous les avant-projets de lois ou d'arrêtés royaux relatifs aux marchés publics lui sont soumis pour avis. Au Mexique, avec le soutien de l'OCDE, le ministère de l'Administration publique a mis en place un groupe de travail inclusif pour concevoir l'évolution du portail électronique des marchés publics.

Sources : (OCDE, 2018[13] ; OCDE, 2016[14] $;$ OCDE, 2016[15]). 
En Algérie, le décret présidentiel portant réglementation des marchés publics (dont le DMP de 2015 constitue la dernière version), qui constitue le pilier de la réglementation en matière de marchés publics, a fait l'objet de nombreuses révisions. Il a en effet été révisé ou amendé sept fois depuis 2002. Comme ont pu le constater les missions exploratoires de l'OCDE, certains ministères (en-dehors du ministère des Finances) et les parties prenantes, en particulier les opérateurs économiques et la société civile, n'ont pas réellement eu l'occasion de participer à ces révisions.

Comme détaillé dans la section 4.4 en l'absence de données fiables et actualisées sur la passation des marchés publics, l'évaluation du système de passation des marchés publics dont dispose le ministère des Finances est limitée. L'implication des parties prenantes (en particulier des services contractants de plusieurs ministères et des opérateurs économiques) dans la formulation des réformes du cadre réglementaire des marchés publics est donc d'autant plus importante. Afin de permettre aux différentes parties prenantes de prendre part au diagnostic et à la formulation des évolutions du système des marchés publics, l'Algérie gagnerait à mettre en place une consultation systématique des parties prenantes avant toute modification du cadre réglementaire des marchés publics, qu'il s'agisse du DMP ou même des principaux actes réglementaires d'application (arrêtés, décrets exécutifs...).

Par ailleurs, un compte-rendu ou un résumé des retours sur le projet d'acte réglementaire devrait être publié en ligne suite à ces consultations publiques. De plus, au vu de l'importance des marchés publics en Algérie et du niveau relativement faible de numérisation des pratiques, ce processus de consultation publique en ligne pourrait être enrichi par des rencontres avec les parties prenantes.

\section{La méthode $R A C l$, outil de planification de réforme des marchés publics}

Le présent rapport comprend un grand nombre de recommandations en vue de réformer le système de passation des marchés publics en Algérie. L'entité en charge des fonctions centrales de base et complémentaires en matière de marchés publics est la mieux placée pour coordonner la mise en œuvre de ces différentes recommandations. Elle aura également à piloter des évolutions fondamentales pour le système de passation des marchés publics, telles que le lancement du portail électronique des marchés publics. Ces missions nécessitent l'implication d'un grand nombre d'entités publiques et de parties prenantes. La méthode RACl, outil de gestion de projets permettant de clarifier et de communiquer les rôles et les responsabilités des différents intervenants au sein de chaque processus et activité, pourrait être utile (encadré 1.6).

\section{Encadré 1.6. Gestion de projets complexes : la méthode RACI}

La méthode $\mathrm{RACl}$ est utile dans la gestion de projets complexes qui comprennent un grand nombre de parties prenantes, afin de clarifier les tâches de chacun et d'identifier les doublons, duplications et autres inefficacités. II s'agit d'une matrice de responsabilités permettant de définir :

- $\mathrm{R}=$ Responsible ou Réalisateur : les entités ou individus qui réalisent le projet.

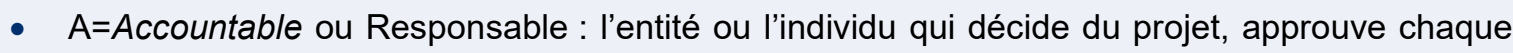
étape et porte la responsabilité du projet.

- $\quad \mathrm{C}=$ Consultés : entités ou individus qui doivent être consultés et donnent leur avis sur les sujets sur lesquels ils sont experts.

- I= Informés : entités ou individus qui doivent être informés suite aux décisions ou aux étapes du projet.

Source : (Santos, 2018[16]). 
En partenariat avec la Commission européenne, l'OCDE a conduit un projet de soutien à la réforme des marchés publics à Malte dont l'axe principal était la modernisation de la Direction générale des contrats du ministère des Finances. À cette occasion, le gouvernement de Malte a utilisé la méthode RACI pour identifier le rôle de chaque entité publique dans le processus de réforme. Le tableau 1.2 présente un extrait de l'assignation des responsabilités aux différentes entités publiques.

\section{Tableau 1.2. Utilisation de la méthode RACl pour la réforme des marchés publics à Malte}

\begin{tabular}{|c|c|c|c|c|c|c|c|}
\hline Action & $\begin{array}{l}\text { Bureau du } \\
\text { Premier } \\
\text { ministre }\end{array}$ & $\begin{array}{l}\text { Comité de } \\
\text { suivi }\end{array}$ & $\begin{array}{l}\text { Équipe-projet } \\
\text { de mise en } \\
\text { œuvre }\end{array}$ & $\begin{array}{l}\text { DC Unité } \\
\text { Réglementation }\end{array}$ & $\begin{array}{l}\text { DC Unité } \\
\text { Stratégie }\end{array}$ & $\begin{array}{c}\text { DC } \\
\text { Contrôle }\end{array}$ & $\begin{array}{l}\text { DC } \\
\text { Équipe } \\
\text { TIC }\end{array}$ \\
\hline $\begin{array}{l}\text { Développement du plan stratégique de la réforme des } \\
\text { marchés publics à Malte }\end{array}$ & A & $\mathrm{R}$ & C & $\mathrm{C}$ & C & C & C \\
\hline $\begin{array}{l}\text { Mise en place de lignes directrices sur les procédures } \\
\text { de marchés publics }\end{array}$ & C & $A$ & C & $\mathrm{R}$ & C & C & 1 \\
\hline $\begin{array}{l}\text { Définir les responsabilités des services contractants, } \\
\text { des unités d'achat ministérielles et du DC pour } \\
\text { s'assurer du respect des procédures }\end{array}$ & C & A & C & $\mathrm{R}$ & C & C & 1 \\
\hline $\begin{array}{l}\text { Définir les procédures pour planifier les appels d'offres } \\
\text { (y compris les ressources nécessaires) }\end{array}$ & C & A & C & C & $\mathrm{R}$ & C & C \\
\hline Définir le système d'assurance de la qualité & 1 & A & C & C & C & $\mathrm{R}$ & C \\
\hline $\begin{array}{l}\text { Préparer des lignes directrices sur la mise en place } \\
\text { des accords-cadres }\end{array}$ & C & A & - & $\mathrm{R}$ & C & C & C \\
\hline $\begin{array}{l}\text { Mettre en place une Unité de centralisation des } \\
\text { besoins pour les accords-cadres }\end{array}$ & A & $\mathrm{R}$ & C & - & - & - & - \\
\hline
\end{tabular}

Note: DC signifie Direction générale des contrats du ministère des Finances. Afin de faciliter la lecture, les entités Responsables ( $A=A c c o u n t a b l e)$ sont marquées en bleu, tandis que les entités Réalisatrices ( $R=$ Responsible) sont mises en évidence en rouge. Les autres parties prenantes sont consultées ( $\mathrm{C}=$ Consultés) à chaque étape de la conduite du changement, ou informées ( $\mathrm{l}=$ Informés) autant que nécessaire.

Source :Adapté de (OCDE, 2019[17]).

Le ministère des Finances et l'entité en charge des fonctions centrales de base et complémentaires en matière de marchés publics pourraient utiliser la méthode $\mathrm{RACl}$ pour assigner clairement les responsabilités des différents acteurs institutionnels et piloter les stratégies et réformes du système de passation des marchés publics en Algérie (mise en œuvre des recommandations du présent rapport, lancement du portail électronique des marchés publics, stratégie pour l'utilisation des marchés publics comme un levier stratégique pour atteindre des objectifs de politiques, etc.).

\subsection{Promouvoir les procédures concurrentielles et limiter le recours aux exceptions}

Les procédures concurrentielles devraient constituer la norme pour la passation des marchés publics. En effet, elles permettent non seulement d'obtenir des prix avantageux ou un meilleur rapport qualité-prix pour l'organisme public, mais aussi d'assurer l'intégrité des conditions de passation du marché et d'éviter toute influence illégitime dans le choix de l'attributaire (OCDE, 2015[2]). Les études empiriques qui ont quantifié les avantages d'une plus grande concurrence dans les marchés publics concluent qu'une réglementation et des procédures plus transparentes et favorables à la concurrence ont un impact significatif et permettent d'obtenir des prix plus compétitifs (Estache et limi A., 2008[18]). En outre, la concurrence sur les marchés publics a d'autres avantages, car elle génère des externalités positives pour l'ensemble de l'économie (voir graphique 1.2). 
Graphique 1.2. Avantages de la concurrence sur les marchés publics

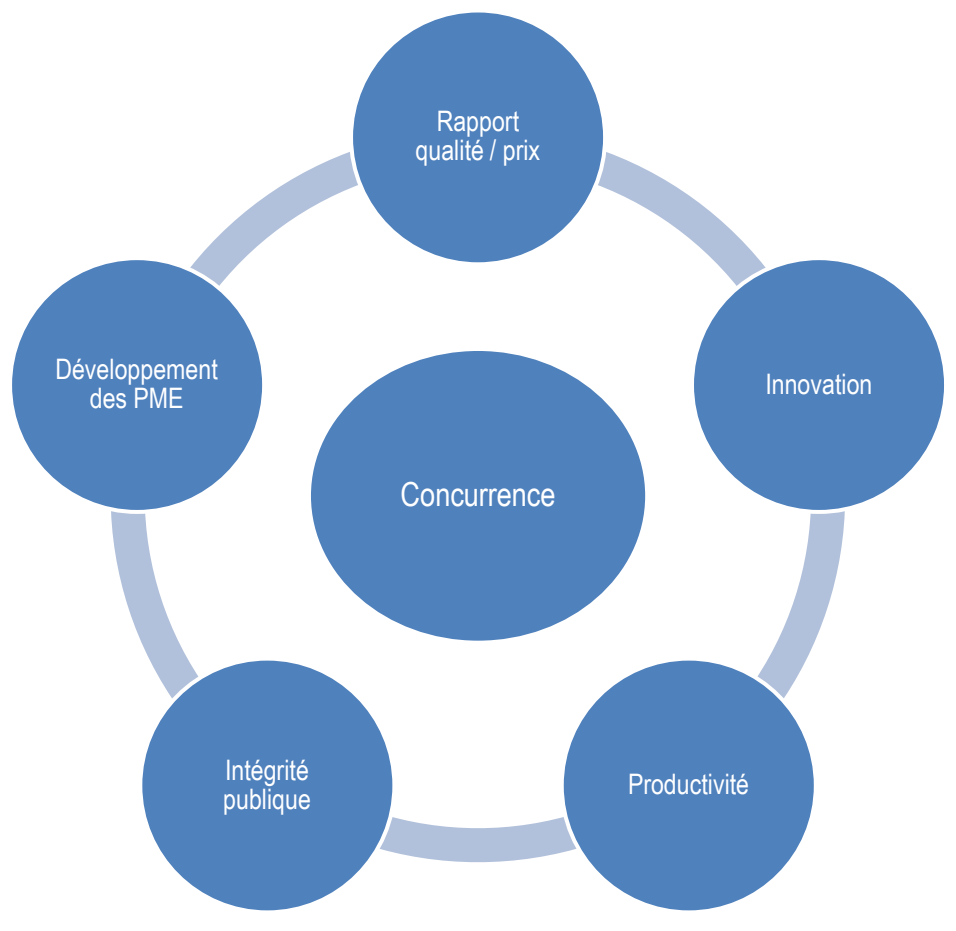

Source : (OCDE, à paraître $[5])$.

La concurrence dans les marchés publics accroît le niveau général de concurrence sur l'ensemble des marchés des biens et services et contribue à un environnement économique qui incite à l'innovation et à la diffusion des nouvelles technologies. Au final, ces externalités positives favorisent les gains de productivité $\left(\mathrm{OCDE}\right.$, à paraître $\left[{ }_{[5]}\right)$. De tels gains sont également favorables, à long terme, à la compétitivité des entreprises domestiques sur les marchés internationaux, avec des effets bénéfiques sur la balance commerciale (Sakakibara et Porter, 2001[19]).

Par ailleurs, les pouvoirs adjudicateurs ont intérêt à améliorer la participation des PME aux marchés publics pour accroître la concurrence. Les pouvoirs adjudicateurs peuvent en effet bénéficier de l'apport de nouveaux entrants innovants, qui sont généralement des PME, pour stimuler la concurrence, notamment en remettant en cause les solutions des opérateurs économiques établis (OCDE, 2017[20]). Mais les exceptions aux procédures concurrentielles sur les marchés publics sont une barrière à l'accès des PME (voir chapitre 5).

Malgré les avantages de la concurrence sur les marchés publics et le principe d'égalité d'accès à la commande publique, les entités publiques font parfois face à des circonstances exceptionnelles qui justifient le recours à des procédures moins concurrentielles (OCDE, à paraître $\left.{ }_{[5]}\right)$. Cette hiérarchie des procédures (de la plus concurrentielle à la moins concurrentielle), ainsi que les risques associés à chaque option sont exposés dans le graphique 1.3. 


\section{Graphique 1.3. Hiérarchie des procédures de passation des marchés publics}

De la procédure la plus concurrentielle (appel d'offres) à l'attribution directe (gré à gré)

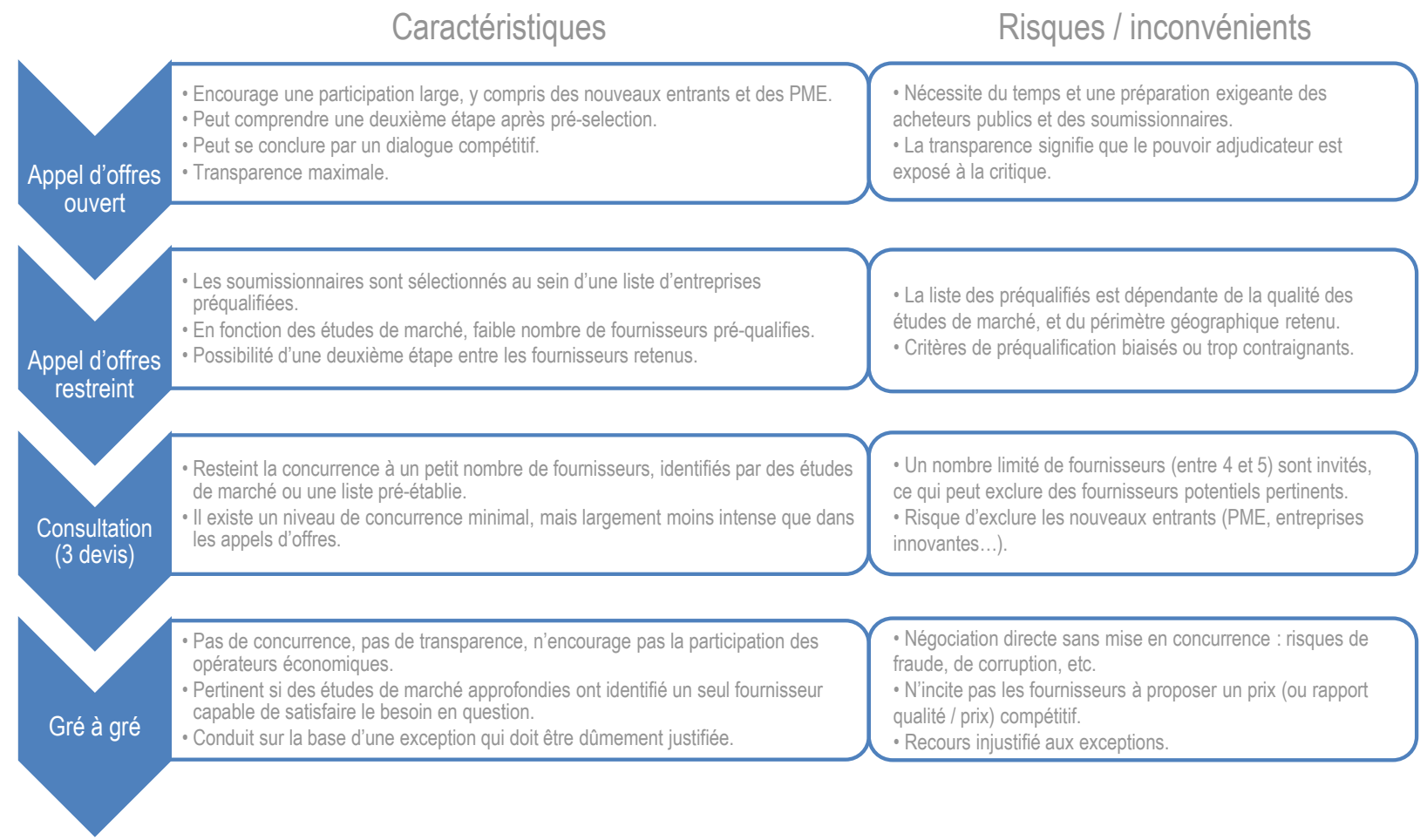

Source : (OCDE, à paraître[5]).

En Algérie, le cadre réglementaire régissant les marchés publics prévoit plusieurs procédures avec des niveaux concurrentiels différents décrits dans le graphique 1.4. Conformément aux bonnes pratiques internationales, l'appel d'offres ouvert est la procédure d'achat par défaut. D'après les statistiques compilées par l'équipe de l'OCDE sur la base d'une sélection d'avis du Bulletin officiel des marchés de l'opérateur public (Bomop) sur la semaine du 18 au 24 novembre 2018, les appels d'offres ouverts représentent l'essentiel des avis d'appel d'offres (93.5\%), le reste étant constitué d'appels d'offres restreints $(6.5 \%)$. En l'absence de données officielles fournies par l'Algérie ou disponibles sur le Bomop, cette analyse a permis de produire des statistiques portant sur une quarantaine d'avis d'appel d'offres, ce qui offre une bonne première approximation. Cependant, les données disponibles ne permettent pas d'estimer les montants respectifs des appels d'offres ouverts ou restreints. Par ailleurs, aucun chiffre n'est disponible sur les procédures non formalisées, qui représentent une part importante des achats publics (gré à gré simple ou après consultation). 
Graphique 1.4. Les différentes procédures de passation des marchés publics en Algérie

\section{Marché de travaux ou de fournitures}

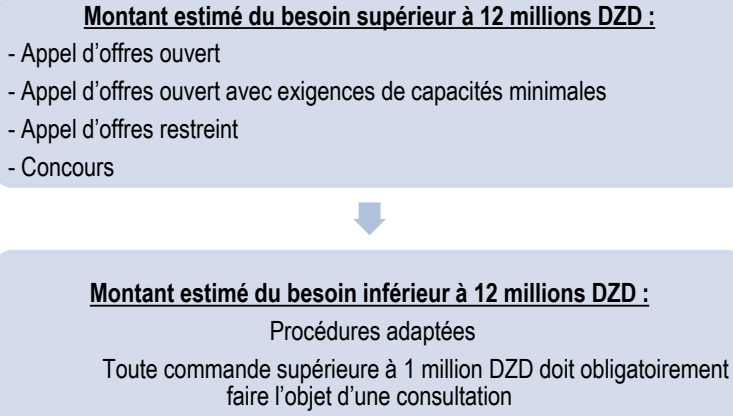

Marché d'études ou de services

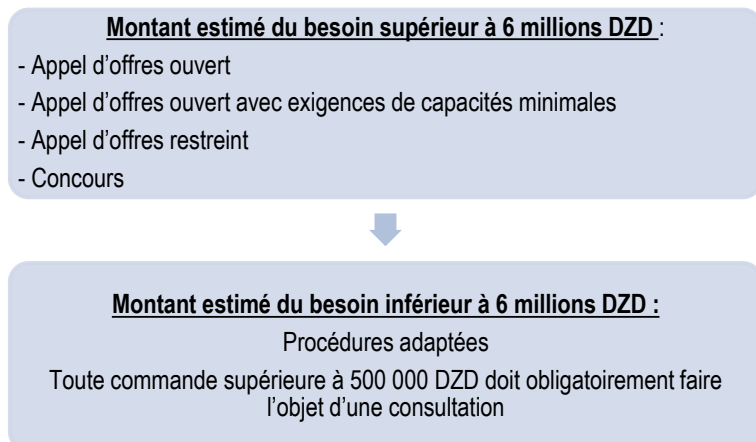

Source : DMP.

\subsubsection{Harmoniser les règles d'attribution en dessous des seuils}

\section{Ajuster les seuils d'appels d'offres aux catégories d'achat}

Dans certains pays, les seuils de procédure formalisée distinguent les marchés de fournitures, de services et des travaux publics. Ce seuil peut être plus élevé pour les travaux publics. Par exemple, en Tunisie, le seuil des procédures formalisées pour les travaux publics est de 500000 TND (dinars tunisiens, soit environ 147000 EUR [euros]), supérieur au seuil pour les fournitures de biens et services (300 000 TND, soit environ 88000 EUR) (JORT, 2014[21])).

En Algérie, il existe deux seuils de procédures formalisées : un seuil pour les marchés de fourniture et de travaux fixé à 12 millions DZD (environ 88500 EUR), et un autre pour les études et les services fixé à 6 millions DZD (dinars algériens, environ 44250 EUR, voir DMP art. 13). Par ailleurs, l'article 21 du DMP prévoit un second seuil en dessous duquel les services contractants peuvent recourir au gré à gré simple (pas de consultation obligatoire du marché) pour leurs acquisitions : 1 million DZD pour les fournitures et les travaux (environ 7400 EUR) et 500000 DZD (environ 37000 EUR) pour les études et les services.

Le seuil concernant les fournitures est donc le même que celui portant sur les travaux de construction. Les seuils de procédure formalisée doivent être déterminés avec soin, en prenant en compte plusieurs critères, y compris : les coûts administratifs de l'appel d'offres, le montant moyen constaté des contrats, et le niveau macro de concurrence sur le marché économique. En effet, un seuil trop faible pour les travaux peut entraîner certains inconvénients liés à un formalisme disproportionné pour les travaux de maintenance, d'entretien ou de réparation courants, par exemple. Plus particulièrement, les coûts administratifs des procédures doivent être justifiés par les bénéfices de la procédure d'appel d'offres en termes de transparence et de mise en concurrence des opérateurs économiques (OCDE, à paraître $[5])$. L'Algérie pourrait donc s'assurer que les seuils de passation des procédures formalisées permettent vraiment d'atteindre un niveau de concurrence adéquat tout en prenant en compte les coûts associés à ce formalisme. 


\section{Harmoniser et préciser les règles d'attribution en dessous des seuils}

Les cadres réglementaires régissant les marchés publics prévoient le plus souvent des procédures simplifiées en dessous des seuils d'appel d'offres, tout en respectant les grands principes régissant les marchés publics. Ces procédures simplifiées déterminent généralement des règles de mise en concurrence et de publicité allégées (OCDE, 2011[22]). En tout état de cause, ces procédures doivent respecter les conditions décrites dans l'encadré 1.7 .

\section{Encadré 1.7. Les conditions que doivent respecter les procédures simplifiées}

Les procédures de passation des marchés publics pour les contrats en dessous des seuils devraient :

- Être efficaces et performantes, et par conséquent utiliser des processus, échéanciers et documents reflétant les besoins et les résultats attendus de ces marchés.

- Être proportionnées à l'objet du contrat et éviter une bureaucratie inutile afin de ne pas imposer une charge administrative trop complexe ou importante, ou des coûts disproportionnés à l'entité contractante ou à l'opérateur économique.

- Assurer une concurrence équitable de façon à attirer plus de soumissionnaires, y compris des PME et des startups qui pourraient être particulièrement attirées par les contrats à plus faible valeur.

- Être transparentes pour favoriser une concurrence équitable et prévenir le favoritisme et la corruption.

- Être conformes aux principes des marchés publics.

- Utiliser le plus efficacement possible l'argent public, assurant ainsi une allocation optimale des ressources pour parvenir aux résultats attendus.

Source : (OCDE, 2011[22]).

Dans les pays de l'UE, les contrats en dessous des seuils européens sont généralement sujets à des procédures simplifiées (ou procédures adaptées) régies dans chaque pays par des règles moins strictes que celles qui président aux procédures formalisées. Comme décrit au tableau 1.3, les seuils sont prévus pour différentes catégories d'entités, et une distinction est faite entre les marchés de travaux et les autres. Par ailleurs, les procédures sans publicité ni mise en concurrence sont souvent autorisées pour les contrats publics portant sur de très faibles montants (1 000 à 33000 EUR selon les pays) afin que les procédures de passation des marchés soient adaptées à l'objet et à la valeur estimée du contrat (OCDE, 2011 [22]) (OCDE, 2018[23]). 
Tableau 1.3. Seuils des procédures formalisées dans I'UE

\begin{tabular}{l|l|c}
\hline \multicolumn{1}{c|}{ Type de contrat public } & \multicolumn{1}{c}{ Pouvoirs adjudicateurs } & Seuils \\
\hline $\begin{array}{l}\text { Fournitures, contrats de services et } \\
\text { concours (architecture) }\end{array}$ & Ministères et organes du gouvernement central & EUR144 000 \\
\hline $\begin{array}{l}\text { Fournitures, contrats de services et } \\
\text { concours (architecture) }\end{array}$ & Collectivités territoriales, secteur de la Défense nationale & EUR221 000 \\
\hline $\begin{array}{l}\text { Fournitures, contrats de services et } \\
\text { concours (architecture) }\end{array}$ & $\begin{array}{l}\text { Acheteur public qui exerce une activité d'opérateur de réseaux (production, } \\
\text { transport ou distribution d'électricité, gaz, eau, etc.) }\end{array}$ & EUR443000 \\
\hline \begin{tabular}{l} 
Contrat de travaux \\
\hline
\end{tabular} & Tout pouvoir adjudicateur & EUR 5548 000 \\
\hline
\end{tabular}

Note : Au-dessus de ces seuils, dans tous les pays de l'UE, les marchés publics sont passés conformément aux règles de procédure formalisée et les avis publiés sur la plateforme européenne TED. Les pays ont également des seuils nationaux en dessus desquels certaines formalités sont obligatoires (25000 EUR en France, 33000 EUR aux Pays-Bas, 30000 EUR en Pologne, etc...) : c'est la procédure adaptée. Seuils valables jusqu'au 1er janvier 2020.

Source : (Union Européenne, 2017[24])

Comme le cadre réglementaire des pays de l'OCDE, le DMP prévoit des exceptions au formalisme de l'appel d'offres : les procédures formalisées ne sont en effet pas obligatoires pour les marchés dont le montant estimé des besoins est inférieur à 12 millions DZD pour les travaux et les fournitures et 6 millions DZD pour les études ou les services (art.13 du DMP). En-dessous de ces seuils, chaque service contractant élabore des procédures internes pour la passation du marché. Le cadre réglementaire mentionne uniquement que les services contractants doivent procéder à la consultation par écrit des opérateurs économiques qualifiés et que les besoins doivent faire l'objet d'une publicité adéquate (art. 14 du DMP). Cependant, aucun arrêté du ministre des Finances ne complète ni ne détaille ces dispositions, alors même qu'il est prévu « en cas de besoin » à l'article 13 du DMP. De fait, les dispositions de l'article 13 nécessiteraient des précisions relatives, par exemple, au nombre de fournisseurs à consulter, aux règles de publicité, à la durée de validité des offres, etc.

Par ailleurs, les missions exploratoires de l'OCDE relèvent l'absence de manuel pour l'élaboration des procédures en dessous des seuils, lequel serait utile aux agents chargés de la passation des marchés publics. De nombreux pays ont développé de tels manuels. La France, par exemple, a développé des fiches techniques à destination des acheteurs publics pour les marchés en dessous des seuils fixés par le décret relatif aux marchés publics (Direction des affaires juridiques, France, 2016[25]). Sans lignes directrices claires, les procédures internes prévues par chaque entité publique peuvent être très différentes.

Afin d'assurer le respect des principes des marchés publics et d'améliorer l'efficacité et l'intégrité du système, mais aussi pour assurer une uniformité des règles appliquées aux différentes entités publiques, le ministre des Finances devrait publier un arrêté ou une circulaire apportant plus de précisions sur les procédures adaptées. Par ailleurs, pour assurer une compréhension commune de ces dispositions, le ministère des Finances - ou l'ARMP une fois mise en place - gagneraient à publier des procédures internes types, dont les entités publiques pourraient s'inspirer pour rédiger leurs propres procédures internes.

Par ailleurs, l'équipe de l'OCDE a effectué des recherches sur le Bomop et a constaté que les avis de consultation en procédure adaptée n'y sont pas publiés, ce qui remet en question le respect de la transparence des procédures. Bien que l'Algérie n'ait pas transmis de données relatives aux procédures en dessous des seuils, leurs montants représentent une part considérable des dépenses de l'État. Enfin, dans le cadre de la consultation des fournisseurs devant faire l'objet d'une publicité adéquate (art. 14 du DMP), les services contractants qui optent pour la publication d'un avis de consultation pourraient avoir l'obligation de le publier au Bomop et sur le futur portail des marchés publics, afin de garantir l'information du plus grand nombre de fournisseurs. 


\subsubsection{Revoir le système d'exclusion et d'exceptions aux appels d'offres ouverts}

Les pays où les systèmes de marchés publics sont plus transparents et où les exceptions aux appels d'offres ouverts sont strictement délimitées dans le cadre réglementaire et dûment justifiées, connaissent une participation plus active des opérateurs économiques aux marchés publics (Knack, Biletska et Kacker, 2017[26]).

En effet, les procédures concurrentielles devraient constituer la norme, afin que la passation de marchés publics soit au service des gains d'efficience, de la lutte contre la corruption et de la concurrence (OCDE, 2015[2]). Toutefois, certaines circonstances peuvent justifier le recours à des procédures moins concurrentielles que l'appel d'offres ouvert. Elles doivent être prévues dans les cadres réglementaires $\left(\mathrm{OCDE}\right.$, à paraître $\left.{ }_{[5]}\right)$. Par ailleurs, " au cas où des circonstances exceptionnelles justifieraient des limites à l'appel à la concurrence et la sollicitation d'une source unique, ces exceptions devraient être limitées, prédéfinies et dûment justifiées, et elles devraient faire l'objet d'une surveillance adaptée qui tiendrait compte du risque accru de corruption, y compris de la part de fournisseurs étrangers » (OCDE, 2015[2]). En plus des exceptions, la concurrence dans les marchés publics est également liée aux exclusions prévues dans le cadre réglementaire(voir définitions à l'encadré 1.2).

\section{Revoir et préciser le cadre réglementaire pour le recours à l'appel d'offres restreint}

L'appel d'offres restreint (ou son équivalent) existe dans de nombreux pays de l'OCDE. II s'agit d'une procédure de pré-qualification des candidats permettant de constituer une liste d'opérateurs économiques qualifiés qui seront ensuite admis à soumissionner. La pré-qualification doit cependant être conçue de façon à ne pas restreindre la concurrence de façon excessive et à encourager une large participation de soumissionnaires, y compris de nouveaux entrants (OCDE, 2015[2]). La Nouvelle-Zélande dispose d'une procédure d'appel d'offres en deux étapes (multi-step procurement), constituée d'un appel à manifestation d'intérêt suivi d'une phase de soumission ouverte aux candidats présélectionnés. La réglementation européenne offre également un exemple de procédure restreinte dans laquelle tout opérateur économique peut soumettre une demande de participation en réponse à un avis d'appel à la concurrence. Le service contractant décide ensuite des candidats admis à soumissionner, qui doivent être au minimum au nombre de cinq afin d'assurer un nombre de candidats suffisant pour garantir une concurrence réelle (Union Européenne, 2014[27]).

En Algérie, les services contractants ont également la possibilité de recourir à l'appel d'offres restreint, dans lequel seuls les candidats présélectionnés sont invités à soumissionner (art. 45 et 46 du DMP). L'appel d'offres restreint est possible (mais pas obligatoire) à l'occasion des marches d'études (par exemple, études d'ingénierie) ou d'opérations complexes et/ou d'importance particulière, voire l'acquisition de fournitures spécifiques, à caractère répétitif (art. 45 du DMP). Sa publication est obligatoire dans les mêmes conditions qu'un appel d'offres ouvert (art. 61 du DMP). D'après les statistiques compilées par l'équipe de l'OCDE sur la base d'une sélection d'avis du Bomop, l'appel d'offres restreint a représenté $6.5 \%$ des appels d'offres sur la semaine du 18 au 24 novembre 2018.

Les articles 45 et 46 régissant les appels d'offres restreints manquent de clarté et de précision, notamment concernant les modalités pour conduire un appel d'offres restreint. Les services peuvent recourir à une liste restreinte ou short list d'opérateurs économiques qualifiés, dressée antérieurement à l'appel d'offres, pour la présélection des soumissionnaires. Cette short list dressée «à l'occasion de la réalisation d'opérations d'études, d'ingénierie complexe ou d'importance particulière et/ou d'acquisition de fournitures spécifiques, à caractère répétitif » est renouvelée seulement tous les trois ans (art. 45 du DMP). Cet article suggère qu'il n'y a donc pas de publication d'avis d'appel à la concurrence, alors même qu'elle est obligatoire d'après l'article 61 du DMP. En outre, les modalités d'établissement de la shortlist d'opérateurs économiques qualifiés pour les appels d'offres restreints ne sont pas précisées. En effet, l'arrêté du ministre des Finances pourtant prévu « en tant que besoin » à l'article 45 du DMP n'a pas été publié, laissant une marge d'appréciation excessive aux services contractants en la matière. Son adoption est donc nécessaire pour préciser les modalités de mise en œuvre des appels d'offres restreints. 
Par ailleurs, la procédure d'appel d'offres restreint avec présélection des candidats sur la base d'une shortlist renouvelée tous les trois ans seulement à la discrétion des services contractants présente le risque d'une réduction excessive de la concurrence et de l'accès des opérateurs économiques, et notamment des nouveaux entrants, aux opportunités des marchés publics. Cela est d'autant plus vrai que, d'après le DMP, la liste des projets qui peuvent faire l'objet d'un appel d'offres restreint est fixée par décision du responsable de l'institution publique ou du ministre concerné, c'est-à-dire par le service contractant lui-même.

En conséquence, l'Algérie pourrait rendre obligatoire la publication d'un avis d'appel à la concurrence dans le cadre de tous les appels d'offres restreints, afin d'assurer une présélection équitable et transparente des opérateurs économiques admis à soumissionner. Afin de garantir un nombre suffisant de candidats, la réglementation algérienne pourrait également prévoir un nombre minimal de candidats invités à soumissionner pour tous les appels d'offres restreints.

\section{Encadrer le recours aux exceptions à l'appel d'offres}

Une exception dûment justifiée à la procédure d'appel d'offres implique que la justification du recours à l'exception doit être documentée et disponible en cas d'audit de la procédure de passation du marché. La clarté et la précision des règles régissant le recours aux exceptions sont importantes pour les services contractants comme pour les opérateurs économiques. En effet, un pouvoir discrétionnaire excessif des services contractants dans le recours aux exceptions à l'appel d'offres a un impact négatif sur la perception des fournisseurs, qui peuvent douter du caractère prévisible et équitable du choix d'une procédure nonconcurrentielle et donc ne pas participer aux marchés publics (Knack, Biletska et Kacker, 2017[26]) (Ware G. et al., 2007[28]).

En Algérie, conformément aux bonnes pratiques internationales, le cadre réglementaire prévoit une liste exhaustive des cas d'exceptions à l'appel d'offres. Ces exceptions peuvent prendre la forme du gré à gré simple (art. 49 et 50 du DMP) et du gré à gré après consultation (art. 51 et 52 du DMP). Le gré à gré après consultation est une procédure plus concurrentielle que le gré à gré simple car elle implique nécessairement la consultation de plusieurs fournisseurs. Ces différentes exceptions à la procédure d'appel d'offres sont décrites dans le tableau 1.4. L'Algérie ne dispose pas de données sur la fréquence de recours à ces exceptions et le volume qu'elles représentent.

\section{Tableau 1.4. Les exceptions à l'appel d'offres en Algérie}

\begin{tabular}{|c|c|}
\hline \multirow{7}{*}{$\begin{array}{l}\text { Gré à gré simple } \\
\text { (art. } 49 \text { du DMP) }\end{array}$} & Opérateur économique unique \\
\hline & $\begin{array}{l}\text { Urgence impérieuse motivée par un péril menaçant un investissement, un bien du service contractant ou l'ordre } \\
\text { public, ou un danger imminent }\end{array}$ \\
\hline & Approvisionnement urgent destiné à sauvegarder les besoins essentiels de la population \\
\hline & Projet prioritaire et d'importance nationale qui revêt un caractère d'urgence \\
\hline & Promotion de la production et/ou de l'outil national de production \\
\hline & $\begin{array}{l}\text { Lorsqu'un texte législatif ou réglementaire attribue à un établissement public à caractère industriel et } \\
\text { commercial un droit exclusif pour exercer une mission de service public }\end{array}$ \\
\hline & Lorsqu'un appel d'offres est déclaré infructueux pour la deuxième fois \\
\hline \multirow{5}{*}{$\begin{array}{l}\text { Gré à gré après consultation } \\
\text { (art. } 51 \text { du DMP) }\end{array}$} & Lorsqu'un appel d'offres est déclaré infructueux pour la deuxième fois \\
\hline & $\begin{array}{l}\text { Pour les marchés d'études, de fournitures et de services spécifiques dont la nature ne nécessite pas le recours } \\
\text { à un appel d'offres (la spécificité de ces marchés est déterminée par l'objet du marché, le faible degré de } \\
\text { concurrence ou le caractère secret des prestations) }\end{array}$ \\
\hline & Pour les marchés de travaux relevant directement des institutions publiques de souveraineté de l'État \\
\hline & $\begin{array}{l}\text { Pour les marchés déjà attribués faisant l'objet d'une résiliation, et dont la nature ne s'accommode pas avec les } \\
\text { délais d'un nouvel appel d'offres }\end{array}$ \\
\hline & $\begin{array}{l}\text { Pour les opérations réalisées dans le cadre de la stratégie de coopération du gouvernement, ou d'accords } \\
\text { bilatéraux de financement concessionnels, de conversion de dettes en projets de développement ou de dons, } \\
\text { lorsque ces accords de financement le prévoient }\end{array}$ \\
\hline
\end{tabular}

Source : DMP. 


\section{Définir les exceptions à l'appel d'offres avec davantage de précision}

Certaines exceptions à l'appel d'offres figurant aux articles 49 et 51 du DMP ne sont pas définies de façon suffisamment précise, ce qui engendre des risques d'abus. C'est le cas des «projets prioritaires d'importance nationale qui revêtent un caractère d'urgence » et de " la promotion de l'outil national de production », qui donnent lieu à une attribution au gré à gré simple (art. 49 du DMP). Lors des missions exploratoires de l'OCDE, la construction de grands ensembles de logements a été citée comme projet prioritaire d'importance nationale qui revêt un caractère d'urgence. C'est également le cas du gré à gré après consultation (exception à la procédure d'appel d'offres de l'article 51 du DMP) concernant les marchés de travaux relevant directement des institutions publiques de souveraineté de l'État. En effet, la réglementation algérienne ne donne aucune précision quant à la nature des institutions publiques de souveraineté de l'État.

Le cadre réglementaire devrait définir plus précisément, et de façon exhaustive, le périmètre de ces exceptions d'appel d'offres, par exemple par le biais des arrêtés fixant les modalités d'application des articles 49 et 51 du DMP.

\section{Mieux encadrer les conditions de recours aux exceptions à l'appel d'offres}

II est important que le recours aux exceptions à l'appel d'offres soit documenté et dûment justifié par les services contractants. Or, plusieurs des exceptions prévues aux articles 49 et 51 du DMP ne prévoient pas de mécanismes d'encadrement et de contrôles suffisants pour éviter les possibles abus par les services contractants. C'est le cas des exceptions se référant à l'urgence impérieuse ou à un approvisionnement urgent destiné à sauvegarder les besoins essentiels de la population (art. 49 du DMP, voir tableau 1.4), ou encore de l'exception pour des marchés d'études, de fournitures et de services spécifiques dont la nature ne nécessite pas le recours à un appel d'offres (art. 51 du DMP, voir tableau 1.4).

Le ministère des Finances pourrait encadrer les conditions de recours à ces exceptions par arrêté, comme prévu aux articles 49 et 51 du DMP. Le recours à ces exceptions devrait nécessiter la prise d'une décision motivée par le responsable de l'institution publique, dont la copie devrait être obligatoirement transmise à la Cour des comptes et au ministre des Finances (et à l'ARMP, lorsqu'elle sera mise en place). D'autre part, la justification du recours au gré à gré simple devrait être documentée par le service contractant et enregistrée, de façon à ce que l'ARMP ou d'autres corps d'audit et d'inspection puissent exercer un contrôle a posteriori. La justification de l'urgence impérieuse devrait être étrangère aux retards générés par le service contractant lui-même.

La procédure de l'article 23 du DMP est une autre exception au processus d'appel d'offres qui permet de passer des marchés publics portant sur des «produits et services qui, en raison de leur nature, des fluctuations rapides de leur prix et de leur disponibilité, ainsi que des pratiques commerciales qui leur sont applicables, nécessitent une promptitude de décision » (art. 23 du DMP). Aucun arrêté ministériel fixant la liste de ces produits et services n'a été promulgué jusqu'à présent, et l'équipe de l'OCDE ne dispose pas de données quant à une utilisation éventuelle de cette procédure depuis l'entrée en vigueur du DMP en 2015.

En conclusion, l'Algérie devrait considérer l'utilité et la pertinence de la procédure dérogatoire prévue à l'article 23 du DMP. Si cette procédure n'a jamais été utilisée jusqu'à présent, le gouvernement algérien pourrait envisager sa suppression lors de la prochaine réforme du cadre réglementaire des marchés publics. En cas de maintien de cette exception, il conviendrait de s'assurer, d'une part, que le ministère des Finances et l'ARMP soient suffisamment représentés au sein de de la commission interministérielle ad hoc chargée de négocier et de choisir le partenaire cocontractant et, d'autre part, que les mécanismes d'encadrement et de contrôle mentionnés ci-dessus (décision motivée, notification) soient en place. 


\section{Clarifier la portée des exclusions}

Certaines catégories d'achats ou de dépenses sont considérées comme« exclues " du champ d'application du cadre réglementaire des marchés publics (voir encadré 1.2). En effet, la réglementation sur les marchés publics n'est pas appropriée pour régir toutes les catégories d'achat. Ces exclusions concernent généralement des catégories qui impliquent des transactions pour lesquelles le processus de passation de marchés publics « classique » n'est pas adapté. Elles sont régies par leur propre législation ou réglementation, par exemple s'agissant de l'immobilier ou des contrats de travail (OCDE, à paraître $\left.{ }_{[5]}\right)$. Ainsi les pays de l'OCDE ont intégré dans leur cadre réglementaire une liste d'exclusion régissant les marchés publics En Algérie, l'article 7 du DMP fixe la liste des contrats qui ne sont pas soumis au DMP (Encadré 1.8).

\section{Encadré 1.8. Exclusions : contrats publics non soumis au DMP (art. 7)}

- Les contrats passés par les institutions, les administrations publiques et les établissements publics à caractère administratif entre eux.

- Les contrats passés avec les institutions et établissements publics lorsqu'ils exercent une activité qui n'est pas soumise à la concurrence.

- Les contrats de maîtrise d'ouvrage déléguée.

- Les contrats d'acquisition ou de location de terrains ou de biens immobiliers.

- Les contrats passés avec la Banque d'Algérie (banque centrale).

- Les contrats passés en vertu des procédures des organisations et des institutions internationales ou en vertu d'accords internationaux, lorsque cela est requis.

- Les contrats relatifs aux prestations de service de conciliation et d'arbitrage.

- Les contrats passés avec une centrale d'achat soumise aux dispositions du présent titre, agissant pour le compte des services contractants.

Source : DMP.

Certaines exclusions prévues à l'article 7 du DMP pourraient être définies avec plus de précision dans le cadre réglementaire, alors que d'autres pourraient être revues sur la base des bonnes pratiques internationales.

\section{Des contrats passés par les institutions et les administrations publiques, et les établissements publics à caractère administratif entre eux.}

Les contrats passés entre entités publiques font l'objet d'un traitement spécifique dans la réglementation des marchés publics de nombreux pays de l'OCDE. Dans la réglementation européenne, une exclusion de la réglementation des marchés publics est prévue pour les contrats dits "in house », c'est-à-dire passés avec une personne morale sur laquelle le service contractant exerce " un contrôle analogue à celui qu'il exerce sur ses propres services » (Union Européenne, 2014[27]). Au Mexique, les contrats entre organismes publics sont exclus de la sphère d'application de la loi sur les marchés publics, sauf en cas de sous-traitance des prestations par le fournisseur public à une autre partie (OCDE, à paraître $\left.{ }_{[5]}\right)$. Cependant ce type de contrats peut donner lieu à des abus et des fraudes, notamment par le biais de la sous-traitance à des partenaires privés $(\mathrm{OCDE}$, à paraître $[5]$ ). L'exclusion de ces contrats du cadre réglementaire des marchés publics en Algérie devrait donc être plus encadrée. Par exemple, le service contractant devrait être tenu de fournir à l'ARMP une justification détaillée des raisons techniques et financières du choix d'une institution publique en tant que fournisseur, accompagnée d'une étude de marché détaillée. En cas 
de justification insuffisante, l'ARMP devrait pouvoir effectuer des contrôles supplémentaires ou imposer au service contractant l'application des formalités prévues par le DMP.

\section{Des contrats passés avec les institutions et établissements publics lorsqu'ils exercent une activité qui n'est pas soumise à la concurrence}

Cette exclusion est proche de celle évoquée au paragraphe précédent car il s'agit de contrats passés par des institutions ou établissements publics entre eux. Afin d'éviter des abus de cette exclusion, l'Algérie gagnerait à détailler et clarifier les activités non-soumises à la concurrence au sens de l'article 7 du DMP. Cette clarification pourrait faire l'objet d'un arrêté du ministre des Finances, qui reprendrait également les recommandations du paragraphe précédent.

\section{Des contrats passés avec des avocats}

Dans de nombreux pays, les marchés publics de services juridiques font l'objet de procédures concurrentielles, tout en respectant les règles propres à ces professions (OCDE, à paraître $\left[5_{3}\right]$ ). Au Royaume-Uni, par exemple, la centrale d'achat de l'administration centrale (Crown commercial service) a mis en place des accords-cadres multi-attribués pour que les services contractants puissent répondre à leurs besoins en matière de services juridiques tout en garantissant la concurrence (encadré 1.9).

\section{Encadré 1.9. Avantages de l'accord-cadre sur les services juridiques au Royaume-Uni}

Un accord-cadre a été mis en place par le Crown Commercial Service, la centrale d'achat du RoyaumeUni, pour la fourniture de services de conseil juridique pour les entités publiques du gouvernement central. II n'existe pas de valeur limite minimale ou maximale pour l'utilisation de l'accord-cadre ; cependant, les contrats dont la valeur est inférieure à 20000 GBP (livres sterling, environ 23400 EUR) peuvent en être exemptés. Les fournisseurs de l'accord cadre sont divisés en deux catégories selon leur taille et leur degré d'expertise.

L'utilisation de cet accord-cadre a permis aux entités publiques de bénéficier de plusieurs avantages : tarifs compétitifs, facilité d'utilisation et gestion professionnalisée de la relation fournisseur.

Source : Adapté de (Crown Commercial Service, 2017[29]).

Les services juridiques et la représentation juridique sont des secteurs suffisamment concurrentiels pour faire l'objet d'appels d'offres. En effet, l'Algérie compte plus de 30000 avocats, et plusieurs firmes

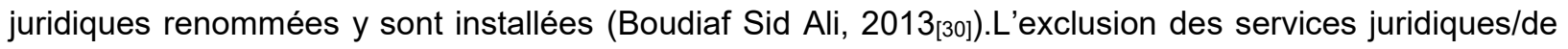
représentation est d'autant moins justifiée que l'article 24 du DMP prévoit la possibilité, pour les services contractants, de recourir à la procédure adaptée s'agissant des prestations juridiques, quels que soient leurs montants (CMS Francis Lefebvre Avocats 2018, 2015[31]).

Afin d'assurer une concurrence adéquate, l'Algérie pourrait donc supprimer l'exclusion des contrats passés avec des avocats et d'autres services juridiques du cadre réglementaire des marchés publics. En effet, les services contractants ont l'option de recourir à la procédure adaptée quel que soit le montant estimé des prestations. Le cas échéant, l'ARMP pourrait mettre en place un contrat-programme pour satisfaire aux besoins des services contractants en matière de prestations juridiques, y compris de représentation et d'assistance par des avocats. 


\subsubsection{Ouvrir les marchés publics à la concurrence internationale, nationale et locale}

L'ouverture des marchés publics à l'ensemble des fournisseurs potentiels est un moyen efficace de stimuler la concurrence. Une concurrence accrue favorise la compétitivité et permet aux pays d'obtenir les meilleurs rapports qualité/prix ainsi qu'une meilleure utilisation des ressources publiques (OMC (Organisation Mondiale du Commerce), 2015[32]). Afin de garantir l'accès et la participation de tous les fournisseurs, y compris étrangers, aux marchés publics, il convient de les traiter de manière équitable, juste et transparente, de veiller à ce que les besoins soient les plus compréhensibles possibles par le tissu économique, et de favoriser l'utilisation de procédures concurrentielles (OCDE, 2015 ${ }_{[2]}$ ).

\section{Évaluer l'accès aux opportunités de marchés publics pour les fournisseurs potentiels étrangers}

La Recommandation du Conseil de l'OCDE sur les marchés publics préconise que les gouvernements doivent traiter les soumissionnaires potentiels de manière équitable, juste et transparente, y compris les fournisseurs potentiels étrangers. Les cadres réglementaires ne doivent donc pas présenter de barrières d'accès aux opportunités de marchés publics pour les fournisseurs potentiels étrangers (OCDE, 2016[3]).

Des mesures pour favoriser « l'achat national » ou d'autres mesures restrictives concernant les marchés publics doivent être utilisées avec précaution. En effet, de telles mesures peuvent :i) exclure les fournisseurs étrangers en réservant les marchés publics aux entreprises domestiques ou en mettant en place une complexité administrative telle qu'elle ne facilite pas l'accès des fournisseurs étrangers aux marchés publics ; ii) s'avérer être, paradoxalement, une cause d'augmentation des coûts ou entraver les activités des entreprises domestiques qui auraient des difficultés pour s'approvisionner sur le marché local et chercheraient des solutions d'approvisionnement à l'étranger; et iii) inciter les autres pays à en faire autant, créant ainsi des barrières à l'export pour les entreprises nationales (OMC (Organisation Mondiale du Commerce), 2011[33]). C'est pourquoi de nombreux pays ont ouvert leurs marchés publics à l'international en signant des accords bilatéraux, régionaux ou encore l'Accord multilatéral sur les marchés publics (AMP) de l'Organisation mondiale du commerce (OMC), dont l'Algérie n'est pas membre (OMC (Organisation Mondiale du Commerce), s.d.[34]). Certains articles du DMP laissent apparaître clairement des barrières aux fournisseurs potentiels étrangers et peuvent avoir un effet négatif sur la perception de la transparence et de l'équité de traitement dans les marchés publics en Algérie (encadré 1.10), et constituer ainsi des barrières à l'accès aux opportunités de marchés publics.

\section{Encadré 1.10. Disposition du DMP visant à favoriser les entreprises de droit algérien}

En Algérie, le DMP contient des articles visant à favoriser les entreprises de droit algérien par rapport aux fournisseurs étrangers, créant des barrières d'accès significatives pour la participation des fournisseurs étrangers aux marchés publics.

L'article 83accorde, dans tous les types d'appel d'offres, une marge de préférence de $25 \%$ au stade de l'évaluation des offres financières aux produits d'origine algérienne ou aux entreprises de droit algérien dont le capital est détenu majoritairement par des nationaux résidents.

L'article 84 mentionne que, dans le cadre des appels d'offres internationaux, les cahiers des charges doivent prévoir que les fournisseurs étrangers s'engagent à investir en partenariat avec un opérateur économique algérien.

Enfin, l'article 85 stipule que si l'outil de production national est en mesure de satisfaire les besoins du service contractant, ce dernier doit lancer un appel à la concurrence national dont seront exclus les fournisseurs étrangers. Cette obligation s'applique à tous les projets dont la liste est déterminée par le 
service contractant ou son ministre de tutelle (dans le cas des établissements publics). En outre, les entreprises étrangères qui soumissionnent seules ont l'obligation de sous-traiter au minimum $30 \%$ du montant du marché à des entreprises algériennes, sauf impossibilité dûment justifiée.

Source : DMP.

L'équipe OCDE a analysé les taux d'appels d'offres internationaux lancés au cours d'une période courte (extraits du Bomop du 14 octobre au 1er décembre 2018) : environ $34 \%$ des appels d'offres étaient de portée internationale, preuve que l'outil de production national ne peut pas répondre au besoin des services contractants pour plus d'un tiers des besoins exprimés sur la période constatée. Cependant, aucune donnée n'est disponible concernant le taux de pénétration des fournisseurs étrangers dans les marchés publics algériens. L'impact sur les fournisseurs étrangers des restrictions à leur égard, imposées dans les articles 83, 84 et 85 mentionnés plus haut, ne peut donc pour le moment être mesuré. L'Algérie devrait mener une évaluation des mesures visant à favoriser les entreprises de droit algérien dans les marchés publics afin d'analyser l'efficacité du système en place.

\section{La création d'EPIC visant à répondre aux besoins publics n'est pas la seule solution pour s'assurer de la bonne exécution de certains marchés publics}

La Recommandation du Conseil de l'OCDE sur les marchés publics encourage les adhérents à engager des dialogues réguliers et transparents avec le tissu économique afin de faire connaître les besoins aux soumissionnaires potentiels, mais également de donner aux pouvoirs adjudicateurs une meilleure connaissance des capacités du marché et ainsi adapter les documents des futurs marchés en conséquence (cahiers des charges, contrats, etc.). Un dialogue régulier entre les parties durant la phase d'exécution d'un contrat permet une identification et une résolution rapides des problèmes (OCDE, 2015[2]).

En Algérie, d'après les missions exploratoires de l'OCDE, afin de pallier les défaillances des fournisseurs existants ou en l'absence de fournisseurs aux capacités adéquates, certaines collectivités territoriales ont créé des établissements publics à caractère industriel et commercial(EPIC). Cette pratique existe, par exemple, dans le domaine de l'entretien des espaces verts ou du nettoyage. La défaillance des fournisseurs existants ou l'absence de fournisseurs aux capacités adéquates peuvent être attribuées à plusieurs facteurs, identifiés dans d'autres chapitres du présent rapport :

1. des analyses de marché insuffisantes (voir chapitre 4),

2. des critères de sélections inadaptés au besoin, un choix trop fréquent de l'offre financière la plus basse, et des cahiers des charges et des contrats non adaptés à la fois aux besoins des pouvoirs adjudicateurs et aux capacités des fournisseurs potentiels (voir chapitre 4),

3. l'absence de fichier national des opérateurs économiques, permettant à l'entité contractante de se renseigner sur les performances des fournisseurs potentiels (voir chapitre 4),

4. des agents publics en charge de la passation des marchés ne disposant pas de capacités suffisantes (chapitre 6) en termes d'aspects "pratiques » de la fonction achat, ce qui ne leur permet pas de construire des marchés répondant pleinement aux besoins de l'entité adjudicatrice, tout en étant équilibrés et adaptés aux capacités du tissu économique.

Le choix de créer un établissement dédié n'est pas à exclure, mais il convient d'en examiner les avantages et les inconvénients. En effet, si les EPIC permettent d'assurer une continuité de service en cas de défaillance du tissu économique, leur mise en place a un coût. Par ailleurs, ces établissements sont soumis au DMP pour leurs propres achats, ce qui impacte leur coût de fonctionnement, financé par les deniers publics, limitant ainsi leur flexibilité. Un autre moyen efficace à disposition des entités adjudicatrices pour 
connaître les capacités du tissu économique est d'entretenir un dialogue régulier et transparent avec les fournisseurs potentiels et les associations professionnelles (voir chapitre 4).

L'Algérie devrait inciter les services contractants à analyser les causes des défaillances du tissu économique afin de limiter un recours systématique à la création d'EPIC comme solution palliative sur le long terme. Cette analyse permettra de rédiger des cahiers des charges et des documents contractuels en adéquation avec les besoins des entités adjudicatrices et les capacités des fournisseurs potentiels.

\section{Intégrer le dialogue compétitif au cadre réglementaire}

Les procédures concurrentielles avec négociation, comme le dialogue compétitif, sont de plus en plus utilisées dans la pratique de certains pays de l'OCDE, notamment dans l'UE (Union Européenne, 2014[27]). Le dialogue compétitif est une procédure concurrentielle ouverte à tout opérateur économique et extrêmement utile si le pouvoir adjudicateur n'est pas en mesure d'évaluer les solutions qu'offre le marché sur les plans technique, financier ou juridique. Le dialogue compétitif permet au pouvoir adjudicateur, après la mise en place d'un programme fonctionnel définissant les objectifs à atteindre et une présélection des opérateurs économiques, de négocier avec chacun d'entre eux sur des points liés au marché. Suite à ces discussions, les candidats remettent une offre formelle finale au pouvoir adjudicateur.

Le dialogue compétitif est notamment utilisé pour certains projets innovants et/ou complexes, tels que la réalisation d'infrastructures de transport intégrées, de grands réseaux informatiques, de travaux qui ne concernent pas des bâtiments standards, etc. II peut également être adapté en cas d'infructuosité de certaines procédures ouvertes ou restreintes, si elles n'ont donné lieu qu'à des offres irrégulières ou inacceptables. Dans ce cas, le dialogue compétitif peut en effet être un instrument pour obtenir des offres régulières et acceptables (OCDE, 2016[35]) (Union Européenne, 2014[27]). L'encadré 1.11 ci-dessous décrit les différentes étapes d'un dialogue compétitif selon la directive sur les marchés publics de l'UE.

\section{Encadré 1.11. Les étapes du dialogue compétitif selon la directive sur les marchés publics de} l'UE.

1. Les pouvoirs adjudicateurs émettent un avis de marché dans lequel figurent les critères de sélection des opérateurs économiques qui participeront au dialogue compétitif. Ils indiquent leurs besoins et leurs exigences dans l'avis de marché et définissent ces besoins et ces exigences dans cet avis et/ou dans un document descriptif. À cette occasion, et dans les mêmes documents, ils indiquent et définissent également les critères d'attribution retenus et fixent un calendrier indicatif.

2. Seuls les opérateurs économiques ayant reçu une invitation du pouvoir adjudicateur à la suite de l'évaluation des informations fournies peuvent participer au dialogue. Les pouvoirs adjudicateurs peuvent limiter le nombre de candidats admis à présenter une offre qui seront invités à participer à la procédure.

3. Les pouvoirs adjudicateurs ouvrent un dialogue dont l'objet est l'identification et la définition des moyens propres à satisfaire au mieux leurs besoins. Les dialogues compétitifs peuvent se dérouler en phases successives de manière à réduire le nombre de solutions à discuter pendant la phase du dialogue en appliquant les critères d'attribution énoncés dans l'avis de marché ou dans le document descriptif. Aucune information sur les solutions des candidats n'est révélée durant les discussions.

4. Le pouvoir adjudicateur poursuit le dialogue jusqu'à ce qu'il soit en mesure d'identifier la ou les solutions qui sont susceptibles de répondre à ses besoins. 
5. Après avoir prononcé la clôture du dialogue et en avoir informé les participants restant en lice, les pouvoirs adjudicateurs invitent chacun d'eux à soumettre leur offre finale sur la base de la ou des solutions présentées et spécifiées au cours du dialogue. Ces offres comprennent tous les éléments requis et nécessaires pour la réalisation du projet.

6. Les pouvoirs adjudicateurs évaluent les offres en fonction des critères d'attribution fixés dans l'avis de marché ou dans le document descriptif. Des négociations peuvent être menées avec le soumissionnaire reconnu comme ayant remis l'offre présentant le meilleur rapport qualité/prix.

7. À noter que les pouvoirs adjudicateurs peuvent prévoir des primes ou des paiements au profit des participants au dialogue.

Source : adapté de (Union Européenne, 2014[27]).

Le DMP algérien ne prévoit pas le dialogue compétitif, mais il prévoit une procédure d'appel d'offres restreint (voir section 1.2.2 ci-dessus) à deux étapes, qui peut être utilisée lorsque le service contractant n'est pas en mesure de définir les moyens techniques pour répondre à ses besoins (art. 46 du DMP). Cependant, cette procédure ne permet pas de réelle négociation avec les opérateurs économiques. En effet, la négociation est prohibée dans la procédure d'appel d'offres (art. 80 du DMP). Dès lors, l'Algérie gagnerait à prévoir dans le DMP une procédure ouverte à l'ensemble des opérateurs économiques et permettant une réelle négociation, inspirée du dialogue compétitif.

\subsection{Des processus et une documentation favorisant la concurrence}

Afin de faciliter l'accès pour les concurrents potentiels aux opportunités des marchés publics, il convient de fournir, dans le cadre des appels d'offres, une documentation claire, synthétique, aussi normalisée que possible et adaptée aux besoins. II s'agit de s'assurer que : i) chaque appel d'offres soit conçu de façon à encourager un large éventail de concurrents potentiels à y répondre ce qui implique de fournir des orientations claires sur les attentes des acheteurs ( $y$ compris en termes de cahier des charges, de contrat et de modalités de paiement); ii) l'ampleur et la complexité des renseignements exigés dans l'appel d'offres, et le temps imparti aux fournisseurs pour répondre ne soient pas disproportionnés par rapport à la taille et à la complexité du marché public (OCDE, 2015[2]).

À cette fin, l'OCDE préconise la mise en place de modèles que les agents en charge de la passation des marchés publics pourront utiliser dans le cycle de passation d'un marché public. Ces modèles incluent les modèles d'avis d'appel d'offres, de documents d'appel d'offres, ou encore de contrats. Ces documents devront contenir les clauses standards et obligatoires ou être des modèles représentatifs du cadre légal et des conditions contractuelles standards pour les types de contrats les plus courants (OCDE, 2016[3]). Leur mise en place simplifie la compréhension de l'ensemble des pièces du cycle de passation des marchés publics, encourageant ainsi les concurrents de toute taille à y participer, y compris les nouveaux entrants et les PME. Cela permet également de limiter les erreurs qui pourraient être commises par les soumissionnaires potentiels comme par les agents en charge de la passation des marchés publics, permettant ainsi à l'ensemble du système d'être clair, transparent, efficace et équitable. (OCDE, 2016[3]) 


\subsubsection{Encourager la concurrence grâce à une documentation normalisée, clarifiée et simplifiée}

\section{Standardiser les documents d'appel d'offres pour favoriser et uniformiser l'accès aux marchés publics}

Afin d'encourager la participation d'un maximum de soumissionnaires, les attentes des entités adjudicatrices (notamment les spécifications techniques) et les modalités de soumission (comprenant les critères d'attribution et leur pondération) doivent être clairement rédigés. Pour cela, les agents en charge de la passation des marchés publics doivent disposer d'outils pour les guider dans l'élaboration de la documentation des appels d'offres. Ces outils peuvent être notamment des modèles de documents (par exemple des modèles de documentation d'appel d'offres comprenant les exigences minimales attendues, conformes aux exigences du cadre réglementaire des marchés publics) (OCDE, 2016[3]).

L'utilisation de modèles de documents standardisés permet également de sécuriser, clarifier et uniformiser les procédures, et ce, pour les entités adjudicatrices comme pour les soumissionnaires (OCDE, 2013 [36]). De nombreux pays ont pris conscience de l'importance de la standardisation de documents relatifs aux marchés publics, comme par exemple l'Irlande (voir encadré 1.12).

\section{Encadré 1.12. Le développement d'une documentation standardisée d'appel d'offres en Irlande}

Le Bureau des achats du gouvernement d'Irlande (OGP, son acronyme en anglais) est responsable de la rédaction des politiques des achats publics et réglemente et coordonne le système national d'achats publics. De plus, l'OGP est chargé de la préparation de documents standardisés d'appel d'offres et de guides.

L'OGP a élaboré un ensemble de modèles de documents d'appel d'offres afin de dicter les bonnes pratiques à mettre en œuvre dans le respect des directives européennes de 2014 en matière d'achat public. Ces modèles sont disponibles sur le site internet de l'OGP. II existe actuellement six modèles de documents et davantage sont prévus. Ils couvrent les phases de procédures d'appel d'offres et d'exécution des contrats.

À ce jour, ces documents ont été utilisés par les services publics dans le cadre d'achats de biens et de services comportant des risques faibles à intermédiaires. Ils permettent également de professionnaliser, moderniser et standardiser la fonction achat au sein de l'ensemble des entités adjudicatrice en Irlande. Leur popularité réside principalement dans leur clarté et leur facilité d'utilisation.

Source : (OCDE, 2018[37]).

En Algérie, il n'existe pas de documents d'appels d'offres standards, chaque entité adjudicatrice développe ses propres documents. L'article 213 du DMP prévoit que l'ARMP soit chargée d'informer, diffuser et vulgariser tous documents ou informations relatifs aux marchés publics et délégations de service public ; il lui revient donc de standardiser tous documents relatifs à la passation de marché public. L'Algérie verrait son système de passation des marchés publics renforcé et l'accès aux opportunités de marchés publics accru si des modèles de documents standards d'appels d'offres, y compris les contrats, à disposition des entités adjudicatrices du pays étaient élaborés. L'ARMP doit donc être mise en place dans les meilleurs délais. 


\section{Développer des conditions contractuelles standards pour certains types d'achats sous le pilotage de l'ARMP}

En plus de modèles standards de documents d'appels d'offres, l'accès aux opportunités de marchés publics peut être élargi et renforcé en développant des conditions contractuelles standards pour les contrats les plus courants (OCDE, 2016 $\left.6_{[3]}\right)$. L'utilisation systématique et généralisée de modèles de contrats efficaces permet de renforcer la clarté, les capacités, la sécurisation et l'harmonisation des pratiques, aussi bien pour l'entité adjudicatrice que pour le secteur privé, et cela réduit également les risques durant la phase contractuelle (OECD, 2009[38]). Conscients de ces bénéfices sur leur système de passation des marchés publics, de nombreux pays ont développé un ensemble préétabli de clauses standards, comme par exemple la Nouvelle-Zélande, qui a développé ce type de modèles pour les contrats liés à des achats réguliers du gouvernement (encadré 1.13).

\section{Encadré 1.13. Modèles de contrats du gouvernement de Nouvelle-Zélande}

Le ministère du Commerce, de l'Innovation et du Travail (MBIE, son acronyme en anglais) a développé un ensemble préétabli de clauses standards pour les contrats liés à des achats réguliers du gouvernement, appelées "modèles de contrat du gouvernement » (GMC en anglais). Le développement et la mise en œuvre des GMC fait partie du programme de réforme des achats du gouvernement et a été commandité par une directive demandant au MBIE de « créer un ensemble clair, dans un langage simple, de clauses standards pour les contrats courants de biens et services, qui seront utilisés par tous les services publics et les services de l'État ».

Ces standards concernent les biens et services de faible valeur et de faible risque. Chaque entité utilisant ces GMC a la responsabilité de définir ce qu'elle entend comme étant des biens et services de faible valeur et faible risque. Grâce à l'utilisation de ces GMC dans l'ensemble des services publics et des services de l'État, le gouvernement a pour objectif de :

- Fournir des contrats simples et clairs, faciles d'utilisation pour les agences comme pour les fournisseurs.

- Équilibrer les risques entre l'acheteur public et le fournisseur.

- Standardiser le traitement légal du risque pour les contrats de faible valeur et de faible risque.

- Réduire les négociations et les conseils juridiques pour les achats courants.

- Promouvoir des pratiques homogènes.

- Promouvoir des efficiences dans le processus contractuel de grand volume à faible coût transactionnel.

- Simplifier les opportunités d'affaires avec le gouvernement.

- Renforcer les bonnes pratiques en matière d'achat, conformément aux meilleures pratiques internationales.

Source : (OCDE, 2016[39]).

L'Algérie dispose d'un modèle de clauses standards pour un type de contrat courant, le cahier des clauses administratives générales (CCAG) pour les marchés de travaux publics, approuvé par l'arrêté du 24 novembre 1964. D'après les missions exploratoires, ce CCAG n'a jamais été mis à jour, de plus il n'existe pas d'autre modèle de contrat standard, notamment pour les marchés publics de fournitures, de services et d'études. 
Par conséquent, l'Algérie devrait envisager la mise à jour de son CCAG concernant les travaux publics mais également le développement d'autres CCAG pour les achats courants de fournitures et de services. La création de ces contrats standards devra être pilotée par l'ARMP dont la mise en place doit être effective dans les meilleurs délais.

\subsubsection{Faciliter l'accès aux marchés publics en augmentant la durée de préparation des offres}

L'un des éléments essentiels à considérer pour la mise en application des meilleures pratiques internationales est de s'assurer que les délais octroyés aux soumissionnaires pour préparer leurs offres sont correctement dimensionnés. Pour cela, il est nécessaire de prendre en compte la nature et la complexité de la procédure d'achat afin d'adapter la période durant laquelle les soumissionnaires pourront retirer les documents de consultation et soumettre une offre. Il est tout aussi important de définir des délais minimums pour la soumission des offres ou propositions pour chaque mode de passation des marchés publics, et de prévoir des extensions de ces délais lors de mises en concurrence internationales (OCDE, $\left.2016_{[3]}\right)$. Cela permet non seulement d'accroître le nombre de soumissionnaires, mais aussi de favoriser la concurrence (OCDE, 2018[1]).

Le DMP n'impose pas de délai minimum aux soumissionnaires pour la préparation des offres, et ce, quel que soit le mode de passation. En effet, seul l'article 66 du DMP mentionne le délai de préparation des offres, qui doit être fixé « en fonction d'éléments tels que la complexité de l'objet du marché projeté et le temps normalement nécessaire pour la préparation des offres et leur acheminement », et qui doit " permettre à la concurrence, la plus large possible, de jouer pleinement ». Le service contractant est donc libre de choisir le délai qu'il laissera aux soumissionnaires pour préparer leurs offres. De plus, en analysant des avis d'appel d'offres publiés dans le Bomop pour la période du 18 au 24 novembre 2018, il a été constaté que les délais étaient disparates (voir tableau 1.5), et inférieurs à ceux préconisés par les bonnes pratiques internationales, par exemple celles de l'UE (encadré 1.14). Par ailleurs, les délais minimums constatés, notamment pour les appels d'offres nationaux et internationaux ouverts ( 15 jours) et pour les appels d'offres nationaux ouverts et nationaux ouverts avec exigences de capacités minimales (10 jours), ne sont pas suffisants pour permettre une participation et un accès transparents et efficients aux marchés publics et favoriser la concurrence.

Tableau 1.5. Délais de soumission constatés pour les appels d'offres en Algérie

\begin{tabular}{|c|c|c|c|}
\hline Type d'appel d'offres & Délai moyen constaté (en jours) & $\begin{array}{c}\text { Délai minimum constaté } \\
\text { (en jours) }\end{array}$ & $\begin{array}{c}\text { Délai maximum constaté } \\
\text { (en jours) }\end{array}$ \\
\hline National et international ouvert & 28 & $\begin{array}{c}15 \\
\text { ANEP } n^{\circ} 220931\end{array}$ & $\begin{array}{c}35 \\
\text { ANEP } n^{\circ} 220941\end{array}$ \\
\hline $\begin{array}{l}\text { National et international ouvert } \\
\text { avec exigences de capacités } \\
\text { minimales }\end{array}$ & 45 & $\begin{array}{c}30 \\
\text { ANEP } n^{\circ} 220899\end{array}$ & $\begin{array}{c}60 \\
\text { ANEP } n^{\circ} 833825\end{array}$ \\
\hline National et international restreint & 60 & 60 & 60 \\
\hline National ouvert & 29 & $\begin{array}{c}10 \\
\text { ANEP } n^{\circ} 833754\end{array}$ & $\begin{array}{c}60 \\
\text { ANEP } n^{\circ} 833787\end{array}$ \\
\hline $\begin{array}{l}\text { National ouvert avec exigences de } \\
\text { capacités minimales }\end{array}$ & 18 & $\begin{array}{c}10 \\
\text { ANEP } n^{\circ} 833782\end{array}$ & $\begin{array}{c}30 \\
\text { ANEP } n^{\circ} 25015088\end{array}$ \\
\hline
\end{tabular}

Source : Bomop, semaine du 18 au 24 novembre 2018. 


\section{Encadré 1.14. Règles de l'UE concernant les délais de soumission pour les appels d'offres}

\section{Procédure ouverte:}

Le délai minimum de soumission des offres est de $\mathbf{3 5}$ jours à compter de la date de publication de l'avis de marché. Si un avis de pré-information a été publié, ce délai peut être ramené à 15 jours.

\section{Procédure restreinte :}

Toute entreprise peut demander à participer à une procédure restreinte, mais seules celles qui sont présélectionnées seront invitées à soumettre une offre. Le délai pour demander à participer est de 37 jours à compter de la date de publication de l'avis de marché. L'autorité publique sélectionne ensuite au moins 5 candidats possédant les capacités requises. Ceux-ci peuvent alors soumettre une offre dans les $\mathbf{4 0}$ jours suivant la date d'envoi de l'invitation à soumissionner. Ce délai peut être ramené à 36 jours si un avis de pré-information a été publié. En cas d'urgence, une autorité publique peut fixer un délai de 15 jours pour recevoir les demandes de participation (10 jours si l'avis est envoyé par voie électronique) et un délai de 10 jours pour la soumission des offres.

Source : (Union Européenne, s.d.[40]).

Dans la pratique, les soumissionnaires potentiels peuvent demander une prolongation du délai de soumission auprès du service contractant, ce qui est une pratique courante d'après les échanges avec les autorités algériennes. Cependant, les échanges durant les missions exploratoires montrent que de telles prolongations ne sont pas octroyées fréquemment. Afin de favoriser l'accès aux opportunités des marchés publics et pour encourager la concurrence, l'Algérie devrait envisager l'introduction dans son cadre réglementaire de délais minimums de préparation des offres pour les appels d'offres ouverts et restreints, nationaux ou internationaux.

\subsubsection{Assurer aux fournisseurs des délais de paiement encadrés par la réglementation}

La mise en place d'un cadre réglementaire imposant aux pouvoirs adjudicateurs des délais de paiement des fournisseurs constitue une étape essentielle dans la modernisation des relations entre les pouvoirs adjudicateurs et leurs fournisseurs En effet, des délais de paiements trop longs ou non explicitement encadrés peuvent avoir des conséquences quant à leur participation aux marchés publics : fournir des indications claires concernant les délais et conditions de paiement permet de favoriser une participation étendue des fournisseurs potentiels, y compris des nouveaux entrants et des PME. (OCDE, 2015[2]).

En Algérie, le DMP n'encadre pas les délais de paiement. Seuls les délais de mandatement sont encadrés par l'article 122 qui fixe les délais de mandatement entre 30 jours et 2 mois, et les éléments collectés durant les missions exploratoires de l'OCDE ont révélé que le délai de paiement moyen observé était de 90 jours, au-delà des bonnes pratiques observées dans d'autres systèmes de passation des marchés publics. Ainsi, l'UE fixe les délais de paiement à un maximum de 30 jours pour le cas général, et jusqu'à 60 jours pour les EPIC ou les établissements de santé (Union Européenne, 2014[27]).

Par ailleurs, l'article 143 du DMP autorise le paiement direct des sous-traitants, ce qui est conforme aux bonnes pratiques internationales, et notamment celle de l'UE. Le paiement direct des sous-traitants est un élément primordial pour permettre l'accès aux PME aux opportunités de marchés publics (voir chapitre 5).

L'accès des fournisseurs potentiels aux marchés publics serait encouragé si des délais de paiements maximum étaient imposés aux pouvoirs adjudicateurs algériens et inscrits dans le DMP. En réglementant les délais, le système de passation des marchés publics dans son ensemble se verrait renforcé grâce à 
une confiance accrue des fournisseurs potentiels envers les pouvoirs adjudicateurs. De plus, l'Algérie verrait sa bonne pratique de paiement direct des sous-traitants renforcée si les délais maximums s'appliquaient également dans ce cas. Par ailleurs, la mise en place d'outils de passation électronique des marchés publics (voir chapitre 3 ) permettrait de diminuer les délais de paiement, et l'encadrement des délais de paiement favoriserait l'accomplissement des politiques publiques évoquées dans le chapitre 5 .

De plus, les missions exploratoires de l'OCDE ont mis en lumière des délais de paiement importants une fois l'ordre de mandatement reçu, à cause d'un manque de trésorerie ou pour des raisons administratives. En Algérie, le défaut de mandatement dans les délais prévus par le DMP donne droit au versement d'intérêts moratoires aux fournisseurs lésés. Si ces dispositions sont bien prévues par la réglementation (art. 122 du DMP), il semble cependant que peu de fournisseurs fassent valoir leur droit, par craintes de représailles de la part des entités adjudicatrices. Des délais de paiement trop longs et des intérêts moratoires non versés peuvent avoir des effets néfastes sur les fournisseurs, qui pourraient être financièrement fragilisés ou décider de ne plus répondre aux opportunités des marchés publics (OCDE, $\left.2013_{[36]}\right)$. 


\begin{tabular}{|c|c|c|c|}
\hline Résumé des Recommandations & $\begin{array}{l}\text { Court } \\
\text { terme }\end{array}$ & $\begin{array}{c}\text { Moyen } \\
\text { terme }\end{array}$ & $\begin{array}{l}\text { Long } \\
\text { terme }\end{array}$ \\
\hline $\begin{array}{l}\text { Mettre en application l'ensemble des dispositions prévues par le DMP (y compris les articles 88, 89, 203, } \\
204,205,206,212 \text { et } 214 \text { ) }\end{array}$ & $\mathrm{X}$ & & \\
\hline Actualiser les arrêtés de l'ancien cadre réglementaire & $\mathrm{X}$ & & \\
\hline Publier les arrêtés complémentaires & $\mathrm{X}$ & & \\
\hline $\begin{array}{l}\text { Mettre en ligne une présentation hiérarchisée et exhaustive du cadre règlementaire des marchés publics } \\
\text { (DMP et arrêtés d'application) }\end{array}$ & $\mathrm{X}$ & & \\
\hline $\begin{array}{l}\text { Lister les EPE, s'assurer de la nécessité de développer leurs propres cadres réglementaires pour toutes } \\
\text { les catégories d'achat et les encourager à consulter les autorités en charge des marchés publics afin de } \\
\text { s'assurer que leur cadre réglementaire respecte les principes des marchés publics }\end{array}$ & & $\mathrm{X}$ & \\
\hline $\begin{array}{l}\text { Créer une rubrique sur le site web de la Division des marchés publics synthétisant ses avis juridiques } \\
\text { sous forme de « question - réponse » }\end{array}$ & $\mathrm{X}$ & & \\
\hline Clarifier le rôle institutionnel de l'ARMP en matière de délégation de service public & $X$ & & \\
\hline $\begin{array}{l}\text { Mettre en place l'ARMP ou toute autre entité en charge des fonctions de bases centrales et } \\
\text { complémentaires }\end{array}$ & $\mathrm{X}$ & & \\
\hline $\begin{array}{l}\text { Confier de façon provisoire certaines fonctions de l'ARMP à la Division des marchés publics en attendant } \\
\text { sa mise en place }\end{array}$ & $\mathrm{X}$ & & \\
\hline Consultation des parties prenantes avant toute modification du cadre réglementaire des marchés publics & & & $\mathrm{X}$ \\
\hline $\begin{array}{l}\text { Publier des comptes rendus des échanges (ou un résumé des retours) sur les projets de modifications du } \\
\text { cadre réglementaire }\end{array}$ & & & $\mathrm{X}$ \\
\hline $\begin{array}{l}\text { Utiliser la méthode } \mathrm{RACl} \text { pour assigner clairement les responsabilités des différents acteurs institutionnels } \\
\text { et piloter les réformes du système de passation des marchés publics }\end{array}$ & & & $\mathrm{X}$ \\
\hline $\begin{array}{l}\text { Mener des analyses pour s'assurer que les seuils de passation permettent d'atteindre un niveau de } \\
\text { concurrence adéquat tout en prenant en compte les coûts associés à ce formalisme. }\end{array}$ & & $\mathrm{X}$ & \\
\hline Publier un arrêté ou une circulaire apportant plus de précisions relatives aux procédures adaptées & $\mathrm{X}$ & & \\
\hline Publier (ARMP ou ministère des Finances) des « procédures internes types » & $\mathrm{X}$ & & \\
\hline $\begin{array}{l}\text { Rendre obligatoire la publication (Bomop ou plateforme électronique) les avis de consultation pour les } \\
\text { procédures adaptées (article } 14 \text { du DMP) }\end{array}$ & $\mathrm{X}$ & & \\
\hline $\begin{array}{l}\text { Rendre obligatoire la publication d'un avis d'appel à la concurrence dans le cadre de tous les appels } \\
\text { d'offres restreints }\end{array}$ & & & $\mathrm{X}$ \\
\hline Prévoir un nombre minimal de candidats invités à soumissionner aux appels d'offres restreints & & & $X$ \\
\hline $\begin{array}{l}\text { Définir plus précisément, et de façon exhaustive, le périmètre des exceptions au recours à l'appel d'offres } \\
\text { figurant aux articles } 49 \text { et } 51 \text { du DMP }\end{array}$ & $\mathrm{X}$ & & \\
\hline Encadrer les conditions de recours aux exceptions à l'appel d'offres figurant aux articles 49 et 51 du DMP & $\mathrm{X}$ & & \\
\hline $\begin{array}{l}\text { Évaluer la pertinence de la procédure dérogatoire prévue à l'article } 23 \text { et la supprimer le cas échéant, ou } \\
\text { introduire des mécanismes d'encadrement }\end{array}$ & & $\mathrm{X}$ & \\
\hline Encadrer davantage les contrats passés entre entités publiques & $\mathrm{X}$ & & \\
\hline Détailler et clarifier les activités non soumises à la concurrence au sens de l'article 7 du DMP & $\mathrm{X}$ & & \\
\hline $\begin{array}{l}\text { Supprimer l'exclusion des contrats passés avec des avocats et des autres prestations juridiques du cadre } \\
\text { réglementaire des marchés publics. }\end{array}$ & & & $X$ \\
\hline $\begin{array}{l}\text { Mener une évaluation des mesures visant à favoriser les entreprises de droit algérien dans les marchés } \\
\text { publics }\end{array}$ & & $\mathrm{X}$ & \\
\hline $\begin{array}{l}\text { Analyser les causes des défaillances du tissu économique afin de limiter un recours systématique à la } \\
\text { création d'EPIC comme solution palliative }\end{array}$ & & $\mathrm{X}$ & \\
\hline Prévoir dans le DMP une procédure inspirée du dialogue compétitif & & & $\mathrm{X}$ \\
\hline $\begin{array}{l}\text { Élaborer et mettre à disposition des entités des modèles de documents standards d'appels d'offres, y } \\
\text { compris les contrats }\end{array}$ & & $\mathrm{X}$ & \\
\hline Mettre à jour du CCAG concernant les travaux publics & & $\mathrm{X}$ & \\
\hline Développer des CCAG pour les fournitures courantes et les services & & $\mathrm{X}$ & \\
\hline $\begin{array}{l}\text { Introduire des délais de préparation des offres minimums pour les appels d'offres ouverts et retreints, } \\
\text { nationaux et internationaux }\end{array}$ & & & $\mathrm{X}$ \\
\hline $\begin{array}{l}\text { Prévoir dans le DMP des délais de paiement maximum aux pouvoirs adjudicateurs, pour les fournisseurs } \\
\text { comme pour les sous-traitants }\end{array}$ & & & $\mathrm{X}$ \\
\hline
\end{tabular}

Note : Court terme : recommandation à mettre en application dans les 12 mois suivants ; Moyen terme : recommandation à mettre en application dans les 1-2 ans ; Long terme : recommandation à mettre en application dans plus de 2 ans. 


\section{Références}

Boudiaf Sid Ali (2013), Rapport sur la situation de la profession d'avocat en Algérie, http://forumdesdemocrates.over-blog.com/article-rapport-sur-la-situation-de-la-profession-davocat-en-algerie-119781829.html (consulté le 5 juillet 2019).

CMS Francis Lefebvre Avocats 2018 (2015), Algérie : Nouvelle réglementation des marchés publics, http://www.lexplicite.fr/algerie-nouvelle-reglementation-marches-publics/ (consulté le 5 juillet 2019).

Crown Commercial Service (2017), General Legal Advice Services, https://ccsagreements.cabinetoffice.gov.uk/contracts/rm3786 (consulté le 18 juillet 2018).

Direction des affaires juridiques, France (2016), Marchés à procédure adaptée, https://www.economie.gouv.fr/files/files/directions services/daj/marches publics/conseil ach eteurs/fiches-techniques/mise-en-oeuvre-procedure/marches-procedure-adaptee-2016.pdf (consulté le 18 février 2019).

Direction des affaires juridiques, ministère des Finances, France (2018), Rapport annuel 2017, https://www.economie.gouv.fr/files/files/directions services/daj/publications/rapports-activitedaj/rapport-activite-2017.pdf (consulté le 26 février 2019).

EBRD (2009), EBRD Core Principles on an Efficient Public Procurement Framework, https://webcache.googleusercontent.com/search?q=cache:jcgEpf5 OCkJ:https://www.ebrd.c om/documents/legal-reform/core-principles-for-publicprocurement.pdf+\&cd=3\&hl=fr\&ct=clnk\&gl=fr (consulté le 25 février 2019).

Estache, A. et limi A. (2008), Procurement efficiency for infrastructure development and financial needs reassessed., http://dx.doi.org/10.1596/1813-9450-4662.

JORT (2014), Réglementation des Marchés Publics, https://www.droitafrique.com/uploads/Tunisie-Code-marches-publics-2014.pdf (consulté le 12 mars 2019).

Knack, S., N. Biletska et K. Kacker (2017), « Deterring kickbacks and encouraging entry in public procurement markets : evidence from firm surveys in 88 developing countries ", http://documents.worldbank.org/curated/en/817871496169519447/Deterring-kickbacks-andencouraging-entry-in-public-procurement-markets-evidence-from-firm-surveys-in-88developing-countries (consulté le 13 mars 2019).

OCDE (2019), Public Procurement in Malta: Re-engineering the Department of Contracts, OCDE, Paris, https://www.oecd.org/governance/public-procurement/publications/publicprocurement-in-malta.pdf (consulté le 15 mars 2019).

OCDE (2018), Data from the 2018 OECD Survey on the Implementation of the 2015 OECD Recommendations on Public Procurement (Unpublished survey).

OCDE (2018), Politique de la réglementation: Perspectives de l'OCDE 2018, Éditions OCDE, Paris, https://dx.doi.org/10.1787/9789264305458-fr. 
OCDE (2018), Procurement Review of the Chamber of Commerce of Bogotá, Colombia: Aligning Practices with the OECD Public Procurement Recommendation, OCDE, Paris, https://www.oecd.org/gov/public-procurement/publications/procurement-review-chambercommerce-bogota.pdf.

OCDE (2018), Public Procurement in Nuevo León, Mexico : Promoting Efficiency through Centralisation and Professionalisation, Examens de l'OCDE sur la gouvernance publique, Éditions OCDE, Paris, https://dx.doi.org/10.1787/9789264288225-en.

OCDE (2018), Second Public Procurement Review of the Mexican Institute of Social Security (IMSS) : Reshaping Strategies for Better Healthcare, Examens de l'OCDE sur la gouvernance publique, Éditions OCDE, Paris, https://dx.doi.org/10.1787/9789264190191-en.

OCDE (2017), Enhancing the Contributions of SMEs in a Global and Digitalised Economy, OCDE, Paris, https://www.oecd.org/mcm/documents/C-MIN-2017-8-EN.pdf.

OCDE (2016), Checklist for Supporting the Implementation of OECD Recommendation of the Council on Public Procurement: Access, OCDE, Paris, http://www.oecd.org/governance/procurement/toolbox/search/Checklist\%2003\%20Access.pdf (consulté le 28 novembre 2018).

OCDE (2016), Checklist for Supporting the Implementation of the OECD Recommendation of the Council on Public Procurement, OCDE, Paris, http://www.oecd.org/governance/procurement/toolbox/search/checklist-implementation-oecdrecommendation.pdf (consulté le 31 janvier 2019).

OCDE (2016), Data from the 2016 OECD Survey on public procurement (Unpublished survey), Éditions OCDE, Paris.

OCDE (2016), Government Model Contracts in New Zealand, http://www.oecd.org/governance/procurement/toolbox/search/government-model-contractsnew-zealand.pdf (consulté le 5 février 2019).

OCDE (2016), Prévention de la corruption dans les marchés publics, https://www.oecd.org/gov/public-procurement/publications/prevention-corruption-marchespublics.pdf (consulté le 28 décembre 2018).

OCDE (2015), Recommandation du Conseil de l'OCDE sur les marchés publics, OCDE, Paris, http://www.oecd.org/fr/gov/ethique/Recommandation-OCDE-sur-les-marches-publics.pdf (consulté le 16 août 2018).

OCDE (2013), « Organising Central Public Procurement Functions », SIGMA Public Procurement Briefs, $\mathrm{n}^{\circ} 26$, Éditions OCDE, Paris, https://dx.doi.org/10.1787/5js4vmn5s5kden.

OCDE (2013), Public Procurement Review of the State's Employees' Social Security and Social Services Institute in Mexico, Examens de l'OCDE sur la gouvernance publique, Éditions OCDE, Paris, https://dx.doi.org/10.1787/9789264197305-en.

OCDE (2011), « Below Threshold Contracts », SIGMA Public Procurement Briefs, n 15, Éditions OCDE, Paris, https://dx.doi.org/10.1787/5js4vmp1xxd3-en. 
OCDE (2011), From Lessons to Principles for the Use of Public-Private Partnerships (32ndmeeting of the Network of Senior Budget Officials Luxembourg, 6-7 June), http://www.oecd.org/gov/budgeting/48144872.pdf.

OCDE (2006), MAPS, http://www.oecd.org/gov/ethics/37390076.pdf (consulté le 31 janvier 2019).

OCDE (à paraître), Enhancing the Use of Competitive Tendering in Costa Rica's Public Procurement System, OCDE, Paris.

OECD (2009), OECD Principles for Integrity in Public Procurement, OECD Publishing, Paris, https://dx.doi.org/10.1787/9789264056527-en.

OMC (Organisation Mondiale du Commerce) (2015), Ouvrir les marchés et promouvoir la bonne gouvernance, https://www.wto.org/french/thewto f/20y f/gpa brochure2015 f.pdf (consulté le 13 mars 2019).

OMC (Organisation Mondiale du Commerce) (2011), Avant propos : Le régime des marchés publics de l'OMC : Problèmes et réforme, http://docsonline.wto.org/ (consulté le 13 mars 2019).

OMC (Organisation Mondiale du Commerce) (s.d.), L'Accord plurilatéral sur les marchés publics (AMP), https://www.wto.org/french/tratop f/gproc f/gp gpa f.htm (consulté le 13 mars 2019).

Sakakibara, M. et M. Porter (2001), « Competing at Home to Win Abroad: Evidence from Japanese Industry », The Review of Economics and Statistic, vol. Vol. 83, pp. pp. 310-322,, https://www.mitpressjournals.org/doi/pdf/10.1162/00346530151143842.

Santos, J. (2018), Understanding Responsibility Assignment Matrix (RACI Matrix), Projectmanagement.com, https://project-management.com/understanding-responsibility-assignmentmatrix-raci-matrix/ (consulté le 9 janvier 2019).

U.S. Department of State (2018), Investment Climate Statements for 2018- Algeria, https://www.state.gov/e/eb/rls/othr/ics/investmentclimatestatements/index.htm?year=2018\&dli $\mathrm{d}=281653$ \#wrapper (consulté le 25 février 2019).

Union Européenne (2017), Règles et procédures en matière de marchés publics en Europe, https://europa.eu/youreurope/business/selling-in-eu/public-contracts/rulesprocedures/index fr.htm (consulté le 19 mars 2019).

Union Européenne (2014), Directive 2014/24/UE sur la passation des marchés publics, https:/leur-lex.europa.eu/legal-content/FR/TXT/PDF/?uri=CELEX:32014L0024\&from=SL (consulté le 8 mars 2019).

Union Européenne (2014), Directive 2014/25/UE relative à la passation de marchés par des entités opérant dans les secteurs de l'eau, de l'énergie, des transports et des services postaux, https://eur-lex.europa.eu/legalcontent/FR/TXT/PDF/?uri=CELEX:32014L0025\&from=GA (consulté le 25 février 2019).

Union Européenne (s.d.), Règles et procédures en matière de marchés publics en Europe L'Europe est à vous, https://europa.eu/youreurope/business/selling-in-eu/publiccontracts/rules-procedures/index fr.htm (consulté le 30 janvier 2019). 
Ware G. et al. (2007), « Corruption in public procurement: A perennial challenge », dans The Many [28] faces of corruption : tracking vulnerabilities at sector level, The World Bank, Washington DC, http://documents.worldbank.org/curated/en/571831468315566390/pdf/399850REPLACEM10 1OFFICIALOUSE0ONLY1.pdf (consulté le 13 mars 2019). 

2

\section{Préserver l'intégrité du système de} passation des marchés publics en

\section{Algérie}

Le renforcement de l'intégrité dans les marchés publics contribue à une gestion efficace et efficiente des ressources publiques et, par là même, de l'argent des contribuables. Ce chapitre analyse les forces et les opportunités d'amélioration du système d'intégrité des marchés publics en Algérie. II traite d'abord de l'élaboration d'une approche stratégique du système d'intégrité, puis du développement d'une culture d'intégrité chez les parties prenantes, et enfin des dispositifs de surveillance et de contrôle des marchés publics intégrant des stratégies de management des risques. II fournit des recommandations au pays visant à améliorer le système en place.

Les données statistiques concernant Israël sont fournies par et sous la responsabilité des autorités israéliennes compétentes. L'utilisation de ces données par l'OCDE est sans préjudice du statut des hauteurs du Golan, de Jérusalem Est et des colonies de peuplement israéliennes en Cisjordanie aux termes du droit international. 
La passation des marchés publics, qui représentent près de $12 \%$ du produit intérieur brut (PIB) dans les pays de l'Organisation de coopération et de développement économiques (OCDE) et plus de $20 \%$ du PIB en Algérie, est l'une des activités gouvernementales les plus vulnérables aux risques, notamment les risques de corruption et d'atteinte à l'intégrité (OCDE, 2017 $\left.{ }_{[1]}\right)$. Ces risques s'expliquent par les volumes financiers en jeu, la complexité des procédures ainsi que l'interaction et le contact étroit entre les secteurs publics et privés. Préserver l'intégrité et gérer efficacement les différents risques liés aux marchés publics constituent des éléments essentiels pour assurer la bonne gestion des deniers publics, mais également le bon fonctionnement du système dans son ensemble. L'atteinte à l'intégrité dans les marchés publics peut prendre différentes formes, y compris la corruption d'agents de la fonction publique impliqués dans le processus d'attribution des marchés publics, le détournement des fonds, l'influence indue dans l'évaluation des besoins, ou encore la fraude dans l'évaluation des soumissions ou la facturation (OCDE, 2016[2]). La Recommandation du Conseil de l'OCDE sur les marchés publics promeut une utilisation stratégique et globale des achats publics en soulignant douze principes intégrés parmi lesquels l'intégrité constitue un élément clé (encadré 2.1).

\section{Encadré 2.1. Recommandation du Conseil de l'OCDE sur les marchés publics : principe de l'intégrité}

Le Conseil RECOMMANDE que les Adhérents préservent l'intégrité du système de passation des marchés publics à travers des normes générales et des mesures de protection propres à ces marchés. À cet effet, les Adhérents devraient :

i) Exiger un niveau élevé d'intégrité de la part de tous les acteurs du cycle de passation des marchés publics.

ii) Mettre en place des outils d'ordre général visant à assurer l'intégrité au sein du secteur public et les adapter, au besoin, aux risques spécifiques du cycle de passation des marchés publics.

iii) Mettre en place des programmes de formation en matière d'intégrité à destination des agents publics et privés chargés de la passation de marchés, [...].

iv) Formuler des exigences en matière de dispositifs de contrôle interne, de mesures de conformité et programmes anti-corruption pour les fournisseurs, en prévoyant un suivi adapté.

Source : (OCDE, 2015[3]).

L'intégrité ne peut pas être garantie uniquement par les pouvoirs publics, son renforcement nécessite une approche cohérente et globale avec l'implication de toute la société. La Recommandation du Conseil de l'OCDE sur l'intégrité publique souligne la nécessité de se focaliser sur des politiques ad hoc mais également d'adopter une approche globale, basée sur l'évaluation des risques et visant à cultiver une culture de l'intégrité dans la société (OCDE, 2017[4]). Tout en menant des actions pour détecter, enquêter et sanctionner les actes de corruption et autres cas d'atteinte à l'intégrité, il est essentiel dans un premier temps d'entreprendre des actions de prévention plus approfondies afin de résoudre les faiblesses systémiques et institutionnelles qui ouvrent la voie à des actes de corruption ou d'autres pratiques contraires à l'éthique. 


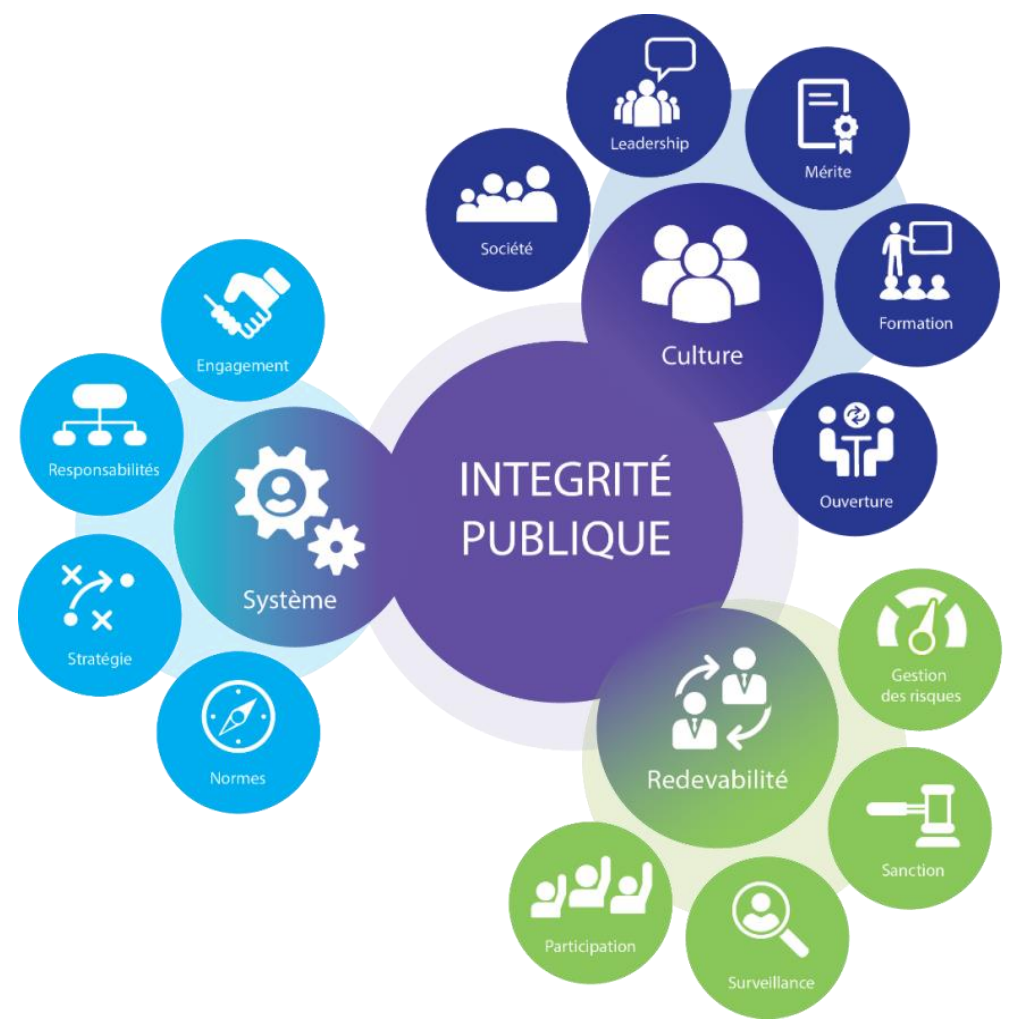

Source : (OCDE, 2017[4]).

Les actes d'atteinte à l'intégrité ont des coûts directs et indirects non négligeables, en particulier dans le domaine des marchés publics. Ils entraînent un gaspillage des ressources publiques, creusent les inégalités économiques et sociales, alimentent le mécontentement et la polarisation politique et réduisent la confiance à l'égard des institutions (OCDE, 2017[4]). Selon la Commission européenne, le coût de la corruption dans les marchés publics peut atteindre 20 à $25 \%$ de la valeur des contrats (Commission Européenne, 2014[5]). Les coûts directs incluent des prix plus importants des biens, services et travaux, de qualité inférieure, ou encore des marchés non exécutés (prestations ou livraisons non réalisées). Les coûts indirects, quant à eux, sont liés à la distorsion de la concurrence, l'accès limité aux marchés, la réduction de l'appétit des investisseurs étrangers et une perte de confiance des citoyens dans leurs gouvernements, ce qui a des conséquences sur la croissance économique (OCDE, 2016[2]).

En Algérie, l'intégrité dans les marchés publics est soulignée comme un élément essentiel dans le cadre réglementaire. Le cadre réglementaire visant à prévenir et lutter contre la corruption, notamment la loi $\mathrm{n}^{\circ}$ 06-01, définit les principes d'intégrité et de lutte contre la corruption applicables aux marchés publics et à la gestion des finances publiques. De plus, le décret présidentiel n¹5-247 de 2015 (DMP) consacre une section entière à la lutte contre la corruption dans les marchés publics et énonce des mécanismes et des outils afin d'y parvenir. Cependant, selon des observateurs internationaux, la corruption est un problème qui affecte l'environnement des affaires en Algérie (graphique 2.2). 
Graphique 2.2.Les facteurs problématiques pour faire des affaires en Algérie (2015-16)

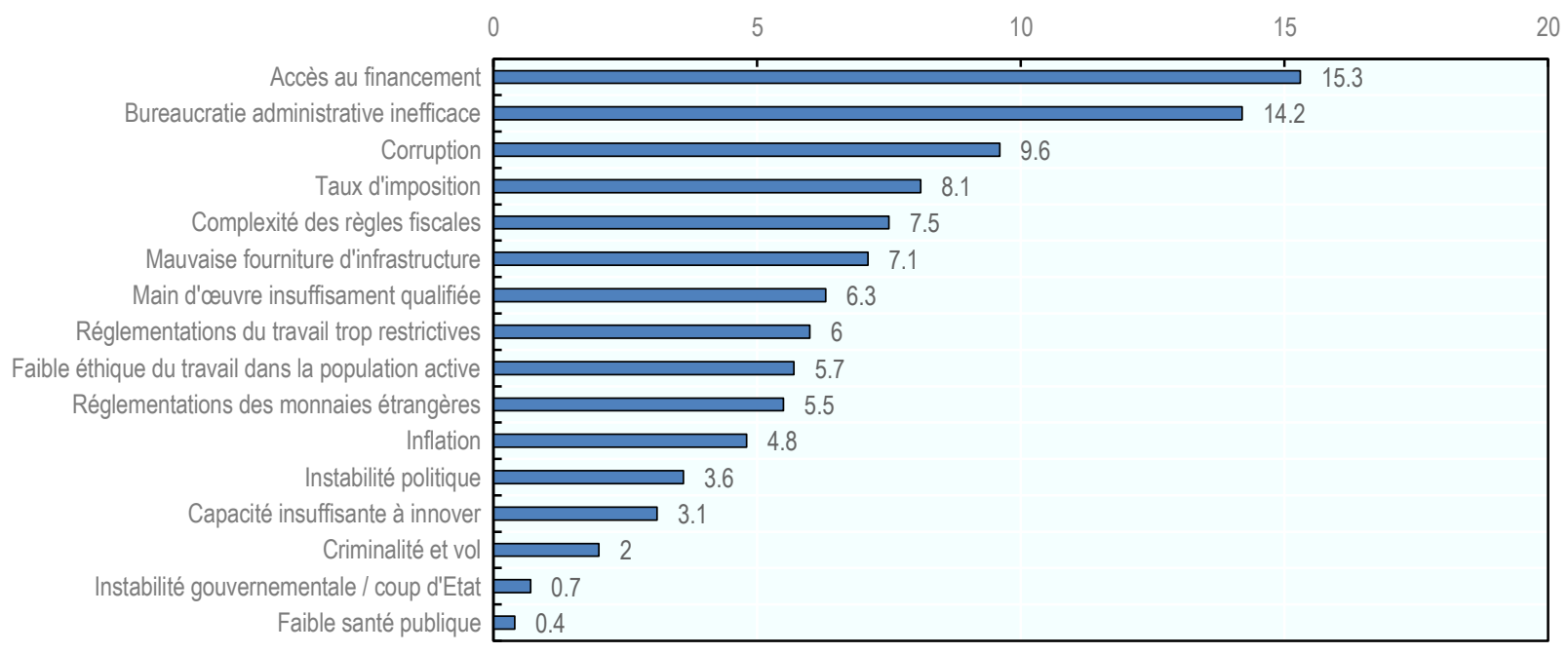

Note : Les répondants ont été invités à identifier cinq facteurs les plus problématiques pour la conduite des affaires dans leurs pays et à les classer de 1 (le plus problématique) à 5 (le moins problématique), par ordre d'importance. La note correspond aux réponses pondérées en fonction de leur importance.

Source : (World Economic Forum, 2015[6]), page 83.

Par ailleurs, en plus de son impact sur l'économie, la corruption influence directement la confiance des citoyens en leurs gouvernements. Selon les indices publiés par Transparency International (non reconnus par le gouvernement algérien), le niveau de corruption perçue en Algérie et dans des pays d'Afrique du Nord est supérieur à celui observé dans les pays membres de l'OCDE. Le graphique 2.3 présente l'indice de perception de la corruption en 2017 en Algérie, dans les pays de l'OCDE et dans certains pays d'Afrique du Nord.

\section{Graphique 2.3. Indice de perception de la corruption (2017)}

Niveaux perçus de corruption au sein du secteur public sur la base d'appréciations d'experts des secteurs privés et publics

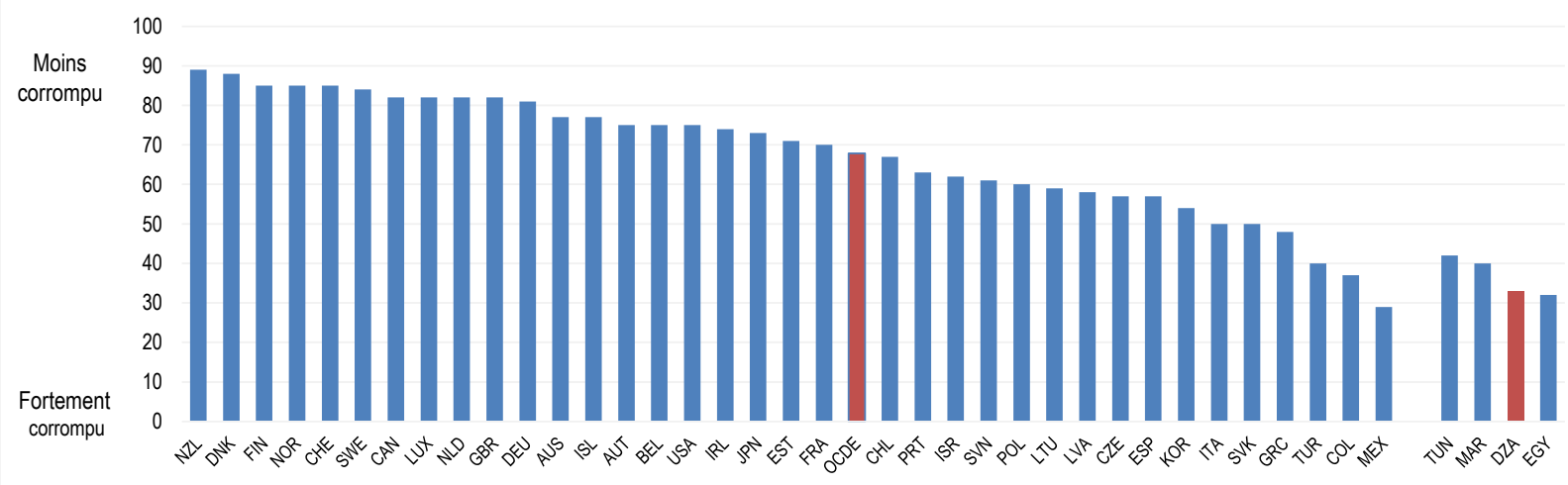

Source : (Transparency International, 2018[7]).

Ce chapitre analyse le système d'intégrité des marchés publics en Algérie et fournit des recommandations conformes aux bonnes pratiques internationales. 


\subsection{Renforcer le système d'intégrité dans les marchés publics}

Afin de mettre en place un système d'intégrité publique cohérent et global, la Recommandation du Conseil de l'OCDE sur l'intégrité publique met particulièrement l'accent sur les nécessités suivantes : faire preuve d'engagement, aux plus hauts niveaux politiques et de gestion du secteur public; expliciter les responsabilités institutionnelles à l'échelle du secteur public; élaborer une approche stratégique concernant le secteur public fondée sur des données factuelles et destinée à atténuer les risques en matière d'intégrité publique ; et fixer des normes de conduites strictes applicables aux agents publics (OCDE, 2017 $[4])$. Ces éléments sont également applicables pour préserver l'intégrité dans les marchés publics. Dans de nombreux pays de l'OCDE, le cadre réglementaire et institutionnel sur l'intégrité reflète ces éléments, et des mesures et des outils spécifiques sont prévus pour les marchés publics.

\subsubsection{Développer une stratégie nationale et clarifier les standards d'intégrité dans les marchés publics}

De nombreux pays, essentiellement parmi les économies émergentes, ont développé des stratégies de lutte contre la corruption afin de mettre en œuvre des politiques multidimensionnelles coordonnées et effectives (UNDP, 2014[8]). Pour obtenir des résultats concrets, l'OCDE recommande de développer une approche stratégique :i) en fixant des objectifs et des priorités stratégiques pour le système d'intégrité publique qui reposent sur une approche des violations des normes d'intégrité publique axée sur les risques, et tenant compte des facteurs qui jouent un rôle dans l'efficacité des politiques d'intégrité publique ; et ii) en élaborant des éléments de référence et des indicateurs, et en recueillant des données fiables et pertinentes sur le niveau de mise en œuvre, de performance et d'efficacité générale du système d'intégrité publique (OCDE, 2017[4]).

En Algérie, la loi n 06-01 du 20 février 2006 a prévu la création de l'Organe national de prévention et de lutte contre la corruption (ONPLC) afin de mettre en œuvre la stratégie nationale en matière de lutte contre la corruption. Suite à sa mise en place en 2011, cinq ans après la promulgation de la loi $n^{\circ} 06-01$, l'Algérie a établi un plan d'action du gouvernement pour 2012-14 qui incluait des mesures pour la lutte contre la corruption. Bien que ce plan d'action ne soit pas disponible en ligne et qu'il n'ait pas été transmis à l'équipe OCDE, un rapport de l'Office des Nations Unies contre la drogue et le crime (UNODC) mentionne qu'il comprend des dispositions relatives aux marchés publics, y compris par exemple l'amélioration de la transparence dans le traitement des marchés publics et l'introduction de l'obligation de déclaration de probité dans les procédures de soumission aux marchés publics (UNODC, 2015[9]). Depuis ce plan d'action, aucune stratégie ou plan d'action n'ont été développés ou mis à jour. Cependant, d'après les entrevues réalisées lors des missions exploratoires, l'ONPLC développe actuellement une stratégie nationale pour préserver l'intégrité et lutter contre la corruption.

\section{Développer et mettre en œuvre une stratégie pour préserver l'intégrité dans les marchés publics}

L'Algérie a montré un engagement fort par la signature et la ratification de plusieurs conventions internationales dans le domaine de la lutte contre la corruption et la protection de l'intégrité. L'Algérie est l'un des premiers pays à avoir adopté et ratifié la Convention des Nations Unies contre la corruption (CNUCC), et la Convention de l'Union africaine sur la prévention et la lutte contre la corruption. Ces deux conventions ont été ratifiées respectivement par les décrets présidentiels $n^{\circ}$ 04-128 du 19 avril 2004 et $\mathrm{n}^{\circ}$ 06-137 du 10 avril 2006.

Le cadre juridique de l'Algérie reconnaît les marchés publics comme un domaine à haut risque. L'article 9 de la CNUCC, dédié à la passation des marchés publics, stipule que ceux-ci doivent être fondés " sur la transparence, la concurrence et des critères objectifs pour la prise des décisions et qui soient efficaces, entre autres, pour prévenir la corruption ». Conformément à la CNUCC, la loi n 06-01 du 20 février 2006, 
modifiée et complétée, relative à la prévention et à la lutte contre la corruption, dispose d'un article dédié aux marchés publics qui énonce les principes de la passation des marchés publics. Elle détaille également les mesures et les sanctions applicables aux actes de corruption et aux avantages injustifiés dans les marchés publics, ainsi que les moyens d'enquête qui peuvent être utilisés. La loi définit par ailleurs les actes de corruption dans le secteur public et énonce des sanctions liées aux infractions applicables aux agents publics impliqués dans les procédures de passation des marchés publics et au secteur privé. L'application de la loi $n^{\circ}$ 06-01 est détaillée par d'autres textes réglementaires, notamment l'ordonnance $n^{\circ}$ 07-01 du 1er mars 2007 relative aux incompatibilités et obligations particulières attachées à certains emplois et fonctions; le décret présidentiel $n^{\circ}$ 06-414 du 22 novembre 2006 , fixant le modèle de la déclaration de patrimoine ; et le décret présidentiel n 06-415 du 22 novembre 2006, fixant les modalités de déclaration de patrimoine des agents publics.

Bien que les politiques de lutte contre la corruption en Algérie reconnaissent les marchés publics comme un domaine à haut risque, elles se focalisent principalement sur les mesures pour détecter et sanctionner les atteintes à l'intégrité, notamment la corruption, sans établir de vision nationale avec des objectifs à atteindre pour la promotion d'une culture d'intégrité fondée sur les valeurs et des normes d'intégrité dans le secteur public - y compris dans les marchés publics. Cette approche fondée sur les sanctions peut empêcher des actes de corruption en créant un environnement de crainte (voir la section 2.3.3 pour les sanctions). Cependant, les expériences menées dans de nombreux pays ont montré que les approches basées sur les sanctions doivent être accompagnées d'une approche globale de promotion des valeurs d'intégrité, de transparence et de responsabilité afin de permettre d'établir une vision nationale porteuse d'incitations positives à se conformer aux valeurs d'intégrité pour les agents publics et le secteur privé (Conseil de l'Europe, 2013 [10]). L'Algérie gagnerait donc à développer une stratégie qui définisse une vision nationale avec des objectifs clairs, basés sur les normes d'intégrité, en prenant en compte les domaines à haut risque tels que les marchés publics. Par ailleurs, ces stratégies devraient refléter les engagements internationaux du pays en la matière.

Les marchés publics étant un domaine à haut risque, lors du développement de stratégies de lutte contre la corruption, les pays adoptent en général deux approches :i) une stratégie spécifique aux marchés publics qui découle d'une stratégie nationale plus globale ; ou ii) une stratégie nationale qui comporte des dispositions spécifiques aux marchés publics (OCDE, 2019[11]). L'encadré 2.2 présente des exemples de ces deux approches au Royaume-Uni (pour une stratégie nationale avec des dispositions spécifiques aux marchés publics) et en Grèce (pour une stratégie spécifique aux marchés publics). 


\section{Encadré 2.2. Les deux approches de stratégie de lutte contre la corruption dans les marchés} publics, exemples du Royaume-Uni et de la Grèce

\section{Stratégie nationale de lutte contre la corruption du Royaume-Uni pour la période 2017-22}

La stratégie de lutte contre la corruption du Royaume-Uni permet de définir une vision pour un avenir plus sûr et prospère, basé sur une action concertée du pays pour lutter contre la corruption à long terme. Cette stratégie s'appuie sur le « plan anti-corruption » du Royaume-Uni de 2014 et sur le travail déjà entrepris au sein du gouvernement, notamment par le biais de la stratégie de sécurité nationale, de la stratégie sur les délits graves et crimes organisés, du plan d'action contre le blanchiment d'argent et le financement de la lutte contre le terrorisme, et de la stratégie de lutte contre la fraude et la corruption au niveau local.

La stratégie reconnaît que la corruption menace la sécurité et la prospérité nationale au Royaume-Uni et à l'étranger, et que sans mesures concrètes cela peut éroder la confiance des citoyens dans les institutions nationales et internationales. Elle identifie cinq priorités, dont une directement liée aux marchés publics (« Réduire la corruption dans les marchés publics et les subventions »), qui comprend trois objectifs principaux :

1. Renforcer la transparence dans les marchés publics et améliorer l'identification et la réduction des risques.

2. Consolider la sensibilisation et les capacités au sein des entités adjudicatrices.

3. Améliorer la confiance envers une gestion contractuelle légitime et efficace.

\section{Stratégie nationale de lutte contre la corruption dans les marchés publics de la Grèce pour la période 2016-20}

Le gouvernement grec a adopté en juin 2017 la stratégie nationale pour les marchés publics qui inclut un volet relatif à la lutte contre la corruption et à la promotion de l'intégrité. Ce volet est conforme à la stratégie nationale de lutte contre la corruption. Par ailleurs, la stratégie nationale pour les marchés publics prévoit des dispositions spécifiques pour les marchés publics du domaine de la santé et des travaux publics, qui ont été évalués comme des domaines à haut risque.

Sources: (HM Government, 2017[12]) ; (OCDE, s.d.[13]).

La lutte contre la corruption nécessite une approche globale et concertée, pour cela, le gouvernement algérien pourrait adopter l'une de ces deux approches: i) développer une stratégie spécifique pour préserver l'intégrité dans les marchés publics qui découlerait d'une stratégie nationale ; ou ii) développer une stratégie nationale qui prend en compte les spécificités des risques inhérents aux marchés publics. Dans les deux cas, la stratégie devrait mettre l'accent sur les défis actuels et les actions spécifiques à mener.

\section{Désigner un chef de file et adopter une approche collaborative pour le développement de la stratégie de lutte contre la corruption}

Le développement de la stratégie pour préserver l'intégrité dans les marchés publics nécessite la participation de différentes parties prenantes afin d'assurer la mise en place effective de mesures relatives aux compétences fondamentales de chacune des parties prenantes. Par ailleurs, leurs connaissances et expériences sont des contributions pertinentes pour la définition d'une vision nationale d'un système 
d'intégrité. La participation des différentes parties prenantes contribue donc à assurer une compréhension commune des priorités et une appropriation de la stratégie (OCDE, 2019[14]).

En Algérie, selon l'approche choisie par le pays (développer une stratégie spécifique pour l'intégrité dans les marchés publics qui découlerait d'une stratégie nationale ou développer une stratégie nationale prenant en compte les spécificités des marchés publics), l'entité « cheffe de file » peut être différente. En effet dans le premier cas, le chef de file pour la stratégie relative aux marchés public pourrait être l'Autorité de régulation des marchés publics et des délégations de service public (ARMP) en raison de ses attributions réglementaires et de sa connaissance des spécificités du domaine. Dans le second cas, le chef de file devrait être l'ONPLC, conformément à son mandat, qui est de proposer une politique globale de prévention de la corruption. Cependant, l'ARMP n'a toujours pas été mise en place plus de trois ans après l'entrée en vigueur du DMP. L'Algérie devrait donc mettre en place l'ARMP afin de renforcer la stratégie et la politique de lutte contre la corruption dans les marchés publics en coordination avec les autres entités en charge de ces politiques.

Divers acteurs doivent contribuer au développement et à la mise en place de la stratégie (graphique 2.4).Tout d'abord, les autorités impliquées dans la conception et la mise en œuvre de la politique nationale d'intégrité et de lutte contre la corruption, en particulier l'ONPLC et l'Office central de répression de la corruption. Un projet de loi visant à dissoudre l'OCRC a été adopté par le Conseil des Ministres le 27 décembre 2018. L'OCRC serait remplacé par un pôle pénal financier créé à la Cour de justice d'Alger et dont la compétence s'étend au niveau national (ALG24, s.d.[15]). Par ailleurs, la Cellule de traitement du renseignement financier (CTRF), chargée de lutter contre le financement du terrorisme et le blanchiment d'argent, peut également jouer un rôle important.

L'implication du ministère de la Justice est également nécessaire afin de prévoir une définition pénale des cas d'atteinte à l'intégrité qu'il devra instruire et des éventuelles sanctions applicables. Le ministère de la Fonction publique devra également prendre part au développement de la stratégie car celle-ci peut avoir des conséquences sur des éléments relevant de sa compétence, comme par exemple les sanctions administratives et les actions de sensibilisation et de renforcement de capacité des agents publics sur les problématiques d'atteinte à l'intégrité. Enfin, le volet formations nécessite l'implication du ministère de l'Enseignement supérieur et des Instituts de formation.

Des agents chargés de la passation des marchés publics peuvent intervenir en tant qu'experts du système de marché public pour mettre en avant les procédures exposées aux plus grands risques afin que la stratégie prévoie des mesures permettant aux agents exposés de s'en prémunir.

Les organes de contrôle interne et externe contribueront au développement de la stratégie en soulignant les failles récurrentes et constatées et en veillant à leur correction. La société civile devra également être mise à contribution, conformément à l'article 15 de la loi 06-01 du 20 février 2006, qui stipule en effet que la société civile doit prendre part à la transparence des processus de décision et la gestion des affaires publiques, être sensibilisée à la corruption (notamment grâce à des programmes d'enseignement et d'éducation), et que les médias et le public doivent avoir accès aux informations concernant la corruption.

Enfin, le secteur privé doit être mis à contribution, notamment afin de s'assurer que la stratégie développée réponde aux besoins des fournisseurs potentiels en termes de sensibilisation à l'intégrité, et que les attentes et les valeurs du secteur public en la matière soient bien comprises par le secteur économique.

En conclusion, l'Algérie gagnerait à impliquer l'ensemble des parties prenantes pour le développement de la stratégie d'amélioration de l'intégrité et de lutte contre la corruption, y compris dans le domaine des marchés publics. 
Graphique 2.4.Acteurs à impliquer dans le développement d'une stratégie de lutte contre la corruption dans le domaine des marchés publics

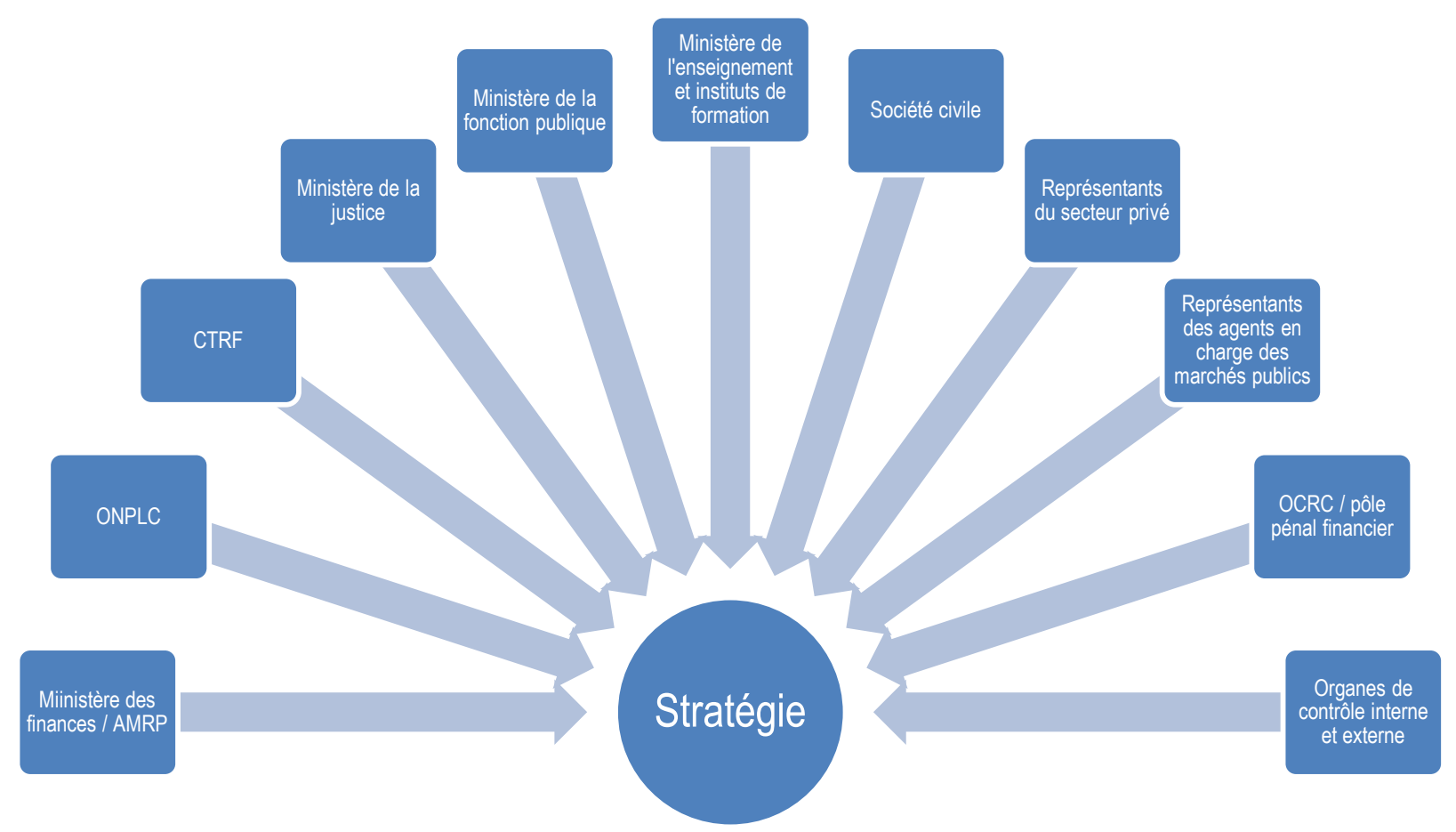

\subsubsection{Clarifier les rôles institutionnels des autorités impliquées dans le système d'intégrité des marchés publics et accroître la transparence de leurs activités}

La lutte contre la corruption ne consiste pas seulement à diagnostiquer des problèmes et à appliquer des solutions individuelles. Elle nécessite une approche globale du secteur public et de toute la société. À cette fin, toutes les entités publiques doivent être dotées de responsabilités claires et de capacités appropriées (OCDE, 2017[16]).

Les politiques de lutte contre la corruption ciblent notamment les groupes d'intérêt qui tentent d'influencer les processus de prise de décision. Les institutions en charge de la lutte contre la corruption doivent donc être protégées de toute influence politique indue, en particulier lors de changements de gouvernement. Par ailleurs, les politiques de lutte contre la corruption et de protection de l'intégrité, en particulier les mesures préventives, ont généralement besoin de temps pour que leur impact se révèle : cela renforce la nécessité de garantir leur indépendance et la continuité de leurs activités (OCDE, 2017[16]).

\section{Améliorer la transparence et la reddition des comptes des activités de l'ONPLC}

L'ONPLC a été mis en place en 2011, cinq ans après l'adoption de la loi qui prévoyait sa création. L'ONPLC est placé auprès du président de la République et dispose de l'autonomie financière. II est chargé principalement de : recueillir les déclarations de patrimoine et analyser les informations et les statistiques relatives aux déclarations ; mettre en place des actions de formation et de sensibilisation ; et de préparer un rapport annuel à destination du président de la République.

Pour renforcer son indépendance et son efficacité, l'ONPLC est doté d'un conseil de veille et d'évaluation dont les premiers membres ont été désignés en 2011. II est composé du Président de l'ONPLC, nommé par le président de la République, et de six membres nommés par décret présidentiel pour une durée de cinq ans renouvelable une seule fois, choisis parmi les personnalités nationales indépendantes 
représentatives de la société civile, connues pour leur intégrité et leurs compétences (ONPLC, s.d.[17]). Le conseil donne son avis sur le fonctionnement et les activités de l'ONPLC - y compris le programme d'actions de l'organe et les conditions et modalités de son application; les rapports, avis et recommandations de l'organe ; ainsi que le bilan annuel et le budget de l'organe.

Les rapports, avis et recommandations de l'ONPLC peuvent contenir des informations relatives aux marchés publics et à la bonne gestion des deniers publics. Cependant, les études et rapports de l'ONPLC ne sont pas publics, ce qui n'est pas conforme aux bonnes pratiques internationales. En effet, il est du devoir des gouvernements d'informer la société civile et les différentes parties prenantes des actions et de l'efficacité du système d'intégrité en partageant des informations et données statistiques sur ce sujet, y compris dans le domaine des marchés publics (OCDE, 2017[4]). Par ailleurs, ces rapports et études ne sont pas partagées avec certaines parties prenantes du système, telles que le ministère des Finances (pour les marchés publics mais également pour d'autres sujets, tels que les douanes). Par exemple, l'Agence française anticorruption, qui a pour mission d'élaborer des recommandations dans la mise en œuvre de procédures internes de prévention et de détection des faits de corruption, de trafic d'influence, de concussion, de prise illégale d'intérêt, de détournement de fonds publics et de favoritisme, rend ses recommandations publiques et publie un rapport annuel d'activité (AFA (Agence Française Anticorruption), 2018[18]). L'Algérie gagnerait donc à publier les rapports et les recommandations de l'ONPLC, ainsi que les avis du conseil de veille et d'évaluation sur le site web de l'ONPLC, mais également à les partager avec d'autres parties prenantes responsables de domaines à haut risque de corruption, telles que le ministère des Finances pour les marchés publics.

\section{S’assurer de la mise en place effective des activités d'investigation des infractions de corruptions et renforcer la coordination dans le domaine de la lutte contre la corruption dans les marchés publics}

L'OCRC est un acteur principal dans la détection des infractions de corruption, il avait également pour mandat de mener des recherches et des enquêtes. L'OCRC a été créé par le décret présidentiel du 8 décembre 2011 portant sur sa composition, organisation et modalités de fonctionnement, cinq ans après l'adoption de la loi n 06-01, qui prévoyait sa création ; il n'a été officiellement mis en place qu'en 2013.

L'OCRC est composé d'officiers de police judiciaire chargés principalement de :i) collecter, centraliser et exploiter toute information permettant de détecter et de lutter contre les actes de corruption ; ii) rassembler les preuves, enquêter sur des faits de corruption et en déférer les auteurs devant la juridiction compétente ; iii) développer la collaboration et l'entraide avec les organismes de lutte contre la corruption et l'échange d'informations à l'occasion des enquêtes en cours; et iv) proposer aux autorités compétentes toute mesure de nature à préserver le bon déroulement des investigations dont il a la charge. D'après les missions exploratoires de l'OCDE, parmi une dizaine d'enquêtes menées par l'OCRC entre juillet 2017 et juillet 2018, aucune n'était relative aux marchés publics. Alors que le système de passation des marchés publics représente en général un des domaines à hauts risques de corruption, cette situation pose la question de l'efficacité et de la transparence du système.

Comme évoqué à la section 2.1.1, un projet de loi visant à dissoudre l'OCRC a été adopté par le Conseil des Ministres le 27 décembre 2018 . L'OCRC devrait être remplacé par un pôle pénal financier, qui sera créé à la Cour de justice d'Alger et dont la compétence s'étendra au niveau national (ALG24, s.d.[15]). La création de ce pôle est prévue dans un projet de loi relatif à la lutte contre la corruption présenté le 4 février 2019 par le ministère de la Justice à l'Assemblée populaire nationale. Ce projet de loi a pour but « d'adapter la loi n 06-01 du 20 février 2006 aux dispositions de la Constitution révisée en 2016 ». Les prérogatives de ce pôle financier concernent « la recherche, l'enquête, le suivi et l'investigation sur les crimes financiers complexes, les crimes liés à la corruption, à la fraude et à l'évasion fiscale, au financement des associations et les crimes liés au change et aux établissements financiers et bancaires ». Les compétences du pôle pénal financier et de l'OCRC ne semblent donc pas identiques (Liberté Algérie, 
s.d.[19]).En cas de dissolution définitive de l'OCRC et de la création du pôle pénal financier, le gouvernement algérien devrait s'assurer que les activités de ce pôle reprennent au minimum celles de I'OCRC et qu'elles soient transparentes et efficaces afin d'assurer une lutte effective contre la corruption, y compris dans le domaine des marchés publics.

Les discussions menées lors des missions exploratoires de l'OCDE ont démontré que le système souffrait du manque de coordination entre les différentes entités chargées de la détection et de la répression des cas d'atteinte à l'intégrité, mais également avec celles chargées des marchés publics. Par ailleurs, lorsque l'OCRC constatait des irrégularités dans ses enquêtes, il transmettait le dossier au ministère de la Justice afin d'y donner suite. Cependant, il n'existe pas de communication entre les deux autorités quant à la suite donnée à l'instruction des dossiers. L'Algérie pourrait renforcer sa contribution au système de lutte contre la corruption en rendant publiques les informations sur les activités des autorités chargées de la lutte contre la corruption, tout en assurant une confidentialité afin de ne pas entraver l'instruction des dossiers en coordination avec le ministère de la Justice. Par ailleurs, dans le cadre des marchés publics, les entités en charge de la détection des infractions de corruption devront également se coordonner davantage avec les autres autorités impliquées dans le système de passation et de contrôle des marchés publics, y compris le ministère des Finances, la Cour des comptes, l'Inspection générale des finances et la CTRF afin de mieux détecter les infractions de corruption dans les marchés publics.

\subsection{Développer et entretenir une culture d'intégrité dans les marchés publics}

Créer une culture d'intégrité dans les marchés publics nécessite la définition de valeurs communes auxquelles tous les agents impliqués doivent adhérer, et l'élaboration de normes de conduite concrètes que les agents doivent appliquer dans leur travail quotidien. Dans le contexte des marchés publics, l'intégrité implique notamment :

- que des normes d'intégrité applicables aux agents impliqués dans les marchés publics soient promulguées par le gouvernement,

- qu'une unité gouvernementale, une direction ou un bureau dédié soit consacré au développement, à la mise à jour et à la diffusion de normes d'intégrité reflétées dans un code de conduite du gouvernement, et que cette entité puisse donner des conseils, des orientations et des exemples pratiques pour assurer sa mise en œuvre,

- que des normes de conduite spécifiques pour les agents publics travaillant dans les marchés publics soient adoptées afin d'atténuer les risques spécifiques liés à la complexité et aux caractéristiques du processus de passation des marchés publics,

- que les conflits d'intérêts soient correctement gérés pour que l'implication des agents publics dans le cycle de passation des marchés publics n'influence pas indûment l'exercice des fonctions et des responsabilités de ces agents,

- que le gouvernement établisse des règles et des procédures pour protéger les lanceurs d'alertes.

La compréhension commune de l'intégrité dans les marchés publics par toutes les parties prenantes est cruciale afin de développer et nourrir une culture d'intégrité (OCDE, 2018[20]).

\subsubsection{Développer et mettre en place un code de conduite pour les agents en charge de marchés publics}

Reconnaissant l'importance de l'intégrité en tant que responsabilité individuelle mais également des institutions publiques, de nombreux pays ont développé des codes de conduite afin d'établir les normes et les exigences en matière de bonne conduite des fonctionnaires et agents publics. La plupart des pays possèdent un code de conduite à destination de l'ensemble des agents publics, et certains ont développé 
un code de conduite spécifique pour les agents chargés des marchés publics en reconnaissant les risques spécifiques de ce domaine. Celui-ci établit non seulement les exigences minimales mais promeut également les intérêts publics et encourage des normes de conduite élevées, une bonne gouvernance et le respect des valeurs de la fonction publique (OCDE, 2017[16]). L'encadré 2.3 propose des conseils pour renforcer la qualité et la pertinence d'un code de conduite.

\section{Encadré 2.3. Guide pour la rédaction d'un code de conduite}

Les conseils suivants peuvent renforcer la qualité et la pertinence d'un code qui adopte un modèle composé d'un nombre restreint de valeurs fondamentales, définies puis spécifiées dans des règles, accompagnées d'exemples :

- Clair : rendre le texte aussi clair et lisible que possible ; le code doit être clair pour tous les agents qui sont censés l'appliquer.

- Simple : rendre le texte aussi simple que possible. L'intégrité est un sujet compliqué et cela ne doit pas être négligé. Cependant, il n'y a aucune raison de rendre les explications plus compliquées que nécessaire.

- Concret : éviter les généralisations vaines. Les dispositions vagues ne sont pas toujours évitables, en particulier dans les codes d'éthique qui sont fondés sur des valeurs. Néanmoins, il est important de les définir d'une manière concrète (par exemple, en les précisant dans des règles et conseils spécifiques ou en les illustrant avec des exemples).

- Structuré : s'assurer que le code est construit logiquement et centré sur des valeurs fondamentales qui ne se chevauchent pas. Si les valeurs sont exclusives, il serait plus simple d'identifier les conflits qui existent entre elles. Ces conflits sont typiques des dilemmes éthiques et un code avec des valeurs clairement définies devient un outil très utile pour traiter les dilemmes éthiques ou donner des sessions de formation relatives au dilemme.

- Cohérent : utiliser les concepts de manière parcimonieuse et cohérente. Ce n'est pas une bonne pratique d'utiliser des termes différents pour le même concept dans le même code (ou dans des documents différents au sein de la même organisation). II faut choisir le terme le plus approprié et l'utiliser de manière cohérente dans les différents documents. Cela augmentera considérablement les chances d'utilisation du même langage par l'ensemble des agents, permettant ainsi au code et aux documents associés de devenir des outils utiles pour la formation et les échanges quotidiens.

- Lié : inclure suffisamment de références croisées dans le code avec d'autres documents, directives et codes, afin que les agents puissent trouver plus de détails sur les thèmes spécifiques.

- Pertinent : le code devrait aller au-delà de l'évidence et se concentrer sur les problèmes nécessitant une orientation. Le code sera plus pertinent si des analyses de risque et de dilemme sont utilisées dans sa préparation.

Source : (OCDE, 2018[21]).

En Algérie, l'article 88 du DMP prévoit un code d'éthique et de déontologie des agents publics intervenant dans le contrôle, la passation et l'exécution des marchés publics et des délégations de service public. Cette bonne pratique inclut non seulement les acheteurs publics, mais aussi l'ensemble des agents publics impliqués dans les marchés publics. 
Un projet de code d'éthique et de déontologie des agents publics chargés de la passation des marchés publics a été préparé par l'ONPLC à l'intention des services du ministère des Finances et à leur demande suite à l'adoption du décret présidentiel précédent sur les marchés publics, datant de 2010. Cependant, bien que ce projet de code soit publié sur le site de l'ONPLC, il n'a jamais été adopté et donc mis en application (ONPLC, s.d.[22]). Par ailleurs, les discussions menées avec l'ONPLC lors des missions exploratoires de l'équipe de l'OCDE ont confirmé que l'ONPLC élabore un code de conduite, en coordination avec la direction générale de la fonction publique. Afin de se conformer à son cadre réglementaire et d'assurer des normes d'intégrité communes dans le secteur public, l'Algérie devrait développer et adopter le code d'éthique et de déontologie pour les agents publics impliqués dans les marchés publics, en prenant en compte les risques spécifiques à ce domaine. Ce code de d'éthique pourra être aligné avec le code de conduite des agents publics en cours d'élaboration par l'ONPLC et la direction générale de la fonction publique afin d'établir des valeurs et normes commune d'intégrité. De plus, afin de renforcer la pertinence et l'impact de ce code, l'Algérie pourrait consulter les agents publics impliqués dans les marchés publics pour assurer un "sentiment d'appropriation ", mieux comprendre les dilemmes éthiques et les risques d'atteinte à l'intégrité dans les marchés publics.

L'Algérie rencontre des difficultés d'identification des agents impliqués dans le cycle de passation des marchés publics (voir chapitre 6). Elle doit donc clairement identifier ces agents et leur responsabilité dans le processus : en effet, en plus des agents en charge de la rédaction des cahiers des charges et des documents administratifs et juridiques, ce code doit s'appliquer aux membres des commissions des marchés, des commissions d'ouverture des plis et d'évaluation des offres, ainsi qu'aux agents impliqués dans le suivi d'exécution des contrats publics. Le champ d'application de ce code peut même inclure le secteur privé, ce qui permettrait le partage de valeurs communes. C'est pourquoi des pays comme le Canada ont développé un code de conduite unique pour les agents publics en charge des marchés publics et les fournisseurs(voir encadré 2.4). En tout état de cause, l'Algérie devrait s'assurer de communiquer les valeurs du secteur public au secteur privé afin que ce dernier soit conscient des attentes du secteur public.

\section{Encadré 2.4. Code de conduite de marchés publics au Canada}

Le Code de conduite pour l'approvisionnement au Canada fournit à toutes les personnes qui participent au processus d'approvisionnement - fonctionnaires ou fournisseurs - un énoncé clair des attentes mutuelles de façon à ce que tous les participants aient une connaissance de base commune.

Le Code reflète la politique du gouvernement du Canada et est encadré par les principes établis dans la Loi sur la gestion des finances publiques et la Loi fédérale sur la responsabilité. II regroupe les mesures relatives aux conflits d'intérêt et à la lutte contre la corruption du gouvernement fédéral, ainsi que d'autres exigences de lois et de politiques concernant spécifiquement l'approvisionnement. Ce code résume la loi existante en offrant un point de référence unique pour les responsabilités et les obligations clés des fonctionnaires et fournisseurs. En outre, il décrit le mécanisme de recours des fournisseurs et les garanties procédurales.

Source : (Services publics et Approvisionnement Canada (SPAC), 2018[23]).

Par ailleurs, le DMP prévoit l'obligation de signer la déclaration de probité par tous les soumissionnaires. Cette déclaration prévoit que le soumissionnaire : i) s'engage à ne recourir à aucun acte ou manœuvre dans le but de faciliter ou de privilégier le traitement de son offre au détriment de la concurrence loyale ;et ii) s'engage à ne pas s'adonner à des actes ou à des manœuvres tendant à promettre d'offrir ou d'accorder à un agent public, directement ou indirectement, soit pour lui-même ou pour une autre entité, une rémunération ou un avantage de quelque nature que ce soit, à l'occasion de la préparation, de la négociation, de la passation, de l'exécution ou du contrôle d'un marché public ou d'un avenant. Cependant aucune statistique ou information n'est disponible quant à l'efficacité de ce mécanisme. L'Algérie devrait 
donc mener des actions de sensibilisation afin que ces déclarations ne soient pas remplies comme un document administratif mais comme un document juridique démontrant l'engagement du secteur privé à respecter les valeurs du secteur public. Par ailleurs, la Commission d'ouverture et d'évaluation des plis devra s'assurer que ces fournisseurs ne sont pas dans la liste des fournisseurs exclus temporairement ou définitivement.

\subsubsection{Une gestion efficace des conflits d'intérêts dans le système de passation des marchés publics}

L'environnement du secteur public changeant rapidement, les conflits d'intérêts seront toujours une source de préoccupation, en particulier dans le domaine des marchés publics. Une politique moderne de gestion des conflits d'intérêts devrait donc s'efforcer de trouver un équilibre en déterminant les risques qui menacent l'intégrité des organismes publics et des agents publics, en interdisant les formes de conflit d'intérêts inacceptables, en gérant convenablement les situations de conflit, en sensibilisant les organismes publics et les agents publics à l'incidence de ce type de conflits et en veillant à ce que des procédures efficaces soient mises en place pour identifier, divulguer, gérer et favoriser un règlement adéquat de ces situations de conflit d'intérêts. (OCDE, 2003[24])

L'identification et la gestion adéquate des conflits d'intérêts figurent parmi les premières étapes du renforcement de l'intégrité et de la transparence du secteur public. Les pays membres de l'OCDE disposent de politiques, règles et procédures pour assurer la gestion efficace des conflits d'intérêts dans le secteur public. Les interactions étroites entre le secteur public et le secteur privé dans la passation des marchés publics, et les enjeux financiers importants impliqués dans ces marchés engendrent des risques de conflits d'intérêts (OCDE, 2017[16] $)$, c'est pourquoi près de la moitié des pays de l'OCDE ont élaboré des politiques ou des règles spécifiques à la gestion des conflits d'intérêts dans les marchés publics. (OCDE, 2016[2])

\section{Définir et déclarer les conflits d'intérêts dans le cadre des marchés publics}

Le conflit d'intérêts ne représente pas un acte de corruption et les agents publics ont des intérêts légitimes liés à leur capacité en tant que citoyen. Une définition claire et réaliste des circonstances et des relations qui peuvent conduire à un conflit d'intérêts est indispensable pour un système d'intégrité efficace. Un conflit d'intérêts peut être définit comme " un conflit entre la mission publique et les intérêts privés d'un agent public, dans lequel l'agent public a des intérêts privés qui pourraient influer indûment sur l'exercice de ses fonctions et responsabilités ». (OCDE, 2006[25])

Les agents publics impliqués dans les marchés publics peuvent voir leurs intérêts privés en conflit avec leur mission tout au long du cycle de passation des marchés publics. Le conflit d'intérêts, qu'il soit réel, potentiel ou apparent, doit être clairement expliqué aux agents, comme cela est fait en Nouvelle-Zélande (encadré 2.5). En plus de la définition du concept, il est également nécessaire de fournir des indications claires sur comment gérer ces conflits d'intérêts. (OCDE, 2006 ${ }_{[25]}$ ) 


\section{Encadré 2.5. La définition des conflits d'intérêts en Nouvelle-Zélande}

Un conflit d'intérêts peut survenir d'une relation, d'une activité ou de points de vue personnels fermes.

Un employé est en conflit d'intérêts si, dans le cadre de ses fonctions, il doit travailler avec :

- un parent ou un ami personnel proche,

- une organisation, un club, une société ou une association dont il est membre,

- une personne qui est son chef de communauté ou d'église,

- une personne ou une organisation : i) auprès de laquelle il a une obligation professionnelle ou légale ; ii) auprès de laquelle il a un intérêt commercial ou possède une propriété ; iii) à qui il doit de l'argent ; et iv) pour qui il a déjà travaillé ou travaille actuellement (emploi secondaire).

Un employé d'agence peut également avoir un conflit d'intérêts s'il reçoit de la part d'une personne ou d'une organisation qui pourrait bénéficier de ses décisions :

- un cadeau,

- une invitation pour un déjeuner, un dîner ou un événement sportif,

- un voyage ou un hébergement gratuit ou subventionné,

- tout autre type d'avantage, y compris de l'argent.

Un conflit peut également survenir si une personne a des opinions personnelles fortes sur un problème que son agence envisage, par exemple des opinions politiques ou des convictions religieuses.

Source : (New Zealand Government Procurement and Property, s.d.[26]).

En Algérie, le cadre réglementaire régissant la lutte contre la corruption inclut des dispositions relatives à l'identification et à la gestion de conflit d'intérêts. L'article 34 de la Loi relative à la prévention et à la lutte contre la corruption catégorise le conflit d'intérêts comme un crime. Cependant, si cet article énonce que le non-respect par l'agent public des principes des procédures applicables en matière de marchés publics (notamment la transparence, la concurrence loyale et des critères objectifs) est sanctionné par la loi, il ne définit pas la notion de conflit d'intérêts.

Le DMP ne précise pas non plus la définition de conflit d'intérêts dans le cadre des marchés publics. Cependant, plusieurs articles traitent de la gestion des conflits d'intérêt. Le DMP précise, par exemple que les agents publics impliqués dans la passation, le contrôle ou l'exécution d'un marché public doivent se récuser lorsque leurs intérêts privés coïncident avec l'intérêt public et sont susceptibles d'influencer l'exercice normal de leurs fonctions. Le DMP mentionne par ailleurs qu'il est incompatible pour un agent de participer à la fois aux activités de passation d'un marché public et à son contrôle. Ainsi, par exemple, la qualité de membre et/ou de rapporteur d'une commission des marchés publics ou d'un jury de concours est incompatible avec celle de membre de la commission d'ouverture des plis et d'évaluation des offres. II est également mentionné que le service contractant ne peut pas attribuer un marché public à ses anciens employés pendant une période de quatre années. De plus, l'obligation de ne pas être en situation de conflit d'intérêts s'étend également aux soumissionnaires, qui doivent le signaler au service contractant le cas échéant. L'Algérie gagnerait donc à fournir une définition de la notion de conflit d'intérêts dans les marchés publics dans son cadre réglementaire. Des explications supplémentaires et des exemples concrets, y compris dans le domaine des marchés publics pourraient être développés au moyen de lignes directrices ou de notes d'informations afin d'assurer une meilleure compréhension du conflit d'intérêt. 
Par ailleurs, le cadre réglementaire ne précise pas clairement comment déclarer les conflits d'intérêt dans le cadre des marchés publics. L'article 88 du DMP prévoyant l'élaboration de code d'éthique et de déontologie des agents publics impliqués dans les marchés publics, précise l'obligation de signer une déclaration d'absence de conflit d'intérêt pour ces agents, dont le modèle doit être joint au code, lequel n'est toujours pas disponible. Le DMP ne précise ni la fréquence de signature de cette déclaration, ni à qui cette déclaration doit être transmise et qui doit l'analyser et la traiter. L'Algérie devrait donc préciser dans son cadre réglementaire comment déclarer les conflits d'intérêt. En plus du développement du code d'éthique et de déontologie des agents publics impliqués dans les marchés publics, l'Algérie devrait clarifier les dispositions relatives à la signature de déclaration d'absence de conflit d'intérêt afin de faciliter sa mise en place.

\section{Améliorer le système de déclaration de patrimoine}

Un système efficace de déclaration d'intérêt et de situations financières, y compris de patrimoine, peut jouer un rôle important dans la promotion de l'intégrité. En effet, il permet d'appuyer le processus de mise en place d'une culture d'intégrité et de renforcer la réédition de compte. Selon la manière dont ils sont conçus, les formulaires de déclaration peuvent être utilisés pour détecter les enrichissements illicites ou pour déterminer dans quelle mesure la décision d'un agent public a été compromise par des intérêts privés, comme par exemple un emploi précédent ou en dehors de la fonction publique.

De plus, en rendant publiques les déclarations d'intérêt et de situations financières, le gouvernement démontre sa volonté de transparence et permet un contrôle social, ajoutant ainsi un dispositif de surveillance supplémentaire. Des preuves empiriques récentes ont montré les effets positifs que peut avoir un système de déclaration sur la capacité d'un pays à contrôler la corruption (Vargas et Schlutz, 2016[27]).

En Algérie, d'après la loi $n^{\circ}$ 06-01, les agents publics occupant des postes ou fonctions supérieurs de l'État (y compris les sous-directeurs et directeurs ministériels) ont pour obligation de remplir une déclaration de patrimoine en début et en fin de fonction ou de mandat, ainsi qu'en cas de modification substantielle de patrimoine. II n'existe cependant pas de définition claire de la " modification substantielle de patrimoine », ce qui engendre des incertitudes et un manque d'efficacité du système.

Par ailleurs, en considérant les coûts des dispositifs de déclaration de patrimoine, une approche par les risques doit être adoptée afin d'arrêter la liste des fonctionnaires et agents publics soumis à l'obligation de renseigner la déclaration de patrimoine. Tous les agents impliqués dans le système de passation des marchés publics peuvent être soumis à des risques d'atteinte à l'intégrité, mais le cadre réglementaire ne prévoit l'obligation de déclaration que pour les «fonctionnaires supérieurs de l'État ». En effet, seuls quelques fonctionnaires impliqués dans les activités de marchés publics ont ce statut. Lors de la mission exploratoire, I'ONPLC a mentionné mener une réflexion pour rendre obligatoire la déclaration de patrimoine pour toutes les professions dites à risques y compris tous les agents impliqués dans le cycle de passation des marchés publics. En effet, l'Algérie devrait prendre des mesures afin d'élargir l'obligation de déclaration de patrimoine à tous les agents impliqués dans les marchés publics.

Conformément à ses attributions énoncées par la loi $n^{\circ} 06-01$, l'ONPLC est chargé de recueillir périodiquement les déclarations de patrimoine des agents publics, d'examiner et d'exploiter les informations qu'elles contiennent, et de veiller à leur conservation. L'ONPLC publie des statistiques basiques relatives à ces déclarations de patrimoine sur son site web, mais les dernières statistiques publiées datent de septembre 2015. De plus, selon le dernier bilan disponible en ligne, relatif à l'année 2015 , seuls $23 \%$ des 8000 fonctionnaires supérieurs assujettis à la déclaration ont rempli leur déclaration de patrimoine (ONPLC, s.d.[28]). Lors d'une journée d'étude sur le thème "Unis contre la corruption », le président de l'ONPLC a annoncé que l'Organe n'avait reçu en 2018 que 6000 déclarations émanant de hauts fonctionnaires de l'État, et fait état du manque de collaboration de certaines autorités publiques (Algérie Presse Service, s.d.[29]). Ceci peut s'expliquer par le fait que les personnes concernées doivent remplir manuellement la déclaration et la soumettre, selon les cas, à l'autorité de tutelle (pour les 
agents publics occupant des postes ou fonctions supérieurs de l'État), ou à l'autorité hiérarchique directe (pour les agents publics dont la liste est fixée par arrêté de l'autorité chargée de la fonction publique). Au vu du nombre de déclarations actuelles et futures, le traitement manuel de ces déclarations pose quelques problèmes d'efficacité et de confidentialité. Depuis 2015, I'ONPLC réfléchit à mettre en place une plateforme électronique de traitement des données en ligne (ONPLC, s.d.[28]) (Algérie Presse Service, s.d.[29]). L'Algérie gagnerait en effet à mettre en place dès que possible la plateforme électronique pour la déclaration de patrimoine afin de faciliter leur transmission par les agents publics, mais également pour mener des analyses détaillées et éventuellement identifier des signaux d'alerte.

\subsubsection{La gestion efficace et la transparence du système d'exclusion des fournisseurs (Blacklisting)}

L'exclusion des fournisseurs ayant des pratiques nuisant à l'intégrité du système montre une détermination forte et des objectifs concrets des gouvernements à lutter contre la corruption et préserver l'intégrité dans les marchés publics. Ce système fonctionne en tant que sanction envers les fournisseurs corrompus ou responsables d'actes d'atteinte à l'intégrité. C'est une pratique adoptée non seulement par les gouvernements, mais également par les banques et les institutions multilatérales de développement (de Koker et Harwood, 2015[30] $)$. L'encadré 2.6 décrit le système en place au Canada.

\section{Encadré 2.6. Le régime d'intégrité du gouvernement du Canada}

En 2015, le gouvernement du Canada a mis en place un régime afin de veiller à ce que les fournisseurs de l'État soient intègres et aient un comportement conforme aux normes d'éthique au Canada et à l'étranger.

Le ministère des Services publics et de l'Approvisionnement du Canada administre le régime d'intégrité pour le compte du gouvernement. II détermine si un fournisseur est admissible ou non à devenir fournisseur du gouvernement. Certaines infractions entraînent une inadmissibilité automatique, alors que d'autres nécessitent un examen au cas par cas. Généralement, ce système s'applique aux achats et aux transactions immobilières de plus de 10000 CAD (dollars canadiens).

Le régime contribue à encourager la mise en place de pratiques commerciales éthiques, assure une application régulière de la loi et maintient la confiance du public. II est transparent et rigoureux et correspond aux pratiques exemplaires en vigueur au Canada et à l'étranger. Le ministère a consulté et continue de consulter des associations de l'industrie et des spécialistes indépendants du domaine de l'éthique des achats.

Source : (Gouvernement du Canada, s.d. $[31])$.

En Algérie, conformément aux bonnes pratiques internationales, le cadre réglementaire régissant les marchés publics prévoit un système d'exclusion des fournisseurs. En effet, les articles 75 et 89 du DMP énoncent les situations pour lesquelles les opérateurs économiques peuvent être inscrits sur la liste des opérateurs économiques interdits de participer temporairement ou définitivement aux marchés publics (voir encadré 2.7). 


\section{Encadré 2.7. Les cas d'atteinte à l'intégrité pour lesquels les fournisseurs sont exclus de la participation aux marchés publics en Algérie}

En cas d'atteinte à l'intégrité du système de passation des marchés publics, les opérateurs économiques peuvent être exclus temporairement ou définitivement de la participation aux marchés publics. Cela concerne les fournisseurs qui :

- s'adonnent à des actes ou à des manœuvres tendant à promettre d'offrir ou d'accorder à un agent public, directement ou indirectement, pour lui-même ou pour une autre entité, une rémunération ou un avantage de quelque nature que ce soit, à l'occasion de la préparation, de la passation, du contrôle, de la négociation ou de l'exécution d'un marché public ou d'un avenant ;

- ont fait l'objet d'un jugement ayant autorité de la chose jugée et constatant un délit affectant leur probité professionnelle ;

- ne sont pas en règle avec leurs obligations fiscales et parafiscales ;

- ne justifient pas du dépôt légal de leurs comptes sociaux ;

- ont fait une fausse déclaration ;

- ont été inscrits sur la liste des opérateurs économiques interdits de participer aux marchés publics, prévue à l'article 89 du DMP ;

- ont été inscrits au fichier national des fraudeurs, auteurs d'infractions graves aux législations et réglementations fiscales, douanières et commerciales ;

- ont fait l'objet d'une condamnation pour infraction grave à la législation du travail et de la sécurité sociale.

Source : DMP.

Dans certains cas, en particulier en cas de corruption, en plus de l'inscription temporaire ou définitive du fournisseur sur la liste des opérateurs économiques interdits de participer aux marchés publics, le marché en cause ou l'avenant est résilié ou annulé. D'après le DMP et l'arrêté du 19 décembre 2015 fixant les modalités d'inscription et de retrait de la liste des opérateurs économiques interdits de participer aux marchés publics, la liste est tenue par l'ARMP, qui n'a toujours pas été mise en place. La liste est par ailleurs notifiée à l'ensemble des services contractants ou affichée sur le portail électronique des marchés publics, lequel n'a également toujours pas été mis en place. Par conséquent, cette liste n'est pas disponible en ligne.

D'après les missions exploratoires de l'OCDE, le ministère des Finances n'a recensé que quelques cas d'exclusion depuis un an, essentiellement dans les wilayas, et principalement pour des motifs de défaillances répétées et non d'atteinte à l'intégrité. Étant donné les volumes financiers en jeu et le haut risque d'atteinte à l'intégrité dans les marchés publics, l'inexistence d'opérateurs exclus pour cas d'atteinte à l'intégrité démontre les lacunes du système et son efficacité limitée pour atteindre les objectifs initiaux. C'est pourquoi, en attendant la mise en place de l'ARMP, le ministère des Finances devrait assurer la mise à jour effective de la liste d'exclusion des opérateurs économiques en coordination avec les services contractants et les autres corps de contrôle. Ceci pourrait se faire au travers d'une circulaire du ministère des Finances à destination de tous les services contractants soumis au DMP. II devrait par ailleurs s'assurer de la disponibilité en ligne de cette liste, qui devrait inclure les informations sur l'identité des opérateurs économiques et les motifs de leur exclusion, comme c'est le cas par exemple aux États-Unis 
avec le système de listes d'exclusion (Excluded Parties Lists System). Cette liste pourrait être disponible sur le site web du ministère des Finances.

\subsubsection{Renforcer les capacités dans le domaine de l'intégrité dans les marchés publics}

Les actions de renforcement des capacités en matière d'intégrité fournissent des conseils pratiques sur les comportements éthiques dans des situations où les règles officielles n'apportent pas de réponses claires sur la manière de se comporter dans des situations sensibles ou de dilemme éthique. Ces actions sont particulièrement nécessaires dans les contextes caractérisés par des niveaux de corruption élevés, où de nombreuses situations ne sont pas, ou ne peuvent pas être, définies par des règles officielles. Ainsi, les agents impliqués dans les marchés publics doivent bénéficier d'actions de renforcement des capacités spécialisées afin non seulement d'atténuer les risques de corruption, mais aussi de promouvoir des valeurs de service public (OCDE, 2019 $\left.9_{[14]}\right)$.

En Algérie, l'ARMP est chargée du renforcement des capacités dans les marchés publics (voir chapitre 6), mission qui comprend également le domaine de l'intégrité, mais elle n'a toujours pas été mise en place. Les actions de renforcement de capacités sont donc principalement menées par l'Institut supérieur de gestion et de planification (ISGP) dans le cadre de formations dans le domaine des marchés publics. L'offre de formation actuelle, en particulier celle de I'ISGP, ne couvre que de façon très limitée les aspects liés à l'intégrité dans les marchés publics. En effet, seul un certificat de maîtrise en réglementation des marchés publics inclut un module dédié à la déontologie et la prévention du risque pénal dans les marchés publics. Cela ne représente qu'un volet de l'intégrité dans les marchés publics. En effet, il ne s'agit pas simplement d'éviter les risques pénaux, mais surtout de promouvoir une bonne gouvernance et l'adhésion aux valeurs de service public.

Par ailleurs, l'ONPLC a également des attributions en termes de sensibilisation sur les risques de corruption, y compris dans le domaine des marchés publics. D'après les discussions menées avec I'ONPLC lors des missions exploratoires de l'OCDE, dans le cadre de son plan d'action 2012-14, l'organe a organisé quelques activités de sensibilisation et formation sur la lutte contre la corruption dans les marchés publics, cependant aucune donnée chiffrée n'a été transmise. De plus, l'ONPLC a affirmé avoir lancé en avril 2018, des sessions de formation générale sur la lutte contre la corruption au profit des 800 agents publics issus de 22 départements ministériels et exécutifs des wilayas. Par ailleurs, des initiatives au niveau local ont également vu le jour, par exemple, dans le cadre de la mise en œuvre du Programme national de sensibilisation et de formation anti-corruption dans le grand sud, l'ONPLC, en partenariat avec les autorités locales de la wilaya de Tamanghasset, a organisé du 25 au 27 décembre 2018 une session de formation sur les risques de corruption dans les marchés publics (ONPLC, 2019[32]). La mise en place de ces actions est positive, cependant des actions plus ciblées par domaine de risque et adoptant une approche plus positive de promotion des normes et valeurs d'intégrité pourrait avoir un impact plus positif. L'Algérie gagnerait donc à intégrer structurellement, dans toutes les actions de renforcement des capacités dans le domaine des marchés publics, un module consacré aux normes et valeurs d'intégrité, ainsi qu'aux mécanismes de lutte contre la corruption.

Par ailleurs, I'ISGP développe actuellement un module d'enseignement à distance (e-learning) avec I'ONPLC sur le thème de la prévention et de la lutte contre la corruption, qui sera disponible en français et en arabe. Cette initiative permettra de former un maximum d'agents publics et couvrir l'ensemble du territoire. L'ONPLC et l'ISGP devraient s'assurer de la disponibilité de ce module dans les plus brefs délais et de la mise à jour de ce module compte tenu des changements réglementaires prévus prochainement dans le domaine de la lutte contre la corruption et de l'intégrité, mais également de l'émergence de nouveaux risques dans ce domaine. 


\subsubsection{Favoriser la participation transparente et effective de toutes les parties prenantes du système de marchés publics, y compris le secteur privé et la société civile}

Une bonne gouvernance des marchés publics nécessite une collaboration entre toutes les parties prenantes impliquées dans le système, y compris le secteur privé et la société civile, qui peuvent jouer un rôle de surveillance et de contrôle des marchés publics. En agissant comme une sorte de contrôle social direct sur les activités du gouvernement, la société civile et les citoyens peuvent renforcer la transparence des activités du gouvernement et rétablir la confiance du public dans le système (OCDE, 2018[20]). La nécessité d'engager le secteur privé et la société civile pour développer une culture d'intégrité est également soulignée dans la Recommandation du Conseil de l'OCDE sur les marchés publics et dans la Recommandation du Conseil de l'OCDE sur l'intégrité publique.

En effet, il existe un lien étroit entre la corruption et le degré de faculté dont jouissent les organisations de la société civile à travailler sur et influencer les politiques publiques (Transparency International ${ }_{[7]}$ ). La participation de toutes les parties prenantes dans le système de marchés publics peut contribuer à favoriser non seulement la responsabilité des agents publics en agissant comme un corps de contrôle, mais également à renforcer l'intégrité dans toute la société en établissant et appliquant les valeurs et les normes du secteur public, y compris dans le système des marchés publics. De nombreux pays ont mis en place des mécanismes permettant de promouvoir la participation de la société civile dans les marchés publics (voir l'exemple du Mexique dans l'encadré 2.8).

\section{Encadré 2.8. Participation des « témoins sociaux » dans le processus de passation des marchés publics au Mexique}

Un « témoin social » est un représentant de la société civile mexicaine agissant comme observateur externe, dont la participation est obligatoire pour les processus d'appels d'offres ouverts au-dessus de certains seuils. En 2015, les seuils étaient 350 millions MXN (pesos mexicains) pour les marchés de biens et de services et de 710 millions MXN pour les travaux publics.

Le témoin social doit avoir bonne réputation, être connu et jugé digne de confiance, mais aussi indépendant des parties prenantes impliquées dans le processus de passation du marché public en question. Les témoins sociaux sont sélectionnés par une procédure d'appel d'offres ouvert pour une durée de trois ans.

En plus de permettre une surveillance publique du processus de passation des marchés publics, le témoin social peut faire des recommandations à titre consultatif, pendant et après le processus. II ou elle bénéficie d'un accès libre à l'information et aux documents relatifs au marché public concerné, et peut en particulier :

- vérifier le cahier des charges,

- participer en tant qu'observateur à toutes les réunions organisées avec les soumissionnaires, de manière à lever tout doute concernant leur déroulement,

- recevoir les déclarations d'intégrité unilatérales des différentes parties,

- observer la soumission des offres techniques et financières,

- participer en tant qu'observateur aux réunions pendant lesquelles le résultat d'une adjudication est annoncé.

II prépare un rapport avec des observations et des recommandations sur le processus qui est rendu public par le système de passation électronique des marchés publics au Mexique, CompraNet.

Sources : (OCDE, 2017[33]) ; (OCDE, 2016[34]). 
La participation de la société civile est également soulignée dans le cadre réglementaire de l'Algérie. L'article 15 de la loi relative à la prévention et à la lutte contre la corruption énonce que la participation de la société civile est encouragée notamment à travers : la transparence des processus de décision ; les programmes de sensibilisation et d'éducation; et l'accès effectif à l'information concernant la corruption par des médias et du public.

Cependant, en Algérie, le système actuel connaît deux lacunes principales. D'une part, il ne comporte aucune disposition visant à intégrer la société civile dans les processus de passation des marchés publics. D'autre part, lors des missions exploratoires de l'OCDE, certaines associations de la société civile travaillant dans le domaine de la lutte contre la corruption ont mentionné des difficultés à obtenir une accréditation du gouvernement. En effet, la Constitution de l'Algérie reconnaît le droit de réunion, mais de nombreuses études soulignent la limitation et les obstacles posés au développement de la société civile de par les multiples exigences de la loi et le manque de cohérence de son application (Ambassade des États-Unis en Algérie, 2016[35]).L'Algérie gagnerait donc à s'assurer de la participation des acteurs non publics dans le système tout au long du cycle de passation des marchés publics afin d'assurer son intégrité et sa transparence.

\subsubsection{Un système efficace de signalement de cas d'atteinte à l'intégrité}

Afin d'assurer l'intégrité dans le secteur public, y compris dans le domaine des marchés publics, l'OCDE recommande de mettre en place des dispositifs clairs pour le signalement aux autorités compétentes des soupçons crédibles de violation de ces lois et réglementations, sans crainte de représailles (OCDE, $\left.2015_{[3]}\right)$. Ces dispositifs doivent pouvoir être utilisés par toutes les parties prenantes, y compris la société civile et les citoyens.

Par ailleurs, il est de la responsabilité des pouvoirs publics d'établir des procédures claires pour signaler les comportements fautifs, par exemple un bureau des réclamations interne, une ligne d'appel directe, un médiateur extérieur ou un système de déclaration électronique protégeant l'anonymat de la personne qui signale une anomalie tout en permettant les demandes d'éclaircissements. L'une des principales difficultés est d'assurer la protection des fonctionnaires qui signalent une anomalie contre les représailles, en particulier au moyen d'une protection juridique, de la protection des informations relatives à leur vie privée, de l'anonymat, ou de la création d'une commission de protection. II faut en même temps veiller tout particulièrement à ce que la gestion des réclamations soit bien documentée et impartiale pour éviter de nuire indûment à la réputation des personnes touchées par les allégations (OCDE, 2011[36]).

Dans les marchés publics, plusieurs personnes ou parties prenantes peuvent être amenées à signaler des cas d'atteinte à l'intégrité : les corps de contrôle, les institutions publiques, les citoyens, les agents publics, mais également les opérateurs économiques. Le cadre réglementaire algérien régissant la lutte contre la corruption ne prévoit aucune disposition claire quant aux mécanismes de signalement des cas d'atteinte à l'intégrité. En effet, l'OCRC peut être saisi par l'Inspection générale des finances, la CTRF, les Commissions des marchés ou encore les services de police, mais pas par les citoyens. Bien que cela ne soit pas prévu dans le cadre réglementaire, l'ONCPL a mentionné lors des missions exploratoires de l'OCDE la possibilité pour les citoyens ou toute autre partie prenante de lui signaler des cas d'atteinte à l'intégrité. Seuls les cas dûment documentés et justifiés sont transmis à la justice. Aucune donnée statistique n'a été partagée sur ce sujet.

Par ailleurs, le système actuel ne prévoit aucune disposition pour protéger les lanceurs d'alerte, ce qui n'est pas conforme à la CNUCC et n'encourage pas le signalement. Cependant, l'Algérie prépare actuellement un projet de loi intégrant des dispositions relatives à la protection des lanceurs d'alerte. Le gouvernement algérien devrait donc mettre en place un système de signalement de cas d'atteinte à l'intégrité garantissant l'anonymat tout en protégeant la personne qui fait le signalement. 


\subsection{Appliquer des dispositifs de surveillance et de contrôle en intégrant des stratégies de management des risques dans les marchés publics}

La supervision et le contrôle des activités de passation des marchés publics sont essentiels pour favoriser la responsabilisation et promouvoir l'intégrité du système de passation des marchés publics ; de plus ces processus permettent de disposer d'indicateurs de performance du système.

La supervision et le contrôle constituent l'un des fondements des instruments de l'OCDE visant à promouvoir les systèmes d'intégrité efficaces dans les marchés publics et dans le secteur public dans son ensemble (OCDE, 2016[2]). Ces éléments sont soulignés notamment dans la Recommandation du Conseil de l'OCDE sur l'intégrité publique et la Recommandation du Conseil de l'OCDE sur les marchés publics. Cette dernière en particulier recommande aux gouvernements y adhérant d'« appliquer des dispositifs de surveillance et de contrôle visant à favoriser la responsabilité tout au long du cycle de passation des marchés publics avec, notamment, des procédures adaptées de contestation et de sanction » (OCDE, 2015 [3]).

\subsubsection{Accroître l'efficacité du système de contrôle appliqué aux marchés publics}

Un système de surveillance robuste comprend des institutions ainsi que des fonctions de contrôle internes et externes relevant des trois pouvoirs du gouvernement : l'exécutif, le législatif et le judiciaire. Le partage clair et efficace de leurs rôles constitue donc un élément clé afin de favoriser la responsabilité et la reddition des comptes tout en s'assurant de l'efficacité tout au long du cycle de passation des marchés publics, comme le souligne la Recommandation du Conseil de l'OCDE sur les marchés publics, (OCDE, 2015[3]). Deux types de contrôles sont généralement identifiés : les contrôles internes et les contrôles externes. Cette distinction dépend de l'appartenance de l'organe ou de l'entité de contrôle au pouvoir exécutif (entité publique, ministères, tutelle, etc.) ou au pouvoir législatif ou judiciaire. Il est également possible de définir les contrôles externes comme ceux effectués par des organismes "en dehors " de l'entité, bien qu'appartenant au pouvoir exécutif (INTOSAI, 2004 $[37]$ ). En Algérie, c'est cette dernière définition qui est retenue dans le cadre de cette analyse.

Le DMP définit trois types de contrôles appliqués aux processus de passation des marchés publics : le contrôle interne, le contrôle externe a priori et le contrôle externe a posteriori, qui interviennent à différentes étapes du processus, comme l'illustre le graphique 2.5 .

\section{Graphique 2.5. Le système de contrôles appliqués aux marchés publics en Algérie}

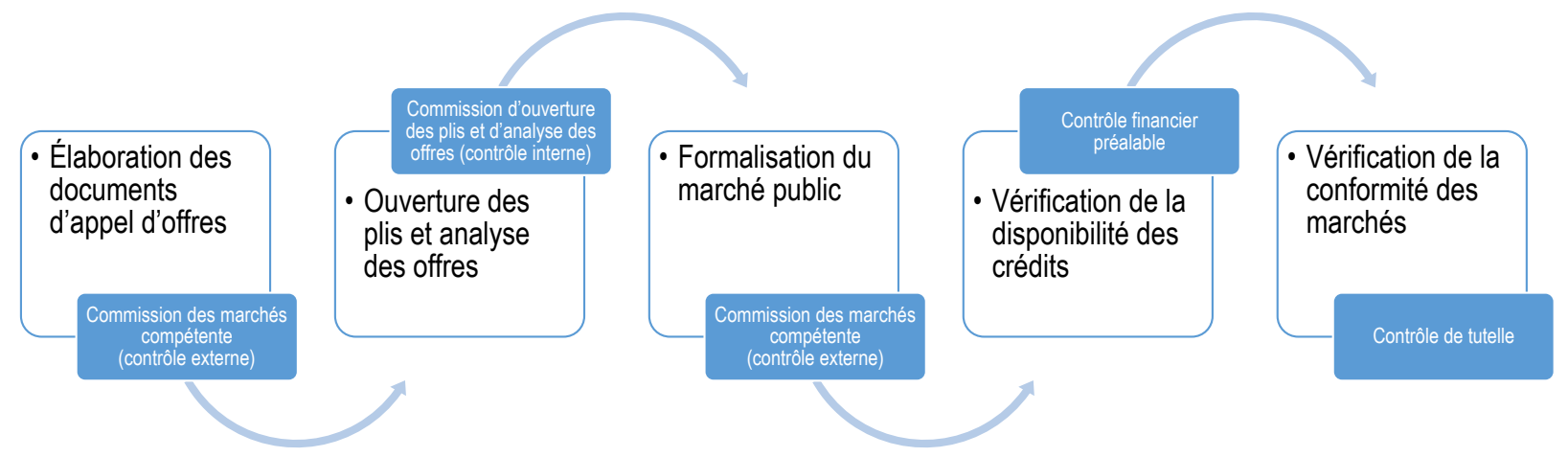




\section{Établir un système effectif des trois lignes de maîtrise afin d'améliorer le système de contrôle interne}

Le terme « système de contrôle interne » décrit l'ensemble des processus et mécanismes mis en œuvre par le management, la hiérarchie et le personnel d'une entité destinés à fournir une assurance raisonnable quant à la réalisation d'objectifs liés aux opérations, au reporting et à la conformité (COSO, 2013[38]).

Le système de contrôle interne distingue trois lignes de maîtrise au niveau de chaque entité publique, qui représentent des acteurs ou des fonctions chargés du système de contrôle interne (voir graphique 2.6). La première ligne de maîtrise concerne ceux qui mettent en œuvre les processus opérationnels et gèrent au quotidien les risques associés. La seconde ligne de maîtrise, le management, instaure des fonctions pour s'assurer que la première ligne de maîtrise est bien conçue et effective. Chacune de ces fonctions est, dans une certaine mesure, indépendante de la première ligne de maîtrise, mais il s'agit, par nature, de fonctions support du management. La troisième ligne est constituée par ceux qui donnent une assurance raisonnable et indépendante sur l'efficacité des processus et des mécanismes de contrôle interne, il s'agit de la fonction de l'audit interne (IIA, 2013[39]).

\section{Graphique 2.6.Le modèle des trois lignes de maîtrises du contrôle interne}

\section{Ministre / Conseil d'administration / Comité d'audit}

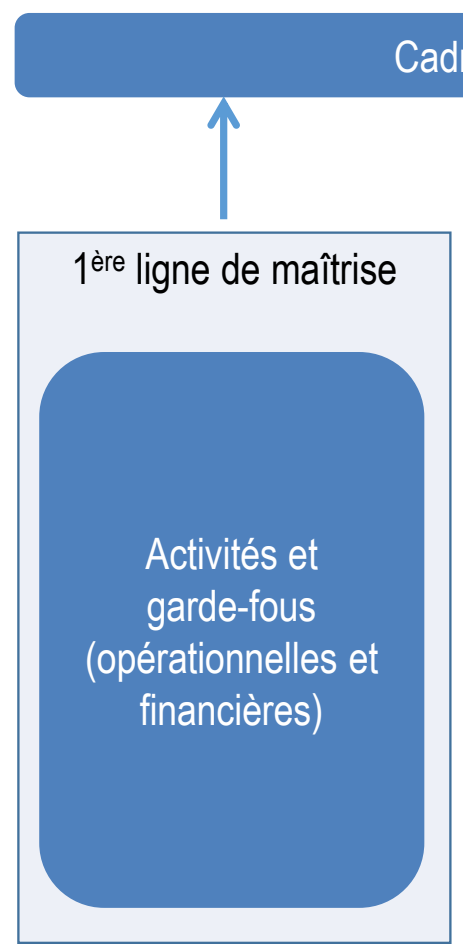

Cadres supérieurs
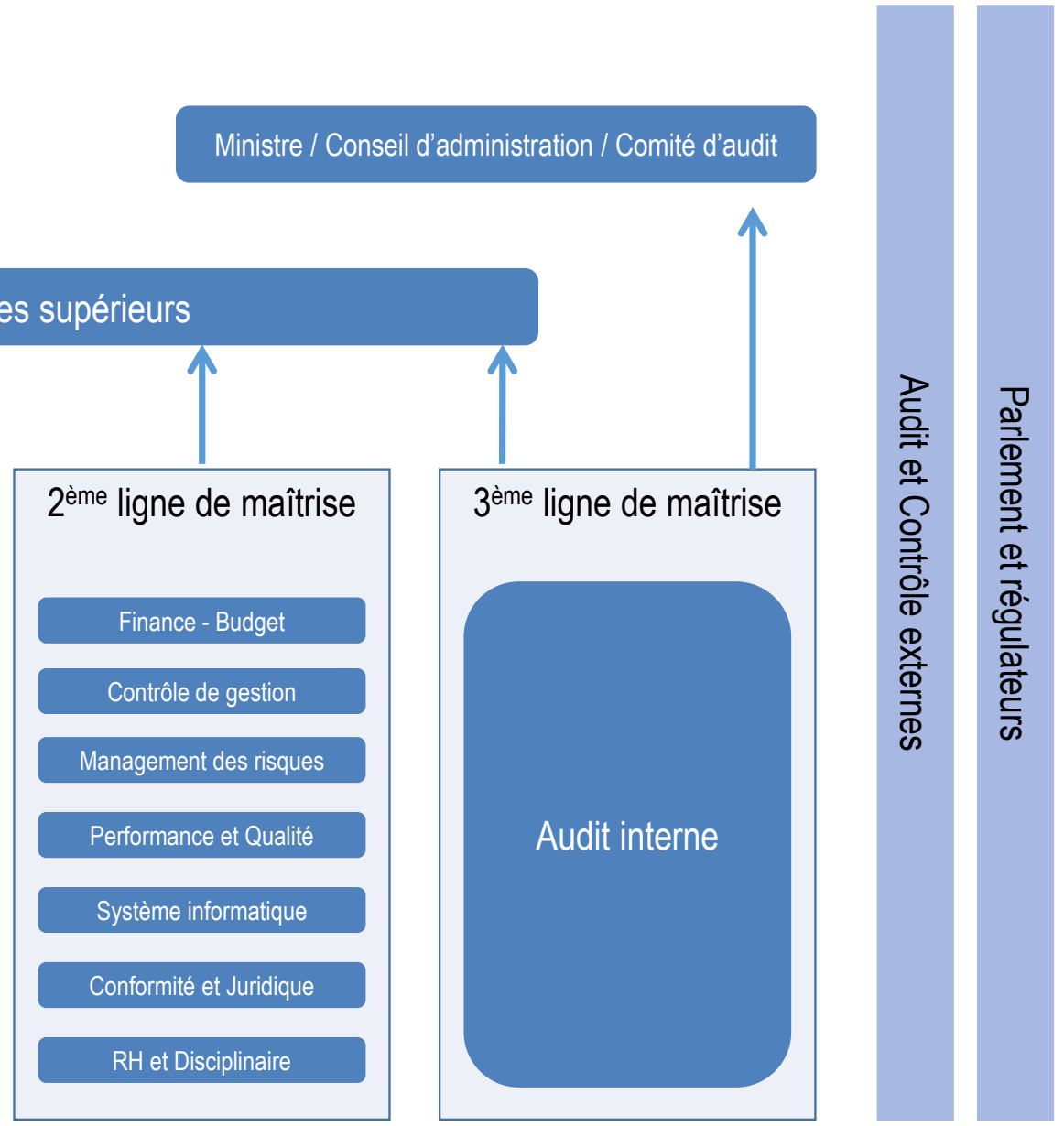

Source : adapté de (OCDE, 2016 $\left.{ }_{[40]}\right)$.

Le DMP ne mentionne que la Commission d'ouverture des plis et d'évaluation des offres comme mécanisme de contrôle interne spécifique aux marchés publics. Constituée par le service contractant, la 
commission effectue un travail administratif et technique qu'elle soumet au service contractant chargé d'attribuer le marché, de déclarer l'infructuosité de la procédure ou son annulation ou l'annulation de l'attribution provisoire du marché. Elle émet à ce titre, un avis motivé. Les services contractants peuvent instituer sous sa responsabilité un comité technique chargé de l'élaboration du rapport d'analyse des offres pour les besoins de la commission d'ouverture des plis et d'évaluation des offres, qui constituera un mécanisme de contrôle interne supplémentaire. Bien que ces mesures soient bien des mécanismes de contrôle internes, ils sont insuffisants et non exhaustifs.

Si l'on transpose le modèle des trois lignes de maîtrise dans le système de passation des marchés publics en Algérie, pour chaque entité publique, la première ligne de maîtrise est composée par les agents en charge de la rédaction des spécifications techniques de la rédaction des documents d'appel d'offres et du suivi d'exécution du marché. La commission d'ouverture des plis et d'évaluation des offres, ainsi que le comité technique, font partie de la seconde ligne de maîtrise (ainsi que de nombreuses autres fonctions, financières, budgétaires, etc.).La responsabilité de la troisième ligne de maîtrise dans un système de contrôle interne - l'audit interne - qui est d'examiner, d'évaluer et de suivre l'adéquation et l'efficacité des processus et des mécanismes de contrôle interne (COSO, 2013 ${ }_{[38]}$ ), n'est pas clairement attribuée dans le système algérien de passation des marchés publics. L'Algérie devrait donc établir un système effectif des trois lignes de maitrise afin d'améliorer son système de contrôle interne et donc l'efficacité des marchés publics. Pour cela le pays devra établir une fonction d'audit interne de façon à assurer la mise en place des processus de contrôle interne et leur efficacité pour atténuer les risques, y compris dans le domaine des marchés publics. Par ailleurs, l'Algérie devra également développer des lignes directrices ou une circulaire afin de clarifier différentes fonctions et mécanismes du contrôle interne dans le contexte algérien, et attribuer des rôles et des responsabilités claires à travers les trois lignes de maîtrise.

Par ailleurs, la Commission d'ouverture des plis et d'évaluation des offres procède à l'ouverture des plis en séance publique, en présence des soumissionnaires. Un des objectifs de cette commission et du comité technique est d'analyser et d'évaluer les offres d'une manière objective, et donc d'atténuer les risques d'atteintes à l'intégrité. Cependant, une telle interaction physique pourrait accentuer ces risques et, de surcroît, augmenter le risque de collusion (OCDE, 2012[41]). L'Algérie gagnerait à rendre cette étape électronique une fois le système électronique de passation des marchés publics est mis en place.

\section{Établir les canaux de coordination entre les différentes instances de contrôle externe et développer un mécanisme de suivi de leurs observations}

Les différents mécanismes de contrôle externe ont pour but d'assurer que les systèmes de gouvernance et les processus opérationnels des organisations publiques sont fondés sur les impératifs d'économie, d'efficience et d'efficacité, et que les dispositifs de contrôle interne sont bien conçus et fonctionnent convenablement pour atténuer les risques menaçant la réalisation des objectifs de l'organisation (INTOSAI, 2004[37]).

En Algérie, plusieurs entités sont en charge du contrôle externe a priori et a posteriori. D'après le DMP, les contrôles externes a priori sont réalisés par une commission des marchés. Instituée auprès de chaque service contractant, cette commission est compétente pour apporter son assistance en matière de préparation et de formalisation des marchés publics, examiner les projets de cahiers des charges, de marchés et d'avenants, et examiner les recours introduits par les soumissionnaires, dans les conditions fixées à l'article 82 du DMP. Différentes commissions de marchés interviennent selon les seuils et les types de marchés (tableau 2.1). 
Tableau 2.1. Les seuils de compétence des commissions des marchés par types de marchés

\begin{tabular}{|c|c|c|c|c|}
\hline & Travaux & Fournitures & Services & Études \\
\hline $\begin{array}{l}\text { Commission sectorielle } \\
\text { des marchés }\end{array}$ & $\begin{array}{l}>1 \text { milliard DZD } \\
\text { et } \\
>12 \text { millions DZD (pour } \\
\text { l'administration centrale) }\end{array}$ & $\begin{array}{l}>300 \text { millions DZD } \\
\text { et } \\
>12 \text { millions DZD (pour } \\
\text { l'administration centrale) }\end{array}$ & $\begin{array}{l}>200 \text { millions DZD } \\
\text { et } \\
>6 \text { millions DZD (pour } \\
\text { l'administration centrale) }\end{array}$ & $\begin{array}{l}>100 \text { millions DZD } \\
\text { et } \\
>6 \text { millions DZD (pour } \\
\text { l'administration centrale) }\end{array}$ \\
\hline $\begin{array}{l}\text { Commission régionale } \\
\text { des marchés }\end{array}$ & $<1$ milliard DZD & $<300$ millions DZD & $<200$ millions DZD & $<100$ millions DZD \\
\hline $\begin{array}{l}\text { Commission des } \\
\text { marchés de Wilaya }\end{array}$ & $\begin{array}{l}=\text { ou }<1 \text { milliard DZD } \\
\text { et } \\
>\text { ou = } 200 \text { millions DZD } \\
\text { (pour les marchés des } \\
\text { communes) }\end{array}$ & $\begin{array}{l}=\text { ou }<300 \text { millions DZD } \\
\text { et } \\
>\text { ou }=200 \text { millions DZD } \\
\text { (pour les marchés des } \\
\text { communes) }\end{array}$ & $\begin{array}{l}=\text { ou }<300 \text { millions DZD } \\
\text { et } \\
>\text { ou = } 50 \text { millions DZD } \\
\text { (pour les marchés des } \\
\text { communes) }\end{array}$ & $\begin{array}{l}=\text { ou }<100 \text { millions DZD } \\
\text { et } \\
>\text { ou }=20 \text { millions DZD } \\
\text { (pour les marchés des } \\
\text { communes) }\end{array}$ \\
\hline $\begin{array}{l}\text { Commission des } \\
\text { marchés du service } \\
\text { contractant }\end{array}$ & $=$ ou $<1$ milliard DZD & $=$ ou $<300$ millions DZD & $=$ ou $<300$ millions DZD & $=$ ou $<100$ millions DZD \\
\hline $\begin{array}{l}\text { Commission } \\
\text { communale des } \\
\text { marchés }\end{array}$ & $<200$ millions DZD & $<200$ millions DZD & $<50$ millions DZD & $<20$ millions DZD \\
\hline $\begin{array}{l}\text { Commission des } \\
\text { marchés de } \\
\text { l'établissement public } \\
\text { local }\end{array}$ & $<50$ millions DZD & $<50$ millions DZD & $<50$ millions DZD & $<20$ millions DZD \\
\hline
\end{tabular}

Source : DMP.

Selon la composition de la commission des marchés définie par le DMP, un représentant du service contractant est membre de la commission, ce qui remet en question le caractère " externe »de cette commission, ainsi que l'indépendance du contrôle qu'elle réalise. En effet, d'après les Principes de l'OCDE pour renforcer l'intégrité dans les marchés publics, les chaînes de responsabilité sont nécessaires afin de définir le pouvoir d'approbation d'un marché public en assurant une séparation des tâches appropriée (OCDE, 2011[36]). Ce type de mécanisme de contrôle peut être considéré comme externe uniquement si le représentant du service contractant n'a aucun lien avec les procédures soumises à la commission. Afin de caractériser le contrôle opéré par la commission des marchés comme "externe ", l'Algérie doit s'assurer de l'indépendance des membres de la commission des marchés représentants le service contractant ; dans le cas contraire cette commission devra être considérée comme un mécanisme de contrôle interne.

D'autres types de contrôles externes sont exercés a posteriori, principalement par la Cour des comptes et I'Inspection générale des finances (IGF). L'IGF exerce un contrôle administratif des finances publiques. Son rôle et ses capacités d'intervention ont été renforcés à la suite des décisions des pouvoirs publics en 2006 de renforcer le contrôle sur la gestion et l'utilisation des fonds publics en général. Les gestionnaires sont ainsi tenus de répondre aux contestations et observations contenues dans les rapports de l'IGF. Ces rapports sont également communiqués aux autorités hiérarchiques ou de tutelles des entités publiques. L'IGF prépare également un rapport annuel portant sur les suites réservées à ses contestations et recommandations, y compris dans le domaine des marchés publics (Ministère des Finances, Algérie, 2011 [42]). Cependant, les rapports de l'IGF ne sont pas accessibles au public, ce qui n'est pas conforme à la bonne pratique internationale (en France, par exemple, l'IGF publie certains rapports sur son site afin d'assurer la transparence du système et la reddition des comptes (Inspection Générale des Finances, France, s.d.[43])). Par ailleurs, dans le cadre de cette revue, l'équipe OCDE n'a pas reçu l'extrait du rapport relatif aux marchés publics qu'elle avait sollicité afin d'analyser les dysfonctionnements identifiés par l'IGF. Pour améliorer la transparence du système, l'Algérie pourrait donc publier les rapports de l'IGF, tout en respectant les exigences de protection des données personnelles ou confidentielles. 
En outre, I'IGF dispose d'un logiciel d'audit des marchés publics, complété de plusieurs fiches d'audit concernant les différentes étapes et aspects d'un processus de passation des marchés publics. Bien qu'un tel outil puisse améliorer l'efficacité du système de contrôle en standardisant les procédures, ce logiciel basé sur la réglementation des marchés publics datant de 2008 n'est pas conforme à la réglementation en vigueur. L'IGF devrait donc le mettre à jour.

La Cour des comptes exerce également un contrôle externe a posteriori afin de contribuer à la bonne gouvernance des finances publiques. D'après le cadre réglementaire, elle peut mener, entre autres, des contrôles de la discipline budgétaire, de la qualité et de la performance, et des évaluations des politiques publiques. Elle joue un rôle d'accompagnement pédagogique et répressif (la Cour peut appliquer des sanctions en agissant comme une juridiction). La Cour des comptes a développé des guides de contrôle basés sur une cartographie de risques dans la gestion des marchés publics qui lui permettent de mettre en priorité les processus et les institutions à contrôler ainsi que les aspects d'un processus. En se basant sur ces guides, la Cour prépare des rapports d'observations. Cependant ces guides et les rapports ne sont pas disponibles en ligne et n'ont pas été transmis à l'équipe de l'OCDE. Comme pour les rapports de l'IGF, la publication des rapports de la Cour des comptes pourrait renforcer la transparence et l'efficacité des mécanismes de contrôle en matière de passation des marchés publics, et ainsi contribuer à restaurer la confiance des citoyens.

Enfin, d'après le DMP un dernier contrôle externe est effectué par l'autorité de tutelle pour vérifier que les marchés passés par le service contractant sont conformes aux objectifs d'efficacité et d'économie, et s'assurer que l'objet du marché entre effectivement dans le cadre des programmes et priorités assignés au secteur (ce contrôle s'apparente donc à un audit de performance). Les tendances internationales montrent que les organes de contrôle conformément à l'évolution des critères qui définissent la performance publique ont intégré l'économie, l'efficience et l'efficacité ainsi que les impacts des actions publiques dans les objectifs de contrôle (Olivieri et al., 2015[44]). Cependant aucune information n'a pu être transmise à l'équipe OCDE pour lui permettre d'analyser l'efficacité de ce dispositif et sa contribution à l'amélioration de l'efficience du système. Par ailleurs, d'autres entités de contrôle externe, en particulier la Cour des comptes, devraient effectuer des audits de performance.

Les missions exploratoires de l'OCDE relèvent d'une part que les organes de contrôle a posteriori constatent souvent des irrégularités dans les marchés publics, alors même que ces activités peuvent être soumises à plus de cinq contrôles, et d'autre part que les entités publiques n'implémentent pas forcément les recommandations des organes de contrôle. Cependant, la non-disponibilité des rapports des organes de contrôle empêche des analyses plus détaillées sur les irrégularités et les erreurs et surtout sur l'efficacité du système de contrôle dans les marchés publics. Par ailleurs, il n'existe pas de règle ou de mécanisme établi de communication entre les différentes instances de contrôle qui partagent des objectifs communs, y compris dans le domaine des marchés publics. Or la Recommandation du Conseil de l'OCDE sur les marchés publics souligne l'importance de réduire les doublons et d'assurer une surveillance suffisante, ce pour quoi la coordination entre les organes de contrôle est essentielle. L'Algérie gagnerait donc à établir un système de communication entre les instances de contrôle, ainsi qu'un système robuste de suivi des plans d'action pour mettre en œuvre les recommandations des audits afin d'améliorer l'impact et la valeur ajoutée des mécanismes de contrôle.

\subsubsection{Développer une stratégie de management des risques}

Un risque est une condition ou une circonstance future incertaine qui pourrait avoir un impact sur l'atteinte des objectifs si elle se produit (ISO, 2018[45]). Les risques pouvant impacter le bon fonctionnement du système de passation des marchés publics existent pour chacun des processus de chacune des phases du cycle de passation des marchés publics. En effet, les marchés publics sont confrontés à différentes catégories de risques, notamment les risques financiers, organisationnels, sociétaux, du marché, ainsi que 
les risques d'atteinte à l'intégrité. Le graphique 2.7 présente certains risques d'atteinte à l'intégrité tout au long du cycle de passation des marchés publics.

\section{Graphique 2.7. Risques d'atteinte à l'intégrité dans le processus de passation des marchés}

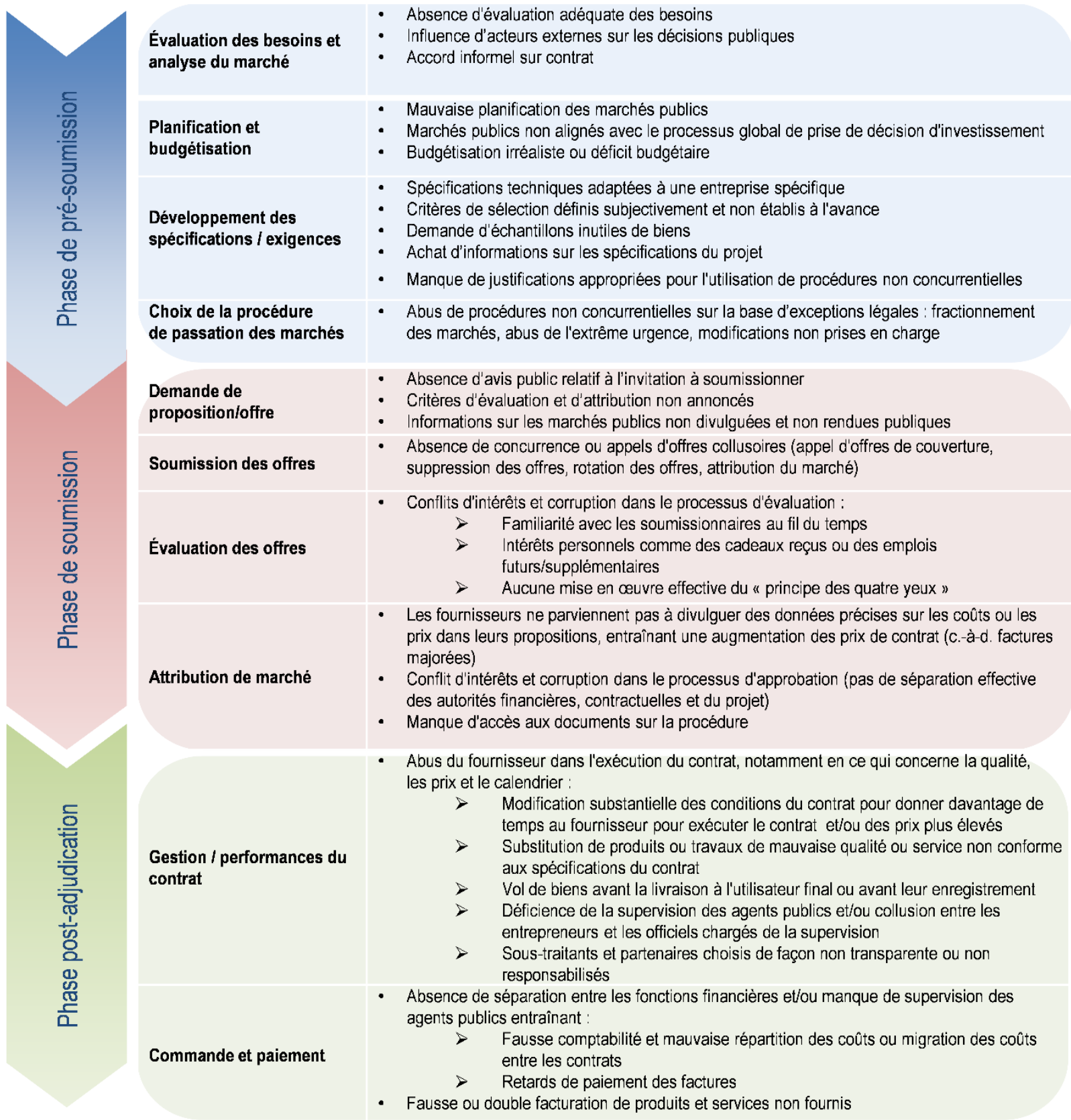

Source : (OCDE, 2016[2]).

L'appréciation des risques dans les processus gouvernementaux et leur environnement, y compris dans les marchés publics, doit être la base d'un système de supervision et de contrôle. Un système de supervision et de contrôle basé sur une analyse des risques solide apporte plus d'efficience et d'efficacité en permettant de concentrer les contrôles sur les activités à hauts risques et en mettant en place des moyens de maîtrise des risques qui permettent de réduire l'impact ou la probabilité de ces risques. Ainsi, 
le management des risques englobe non seulement l'identification et l'évaluation des risques (y compris une évaluation de la nature, des causes et des conséquences potentielles des risques) mais également l'atténuation des risques avec une communication et un suivi appropriés (graphique 2.8).

\section{Graphique 2.8.Cycle de management des risques selon les standards internationaux (ISO 31000:2018)}

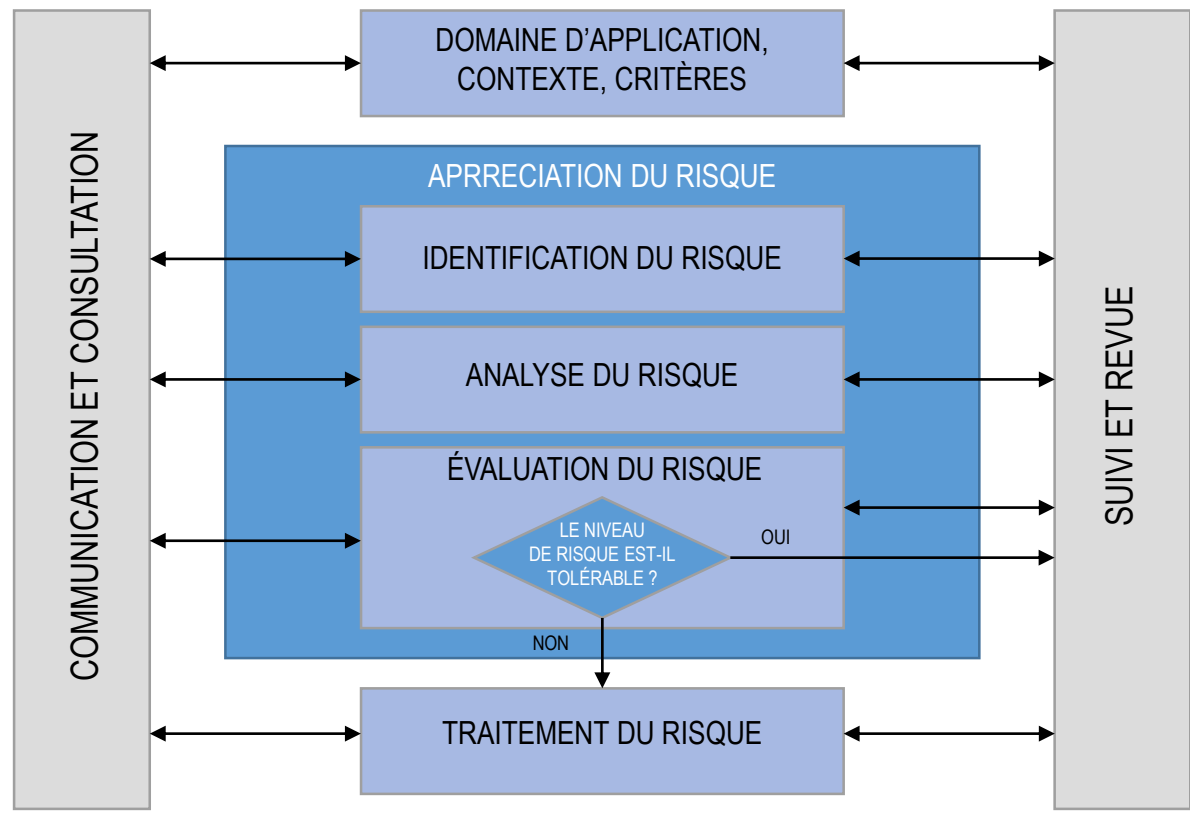

ENREGISTREMENT ET ÉLABORATION DES RAPPORTS

Source : (ISO, 2018[44]).

Un processus de passation efficace et efficient est crucial pour permettre à l'acheteur public de mener à bien sa mission de fourniture des services et infrastructures essentiels aux citoyens. À cette fin, un système de contrôle effectif, basé sur une fonction solide de management des risques est essentiel et contribue également à préserver l'intégrité du processus de passation des marchés publics. La mise en place d'un cadre ou d'une stratégie de management des risques est nécessaire afin d'implanter une culture de risque dans l'environnement spécifique des marchés publics et de fournir les outils nécessaires aux entités publiques pour atténuer ces risques. Plusieurs pays et entités publiques ont mis en place de telles stratégies, c'est le cas par exemple de la Tunisie qui, avec le concours de l'OCDE, a développé une stratégie de management des risques spécifiques aux marchés publics (HAICOP, 2019[46]).

En Algérie, le management des risques est pris en charge par les différents organes de contrôle, notamment les commissions des marchés, les contrôleurs financiers et les comptables publics. Lors des missions exploratoires de l'OCDE, la Cour des comptes a affirmé avoir développé une cartographie des risques pour mener ses contrôles, qui couvre également le domaine des marchés publics. De plus, I'ONPLC, en coordination avec le ministère des Finances, a engagé une étude pour élaborer une cartographie des risques de corruption afin: d'établir des indicateurs standards de risques de corruption ; de comparer et évaluer la manière dont les risques sont circonscrits et traités et aider à choisir les bonnes approches; de parfaire le mécanisme administratif permettant de contenir les risques de corruption; et d'identifier la nature des risques qui persistent malgré la mise en place de stratégies destinées à les contenir. Cependant, cette cartographie ne couvre pas les marchés publics. 
L'Algérie ne dispose pas de stratégie ou coordination centrale dans le domaine de management des risques dans les marchés publics pour les acheteurs publics. Afin d'améliorer l'efficience et l'efficacité du système de passation des marchés publics, l'Algérie devrait considérer le développement d'une stratégie de management des risques dans ce domaine en prenant en compte toutes les catégories de risques auxquelles les marchés publics peuvent être exposés.

\subsubsection{Assurer un système de sanctions proportionnelles et effectives en cas d'atteinte à l'intégrité dans les marchés publics}

Un système de sanction proportionnel et efficace suite à la détection d'un comportement illicite est un élément essentiel du système d'intégrité d'un pays. Les cadres réglementaires doivent donc prévoir un tel système pour les agents publics impliqués dans les activités de marchés publics, mais aussi pour le secteur privé.

Un système de sanction devrait agir comme un moyen de dissuasion pour les actes d'atteinte à l'intégrité ou de corruption, sans créer de crainte excessive ni une aversion au risque chez les parties prenantes (OCDE, 2018[47]). Les mécanismes de répression qui apportent des réponses appropriées pour tous les cas de violation présumée des normes d'intégrité incluent en général des sanctions à caractère disciplinaire, administratif, civil et/ou pénal (OCDE, 2017 $[4]$ ). Par ailleurs, pour le secteur privé, en plus des sanctions pénales, d'autres sanctions peuvent être appliquées telles que l'annulation du contrat public ou l'exclusion de la participation aux marchés publics (voir section 2.2). L'analyse des sanctions disciplinaires et pénales appliquées peut également être utilisée pour améliorer les mécanismes de contrôle et de surveillance, mais aussi de prévention, afin de renforcer la stratégie d'intégrité dans les marchés publics (OCDE, 2017[16] $)$. En effet, des statistiques élevées pour une catégorie précise de sanctions pourrait nécessiter des actions concrètes afin d'identifier les zones à haut risque et de mettre en place des actions afin de diminuer le nombre de cas.

En Algérie, des sanctions pénales sont prévues en cas d'actes portant atteinte à l'intégrité du système pour les agents publics, mais également pour les entreprises privées. En effet la loi relative à la prévention et à la lutte contre la corruption prévoit des sanctions pénales en cas d'atteinte à l'intégrité en relation directe ou indirecte avec les marchés publics allant de 6 mois à 20 ans d'emprisonnement et une amende allant de 50000 à 2000000 DZD (dinars algériens), la peine la plus sévère étant prévue pour les cas de corruption dans les marchés publics (voir tableau 2.2).

\section{Tableau 2.2. Principales sanctions pénales en relation avec les activités de marchés publics}

\begin{tabular}{|c|c|c|}
\hline & Peine d'emprisonnement & Amende \\
\hline Avantages injustifiés dans les marchés publics & De 2 à 10 ans & De 200000 à 1 million DZD \\
\hline Corruption dans les marchés publics & De 10 à 20 ans & De 1 million à 2 millions DZD \\
\hline Soustraction ou usage illicite de biens par un agent public & De 2 à 10 ans & De 200000 à 1 million DZD \\
\hline Concussion & De 2 à 10 ans & De 200000 à 1 million DZD \\
\hline Trafic d'influence & De 2 à 10 ans & De 200000 à 1 million DZD \\
\hline Abus de fonctions & De 2 à 10 ans & De 200000 à 1 million DZD \\
\hline Conflit d'intérêt & De 6 mois à 2 ans & De 50000 à 200000 DZD \\
\hline Prise illégale d'intérêts & De 2 à 10 ans & De 200000 à 1 million DZD \\
\hline $\begin{array}{l}\text { Cadeau ou tout avantage indu susceptible de pouvoir influencer le } \\
\text { traitement d'une procédure ou d'une transaction liée à ses fonctions }\end{array}$ & De 6 mois à 2 ans & De 50000 à 200000 DZD \\
\hline
\end{tabular}

Source : Loi 06-01 du 20 février 2006 relative à la prévention et à la lutte contre la corruption 
Les sanctions disciplinaires sont prévues par le statut général de la fonction publique (SGFP) et les statuts particuliers spécifiques à chaque secteur. En effet, les fautes professionnelles donnent lieu à des sanctions disciplinaires proportionnelles à la gravité de la faute commise et sont définies comme énoncées au tableau 2.3.Par ailleurs, des commissions de recours, composées pour moitié de représentants de l'administration et pour moitié de représentants élus de fonctionnaires, sont instituées dans chaque entité publique. Ces commissions peuvent être saisies par le fonctionnaire, pour les sanctions disciplinaires des $3^{\mathrm{e}}$ et $4^{\mathrm{e}}$ degré. Plusieurs de ces fautes professionnelles peuvent être liées aux activités de marchés publics.

Tableau 2.3. Les fautes professionnelles et les sanction disciplinaires applicables dans la fonction publique en Algérie

\begin{tabular}{|c|c|c|}
\hline & Fautes professionnelles & Sanctions disciplinaires \\
\hline 1er degré & $\begin{array}{l}\text { - Tout manquement à la discipline générale susceptible } \\
\text { de porter atteinte au bon fonctionnement du service }\end{array}$ & $\begin{array}{l}\text { - le rappel à l'ordre } \\
\text { - l'avertissement écrit } \\
\text { - le blâme }\end{array}$ \\
\hline $2^{\mathrm{e}}$ degré & $\begin{array}{l}\text { - Porte préjudice par imprudence ou négligence à la } \\
\text { sécurité des personnels et/ou des biens de } \\
\text { l'administration; } \\
\text { - Transgresse des obligations statutaires autres que } \\
\text { celles qui définissent des fautes professionnelles du } 3^{e} \text { et } \\
4 \text { e degré }\end{array}$ & $\begin{array}{l}\text { - la mise à pied de } 1 \text { à } 3 \text { jours } \\
\text { - la radiation du tableau d'avancement }\end{array}$ \\
\hline 3e degré & $\begin{array}{l}\text { - Se rend coupable de détournement de documents de } \\
\text { service } \\
\text { - Dissimule des informations d'ordre professionnel qu'il } \\
\text { est tenu de fournir dans l'exercice de ses fonctions } \\
\text { - Divulgue ou tente de divulguer des secrets } \\
\text { professionnels }\end{array}$ & $\begin{array}{l}\text { - la mise à pied de } 4 \text { à } 8 \text { jours } \\
\text { - l'abaissement d'un ou de deux échelons } \\
\text { - le déplacement d'office }\end{array}$ \\
\hline $4^{e}$ degré & $\begin{array}{l}\text { - Bénéficie d'avantages, de quelque nature que ce soit, } \\
\text { de la part d'une personne physique ou morale, en } \\
\text { contrepartie d'un service rendu dans le cadre de } \\
\text { l'exercice de ses fonctions }\end{array}$ & $\begin{array}{l}\text { - la rétrogradation dans le grade immédiatement inférieur } \\
\text { - le licenciement }\end{array}$ \\
\hline
\end{tabular}

Source : Statut général de la fonction publique.

Malgré l'existence dans le cadre réglementaire de sanctions pénales et disciplinaires, aucune statistique ou données ne sont disponibles quant à l'application effective de ces sanctions. L'Algérie devrait donc, d'une part, faire le nécessaire pour assurer l'application du système de sanction prévu dans le cadre réglementaire et, d'autre part, assurer l'analyse et la communication de ces données afin d'identifier les zones à haut risque dans le domaine des marchés publics et améliorer le système de contrôle.

\subsubsection{Mettre en place un système équitable et transparent de recours et de contestation de l'attribution d'un marché}

Un système de recours effectif permet aux opérateurs économiques de demander l'application des dispositions du cadre réglementaire régissant les marchés publics et de leurs droits dans le cas où une entité publique, volontairement ou involontairement, ne s'y est pas conformée (OCDE, 2011[48]). Toutes les parties prenantes dans le processus de marchés publics, y compris les fournisseurs, les soumissionnaires potentiels et la société civile, doivent avoir accès à des mécanismes efficaces d'examen et de recours si elles estiment que le processus d'acquisition a été effectué en violation du cadre réglementaire. De tels mécanismes assurent la bonne gouvernance des marchés publics et renforcent la confiance des entreprises dans le système de passation des marchés publics tout en veillant à ce que le processus des marchés publics soit équitable, transparent et conforme à la législation (OCDE, 2018[47]). 
Dans un premier temps, les fournisseurs potentiels devraient pouvoir soumettre leur plainte à l'entité publique afin de prévenir les conflits et d'anticiper le coût d'une action quasi-judiciaire ou judiciaire (OCDE, $\left.2011_{[36]}\right)$. Dans certains pays, un comité indépendant de règlement des plaintes permet de résoudre les plaintes avant le recours à la voie judicaire. Par exemple, la Norvège dispose ainsi du KOFA (Klagenemnda for offentlige anskaffelser), un comité constitué de dix membres. Le recours à ce comité est payant afin d'éviter le dépôt abusif de plaintes. Bien que ses décisions ne soient pas contraignantes, il jouit d'une certaine notoriété et d'un respect qui rendent ce système efficace.

Par la suite, les entités publiques doivent pouvoir se retourner vers une autorité de recours, qui peut être spécialisée ou non dans les marchés publics. Les systèmes de recours doivent être rapides, efficaces, transparents et non discriminatoires ; ils doivent être analysés par un organisme indépendant de l'entité adjudicatrice et n'ayant aucun intérêt dans la procédure en question (OCDE, 2013[49]). Cette approche est appuyée sur le plan international par différentes organisations internationales (voir encadré 2.9).

\section{Encadré 2.9. Standards internationaux en matière de recours dans le domaine des marchés publics}

\section{Convention des Nations Unies contre la corruption}

Les systèmes appropriés de marchés publics doivent prévoir : "Un système de recours interne efficace, y compris un système d'appel efficace, qui garantisse l'exercice des voies de droit en cas de non-respect des règles ou procédures établies conformément au présent paragraphe ».

\section{Organisation mondiale du commerce}

« Chaque Partie établira une procédure de recours administratif ou judiciaire s'appliquant en temps opportun, efficace, transparente et non discriminatoire au moyen de laquelle un fournisseur pourra déposer un recours $»$.

\section{Directive européenne de recours}

Les États membres s'assurent que les procédures de recours sont accessibles, selon des modalités que les États membres peuvent déterminer, au moins à toute personne ayant ou ayant eu un intérêt à obtenir un marché déterminé et ayant été ou risquant d'être lésée par une violation alléguée.

\section{Recommandation du Conseil de l'OCDE sur les marchés publics}

« Les Adhérents devraient :[...]iii) Traiter les contestations avec équité, rapidité et transparence grâce à l'établissement de voies de recours efficaces permettant de contester les décisions relevant du domaine des marchés publics en vue de corriger les défaillances, de prévenir le non-respect des règles et d'instaurer la confiance des soumissionnaires, y compris les concurrents étrangers, quant à l'intégrité et l'équité du système de passation de marchés publics. D'autres éléments essentiels d'un système de contestation efficace sont un dispositif d'examen spécifique et indépendant et des réparations adaptées ».

Sources : (OMC (Organisation Mondiale du Commerce), s.d.[50]) ; (UNODC, 2004[51]) ; (Conseil des Communautés Européennes, 1989[52]).

En Algérie, les soumissionnaires peuvent déposer un recours auprès de la commission des marchés compétente dans les dix jours à compter de la première publication de l'avis d'attribution provisoire du marché, dans les cas suivants : attribution provisoire de marché, déclaration d'infructuosité d'un appel d'offres, annulation d'une attribution provisoire ou de l'appel d'offres (voir tableau 2.1).Par ailleurs, d'après le Code de procédure civile, le tribunal administratif peut être saisi par requête avant la conclusion d'un 
contrat en cas de manquement aux obligations de publicité et de mise en concurrence auxquelles est soumise la passation des contrats administratifs et des marchés. Le cadre réglementaire ne prévoit pas la possibilité dans un premier temps pour les opérateurs économiques insatisfaits ou mécontents d'une décision relative aux marchés publics de soumettre leurs plaintes à l'entité adjudicatrice. En effet cette approche permet d'éviter une action judicaire et peut être plus rapide et moins onéreuse (OCDE, 2011 [48]). L'Algérie devrait considérer la possibilité de prévoir le recours auprès de l'entité adjudicatrice afin de contester les décisions relevant du domaine des marchés publics avant de recourir à d'autres moyens ou organismes.

La commission des marchés est composée de plusieurs membres, y compris des représentants du service contractant, ce qui implique que l'indépendance de l'entité de recours n'est pas assurée (OCDE, 2017[4]). De plus, cette même commission est également compétente pour examiner les projets de cahiers des charges et les projets de marchés, ce qui signifie qu'en cas de recours elle doit revoir ses propres décisions. Afin d'améliorer l'efficacité du système et de renforcer la confiance du secteur privé dans le système de passation des marchés publics dans son ensemble, l'Algérie devrait réformer son système de recours en assurant l'indépendance totale de l'organisme de recours. L'ARMP ou toute entité indépendante de la procédure pourrait jouer ce rôle.

Par ailleurs, des litiges peuvent survenir durant l'exécution des contrats, Une section du DMP est prévue pour le règlement des litiges à l'amiable (articles 153-155). Cependant, d'après le DMP, dans le cas où le règlement du litige n'a pas abouti, une procédure différente est prévue pour les partenaires cocontractants étrangers et nationaux. En effet, en cas de désaccord, le litige est soumis à l'examen du comité de règlement amiable des litiges compétent pour les partenaires cocontractants nationaux alors que pour les partenaires cocontractants étrangers, le recours est examiné par l'organe national de règlement des litiges qui fait partie de l'ARMP et des délégations de service public. Ceci peut remettre en cause le traitement équitable des fournisseurs. Par ailleurs, les membres des commissions de règlement amiable des litiges sont essentiellement des représentants du service contractant ou du pouvoir exécutif, ce qui remet en question la partialité des décisions rendues. L'Algérie gagnerait donc à instaurer un système de règlement des litiges similaire pour tous les fournisseurs, et à assurer l'impartialité et l'indépendance totale des commissions de règlements des litiges, lesquelles pourraient être constituées d'experts techniques et juridiques indépendants. 


\section{Propositions d'action}

\begin{tabular}{|c|c|c|c|}
\hline Recommandations & $\begin{array}{l}\text { Court } \\
\text { terme }\end{array}$ & $\begin{array}{c}\text { Moyen } \\
\text { terme }\end{array}$ & $\begin{array}{l}\text { Long } \\
\text { terme }\end{array}$ \\
\hline $\begin{array}{l}\text { Développer une stratégie nationale avec des objectifs clairs en terme d'intégrité domaine des marchés } \\
\text { publics }\end{array}$ & & $\mathrm{X}$ & \\
\hline $\begin{array}{l}\text { Prendre en compte les marchés publics dans la stratégie nationale, ou bien développer une stratégie } \\
\text { spécifique pour les marchés publics cohérente avec la stratégie nationale }\end{array}$ & & $\mathrm{X}$ & \\
\hline $\begin{array}{l}\text { Mettre en place l'ARMP pour renforcer la stratégie et la politique de lutte contre la corruption dans les } \\
\text { marchés publics }\end{array}$ & $\mathrm{X}$ & & \\
\hline $\begin{array}{l}\text { Impliquer l'ensemble des parties prenantes pour le développement de la stratégie d'amélioration de l'intégrité } \\
\text { et de lutte contre la corruption, y compris dans le domaine des marchés publics }\end{array}$ & & $\mathrm{X}$ & \\
\hline $\begin{array}{l}\text { Publier les rapports et les recommandations de l'ONPLC et les avis du Conseil de veille et d'évaluation sur } \\
\text { leur site web et les partager avec l'ensemble des parties prenantes }\end{array}$ & & $\mathrm{X}$ & \\
\hline $\begin{array}{l}\text { En cas de dissolution définitive de l'OCRC et de la création du pôle pénal financier, s'assurer que les } \\
\text { activités du pôle financer reprennent au minimum celles de l'OCRC, qu'elles soient transparentes et } \\
\text { efficaces }\end{array}$ & $\mathrm{X}$ & & \\
\hline $\begin{array}{l}\text { Rendre publiques les informations sur les activités des autorités en charge de la lutte contre la corruption tout } \\
\text { en assurant la confidentialité lorsque l'instruction des dossiers l'exige }\end{array}$ & & & $\mathrm{X}$ \\
\hline $\begin{array}{l}\text { Assurer la coordination du pôle pénal financier avec les autorités impliquées dans le système de passation et } \\
\text { de contrôle des marchés publics }\end{array}$ & & $\mathrm{X}$ & \\
\hline $\begin{array}{l}\text { Développer et adopter le code d'éthique et de déontologie pour les agents publics impliqués dans les } \\
\text { marchés publics, les consulter pour assurer un « sentiment d'appropriation » }\end{array}$ & & $\mathrm{X}$ & \\
\hline Communiquer les valeurs d'éthique et de déontologie du secteur public au secteur privé & $X$ & & \\
\hline $\begin{array}{l}\text { Mener des actions de sensibilisation afin que les déclarations de probité des soumissionnaires ne soient pas } \\
\text { remplies comme un document administratif mais comme un document juridique }\end{array}$ & & $\mathrm{X}$ & \\
\hline $\begin{array}{l}\text { Garantir que la commission d'ouverture des plis et d'évaluation des offres s'assure que les fournisseurs ne } \\
\text { sont pas sur la liste de ceux exclus }\end{array}$ & & $\mathrm{X}$ & \\
\hline $\begin{array}{l}\text { Définir la notion de conflit d'intérêts dans les marchés publics dans son cadre réglementaire, et fournir des } \\
\text { explications supplémentaires au moyen de notes d'informations. }\end{array}$ & $\mathrm{X}$ & & \\
\hline Préciser dans le cadre réglementaire comment déclarer les conflits d'intérêt. & & & $\mathrm{X}$ \\
\hline Clarifier les dispositions relatives à la signature de déclaration d'absence de conflit d'intérêt & $\mathrm{X}$ & & \\
\hline Fournir une définition concrète du terme « modification substantielle de patrimoine » (loi n06-01) & & $\mathrm{X}$ & \\
\hline $\begin{array}{l}\text { Prendre des mesures afin d'élargir l'obligation de déclaration de patrimoine à tous les agents impliqués dans } \\
\text { les marchés publics }\end{array}$ & & & $x$ \\
\hline Développer et mettre en place une plateforme électronique pour la déclaration de patrimoine & & $\mathrm{X}$ & \\
\hline $\begin{array}{l}\text { Assurer la mise à jour effective de la liste d'exclusion des opérateurs économiques et s'assurer de sa } \\
\text { disponibilité en ligne }\end{array}$ & & $\mathrm{x}$ & \\
\hline $\begin{array}{l}\text { intégrer structurellement, dans toutes les actions de renforcement des capacités dans le domaine des } \\
\text { marchés publics, un module consacré aux normes et valeurs d'intégrité, ainsi qu'aux mécanismes de lutte } \\
\text { contre la corruption }\end{array}$ & & $\mathrm{X}$ & \\
\hline $\begin{array}{l}\text { Déployer le module de e-learning développé par l'ONPLC et l'ISGP et prévoir sa mise à jour au vu des } \\
\text { changements règlementaires en matière de lutte contre la corruption. }\end{array}$ & $\mathrm{X}$ & & \\
\hline $\begin{array}{l}\text { Assurer la participation des acteurs non publics dans le système tout au long du cycle de passation des } \\
\text { marchés publics afin d'assurer son intégrité et sa transparence }\end{array}$ & & $\mathrm{x}$ & \\
\hline $\begin{array}{l}\text { Mettre en place un système de signalement de cas d'atteinte à l'intégrité qui garantisse l'anonymat de } \\
\text { l'auteur du signalement }\end{array}$ & & & $\mathrm{X}$ \\
\hline Établir un système effectif des trois lignes de maitrise afin d'améliorer son système de contrôle interne & & & $\mathrm{X}$ \\
\hline $\begin{array}{l}\text { Rendre l'ouverture des plis électronique une fois le système électronique de passation des marchés publics } \\
\text { en place }\end{array}$ & & & $X$ \\
\hline $\begin{array}{l}\text { S'assurer de l'indépendance des membres de la Commission des marchés représentants le service } \\
\text { contractant pour continuer de qualifier le contrôle de cette commission comme « contrôle externe » dans le } \\
\text { cas contraire considérer cette commission comme un mécanisme de contrôle interne }\end{array}$ & $\mathrm{X}$ & & \\
\hline $\begin{array}{l}\text { Publier les rapports de l'IGF tout en respectant toutes les exigences de protection des données personnelles } \\
\text { ou confidentielles }\end{array}$ & & $\mathrm{X}$ & \\
\hline $\begin{array}{l}\text { Mettre à jour le logiciel d'audit de l'IGF pour prendre en compte le nouveau cadre réglementaire régissant les } \\
\text { marchés publics }\end{array}$ & $\mathrm{X}$ & & \\
\hline Publier les rapports de la Cour des comptes & & $X$ & \\
\hline
\end{tabular}




\begin{tabular}{l|c|c}
\hline Effectuer des audits de performance par les entités de contrôle (et en particulier la Cour des comptes) & $\mathrm{X}$ \\
\hline Établir un système de communication entre les différentes instances de contrôle & $\mathrm{X}$ \\
\hline $\begin{array}{l}\text { Mettre en place un système robuste de suivi des plans d'action pour mettre en œuvre les recommandations } \\
\text { des audits }\end{array}$ & $\mathrm{X}$ \\
\hline Développer une stratégie de management des risques dans les marchés publics & \\
\hline Assurer l'application du système de sanctions prévu dans le cadre réglementaire & $\mathrm{X}$ \\
\hline $\begin{array}{l}\text { Assurer l'analyse et la communication des statistiques relatives aux sanctions afin d'identifier les zones à } \\
\text { haut risque dans le domaine des marchés publics et améliorer le système de contrôle }\end{array}$ & $\mathrm{X}$ \\
\hline $\begin{array}{l}\text { Prévoir le recours auprès de l'entité adjudicatrice afin de contester ses décisions relevant du domaine des } \\
\text { marchés publics avant de recourir à d'autres moyens ou organismes }\end{array}$ & $\mathrm{X}$ \\
\hline Réformer le système de recours en assurant l'indépendance totale de l'organisme de recours & \\
\hline $\begin{array}{l}\text { Instaurer un système de règlement des litiges similaire pour tous les fournisseurs et assurer l'impartialité et } \\
\text { l'indépendance totale des commissions de règlement des litiges }\end{array}$ & $\mathrm{X}$ \\
\hline
\end{tabular}

Note : Court terme : recommandation à mettre en application dans les 12 mois suivants;

Moyen terme : recommandation à mettre en application dans les 1 - 2 ans ;

Long terme : recommandation à mettre en application dans plus de 2 ans. 


\section{Références}

AFA (Agence Française Anticorruption) (2018), Rapport annuel d'activité 2017, https://www.economie.gouv.fr/files/files/directions services/afa/Rapport annuel 22 mai 2018.pdf (consulté le 29 janvier 2019).

ALG24 (s.d.), Corruption : Un pôle pénal financier pour remplacer l'OCRC, https://www.alg24.net/exclusif-corruption-pole-penal-financier-remplacer-locrc/ (consulté le 29 janvier 2019).

Algérie Presse Service (s.d.), Patrimoine: 54.000 déclarations d'élus locaux et plus de 6000 de hauts fonctionnaires, http://www.aps.dz/algerie/81931-54-000-declarations-de-patrimoinedeposees-par-des-elus-locaux-et-plus-de-6000-par-des-hauts-fonctionnaires (consulté le 12 février 2019).

Ambassade des États-Unis en Algérie (2016), Rapport 2016 sur les Droits de l'Homme - Algérie, https://dz.usembassy.gov/wp-content/uploads/sites/236/ALGERIA-HRR-2016-FRE-FINAL.pdf (consulté le 30 janvier 2019).

Commission Européenne (2014), Report from the Commission to the Council and the European Parliament; EU Anti-Corruption Report, http://eurlex.europa.eu/LexUriServ/LexUriServ.do?uri=COM:2011:0308:FIN:EN:PDF (consulté le 22 janvier 2019).

Conseil de l'Europe (2013), Designing and Implementing Anti-corruption Policies: Handbook, https://rm.coe.int/16806d8ad7 (consulté le 28 janvier 2019).

Conseil des Communautés Européennes (1989), Directive du Conseil du 21 décembre 1989 portant coordination des dispositions législatives, réglementaires et administratives relatives à l'application des procédures de recours en matière de passation des marchés publics de fournitures et de travaux, https://eur-lex.europa.eu/legalcontent/FR/TXT/PDF/?uri=CELEX:01989L0665-20140417\&from=EN (consulté le 31 janvier 2019).

COSO (2013), Internal Control-Integrated framework, https://www.coso.org/Documents/990025P-Executive-Summary-final-may20.pdf (consulté le 14 février 2019).

de Koker, L. et K. Harwood (2015), « Supply Integrity Due Diligence in Public Procurement: Limiting the Criminal Risk to Australia », Sydney Law Review, vol. 11, http://www.austlii.edu.au/au/journals/SydLRev/2015/11.html (consulté le 5 juillet 2019).

Gouvernement du Canada (s.d.), Le régime d'intégrité du gouvernement du Canada, 2018, https://www.tpsgc-pwgsc.gc.ca/ci-if/ci-if-fra.html (consulté le 20 janvier 2019).

HAICOP (2019), Stratégie de Management des Risques dans les Marchés Publics en Tunisie, https://www.oecd.org/gov/public-procurement/publications/strat\%C3\%A9gie-managementdes-risques-march\%C3\%A9s-publics-tunisie.pdf (consulté le 11 juin 2019).

HM Government (2017), United Kingdom Anti-Corruption Strategy 2017-2022, https://www.gov.uk/government/publications/uk-anti-corruption-strategy-2017-to-2022 (consulté le 29 janvier 2019). 
IIA (2013), Les trois lignes de maîtrise pour une gestion des risques et un contrôle efficaces, https://na.theiia.org/translations/PublicDocuments/PP\%20The\%20Three\%20Lines\%20of\%20 Defense\%20in\%20Effective\%20Risk\%20Management\%20and\%20Control\%20French.pdf (consulté le 14 février 2019).

Inspection Générale des Finances, France (s.d.), Rapports publics, http://www.igf.finances.gouv.fr/cms/sites/igf/accueil/rapports-publics.html (consulté le 30 janvier 2019).

INTOSAI (2004), Guidelines for Internal Control Standards for the Public Sector, http://www.intosai.org (consulté le 15 février 2019).

ISO (2018), ISO 31000:2018: Management du risque Lignes directrices, https://www.iso.org/fr/standard/65694.html (consulté le 12 septembre 2018).

Liberté Algérie (s.d.), Vers la création d'un pôle pénal financier, 06/02/2019, https://www.libertealgerie.com/actualite/vers-la-creation-dun-pole-penal-financier-308935 (consulté le 13 février 2019).

Ministère des Finances, Algérie (2011), Evolution du cadre juridique de l'IGF, http://www.mf.gov.dz/article/72/Inspection--G\%C3\%A9n\%C3\%A9rale-desFinances/523/Evolution-du-cadre-juridique-de-l'IGF.html (consulté le 14 février 2019).

New Zealand Government Procurement and Property (s.d.), Managing conflicts of interest and confidentiality, https://www.procurement.govt.nz/procurement/guide-to-procurement/planyour-procurement/managing-conflicts-of-interest-and-confidentiality/ (consulté le 29 janvier 2019).

OCDE (2019), OECD Integrity Review of Argentina: Achieving Systemic and Sustained Change, Examens de l'OCDE sur la gouvernance publique, Éditions OCDE, Paris, https://dx.doi.org/10.1787/g2g98ec3-en.

OCDE (2019), OECD Integrity Review of Mexico City: Upgrading the Local Anti-corruption System, Examens de l'OCDE sur la gouvernance publique, Éditions OCDE, Paris, https://dx.doi.org/10.1787/9789264306547-en.

OCDE (2018), Diagnostic d'intégrité au Maroc : Mettre en œuvre des politiques d'intégrité pour renforcer la confiance, Examens de l'OCDE sur la gouvernance publique, Éditions OCDE, Paris, https://dx.doi.org/10.1787/9789264302693-fr.

OCDE (2018), Procurement Review of the Chamber of Commerce of Bogotá, Colombia: Aligning Practices with the OECD Public Procurement Recommendation, OCDE, Paris, https://www.oecd.org/gov/public-procurement/publications/procurement-review-chambercommerce-bogota.pdf.

OCDE (2018), Public Procurement in Nuevo León, Mexico : Promoting Efficiency through Centralisation and Professionalisation, Examens de l'OCDE sur la gouvernance publique, Éditions OCDE, Paris, https://dx.doi.org/10.1787/9789264288225-en.

OCDE (2017), OECD Integrity Review of Mexico: Taking a Stronger Stance Against Corruption, Examens de l'OCDE sur la gouvernance publique, Éditions OCDE, Paris, https://dx.doi.org/10.1787/9789264273207-en. 
OCDE (2017), OECD Integrity Review of Peru: Enhancing Public Sector Integrity for Inclusive Growth, Examens de l'OCDE sur la gouvernance publique, Éditions OCDE, Paris, https://dx.doi.org/10.1787/9789264271029-en.

OCDE (2017), Panorama des administrations publiques 2017, Éditions OCDE, Paris, http://dx.doi.org/10.1787/gov glance-2017-fr.

OCDE (2017), Recommandation du Conseil de l'OCDE sur l'intégrité publique, OCDE, Paris, http://www.oecd.org/gov/ethics/Recommandation-integrite-publique.pdf (consulté le 20 juillet 2018).

OCDE (2016), Le contrôle interne et la gestion des risques pour renforcer la gouvernance en Tunisie, OCDE, Paris, https://www.oecd.org/mena/governance/Controle interne et la gestion des risques en Tu nisie.pdf (consulté le 30 janvier 2019).

OCDE (2016), Le gouvernement ouvert en Tunisie, Examens de l'OCDE sur la gouvernance publique, Éditions OCDE, Paris, https://dx.doi.org/10.1787/9789264227170-fr.

OCDE (2016), Prévention de la corruption dans les marchés publics, https://www.oecd.org/gov/public-procurement/publications/prevention-corruption-marchespublics.pdf (consulté le 28 décembre 2018).

OCDE (2015), Recommandation du Conseil de l'OCDE sur les marchés publics, OCDE, Paris, http://www.oecd.org/fr/gov/ethique/Recommandation-OCDE-sur-les-marches-publics.pdf (consulté le 16 août 2018).

OCDE (2013), «Establishing Procurement Review Bodies », SIGMA Public Procurement Briefs, $\mathrm{n}^{\circ} 25$, Éditions OCDE, Paris, https://dx.doi.org/10.1787/5js4vmn47gzr-en.

OCDE (2012), Recommandation du conseil de l'OCDE sur la lutte contre les soumissions concertées dans les marchés publics, OCDE, Paris, http://www.oecd.org (consulté le 19 juillet 2018).

OCDE (2011), Principes de l'OCDE pour renforcer l'intégrité dans les marchés publics, Éditions OCDE, Paris, https://dx.doi.org/10.1787/9789264056626-fr.

OCDE (2011), « Remedies », SIGMA Public Procurement Briefs, $n^{\circ} 12$, Éditions OCDE, Paris, https://dx.doi.org/10.1787/5js4wzv2k2vl-en.

OCDE (2006), Gérer les conflits d'intérêts dans le secteur public : Mode d'emploi, Éditions OCDE, Paris, https://dx.doi.org/10.1787/9789264018259-fr.

OCDE (2003), Recommandation du Conseil sur les lignes directrices pour la gestion des conflits d'intérêts dans le service public, OCDE, Paris, http://www.oecd.org/gov/ethics/49106150.pdf (consulté le 29 janvier 2019).

OCDE (s.d.), « Assessment of Anti-Corruption Reforms in Public Procurement, Health, and Tax \& Customs $»$.

Olivieri, C. et al. (2015), « Control and Public Management Performance in Brazil: Challenges for Coordination », International Business Research, vol. 8/8, http://dx.doi.org/10.5539/ibr.v8n8p181. 
OMC (Organisation Mondiale du Commerce) (s.d.), Accord revisé sur les marchés publics, https://www.wto.org/french/docs f/legal f/rev-gpr-94 01 f.htm (consulté le 31 janvier 2019).

ONPLC (2019), Formation anti-corruption à Tamenghest, http://onplc.org.dz/index.php/fr/?view=article\&id=151:formation-anti-corruption-atamenghest\&catid=24:actualites (consulté le 12 février 2019).

ONPLC (s.d.), Bilan de l'activité déclarations de patrimoine au 30 septembre - 2015, http://www.onplc.org.dz/index.php/fr/documents/documents-5 (consulté le 12 février 2019).

ONPLC (s.d.), Projet de code d'éthique et de déontologie des agents publics chargés de la passation des marchés publics, http://www.onplc.org.dz/images/ONPLC/fran\%C3\%A7ais/codes\%20dontologie.pdf (consulté le 12 février 2019).

ONPLC (s.d.), Statut juridique, Missions et Organisation de l'ONPLC, http://www.onplc.org.dz/images/ONPLC/fran\%C3\%A7ais/D\%C3\%A9pliant fonctionement O NPLC.pdf (consulté le 10 février 2019).

Services publics et Approvisionnement Canada (SPAC) (2018), Code de conduite de Services publics et Approvisionnement Canada, https://www.tpsgc-pwgsc.gc.ca/apropos-about/codecond-fra.html (consulté le 13 décembre 2018).

Transparency International (2018), L'indice de perception de la corruption 2017 met en avant l'ampleur du fardeau que représente la corruption dans plus des deux-tiers des pays $d u$ monde, https://www.transparency.org/news/pressrelease/lindice de perception de la corruption 20 17 met en avant lampleur du fardea (consulté le 12 décembre 2018).

UNDP (2014), Anti-corruption Strategies: Understanding what works, what doesn't and why?, http://www.undp.org/content/dam/undp/library/Democratic\%20Governance/Anticorruption/UNDP\%20ACS\%20Asia\%20Pacific\%20\%20Anti-corruption\%20Strategies.pdf (consulté le 25 janvier 2019).

UNODC (2015), Rapport d'examen de l'Algérie, https://www.unodc.org/documents/treaties/UNCAC/CountryVisitFinalReports/2015 $0803 \quad \mathrm{Al}$ geria Final Country Report French.pdf (consulté le 28 janvier 2019).

UNODC (2004), Convention des Nations Unies contre la Corruption, https://www.unodc.org/res/ii/import/international standards/united nations convention again st corruption/uncac french.pdf (consulté le 31 janvier 2019).

Vargas, G. et D. Schlutz (2016), « Opening Public Officials' Coffers: A Quantitative Analysis of the Impact of Financial Disclosure Regulation on National Corruption Levels », European Journal on Criminal Policy and Research, vol. 22/3, pp. 439-475, http://dx.doi.org/10.1007/s10610-016-9320-3.

World Economic Forum (2015), The Global Competitiveness Report, [6] http://www3.weforum.org/docs/gcr/2015-2016/Global Competitiveness Report 20152016.pdf (consulté le 13 juin 2019). 


\section{Transparence et dématérialisation du système de passation des marchés publics en Algérie}

Améliorer la transparence tout au long du cycle de passation des marchés publics en Algérie est un enjeu majeur pour assurer l'efficacité et la bonne gouvernance du système. Par ailleurs, le pays ne possède pas encore de système de passation électronique des marchés publics. Ce chapitre dresse un état des lieux et fournit des recommandations aux parties prenantes pour renforcer la transparence du système algérien de passation des marchés publics. II souligne également la nécessité pour l'Algérie de développer une stratégie pour la mise en place effective du système de passation électronique des marchés publics (e-procurement). 
La transparence est essentielle pour le bon fonctionnement du système de passation des marchés publics; il s'agit également d'un principe fondamental de la Recommandation du Conseil de l'OCDE sur les marchés publics (OCDE, 2015[1] $)$ mais aussi de la Recommandation du Conseil de l'OCDE sur l'intégrité publique (OCDE, 2017 $\left.{ }_{[2]}\right)$. En effet, elle permet d'une part à l'ensemble des parties prenantes, y compris les fournisseurs potentiels nationaux et étrangers, la société civile et le grand public, d'accéder gratuitement à l'information relative aux marchés publics, d'une part, de réduire les risques inhérents aux marchés publics et de responsabiliser les différents acteurs, d'autre part. Par ailleurs, la transparence dans le processus de passation des marchés publics garantit le traitement juste et équitable des fournisseurs potentiels et la fourniture d'informations nécessaires aux citoyens et aux différentes parties prenantes sur la façon dont l'État dépense l'argent du contribuable.

Les systèmes de passation électronique des marchés publics améliorent la transparence, mais aussi l'efficience et l'accès aux marchés publics, en particulier pour les petites et moyennes entreprises (PME) (EBRD, 2015[3]). L'Algérie est l'un des seuls pays de la région Moyen-Orient et Afrique du Nord (MENA) à ne pas disposer d'un système de passation électronique des marchés publics, mais le pays est en cours d'acquisition d'une plateforme de dématérialisation des marchés publics.

Ce chapitre analyse le niveau de transparence et de numérisation du système de passation des marchés publics en Algérie et fournit des recommandations conformes aux les meilleures pratiques internationales.

\subsection{Assurer un degré adéquat de transparence dans le système de passation des marchés publics}

Le principe de transparence est un élément central d'un système de passation des marchés publics efficient et effectif. L'encadré 3.1 indique les liens étroits entre transparence et d'autres principes de la Recommandation du Conseil de l'OCDE sur les marchés publics.

Encadré 3.1. Liens entre la transparence et les autres principes de la Recommandation du Conseil de l'OCDE sur les marchés publics

- Intégrité : la divulgation d'information sur les processus des marchés publics contribue à identifier et diminuer les cas de mauvaise gestion, de fraude et de corruption.

- Accès : la transparence contribue à traiter équitablement les fournisseurs potentiels et à améliorer leur accès aux opportunités de marchés publics.

- Participation : la transparence permet de fournir des informations sur le système de passation des marchés publics aux fournisseurs potentiels (nationaux et internationaux), la société civile, et le grand public. La mise en place d'actions inclusives lors de changements réglementaires du système de passation des marchés publics par des consultations publiques augmente la transparence du système.

- Efficience : la transparence permet aux décideurs politiques d'organiser et de structurer les marchés stratégiquement et d'améliorer leur efficience.

- Systèmes électroniques de passation des marchés publics : Ils renforcent la transparence en mettant à disposition des informations sur les processus des marchés publics.

- Reddition des comptes : la transparence favorise la reddition des comptes en rendant compte des processus de passation des marchés publics et de la dépense publique.

- Intégration : assurer la visibilité des fonds publics, dès le début de la budgétisation et tout au long du processus de passation des marchés publics.

Source : (OCDE, 2016[4]). 
La transparence est un principe fondamental du cadre réglementaire régissant les marchés publics de la majorité des États. C'est le cas en Algérie, où l'article 5 du décret présidentiel n 15-247 de 2015 portant réglementation des marchés publics et des délégations de service public (DMP) mentionne " qu'en vue d'assurer l'efficacité de la commande publique et la bonne utilisation des fonds publics, les marchés publics doivent respecter les principes de liberté d'accès à la commande publique, d'égalité de traitement des candidats, et de transparence des procédures ».

La transparence ne se limite pas à la publication des informations et documents relatifs aux marchés publics, tels que le cadre réglementaire, les informations sur les procédures d'appels d'offres et les marchés (passés et à venir), les flux financiers et les budgets des différentes entités. II s'agit également d'adapter les moyens de communication et l'information aux différents utilisateurs et parties prenantes.

\subsubsection{Améliorer la publication d'informations sur les procédures de passation des marchés publics tout au long du cycle de passation des marchés publics}

\section{Publier plus d'informations et documents sur les procédures de passation des marchés publics}

La Recommandation du Conseil de l'OCDE sur les marchés publics souligne la nécessité de publier un certain nombre d'informations et documents tout au long du cycle de passation des marchés publics (OCDE, 2015 [1] $)$. En effet, les pays font plus d'efforts de transparence durant les phases de pré-soumission et de soumission, mais les risques de mauvaise gestion, d'inefficacité ou d'atteinte à l'intégrité existent tout autant lors de la phase de gestion contractuelle (Transparency International, 2014 ${ }_{[5]}$ ).

En Algérie, le cadre réglementaire prévoit la publication et la transmission d'un certain nombre de documents et d'informations relatives aux marchés publics à toutes ou certaines parties prenantes (voir tableau 3.1).

Tableau 3.1. Obligation de transparence lors des procédures de passation des marchés publics en Algérie

\begin{tabular}{|c|c|c|c|}
\hline $\begin{array}{l}\text { Phases de } \\
\text { passation des } \\
\text { marchés publics }\end{array}$ & Documents ou informations & $\begin{array}{l}\text { Obligation de transparence } \\
\text { Oui /Non }\end{array}$ & $\begin{array}{c}\text { Parties prenantes auxquelles les } \\
\text { documents et informations } \\
\text { doivent être fournis }\end{array}$ \\
\hline \multirow{4}{*}{$\begin{array}{l}\text { Phase de pré- } \\
\text { soumission }\end{array}$} & Programme prévisionnels & OUI & $\begin{array}{l}\text { Toutes les parties prenantes } \\
\text { (si publié sur le site web de l'entité) }\end{array}$ \\
\hline & $\begin{array}{l}\text { Liste des opérateurs } \\
\text { économiques exclus/ interdits } \\
\text { de la participation aux marchés } \\
\text { publics }\end{array}$ & OUI & $\begin{array}{l}\text { Toutes les parties prenantes (publié } \\
\text { sur le site du ministère des Finances) }\end{array}$ \\
\hline & Disponibilité du budget & NON & \\
\hline & Avis d'information préalable & NON & \\
\hline \multirow{6}{*}{$\begin{array}{l}\text { Phase de } \\
\text { soumission et } \\
\text { d'adjudication }\end{array}$} & Avis d'appel d'offres & OUI & $\begin{array}{l}\text { À toutes les parties prenantes (si } \\
\text { publié sur le site web de l'entité ou } \\
\text { dans la presse) }\end{array}$ \\
\hline & Dossier d'appel d'offres & OUI & $\begin{array}{l}\text { Uniquement soumissionnaires, qui } \\
\text { doivent le retirer auprès du service } \\
\text { contractant }\end{array}$ \\
\hline & $\begin{array}{l}\text { Éclaircissements relatifs aux } \\
\text { questions des soumissionnaires }\end{array}$ & $\begin{array}{l}\text { OUI } \\
\text { (uniquement pour les } \\
\text { soumissionnaires) }\end{array}$ & Uniquement les soumissionnaires \\
\hline & Avis d'attribution provisoire & OUI & $\begin{array}{l}\text { Toutes les parties prenantes (publié } \\
\text { dans la presse) }\end{array}$ \\
\hline & Avis de notification & NON & \\
\hline & Décision sur les appels/recours & NON & \\
\hline
\end{tabular}




\begin{tabular}{l|l|l|l}
\hline & Contrat signé & NON \\
\hline \multirow{4}{*}{\begin{tabular}{l}
\multirow{2}{*}{$\begin{array}{l}\text { Phase de gestion } \\
\text { contractuelle }\end{array}$} \\
\cline { 2 - 3 }
\end{tabular}} & Variations/avenants & NON & \\
\cline { 2 - 3 } & Calendrier et grandes étapes & NON \\
\cline { 2 - 3 } & Certificat d'achèvement & NON \\
\cline { 2 - 3 } & Audit final & NON \\
\hline
\end{tabular}

Source : DMP.

Le cadre réglementaire prévoit des bonnes pratiques, telles que la publication des programmes prévisionnels des projets à lancer durant l'exercice considéré ou la publication de la liste des opérateurs économiques exclus ou interdits de participation aux marchés publics. Cependant, la transparence pourrait être améliorée lors de toutes les phases de passation des marchés publics.

Pour la phase de pré-soumission, le cadre réglementaire algérien ne prévoit pas la publication d'avis de pré-information. II s'agit pourtant d'un élément nécessaire pour améliorer la transparence et l'accès des opérateurs économiques aux opportunités d'achat. Le gouvernement algérien devrait donc également prévoir dans son cadre réglementaire la possibilité pour les entités publiques de publier ce type d'avis.

Par ailleurs, le degré de transparence lors de la phase de gestion contractuelle est limité car le cadre réglementaire ne prévoit aucune obligation de transparence lors de cette phase. Par exemple, les différentes parties prenantes n'ont aucun moyen de savoir si un contrat a fait l'objet d'un avenant ou si un marché a été réalisé dans les délais et au budget prévus. C'est le cas également des rapports des organes de contrôle. En effet, les rapports de l'Inspection générale des finances (IGF) et de la Cour des comptes, qui contiennent un volet marchés publics, ne sont pas disponibles en ligne, ce qui n'est pas considéré comme une bonne pratique internationale. Le gouvernement algérien gagnerait donc à publier les informations relatives à la gestion contractuelle, ainsi que les rapports des organes de contrôle externes a posteriori.

\section{S’assurer du respect des obligations de transparence dans les marchés publics}

Prévoir des obligations de transparence dans le cadre réglementaire est essentiel pour améliorer l'accès des opérateurs économiques aux marchés publics, mais également pour renforcer la confiance des citoyens. II est également nécessaire de s'assurer du respect de ces obligations de transparence (OCDE, 2017[6]).

D'après les recherches de l'équipe de l'OCDE, certains documents dont la publication est obligatoire selon la réglementation ne sont pas toujours publiés. C'est le cas par exemple des programmes prévisionnels et de la liste des opérateurs économiques exclus/interdits de la participation aux marchés publics. Les programmes prévisionnels ne sont publiés ni sur le Bulletin officiel des marchés de l'opérateur public (Bomop), ni sur le site web des entités publiques. Par ailleurs, il n'a pas été possible de trouver la liste des opérateurs exclus de la participation aux marchés publics. Lors de la mission exploratoire, plusieurs entités publiques du niveau central et régional, telles que le ministère de l'Habitat, ont indiqué détenir une liste des opérateurs exclus des marchés publics. Cependant ces listes ne sont pas publiées et centralisées comme indiqué dans le cadre réglementaire. Le gouvernement algérien devrait donc s'assurer de l'obligation du respect des obligations de transparence dans les marchés publics.

\subsubsection{Assurer la mise en place de moyens de communication effectifs permettant la publication d'informations sur les marchés}

Assurer la transparence dans le domaine des marchés publics nécessite de mettre en place des moyens de publication adéquats, faciles d'accès, gratuits et accessibles à tous (OCDE, 2015 ${ }_{[1]}$ ). La majorité des pays membres et non-membres de l'OCDE publient les informations sur un portail national en plus d'autres moyens de publication tels que les journaux nationaux et les locaux des entités publics. En Algérie, 
actuellement quatre moyens de publication sont à la disposition des entités publiques : le Bomop géré par l'Agence nationale d'édition et de publication (ANEP), la presse, les sites web et les locaux de l'entité publique. Le cadre réglementaire mentionne également le portail des marchés publics, cependant ce dernier n'a pas encore été mis en place.

\section{Assurer la gratuité et l'accès de toutes les parties prenantes aux informations sur les marchés publics contenues dans le journal officiel}

Le Bomop est actuellement le moyen de publication qui centralise des informations clé du système de passation des marchés publics. II existe sous format papier mais également sous format dématérialisé. Le format dématérialisé facilite l'accès des opérateurs économiques aux opportunités d'achat. D'après le cadre réglementaire, les entités publiques sont tenues de publier sur le Bomop les programmes prévisionnels des projets à lancer durant l'exercice considéré et la liste des marchés publics conclus durant l'exercice précédent (art. 158 du DMP) ; les avis d'appel d'offres (art. 65 du DMP); et certains indices de prix (art. 103 du DMP). Cependant, contrairement à la pratique de plusieurs pays, y compris ceux de l'OCDE, l'accès au Bomop n'est pas gratuit. Le coût annuel de l'abonnement au support papier est fixé à 15280 DZD (dinars algériens), la consultation en ligne à 12224 DZD, l'option permettant la consultation papier et en ligne est fixée à 27505 DZD (soit, respectivement,113 EUR [euros], 90 EUR et 204 EUR). Le fait que ces informations ne soient pas disponibles gratuitement et que le coût de l'abonnement au Bomop soit relativement élevé entravent fortement l'accès des opérateurs économiques aux opportunités d'achat, mais également l'accès de la société civile et du grand public aux informations sur les marchés publics. En France, par exemple, le Bulletin officiel des annonces des marchés publics(BOAMP) est gratuit et accessible à toutes les parties prenantes (Direction de l'information légale et administrative (DILA), France, s.d.[7]).

Par ailleurs, la navigation sur le site du Bomop manque de clarté, les différents documents devant être publiés ne sont pas organisés dans des rubriques spécifiques telles que les indices de prix et les programmes prévisionnels. De plus, l'outil de recherche du Bomop propose des résultats d'une pertinence limitée. Lors des missions exploratoires de l'OCDE, certaines parties prenantes ont aussi fait part des retards et de décalages dans la publication du Bomop.

Le gouvernement algérien devrait assurer à toutes les parties prenantes un accès gratuit et instantané aux informations publiées sur le Bomop et améliorer l'organisation du site web afin de faciliter l'accès à l'information sur les marchés publics. Par ailleurs, le portail électronique des marchés publics qui est en cours de développement prévoit également la publication de documents relatifs aux marchés publics, y compris les avis d'appel d'offres. Le gouvernement algérien devra donc décider de maintenir ou non l'obligation de publication sur le Bomop.

\section{Doter toutes les entités publiques algériennes de sites web et y publier les informations relatives aux marchés publics}

En plus d'un portail centralisé et des bulletins officiels, dans plusieurs pays de l'OCDE les entités publiques publient des informations sur leurs activités dans le domaine des marchés publics sur leur site web. Ceci est un moyen supplémentaire permettant aux opérateurs économiques d'accéder aux opportunités d'achat de l'entité publique, en particulier pour les opportunités en dessous des seuils d'appel d'offres. Par ailleurs cela permet aux différentes parties prenantes, y compris aux citoyens, d'observer comment l'argent public géré par l'entité en question est dépensé, en particulier pour les pays qui ne sont pas dotés d'un portail centralisant les informations relatives aux marchés publics.

En Algérie, le cadre réglementaire prévoit la publication d'informations relatives aux marchés publics sur le site web des entités publiques (liste des marchés conclus et noms des entreprises attributaires, programme prévisionnel des projets à lancer durant l'exercice). Cependant d'après les recherches de l'équipe OCDE, les entités publiques ne disposent pas toutes de sites web. De plus, parmi celles qui en 
possèdent un, certaines ne publient pas d'informations sur les marchés publics et d'autres publient des informations incomplètes et non mises à jour. Le gouvernement algérien devrait donc doter toutes les entités publiques d'un site web et s'assurer de la publication des informations relatives aux marchés publics. Ceci pourrait être facilité par la création d'une interface entre les sites des différentes entités, le Bomop et le portail électronique des marchés publics à venir.

\subsubsection{Mettre à disposition des parties prenantes des informations sur le système de passation des marchés publics}

\section{Assurer la disponibilité des textes réglementaires, des politiques et des stratégies relatives aux marchés publics}

La connaissance du cadre réglementaire régissant les marchés publics est une obligation et elle est nécessaire à la bonne conduite des procédures de passation des marchés publics. Pour cela, il est primordial que toutes les parties prenantes puissent accéder facilement à ce cadre réglementaire (OCDE, 2015[1]).

En Algérie, une page du site du ministère des Finances est dédiée au cadre réglementaire régissant les marchés publics, mais elle n'inclut pas tous les arrêtés, décrets, politiques ou autres documents relatifs au système de passation des marchés publics (Ministère des Finances, Algérie, s.d. $\left[{ }_{0]}\right]$ ).Par ailleurs, le système de passation des marchés publics regroupant divers parties prenantes, la page web relative au cadre réglementaire régissant les marchés publics devrait contenir des liens vers les sites ou documents et politiques relatifs aux activités de ces parties prenantes (site du conseil de la concurrence ou de l'Organe national de prévention et de lutte contre la corruption [ONPLC], par exemple).II est donc nécessaire que le gouvernement algérien mette à disposition des différentes parties prenantes le cadre réglementaire régissant les marchés publics dans sa totalité sur une page web spécifique.

Par ailleurs, l'Algérie dispose d'un journal officiel où sont publiés tous les textes juridiques et réglementaires (le Journal officiel de la république algérienne démocratique et populaire [JORADP]). La version en ligne de ce journal est gérée par le Secrétariat général du gouvernement. Cependant, l'option de recherche ne semble pas fonctionner, ce qui empêche les parties prenantes d'accéder aux différentes lois, décrets ou autres documents juridiques (Secrétariat général du gouvernement, Algérie, s.d.[0]). Le secrétariat général du gouvernement gagnerait donc à améliorer la qualité du site web du JORADP afin d'améliorer l'accès au cadre réglementaire des différentes parties prenantes.

\section{La transparence dans les marchés publics passe aussi par l'accès aux informations sur la performance du système}

La transparence ne se limite pas à la publication de documents relatifs aux procédures de passation des marchés publics, elle nécessite également de rendre accessibles aux différentes parties prenantes des informations sur la performance globale du système (OCDE, 2015 $5_{[1]}$ ). Ces informations comprennent notamment celles relatives aux montants, aux catégories d'achat ou encore aux différentes typologies de procédures utilisées par les entités publiques. Ces données agrégées permettent de fournir une meilleure information aux contribuables et aux opérateurs économiques, mais également au gouvernement et aux entités publiques afin de prendre des décisions avisées quant aux différentes politiques et activités relatives aux marchés publics. L'encadré 3.2 présente l'exemple des informations publiées au Pérou sur la performance du système de passation des marchés publics. 


\section{Encadré 3.2. Informations sur la performance du système de passation des marchés publics au Pérou}

L'organisme supervisant les marchés publics de l'État péruvien (Organismo Supervisor de las Contrataciones del Estado [OSCE]) a mis en place un système de veille économique appelé Conosce pour fournir des informations de qualité sur les marchés publics. Conosce propose des tableaux de bord interactifs permettant de rechercher des statistiques sur les marchés publics de l'État attribués à des fournisseurs individuels, des groupements d'entreprise et des entités publiques. Les tableaux de bord contiennent également les informations suivantes :

- Des indicateurs sur les marchés publics de l'État : ces indicateurs incluent les montants des contrats attribués par région, par objet contractuel et par type de procédure, et le pourcentage de procédures annulées, infructueuses ou sans suite.

- Les fournisseurs de l'État: ces indicateurs incluent les montants alloués aux fournisseurs, individuellement ou en groupements. II est également possible d'effectuer des recherches spécifiques sur les contrats attribués à un fournisseur donné.

- Des informations et des indicateurs sur les marchés passés par chaque entité publique : le montant et le nombre de marchés attribués par chaque entité publique.

Ces informations sont publiées mensuellement et annuellement.

Source : (Organismo Supervisor de las Contrataciones del Estado (OSCE)- Perou, s.d.[10]).

En Algérie, il n'existe ni indicateurs ni statistiques sur les marchés publics (chapitre 4), ce qui empêche l'analyse de la performance du système. Cette situation est liée aux avancées limitées du pays dans le domaine de la dématérialisation des marchés publics, mais aussi à l'absence de mise en place de l'Autorité de régulation des marchés publics et des délégations de service public(ARMP) dont une des prérogatives est d'effectuer un recensement économique annuel de la commande publique (voir chapitre 1).Le gouvernement algérien devrait donc mettre en place un système de collecte de données visant à fournir des informations à toutes les parties prenantes sur la performance du système de passation des marchés publics.

\section{Prendre en compte le caractère confidentiel lors de la publication d'informations relatives aux marchés publics}

La publication des informations sur les marchés publics dépend également de considérations liées à la confidentialité de l'information. Certaines informations ne peuvent être divulguées à certaines parties prenantes pour plusieurs raisons (sécurité nationale, secret commercial de certains opérateurs économiques, etc.). En Norvège comme dans les pays de l'Union européenne (UE), la qualification du caractère confidentiel de l'information est l'un des enjeux rencontrés par les agents en charge des marchés publics $\left(\mathrm{OCDE}\right.$, à paraître $\left.{ }_{[11]}\right)$. En Algérie, le caractère confidentiel d'une procédure ou d'une information est arrêté au cas par cas, par décision du ministre, après avis de la commission sectorielle des marchés compétente. Cependant, il n'existe pas de doctrine claire permettant aux acheteurs publics d'apprécier la confidentialité de l'information. L'encadré 3.3 décrit les éléments principaux à prendre en compte dans les politiques de confidentialité.

Au Royaume-Uni, par exemple, la loi sur l'accès à l'information (Freedom of Information Act) mentionne des cas spécifiques où il est possible de ne pas publier l'information (menace de la sécurité de l'État, des 
individus, de la défense nationale, etc). Le Royaume-Uni a développé des lignes directrices relatives à la transparence dans les marchés publics (Crown Commercial Service, 2017[12]).

La définition de la notion de confidentialité influence les exigences de transparence, et donc les procédures à suivre. Ainsi, le gré à gré est favorisé lorsque les informations contenues dans le cahier des charges sont hautement confidentielles, la procédure restreinte avec la signature d'un accord de confidentialité permet de protéger les informations confidentielles, et enfin l'appel d'offres ouvert permet de ne fournir l'information confidentielle qu'à l'opérateur économique retenu (OCDE, à paraître [11]).

Le gouvernement algérien gagnerait donc à mettre en place une politique permettant aux acheteurs publics d'apprécier la confidentialité de l'information afin de pouvoir la publier le cas échéant. Cet aspect devra également être abordé dans le code d'éthique et de déontologie des agents publics intervenant dans le contrôle, la passation et l'exécution des marchés publics et des délégations de service public (voir section 2.2.1).

\section{Encadré 3.3. Les éléments principaux à prendre en compte dans les politiques de confidentialité}

- Définir des critères clairs permettant de préciser quelles sont les informations publiques, confidentielles ou sensibles.

- Mettre en place des lignes directrices pour les entités publiques afin de traiter les informations de nature commerciale, personnelle et financière à caractère sensible et confidentiel qu'elles reçoivent.

- Empêcher l'accès aux informations confidentielles qui permettent de détecter la déviation d'un accord collusoire et de faciliter les ententes lors de futures marchés.

- Établir des protocoles de sécurité afin de protéger les documents (physiques ou électroniques).

Source : (OCDE, 2016[4] $)$.

\subsection{Développer un système de passation électronique des marchés publics : un élément clé pour améliorer l'efficience du système}

Les systèmes de passation électronique des marchés publics (e-procurement) remplacent ou redéfinissent les procédures sur support papier grâce à l'intégration de technologies de l'information et des communications (OCDE, 2015 $[1]$ ). Les avantages de ces systèmes sont nombreux (encadré 3.4). 


\section{Encadré 3.4. Avantages des systèmes de passation électronique des marchés publics}

Avantages tangibles :

- $\quad$ prix plus bas, diminution des dépenses publiques

- procédures plus rapides

- coûts administratifs réduits

- standardisation des procédures

Avantages intangibles:

- meilleur accès des entreprises aux marchés publics

- plus de concurrence

- amélioration de la transparence

- amélioration de la reddition des comptes

- amélioration de la gestion des procédures par les gouvernements

- amélioration de la collecte des données

- amélioration de l'efficacité des contrôles

Source : adapté de (EBRD, 2015[3]).

Ces nombreux avantages ont un impact direct sur la gouvernance du système de passation des marchés publics, son efficience et, plus largement, sur le développement économique du pays. En termes de gouvernance, les procédures dématérialisées permettent un meilleur contrôle et examen de la gestion des dépenses publiques par différentes parties prenantes, y compris la société civile. Ceci permet d'améliorer la reddition des comptes, la gestion des dépenses publiques et donc la confiance des citoyens. En termes d'efficience, le e-procurement permet l'amélioration de la concurrence, la standardisation et la rationalisation des procédures, ce qui diminue les coûts administratifs et les délais pour les entités publiques comme pour les opérateurs économiques, permettant ainsi d'obtenir une diminution importante des prix. Dans certains cas, la mise en place du e-procurement permet d'atteindre des économies de l'ordre de 5 à $20 \%$ des dépenses en marchés publics (EBRD, 2015 $[3]$ ).Par ailleurs, en termes de développement économique, le e-procurement permet d'améliorer l'accès aux opportunités d'achat offertes par le secteur public aux fournisseurs potentiels quelque ce soit leur position géographique, ce qui améliore le développement des entreprises en particulier les PME.

Ces avantages ont incité de nombreux pays à initier depuis plusieurs années la mise en place de systèmes d'e-procurement. Le graphique 3.1 montre comment les systèmes électroniques de passation de marchés publics ont évolué dans le temps. 


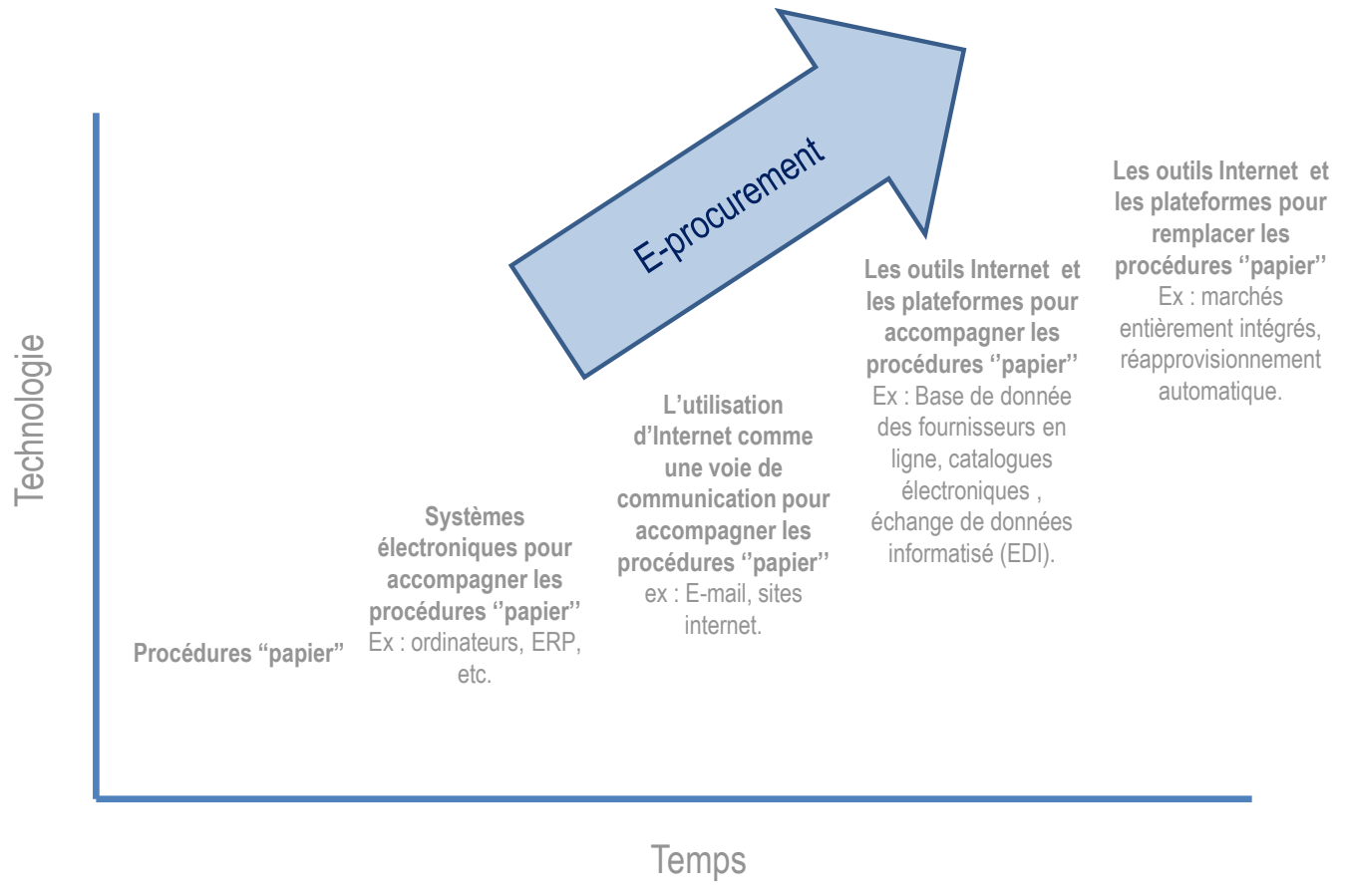

Source : (OCDE, 2018[13])

\subsubsection{Développer une stratégie globale pour la mise en place d'un système électronique de passation des marchés publics}

La mise en place d'un système de passation électronique des marchés publics représente un changement majeur pour les entités publiques et le secteur privé. Elle requiert le développement d'une stratégie adaptée afin d'assurer sa mise en place effective. Ces stratégies propres aux marchés publics font parfois partie d'une initiative plus large du gouvernement visant à mettre en place un gouvernement numérique, comme c'était le cas au Portugal et en Turquie, mais peuvent également être menées séparément (EBRD, $\left.2015_{[3]}\right)$. Comme le souligne la Recommandation de l'OCDE sur les stratégies numériques gouvernementales, le défi principal n'est pas d'introduire des technologies numériques dans l'administration publique, mais plutôt de s'assurer de l'intégration de leur utilisation dans les efforts de modernisation du secteur public (OCDE, 2014[14]).

En Algérie, le ministère de la Poste, des Télécommunications, des Technologies et du Numérique (MPTTN) est responsable de la politique de gouvernement électronique. À cet effet, il est chargé d'élaborer et de mettre en place plusieurs stratégies et projets visant à moderniser l'administration publique (Ministère de la Poste, des Télécommunications, des Technologies et du Numérique, s.d.[15]).

La mise en place d'une stratégie visant à mettre en place un système électronique de passation des marchés publics ne touche pas uniquement aux aspects techniques et technologiques de la plateforme, mais également à d'autres aspects (voir graphique 3.2) :

- Les aspects réglementaires : mise à jour du cadre réglementaire et des politiques afin d'assurer l'alignement avec les processus électroniques prévus dans le système (OCDE, 2018[13]).

- Le leadership institutionnel : ces systèmes nécessitant d'interagir avec plusieurs parties prenantes, il est nécessaire qu'une entité ait le leadership institutionnel. Un comité de pilotage où sont représentés toutes les parties prenantes, y compris le secteur privé et la société civile, comme cela a été fait au Mexique, est également nécessaire (OCDE, 2018[13]). 
- Les infrastructures informatiques et de télécommunication: le succès d'une stratégie d'e-procurement dépend du degré d'accès des acheteurs publics, mais aussi des entreprises, au système. II faut donc prendre en compte le taux et la qualité de la couverture internet et des équipements informatiques dans le pays concerné (EBRD, 2015[3]).

- La capacité du secteur privés et publics : les acheteurs publics et les fournisseurs potentiels ont besoin d'être sensibilisés et formés à l'utilisation d'un système électronique de passation des marchés publics. L'introduction d'une plateforme de dématérialisation permet de revoir les procédures en assurant une simplification administrative et une meilleure productivité. Cependant, cela implique également un changement des méthodes de travail que les autorités compétentes doivent prendre en compte (Commission Européenne, 2016[16]).

- Mobilisation du secteur privé : les marchés publics sont le domaine où se rencontrent le secteur public et le secteur privé. Pour la mise en place d'une telle stratégie, il est donc nécessaire de discuter et mobiliser le secteur privé, y compris les chambres de commerce et les associations d'entreprises et patronales, afin de les préparer à utiliser le système (OCDE, 2018[13]) .

- Les fonctionnalités du système doivent couvrir toutes les phases du cycle de passation des marchés publics et être conformes aux procédures décrites dans le cadre réglementaire. En termes de modèle économique, certains pays ont opté pour une plateforme électronique unique, alors que d'autres ont fait le choix d'ouvrir ce secteur à la concurrence avec un système de certification et de standardisation des procédures. Les questions liées au coût de fonctionnement doivent également être prises en compte afin de déterminer qui doit supporter le coût d'utilisation des services et des fonctionnalités offertes par la plateforme : l'État (par des subventions), les entités publiques ou les opérateurs économiques (EBRD, 2015[3]).

\section{Graphique 3.2. Les éléments nécessaires à la mise en place d'une stratégie de passation électronique des marchés publics}

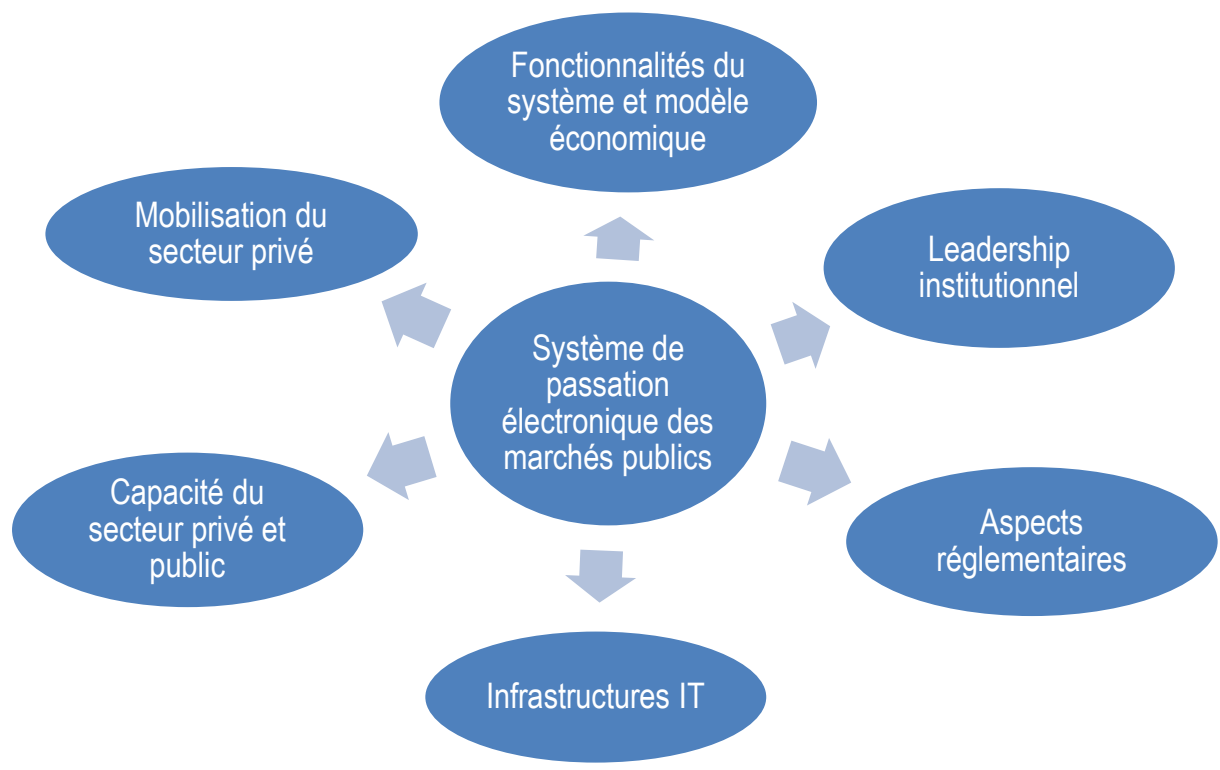

Note : IT: information et télécommunication.

Source : adapté de (EBRD, 2015[3]). 
Par ailleurs, le développement et la mise en place d'une stratégie d'e-procurement requièrent une approche méthodologique basée sur quatre étapes principales: i) l'analyse de l'existant; ii) le développement d'une stratégie ; iii) la préparation pour sa mise en place effective ; et iv) le réexamen de la situation (Commission Européenne, 2016[16]).

En Algérie, le ministre des Finances a publié un arrêté en novembre 2013 fixant le contenu du portail électronique des marchés publics, les modalités de sa gestion, ainsi que les modalités d'échange. Par ailleurs, le DMP, indique clairement que le MPTTN et le ministère des Finances sont conjointement en charge de gérer un portail électronique des marchés publics. Cependant, le contenu et les modalités de gestion du portail sont fixés par arrêté du ministre des Finances. Néanmoins, aucune stratégie spécifique n'a été développée, ce qui entrave la mise en place effective d'un portail électronique de passation des marchés publics. L'Algérie a donc reformé son cadre réglementaire et entamé le projet d'acquisition d'une plateforme électronique, sans pour autant prendre en compte les autres aspects qui sont nécessaires à la réussite de l'implémentation d'un système électronique de passation électronique des marchés publics.

D'après les missions exploratoires, le gouvernement algérien fait preuve d'une certaine volonté de moderniser l'administration publique, ce qui explique les fonds accordés au MPTTN pour mettre en place divers projets, dont la plateforme de dématérialisation des marchés publics. Le cadre réglementaire pour la mise en place d'une plateforme de dématérialisation est déjà publié depuis 2013 et 2015 mais aurait besoin d'être actualisé pour être conforme aux bonnes pratiques internationales et couvrir tout le cycle de passation des marchés publics. En termes d'équipements informatiques et de télécommunication, les autorités publiques ont assuré que toutes les administrations algériennes sont dotées d'ordinateurs et d'un accès internet. Cependant, la mission exploratoire relève que, dans certains cas, la qualité des équipements et du réseau internet reste à améliorer et le taux d'utilisation d'internet par le secteur privé reste limité. Par ailleurs, la capacité des agents en charge des marchés publics est l'une des lacunes centrales du système de passation des marchés publics (voir chapitre 6), il faudra donc que la stratégie de renforcement des capacités prenne en compte les aspects de dématérialisation. L'Algérie gagnerait à développer une stratégie pour la mise en place d'un système de passation électronique des marchés publics en suivant une approche méthodologique détaillée qui prend en compte l'analyse de l'existant et des besoins pour les aspects réglementaires, d'infrastructures informatiques et de télécommunication, de capacité des parties prenantes, de fonctionnalités et de modèle économique, de leadership institutionnel et de mobilisation du secteur privé.

Aucun comité de pilotage n'a été mis en place pour ce projet d'envergure. Les échanges entre le ministère des Finances et le MPTTN ne portent que sur les questions relatives au cahier des charges de la dématérialisation des marchés publics, qui n'est qu'un des aspects de la mise en place d'un système de passation électronique des marchés publics. Le développement de cette stratégie ne peut se faire sans la mise en place d'un comité de pilotage qui comprend le ministère des Finances, le MPTTN, les corps de contrôle, des représentants des entités publiques, les organismes de formations, les représentants du secteur privé et de la société civile.

Par ailleurs, les représentants du secteur privé ainsi que les entités publiques algériennes interrogés lors de la mission exploratoire ont indiqué ne pas être informés de la mise en place d'un système électronique de passation des marchés publics. Cette absence de communication peut nuire à la mise en place effective d'un tel système. En effet, en amont du renforcement des capacités des différentes parties prenantes sur ce sujet, ces dernières devraient être informées des réformes majeures menées dans ce domaine et pouvoir accéder à un espace où elles pourraient discuter de la mise en place du système et des éventuelles problématiques qui pourraient survenir lorsque l'utilisation du système sera obligatoire. C'est pour cela que de nombreux pays de l'OCDE ont mis en place de tels espaces, par exemple la Finlande, la France, l'Italie, la Norvège et la Suède (Commission Européenne, 2016 $\left.{ }_{[16]}\right]$.L'Algérie devrait proposer dès à présent un espace de discussion dédié à la mise en place du système de passation électronique des marchés publics et organiser des sessions d'information et de sensibilisation des parties prenantes afin de les préparer à la mise en place du système. De plus, si l'introduction de portails électroniques 
facilite l'accès des fournisseurs aux marchés publics sur le long terme, il pose également le défi de la formation des opérateurs économiques, et en particulier des PME, à l'utilisation de ces outils numériques. En 2016, $43 \%$ des pays de l'OCDE ont indiqué que le principal obstacle à l'utilisation des portails électroniques de marchés publics par les opérateurs économiques était des compétences insuffisantes en matière de technologie de l'information. II s'agit de l'obstacle le plus fréquemment cité, avant même la difficulté de comprendre les procédures ou les difficultés causées par des logiciels ou interfaces propriétaires (OCDE, 2016[17]).

D'autres pays qui ont réalisé la transition de modes de passation traditionnels des marchés publics vers les portails électroniques ont fait d'importants efforts de formation à destination des fournisseurs. Par exemple, la centrale d'achat public colombienne Colombia Compra Eficiente propose des manuels, des vidéos et des exercices en ligne afin d'expliquer le fonctionnement du portail de passation des marchés publics SECOP II. Un support technique/service d'assistance est à la disposition des utilisateurs du portail. Fort d'une trentaine d'employés qualifiés, il a traité 24533 requêtes en 2017, à la fois par téléphone, par courrier électronique et par messagerie en ligne (OCDE, 2018 $[13]$ ).

Au Mexique, entre 2012 et 2017, 14000 fournisseurs ont bénéficié d'une formation sur l'utilisation du portail électronique de passation des marchés publics CompraNet. Ce chiffre demeure faible par rapport au nombre de fournisseurs inscrits sur le portail en 2017 (environ 215000 fournisseurs) (OCDE, 2018[13]).

Enfin, le développement d'une stratégie de passation électronique des marchés publics doit également aborder les problématiques d'implémentation. En effet l'OCDE recommande aux pays d'adopter une approche par phases pour l'obligation de recours au système de passation électronique des marchés publics. II s'agit d'une approche commune dans les grands projets ou projets " transformateurs " car elle permet un meilleur suivi et contrôle de l'implémentation, d'une part, et elle réduit " l'impact du changement » pour les utilisateurs et parties prenantes au système, d'autre part. De plus, elle permet un ajustement de l'approche en réponse aux évolutions politiques et technologique (OCDE, 2018 $[13])$. Cette approche par phase doit prendre en compte plusieurs paramètres à décider par les autorités compétentes, notamment les fonctionnalités obligatoires du système et les catégories d'entreprises et d'entités publiques soumises à ces obligations, en imposant des dates précises d'entrée en vigueur de l'obligation d'utilisation des fonctionnalités pour chacune des catégories d'entités publiques.

Plusieurs pays ont adopté une telle approche progressive. En Tunisie par exemple, le système d'e-procurement Tuneps a été développé et mis en place en 2010, cependant ce n'est qu'en septembre 2018 que son utilisation est devenue obligatoire pour les ministères, entreprises publiques à caractère non administratif et autres établissements publics, alors que le ralliement des entreprises publiques et des collectivités locales est prévu en septembre 2019 (OCDE, 2019 [18]). En Algérie, la problématique de l'implémentation et du recours obligatoire au système de passation électronique des marchés publics n'a pas encore été définie. Ceci est important pour la sensibilisation et l'information des parties prenantes au système. Le gouvernement algérien devrait donc décider d'une approche progressive d'implémentation du système de passation électronique des marchés publics indiquant des dates précises d'obligation d'utilisation du système électronique par fonctionnalités et typologies d'entreprise le cas échéant.

\subsubsection{Pour l'implémentation d'un système électronique intégré aux autres systèmes de l'administration et couvrant toutes les phases de passation des marchés publics}

\section{Mettre en place un système de passation des marchés électroniques couvrant toutes les phases de passation des marchés publics}

Le système électronique de passation des marchés publics doit intégrer des technologies numériques récentes qui permettent d'appliquer des solutions intégrées couvrant tout le cycle de passation de ces marchés. Par ailleurs ce système devrait être modulaire, flexible, adaptable à différentes échelles et sûr 
afin d'assurer la continuité, la confidentialité et l'intégrité de l'activité, de garantir un traitement équitable, et de protéger les données sensibles. Les outils de la passation électronique de marchés publics devraient être faciles à utiliser, adaptés à leur objet et cohérents d'une entité publique à l'autre, dans la mesure du possible ; des systèmes trop compliqués pourraient en effet engendrer des risques et des difficultés de mise en œuvre pour les nouveaux entrants ou les PME (OCDE, 2015[1] $)$.

Conscients de la nécessité de couvrir toutes les phases de passation des marchés publics, la majorité des pays de l'OCDE ont mis en place des systèmes électroniques des marchés publics avec des fonctionnalités couvrant les phases de préparation des procédures (plans d'achat, avis de pré-information, avis et documents d'appels d'offres, de soumission [soumission électronique et enchères électroniques], etc.) et de gestion contractuelle (catalogues électronique, commandes en ligne et communication avec les fournisseurs, etc.) allant jusqu'à la fin du contrat (voir graphique 3.3).

\section{Graphique 3.3. Fonctionnalités des systèmes de passation de marchés électroniques dans les pays de l'OCDE}

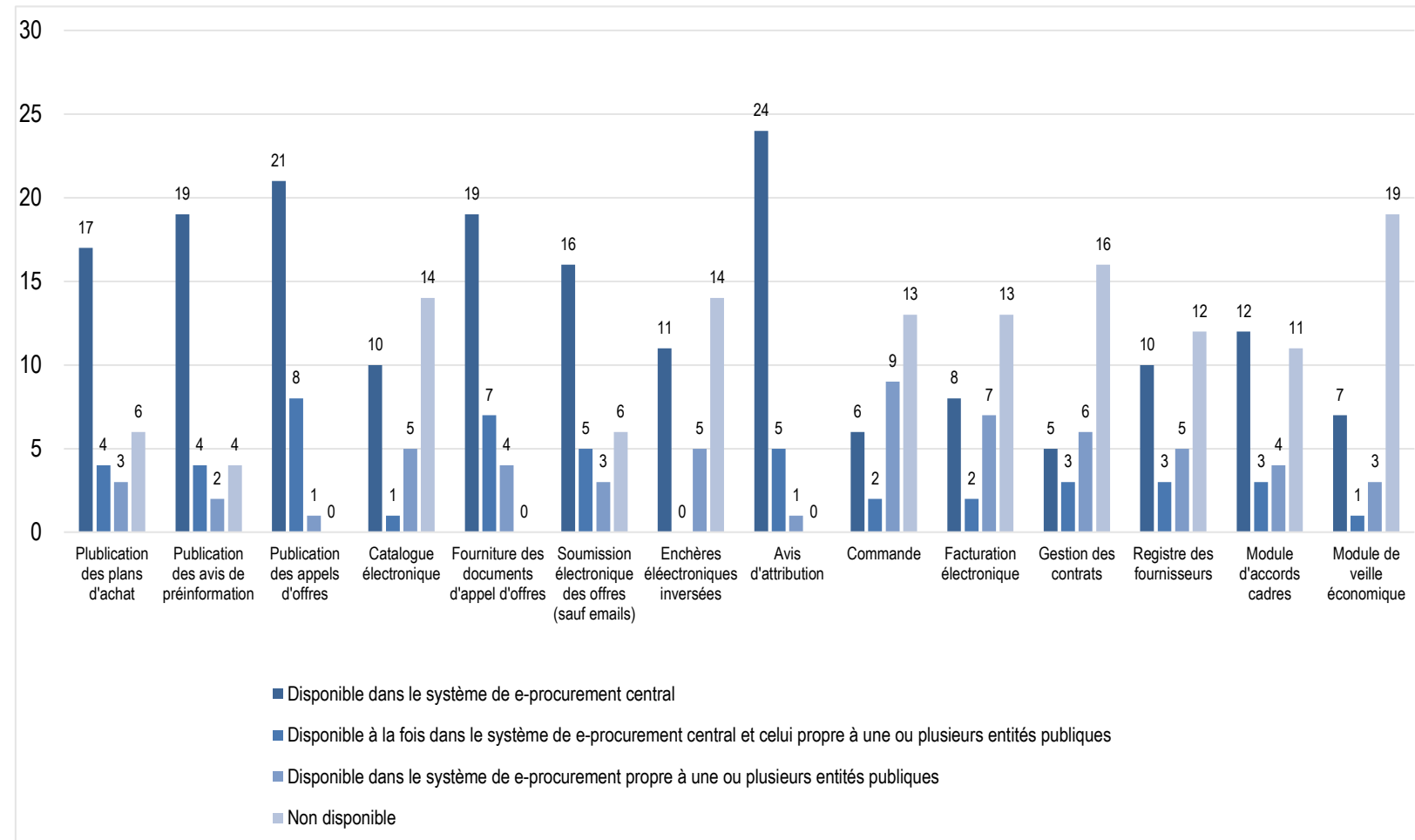

Source : (OCDE, 2017[19]).

En Algérie, le DMP prévoit la mise en place d'une unique plateforme gérée conjointement par le MPTTN et le ministère des Finances. Comme mentionné précédemment, dès 2013 le ministre des Finances a publié un arrêté qui fixe le contenu du portail électronique des marchés publics, les modalités de sa gestion ainsi que les modalités d'échange. Cependant, ce système n'a toujours pas été mis en place cinq ans après le premier arrêté du ministre des Finances et trois ans après la publication du DMP. L'encadré 3.5 décrit les fonctionnalités prévues dans le cadre réglementaire algérien. 
Encadré 3.5. Fonctionnalités principales du portail électronique des marchés publics prévues dans le cadre réglementaire algérien

- la publication des appels d'offres

- la communication des dossiers d'appel d'offres

- la soumission électronique des offres

- les enchères électroniques inversées

- la notification de l'attribution des marchés

- les catalogues électroniques

- la gestion des échanges d'informations entre les services contractants et les opérateurs économiques.

Source : DMP et arrêté du ministère des Finances.

Bien que ces fonctionnalités correspondent aux fonctionnalités principales attendues d'un système d'e-procurement, certaines fonctionnalités, telles que la transmission électronique des factures, ne sont pas clairement mentionnées. Le DMP prévoit cependant que « toute opération spécifique aux procédures sur support papier peut faire l'objet d'une adaptation aux procédures par voie électronique ».

En août 2018, l'équipe de l'OCDE a reçu un projet de cahier des charges relatif à la dématérialisation des marchés publics. Elle a émis des commentaires, relatifs entre autre à la nécessité de couvrir toutes les phases de passation des marchés publics, aux aspects liés à la transparence, à la nécessité de couvrir toutes les typologies de procédures sur la plateforme (pas uniquement les appels d'offres ouverts), ou encore à la nécessité d'introduire un système de classification des biens, produits et travaux publics. Ces éléments sont essentiels pour assurer un retour statistique complet sur les marchés publics, l'amélioration de la reddition des comptes et de l'efficience du système. Les autorités compétentes ont assuré que ces commentaires seraient pris en compte dans la version définitive du cahier des charges relatif à la dématérialisation des marchés publics.

L'Algérie a pris du retard dans la mise en place d'un système électronique de passation des marchés publics. Le ministère des Finances et le MPTTN devraient veiller à mettre ce système en place le plus rapidement possible, en s'assurant que ses fonctionnalités couvrent toutes les phases de passation des marchés publics.

\section{S’assurer de la possibilité d'obtenir les documents nécessaires à la soumission électronique des offres sous format dématérialisé}

La soumission électronique des offres est une fonctionnalité clé dans le système de passation électronique des marchés publics. Lors de cette étape, les soumissionnaires doivent envoyer des documents sous format électronique aux acheteurs publics afin de leur permettre d'apprécier leurs capacités techniques, professionnelles et financières, mais aussi d'évaluer la qualité de leurs offres. Certains de ces documents ont une valeur juridique, il est donc nécessaire qu'ils soient disponibles sous format électronique. (EBRD, 2015[3])

Le modèle de dématérialisation choisi par l'Algérie inclut la signature électronique. Cependant, certains des documents fournis par l'administration publique, les banques ou d'autres organismes doivent également être fournis sous format électronique. Ceci nécessite donc des discussions préalables avec les différentes autorités ou organismes afin qu'elles puissent être préparées à délivrer ces documents sous 
format électronique. En Algérie, ces documents incluent les statuts pour les sociétés, les certificats de qualification et de classification (ou agrément/certificat de qualité), les bilans et les références bancaires, ainsi que les cautions bancaires. Lors des missions exploratoires, les autorités responsables ont mentionné ne pas encore avoir abordé cette problématique. Le gouvernement algérien devrait donc prendre les mesures nécessaires pour assurer la délivrance de tous les documents nécessaires lors de la soumission électronique sous format dématérialisé.

\section{Intégrer le système de passation des marchés électroniques aux autres systèmes de l'administration}

Le domaine des marchés publics a énormément évolué grâce aux avancées technologiques. II nécessite une intégration verticale et horizontale avec plusieurs systèmes d'informations (voir graphique 3.4). En ce qui concerne l'intégration verticale, l'OCDE recommande de rationaliser les dépenses liées à la passation de marchés publics en intégrant les processus de passation des marchés dans le cadre général de la gestion des finances publiques, ce qui comprend les systèmes budgétaires, fiscaux et de sécurité sociale. En effet, ceci permet d'améliorer l'efficience du système et diminuer la charge administrative des entreprises et des entités publiques (OCDE, 2015 [1] $)$.L'amélioration de l'efficacité du système nécessite également une intégration verticale avec les systèmes d'information et progiciels de gestion intégrée des entités publiques, en particuliers ceux relatifs à la gestion contractuelle et au paiement (OCDE, 2018[13]). L'encadré 3.6 décrit le travail effectué en Finlande pour intégrer le système de passation des marchés électroniques à d'autres systèmes de l'administration.

\section{Graphique 3.4. Intégration verticale et horizontale du système de passation électronique des marchés publics}

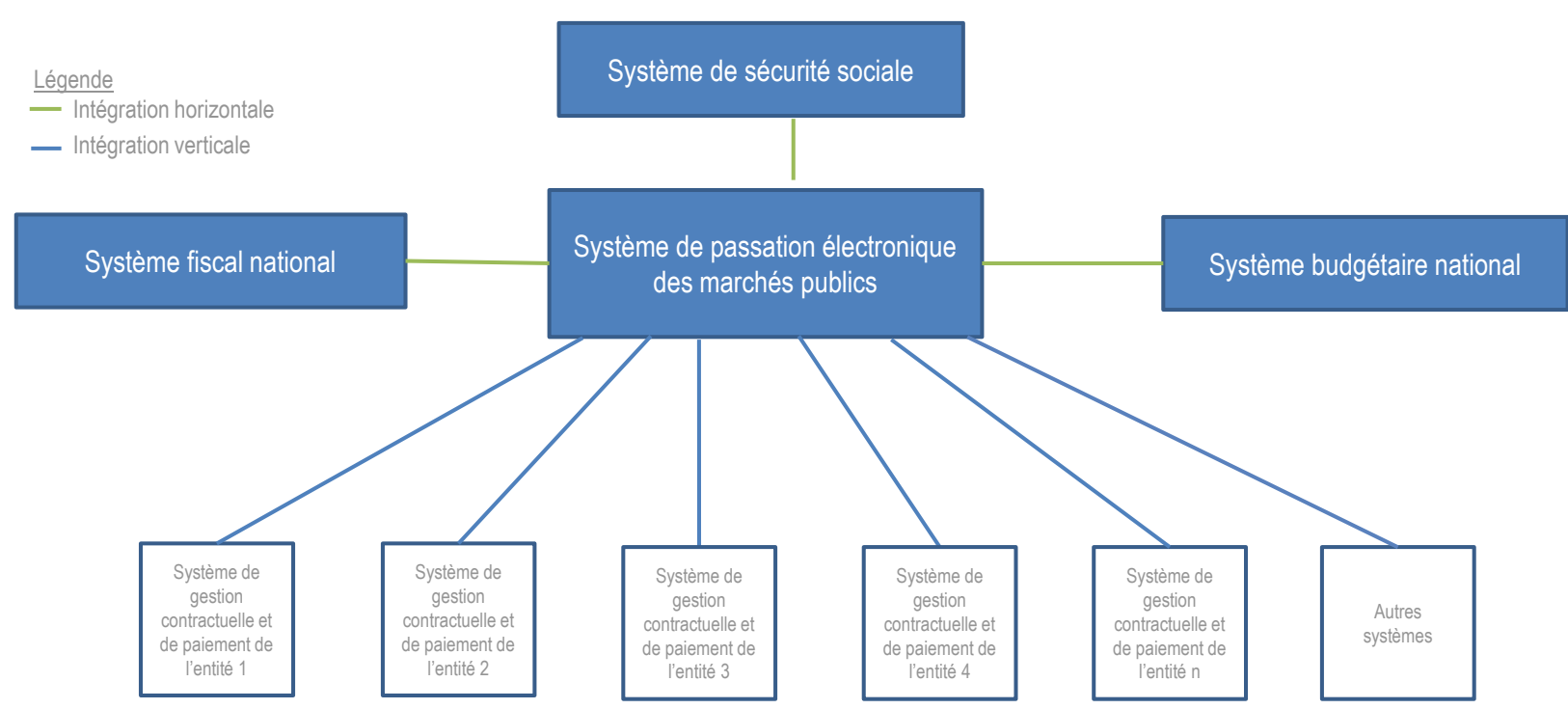

Source : adapté de (OCDE, 2018[13]). 


\section{Encadré 3.6. Intégration du système de passation des marchés électroniques à d'autres systèmes de l'administration en Finlande}

Le Document unique de marché européen (DUME) est un formulaire par lequel les entreprises déclarent leur statut financier, leurs capacités et leur aptitude à participer à une procédure de marché public. Il est disponible dans toutes les langues de l'UE et prouve a priori que les conditions requises pour participer aux procédures de passation de marchés publics dans l'UE sont remplies. Grâce au DUME, les soumissionnaires ne doivent plus fournir ni les justificatifs ni les différents formulaires utilisés auparavant dans le cadre de procédures de passation de marchés publics, ce qui signifie que l'accès aux offres publiées dans d'autres États est sensiblement simplifié.

En Finlande, le DUME est intégré au système de passation électronique Hanki utilisé principalement par les entités du gouvernement central finlandais. II permet donc d'exclure automatiquement les opérateurs économiques qui ne remplissent pas les critères de sélection et ceux qui remplissent les critères d'exclusion fixés par l'acheteur.

Afin de faciliter le processus, la Finlande a intégré automatiquement le DUME aux bases de données nationales suivantes:

- registre du commerce et registre des hypothèques d'entreprise

- registre des employeurs, registre des paiements anticipés, registre de la TVA, actionnaires, etc.

- registre des assurances et pensions de retraite

- registre de l'assurance chômage

- registre de l'assurance contre les accidents du travail

- registre des douanes

- registre des entreprises en faillite et en restructuration

- registre des casiers judiciaires.

Les données sont donc extraites des bases de données pour être réutilisées à des fins de passation des marchés, ce qui réduit le nombre d'attestations et de documents que les opérateurs économiques doivent fournir lors de la procédure.

Source : (Commission Européenne, s.d.[20]).

En Algérie, d'après la mission exploratoire, les problématiques d'intégration du système de passation électronique des marchés publics avec le système national fiscal et budgétaire n'ont pas été abordées. Le gouvernement algérien gagnerait donc à prévoir dès à présent l'intégration du système de passation électronique des marchés publics avec d'autres systèmes d'information de l'administration publique afin d'améliorer l'efficience du système et diminuer la charge administrative des entreprises et des entités publiques.

\section{Un système de passation électronique des marchés publics qui permet de collecter des données et de les publier}

La dématérialisation du système de passation des marchés publics permet d'améliorer l'efficience et la productivité du système, mais aussi la confiance des citoyens. En effet, il est du devoir des gouvernements de fournir à la société civile, ainsi qu'à toute partie prenante des informations claires sur la manière dont l'argent des contribuables est dépensé (OCDE, 2019[21]). Afin de tirer profit du système de passation électronique des marchés publics, ce dernier ne doit pas uniquement stocker les informations, mais 
également les collecter de façon à produire des données structurées, ouvertes, partageables et réutilisables. Ainsi, lorsque les pays développent des systèmes électroniques de passation des marchés publics, ils doivent également mettre en place des standards adéquats en prenant en compte les rapports et informations à partager ainsi que les données nécessaires pour le faire (OCDE, 2018[22]). Plusieurs initiatives visent à promouvoir ce type de standards, comme par exemple l'initiative des Standard de données sur la commande publique ouverte(Open Contracting Data Standards) (Open Contracting Partnership, s.d.[23]).

En Algérie, les questions relatives à la collecte des données et à leur utilisation n'ont pas encore été abordées en raison de l'absence d'indicateurs sur les marchés publics (voir chapitre 3). Le gouvernement algérien gagnerait à mettre en place un système de dématérialisation des marchés publics basé sur des données structurées et réutilisables pour améliorer la transparence du système et son efficacité.

\section{Propositions d'action}

\begin{tabular}{|c|c|c|c|}
\hline Recommandations & $\begin{array}{l}\text { Court } \\
\text { terme }\end{array}$ & $\begin{array}{l}\text { Moyen } \\
\text { terme }\end{array}$ & $\begin{array}{l}\text { Long } \\
\text { terme }\end{array}$ \\
\hline Publier des avis de pré-information & $\mathrm{X}$ & & \\
\hline Publier des informations relatives à la gestion contractuelle & & & $\mathrm{X}$ \\
\hline Publier les rapports des corps de contrôle a posteriori & & & $\mathrm{X}$ \\
\hline $\begin{array}{l}\text { S'assurer du respect des obligations de transparence concernant les programmes prévisionnels et la liste } \\
\text { des opérateurs économiques exclus des marchés publics }\end{array}$ & & $x$ & \\
\hline $\begin{array}{l}\text { Rendre gratuit et instantané l'accès aux informations publiées sur le Bomop et améliorer l'organisation } \\
\text { des différentes rubriques du Bomop }\end{array}$ & & $X$ & \\
\hline $\begin{array}{l}\text { Réfléchir au maintien ou non la publication des annonces aux Bomop, lors de la mise en place du } \\
\text { e-procurement }\end{array}$ & & & $\mathrm{X}$ \\
\hline $\begin{array}{l}\text { Doter toutes les entités publiques d'un site web et y publier les informations relatives à leurs marchés } \\
\text { publics }\end{array}$ & & & $\mathrm{X}$ \\
\hline $\begin{array}{l}\text { Mettre en ligne une présentation hiérarchisée et exhaustive du cadre règlementaire des marchés publics } \\
\text { (DMP et arrêtés d'application) sur une page web spécifique }\end{array}$ & $X$ & & \\
\hline Améliorer la qualité du site web du journal officiel & $X$ & & \\
\hline Collecter et diffuser des données sur la performance des marchés publics & & & $\mathrm{X}$ \\
\hline $\begin{array}{l}\text { Mettre en place une politique permettant aux acheteurs publics de pouvoir apprécier la confidentialité de } \\
\text { l'information à publier }\end{array}$ & & $\mathrm{X}$ & \\
\hline $\begin{array}{l}\text { Développer une stratégie pour la mise en place d'un système de passation électronique des marchés } \\
\text { publics }\end{array}$ & $\mathrm{X}$ & & \\
\hline $\begin{array}{l}\text { Mettre en place un comité de pilotage pour le développement de la stratégie pour la mise en place du } \\
\text { système de passation électronique des marchés publics }\end{array}$ & $X$ & & \\
\hline $\begin{array}{l}\text { Mettre en place un espace de discussion dédié à la mise en place du système de passation électronique } \\
\text { des marchés publics }\end{array}$ & $X$ & & \\
\hline $\begin{array}{l}\text { Organiser des sessions d'information et de sensibilisation des différentes parties prenantes afin de les } \\
\text { préparer à la mise en place du système de passation électronique des marchés publics }\end{array}$ & $\mathrm{X}$ & & \\
\hline $\begin{array}{l}\text { Prévoir des formations adaptées à destination des opérateurs économiques du secteur privé sur son } \\
\text { système de passation électronique des marchés publics }\end{array}$ & & $X$ & \\
\hline $\begin{array}{l}\text { Décider d'une approche progressive d'implémentation du système de passation électronique des } \\
\text { marchés publics }\end{array}$ & $\mathrm{X}$ & & \\
\hline $\begin{array}{l}\text { Mettre en place le système de passation électronique des marchés publics tout en s'assurant que ses } \\
\text { fonctionnalités couvrent toutes les phases de passation des marchés publics. }\end{array}$ & & $x$ & \\
\hline $\begin{array}{l}\text { Prendre les mesures nécessaires pour assurer la délivrance de tous les documents nécessaires lors de } \\
\text { la soumission électronique sous format dématérialisé }\end{array}$ & & $x$ & \\
\hline $\begin{array}{l}\text { Prévoir l'intégration du système de passation électronique des marchés publics avec les autres systèmes } \\
\text { d'information de l'administration }\end{array}$ & & & $\mathrm{X}$ \\
\hline $\begin{array}{l}\text { Mettre en place un système de dématérialisation des marchés publics basé sur des données structurées } \\
\text { et réutilisables }\end{array}$ & & $\mathrm{X}$ & \\
\hline
\end{tabular}

Note : Court terme : recommandation à mettre en application dans les 12 mois suivants ;

Moyen terme : recommandation à mettre en application dans les $1-2$ ans ;

Long terme : recommandation à mettre en application dans plus de 2 ans. 


\section{Références}

Commission Européenne (2016), Governance and Capacity Building- EXEP subgroup report, http://ec.europa.eu/DocsRoom/documents/20844 (consulté le 13 décembre 2018).

Commission Européenne (s.d.), Le document unique de marché européen, 2018, https://ec.europa.eu/tools/espd/filter?lang=fr (consulté le 22 février 2019).

Crown Commercial Service (2017), General Legal Advice Services, https://ccsagreements.cabinetoffice.gov.uk/contracts/rm3786 (consulté le 18 juillet 2018).

Direction de l'information légale et administrative (DILA), France (s.d.), Bulletin officiel des annonces des marchés publics (BOAMP), https://www.boamp.frl (consulté le 17 décembre 2018).

EBRD (2015), Are you ready for eProcurement? Guide to Electronic Procurement Reform, https://www.ppi-ebrduncitral.com/images/stories/pdf/151208 Are you ready for eProcurement.pdf (consulté le 13 décembre 2018).

Ministère de la Poste, des Télécommunications, des Technologies et du Numérique (s.d.), EGov, https://www.mpttn.gov.dz/fr/content/administration-\%C3\%A9lectronique (consulté le 13 décembre 2018).

Ministère des Finances, Algérie (s.d.), Réglementation des marchés publics, http://www.mf.gov.dz/article/14/Autres-Textes/355/R\%C3\%A9glementation-desmarch\%C3\%A9s-publics.html (consulté le 14 décembre 2018).

OCDE (2019), Améliorer l'accès des PME aux marchés publics en Tunisie: la voie à suivre, OCDE, Paris, http://www.oecd.org/countries/tunisia/ameliorer-acces-PME-aux-marchespublics-Tunisie.pdf (consulté le 11 juin 2019).

OCDE (2019), Productivity in Public Procurement, A Case Study of Finland: Measuring the Efficiency and Effectiveness of Public Procurement, OCDE, Paris, https://www.oecd.org/gov/public-procurement/publications/productivity-publicprocurement.pdf (consulté le 31 juillet 2019).

OCDE (2018), Mexico's e-Procurement System: Redesigning CompraNet through Stakeholder Engagement, Examens de l'OCDE sur la gouvernance publique, Éditions OCDE, Paris, https://dx.doi.org/10.1787/9789264287426-en.

OCDE (2018), Second Public Procurement Review of the Mexican Institute of Social Security (IMSS) : Reshaping Strategies for Better Healthcare, Examens de l'OCDE sur la gouvernance publique, Éditions OCDE, Paris, https://dx.doi.org/10.1787/9789264190191-en.

OCDE (2017), OECD Integrity Review of Mexico: Taking a Stronger Stance Against Corruption, Examens de l'OCDE sur la gouvernance publique, Éditions OCDE, Paris, https://dx.doi.org/10.1787/9789264273207-en.

OCDE (2017), Panorama des administrations publiques 2017, Éditions OCDE, Paris, http://dx.doi.org/10.1787/gov glance-2017-fr. 
OCDE (2017), Recommandation du Conseil de l'OCDE sur l'intégrité publique, OCDE, Paris, http://www.oecd.org/gov/ethics/Recommandation-integrite-publique.pdf (consulté le 20 juillet 2018).

OCDE (2016), Checklist for Supporting the Implementation of OECD Recommendation of the Council on Public Procurement: Transparency, https://www.oecd.org/governance/procurement/toolbox/search/Checklist\%2001\%20Transpar ency.pdf (consulté le 14 décembre 2018).

OCDE (2016), « Réponses au questionnaire de l'OCDE sur les marchés publics ».

OCDE (2015), Recommandation du Conseil de l'OCDE sur les marchés publics, OCDE, Paris, http://www.oecd.org/fr/gov/ethique/Recommandation-OCDE-sur-les-marches-publics.pdf (consulté le 16 août 2018).

OCDE (2014), Recommendation of the Council on Digital Government Strategies, OCDE, Paris, http://www.oecd.org/gov/digital-government/Recommendation-digital-governmentstrategies.pdf (consulté le 13 décembre 2018).

OCDE (à paraître), Enhancing the Use of Competitive Tendering in Costa Rica's Public Procurement System, OCDE, Paris.

Open Contracting Partnership (s.d.), Open Contracting Data Standard: Documentation - OCDS 1.1.3 documentation, http://standard.open-contracting.org/latest/en/\# (consulté le 17 décembre 2018).

Organismo Supervisor de las Contrataciones del Estado (OSCE)- Perou (s.d.), Études et statistiques sur le système de passation des marchés publics, http://portal.osce.gob.pe/osce/content/estadisticas (consulté le 17 décembre 2018).

Secrétariat général du gouvernement, Algérie (s.d.), Journal Officiel.

Transparency International (2014), Public procurement- topic guide, http://www.transparency.org (consulté le 18 décembre 2018). 


\section{Optimiser les marchés publics en Algérie pour une plus grande efficience}

L'amélioration de l'efficience du système de passation des marchés publics est un enjeu majeur en Algérie au vu du volume financier en jeu. Ce chapitre analyse les forces et les opportunités d'amélioration de l'efficience du système du point de vue réglementaire, mais aussi dans la pratique des entités publiques. II fournit des recommandations au pays visant à améliorer le système en place tout au long du cycle de passation des marchés publics, et ce, dans différents domaines. Ce chapitre traite également du développement de l'évaluation du système de passation des marchés publics, encore absente en Algérie. Enfin, ce chapitre présente des recommandations destinées à combler ces lacunes. 
La nécessité de répondre efficacement aux besoins des citoyens en termes d'infrastructures et de services est l'un des objectifs principaux de la commande publique. Dans les pays de l'OCDE, les marchés publics représentent près de $12 \%$ du produit intérieur brut (PIB) et $29 \%$ de l'ensemble des dépenses publiques en 2015 (OCDE, 2017[1]). En Algérie, ils représentent plus de $20 \%$ du PIB. Étant donné les volumes financiers en jeu, les gouvernements doivent garantir une bonne gestion des fonds publics afin qu'ils apportent à leurs citoyens la meilleure valeur ajoutée possible (EBRD, 2015[2]). À cet égard, la Recommandation de l'OCDE sur les marchés publics met l'accent sur l'optimisation de l'efficience à tous les stades du cycle de passation des marchés publics. Par ailleurs, cette efficience ne peut être mesurée sans une évaluation régulière de la performance du système tant au niveau de chaque procédure que pour le système de passation des marchés publics dans son ensemble (OCDE, 2015[3]).

L'efficience dans les marchés publics nécessite une approche stratégique. En effet, les marchés publics ont évolué au cours des dernières années, passant d'une approche traditionnelle à une approche stratégique (voir graphique 4.1). Dans l'approche traditionnelle des marchés publics, les efforts sont concentrés sur la phase de passation des marchés, alors que l'approche stratégique met davantage l'accent sur la phase préparatoire (évaluation des besoins, conduite des études de marché et rédaction des cahiers des charges) et sur l'exécution du contrat. Afin d'améliorer l'efficience du système de passation des marchés publics, plusieurs pays ont adopté cette approche stratégique couplée avec l'utilisation d'outils d'efficience et de cahiers des charges adaptés à la réalité du marché. 


\section{Graphique 4.1. Approche traditionnelle et stratégique des marchés publics}

Approche traditionnelle des marchés publics

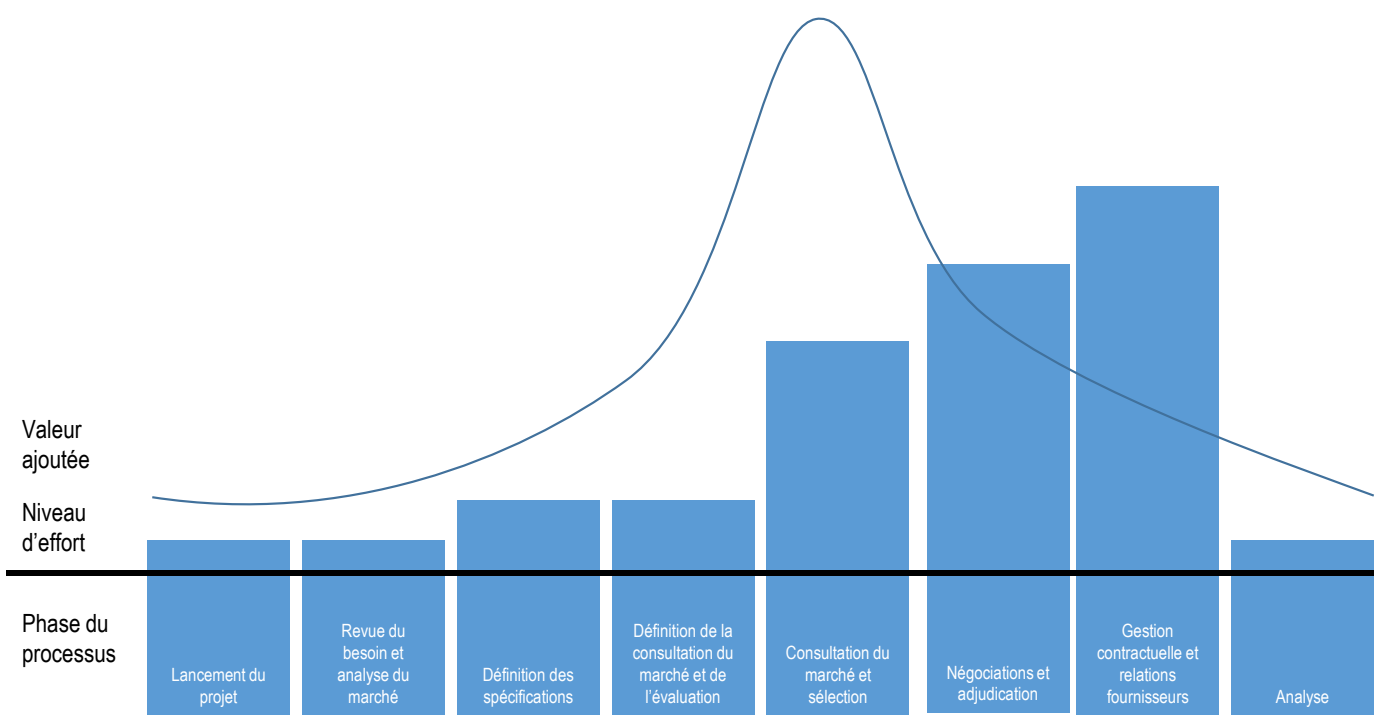

Approche stratégique des marchés publics

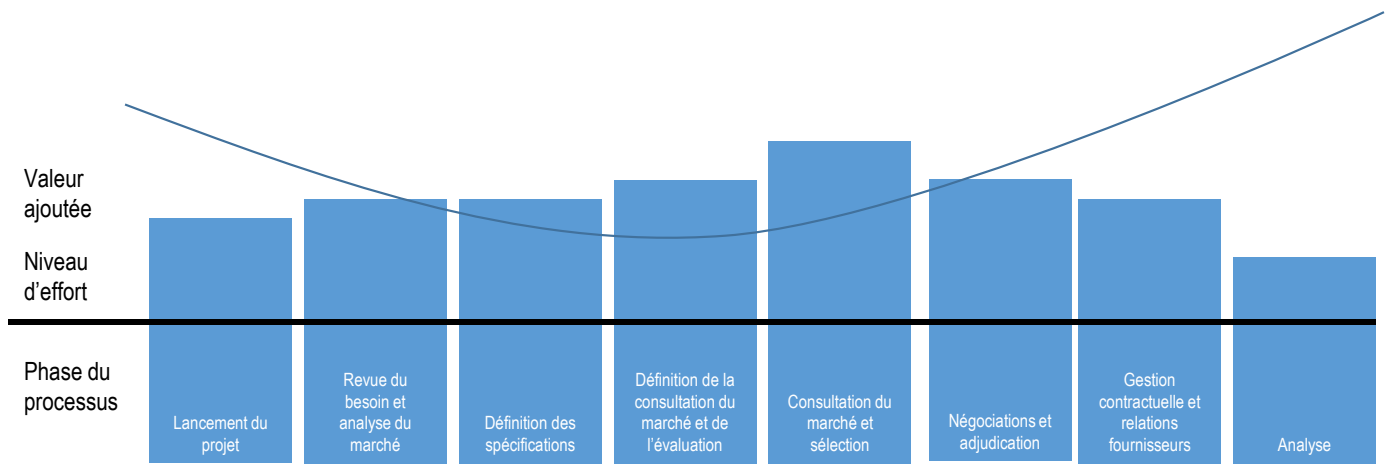

Source : (Ministry of Business, Innovation and Employment, 2011[4]).

En Algérie, les préoccupations d'efficience sont d'autant plus importantes compte tenu de l'environnement économique qu'a connu le pays ces dernières années (Banque Mondiale, $2018_{[5]}$ ). Le pays a connu en 2015 une réforme de son cadre réglementaire régissant son système de passation des marchés publics. Le décret présidentiel $n^{\circ} 15-247$ du 16 septembre 2015 portant réglementation des marchés publics et des délégations de service public (DMP) a introduit certains des outils visant à améliorer l'efficience du système de passation des marchés publics. Cependant des améliorations supplémentaires pourraient avoir un impact positif sur l'efficacité du système. Par ailleurs, aucune évaluation de l'efficacité du système de passation des marchés publics n'a encore été menée.

Ce chapitre analyse les outils dont dispose l'Algérie pour améliorer l'efficience du système de passation des marchés publics et fournit des recommandations conformes aux meilleures pratiques internationales. II traite en particulier de l'évaluation des besoins, des études de marché, du choix de critères de qualification et de sélection adaptés et des outils d'efficience. II traite également de l'évaluation du système 
de passation des marchés publics, qui est fondamentale pour améliorer l'efficience du système (OCDE, $\left.2015_{[3]}\right)$.

\subsection{L'évaluation des besoins et l'analyse des marchés, une étape clé pour la performance du système de passation des marchés publics}

L'efficacité des marchés publics repose tout d'abord sur l'adéquation des besoins des pouvoirs adjudicateurs avec la réalité du marché, et donc les offres des fournisseurs potentiels. L'étape de l'analyse des besoins et la réalisation d'études de marché est donc une étape cruciale, qui est parfois négligée si l'entité publique n'adopte pas une approche stratégique des marchés publics. Cette étape permet en effet aux entités publiques de comprendre dans quelle mesure le marché a la capacité de satisfaire leurs besoins, d'une part, et elle permet aux opérateurs économiques de mieux comprendre les besoins des entités publiques d'autre part (graphique 4.2). Ces analyses déterminent largement le choix de la procédure de passation du marché et la conception des documents d'appel d'offres, et auront donc un impact sur la qualité de l'exécution du marché (OCDE, 2018[6] ). C'est pourquoi les meilleures pratiques internationales considèrent l'analyse des besoins et les études de marché comme une activité stratégique, en non une simple tâche administrative.

\section{Graphique 4.2. L'impact de l'analyse des besoins et de l'analyse du marché sur l'appel d'offres}

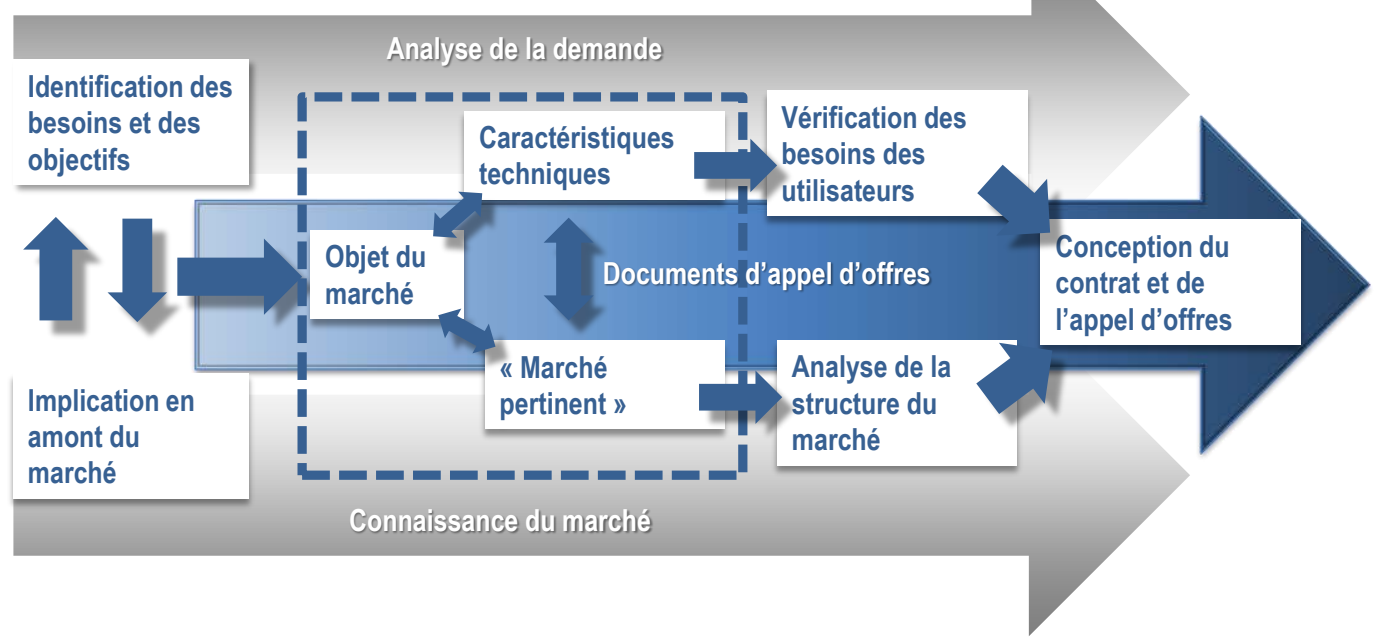

Source : (OCDE, 2018[6]).

\subsubsection{Développer et harmoniser l'analyse et la planification des besoins}

L'analyse du besoin est une étape essentielle du cycle de passation des marchés publics qui permet aux entités publiques de justifier toute procédure d'achat (Union Européenne, 2014[7]).L'entité publique identifie les éléments nécessaires à la mise en œuvre de ses missions de service public qu'elle ne peut obtenir en puisant dans ses ressources internes. L'analyse du besoin n'est donc pas limitée aux quantités de biens et services nécessaires, elle doit être centrée sur les fonctionnalités que doivent remplir les solutions attendues. 


\section{Des programmes prévisionnels standardisés basés sur une analyse fonctionnelle des} besoins

Les bonnes pratiques des pays de l'OCDE consistent à conduire une analyse fonctionnelle des besoins formulés, en termes de solutions et non de produits et services disponibles sur le marché. Une telle approche permet par la suite de ne pas limiter la définition du marché pertinent, et donc d'augmenter le niveau de concurrence (Mabin, 2018[8]).

En Algérie, l'article 27 du DMP traite de la détermination des besoins, il précise que la nature et l'étendue du besoin du service contractant doivent être définies « avec précision, par référence à des spécifications techniques détaillées établies sur la base de normes et/ou performances ou d'exigences fonctionnelles. Ces spécifications techniques ne doivent pas être orientées vers un produit ou un opérateur économique déterminé ». L'établissement des besoins par référence à des exigences fonctionnelles exige un certain niveau de capacité dans les entités publiques. Les missions exploratoires de l'OCDE suggèrent qu'en raison des capacités insuffisantes des services contractants, les acheteurs publics se réfèrent souvent aux normes et solutions utilisées lors des procédures d'achat antérieures. Dès lors, le renforcement des capacités des services contractants en matière d'analyse des besoins contribuerait à ancrer l'analyse fonctionnelle dans les pratiques des acheteurs publics en Algérie et à améliorer l'efficience du système.

Une fois le besoin identifié, il doit être intégré dans le plan d'achat de l'entité publique. La publication des plans d'achat en avance est nécessaire afin de donner de la visibilité aux opérateurs économiques sur les opportunités d'achat qui leur sont offertes. Dans la plupart des pays de l'OCDE, les autorités publient leurs plans d'achat sur leur système de passation électronique des marchés, il s'agit d'ailleurs souvent d'une exigence réglementaire (OCDE, 2016[9] $)$. Ainsi, par exemple, en Nouvelle-Zélande les entités publiques doivent publier les procédures dans leurs plans d'achats détaillés au moins un an avant leur lancement, et mettre à jour le plan d'achat au moins une fois tous les six mois (New Zealand Government Procurement Branch, 2015[10]). Par ailleurs, les bonnes pratiques internationales prévoient le développement d'un format standardisé de programmes prévisionnels d'achat, qui permettra d'harmoniser la présentation pour les opérateurs économiques et de s'assurer que les programmes prévisionnels fournissent un niveau de détail pertinent, suffisant et homogène (objet de la procédure, catégories de biens et services, quantités ou montants ainsi que date approximative de lancement de la procédure, etc.). Ces standards devraient également inciter les services contractants à formuler le besoin sous forme d'exigence fonctionnelle, de façon à susciter l'intérêt d'un plus grand nombre d'opérateurs économiques. (Gouvernment d'Australie, $\left.2019_{[11]}\right)$

D'après la réglementation algérienne, les services contractants sont tenus de publier un programme prévisionnel des projets de marché au début de chaque exercice budgétaire au Bulletin officiel des marchés de l'opérateur public (Bomop) et/ou sur leur site internet. Cependant, comme mentionné au chapitre 3, les programmes prévisionnels ne sont publiés ni sur le Bomop ni sur le site web des entités publiques. Ce manque de transparence peut avoir un impact négatif sur le niveau de participation des fournisseurs potentiels aux opportunités d'achat, et donc sur le niveau de concurrence. Par ailleurs, le cadre réglementaire ne prévoit pas de format spécifique pour les programmes prévisionnels, ils peuvent donc être préparés dans des formats différents par chaque entité publique. En plus des mesures pour s'assurer du respect des obligations de transparence relatives aux programmes prévisionnels, le gouvernement algérien devrait donc développer un format standard pour le programme prévisionnel des projets de marché.

\section{La participation des utilisateurs finaux à la définition des besoins}

Une définition adéquate des besoins nécessite de consulter les utilisateurs finaux des biens, services ou travaux publics et d'établir un dialogue avec eux. Étant donné que l'objectif des marchés publics est de fournir des services et infrastructures aux citoyens, mais aussi d'acquérir des biens et services et travaux publics permettant aux entités publiques de mener à bien leur mission de service public, il est possible de 
distinguer deux catégories d'utilisateurs: les services au sein de l'entité publique dont les missions nécessitent l'acquisition de biens et services ou de travaux publics, d'une part, et les citoyens d'autre part.

Les besoins des citoyens peuvent être collectés et décidés au niveau central ou local. Au niveau local, les élus des assemblées communales populaires consultent les populations afin d'identifier les besoins liés aux services publics locaux en allant à leur rencontre. Les assemblées populaires et les exécutifs locaux s'attellent ensuite à la priorisation des besoins et à l'élaboration du Plan communal de développement (PCD), où sont retracées l'ensemble des opérations d'investissement programmées par l'Assemblée populaire communale. Les PCD sont financés par une enveloppe budgétaire nationale qui est ensuite affectée à chaque commune à travers des séances d'arbitrages organisées au niveau des wilayas (Ministère de I'Intérieur, Algérie, 2015[12]). Au niveau de la wilaya, chaque département sectoriel (Santé, éducation, etc.) exprime ses besoins, l'assemblée populaire de la wilaya est également consultée. Le wali joue ensuite un rôle d'arbitrage, avant la soumission des propositions budgétaires au gouvernement. Après leur approbation, les projets d'investissement sont inscrits au Programme sectoriel déconcentré (PSD). Le wali et ses services sont responsables de son exécution (Banque Mondiale, 2007[13]). Au niveau central, le budget d'investissement des administrations centrales et établissement publics sous leur tutelle est approuvé par le gouvernement. Une fois inscrits à la nomenclature des dépenses d'investissements publics de l'État, ils s'articulent en programmes sectoriels centralisés (PSC). II s'agit de projets d'équipement d'envergure nationale, tels que la réalisation d'un barrage, d'une infrastructure de transport, ou l'acquisition d'équipements lourds. Ces projets sont gérés directement par les ministères sectoriels au niveau central (Banque Mondiale, 2007[13]).

En Algérie, pour les besoins locaux, il existe ainsi un processus de consultation des citoyens et donc des utilisateurs, ce qui est considéré comme une bonne pratique internationale. Cependant, ce processus n'est pas formalisé. Plusieurs pays ont mis en place des systèmes de consultation ou de participation citoyenne. Au niveau local, les budgets participatifs, par exemple, sont des outils de participation citoyenne qui peuvent être pertinents pour l'identification des besoins des citoyens (encadré 4.1). L'Algérie gagnerait à formaliser le processus de consultation citoyenne afin de s'assurer de façon plus systématique que les infrastructures et services offerts aux citoyens correspondent à leurs besoins.

Au niveau central et au niveau des Wilayas, l'Algérie gagnerait à mettre en place des études plus poussées et formalisées de manière à mieux identifier et prioriser les besoins en matière de dépenses d'équipement. Il s'agit notamment de renforcer les études d'identification et de faisabilité des investissements dans la phase amont de la maturation des projets par les ministères sectoriels. Ces études visent notamment à prioriser les projets et à écarter les " éléphants blancs » (projets inutiles et coûteux) (Caisse nationale d'équipement pour le développement (CNED), 2008[14]).

En outre, une fois des mécanismes formels de consultation des citoyens mis en place au niveau des communes, l'Algérie pourrait envisager de les utiliser également dans l'identification et la priorisation des besoins au niveau de chaque Wilaya, dans le cadre de l'élaboration des plans sectoriels déconcentrés. 


\section{Encadré 4.1. Les budgets participatifs}

Les budgets participatifs permettent aux citoyens de se prononcer sur une partie de l'allocation du budget d'une collectivité territoriale, généralement le budget d'investissement. Nés au Brésil, les budgets participatifs sont aujourd'hui répandus dans la majorité des pays de l'OCDE. Par exemple, le Portugal comptait 118 budgets participatifs au niveau local en 2017, représentant un investissement de 91 millions EUR (euros) sur 2007-17.

L'étude de l'OCDE Cap sur les citoyens : La participation à l'appui de l'action et des services publics détaille une expérience de budget participatif en Corée. Le district Bouk-Gu du conseil métropolitain de Gwangju (463 000 habitants) a créé un portail électronique dédié au budget participatif comme un moyen d'augmenter l'implication des citoyens dans le processus budgétaire, en leur donnant accès à des informations détaillées et en améliorant la communication directe avec l'administration municipale (interactions de type questions-réponses) grâce aux technologies de l'information et de la communication. D'après le rapport d'évaluation du district, les citoyens ont soumis 378 propositions grâce au budget participatif. Quelques $70 \%$ ont été prises en compte dans la proposition budgétaire finale soumise à l'assemblée délibérative du district Bouk-Gu. La majorité des suggestions concernaient l'amélioration des services publics locaux.

Sources : (OCDE, 2017[15]) ; (Choi, 2009 $\left.9_{[16]}\right]$.

En ce qui concerne les besoins des services au sein de l'entité publique - services requérants, leur identification adéquate nécessite une bonne coordination avec les services chargés de la rédaction des marchés. D'après les missions exploratoires, la coordination entre les services requérants et ceux en charge de la rédaction des marchés est parfois limitée, ce qui impacte la façon dont les besoins sont formulés et traduits dans les documents d'appel d'offres. En effet, la collecte et l'analyse des besoins spécifiques de chaque service requérant ne doit pas se limiter à une démarche administrative, elle doit également comprendre des entretiens pour obtenir des renseignements complémentaires sur les besoins $\left(\mathrm{OCDE}\right.$, à paraître $\left.{ }_{[17]}\right)$. L'Algérie gagnerait donc à inciter les services en charge de la rédaction des documents d'appel d'offres à améliorer la coordination avec les services requérants.

\subsubsection{Renforcer l'analyse des marchés pour améliorer l'efficience du système de passation des marchés publics}

\section{Améliorer la conduite des études de marchés pour les services contractants}

Les études de marché permettent l'identification des capacités du marché en termes de volumes, de qualités et de paramètres techniques, afin de concevoir et rédiger des cahiers des charges et des documents d'appels d'offres adaptés à la réalité du marché, ce qui conditionne donc la bonne exécution de ce dernier. Par ailleurs, les études de marché permettent d'étudier l'opportunité d'utiliser les marchés publics comme levier stratégique pour atteindre des objectifs de politiques publiques, tels que le développement durable, l'accès des PME aux marchés publics ou encore l'innovation (voir chapitre 5). Enfin, elles permettent de réduire les asymétries d'information avec les fournisseurs et donc d'améliorer l'efficience du système en obtenant un meilleur rapport qualité prix (OCDE, 2017 [18]). En effet, les services contractants utilisent généralement les informations collectées lors des études de marché pour mettre en place leur stratégie d'achat et décider des modalités de passation du marché public (choix de la procédure, rédaction du cahier des charges, etc.) (OCDE, 2018[19]). 
Les études de marché doivent être conduites, même en l'absence d'obligation légale, pour toute procédure d'achat, sur la base de besoins formulés en termes de performances ou d'exigences fonctionnelles. Elles permettent de recueillir des éléments clés sur la structure du marché, les prix, les différents produits ou solutions disponibles ainsi que leurs substituts, y compris les innovations récentes (OCDE, 2016[20]). L'encadré 4.2 donne plus de détails sur les éléments à intégrer dans les études de marchés.

\section{Encadré 4.2. Les éléments à intégrer dans les études de marché}

Une bonne compréhension des conditions de marché et des fournisseurs potentiels est essentielle à l'efficacité de la commande publique. Les études de marché sont une analyse détaillée qui comprend non seulement les aspects prix, mais également les caractéristiques fonctionnelles des biens, services ou travaux, les conditions de livraison et la durée de vie des produits. Les éléments suivants sont à intégrer dans les études de marché :

- Les caractéristiques principales du marché : nombre de fournisseurs, pourcentage de PME sur ce segment, et part de marché des différents acteurs (nationaux ou internationaux selon les cas).

- Les caractéristiques principales des fournisseurs en termes de capacité à intégrer dans leurs offres des objectifs de politiques publiques (objectifs environnementaux, sociaux, d'accès des PME, d'innovation, etc.).

- La taille du marché et le volume de l'achat programmé par rapport à la taille du marché.

- Les caractéristiques influençant le degré de concurrence : produits simples ou standardisés, faiblesse ou absence de barrières à l'entrée, maturité des produits/services, intensité de l'innovation.

- Les caractéristiques des fournisseurs et leur structure de coût. Les prix pratiqués auprès des autres acheteurs (non publics) sont un élément d'information essentiel. Une comparaison avec les prix qui prévalent dans le cadre de transactions « $B$ to $B$ » (transactions commerciales entre entreprises privées) est nécessaire.

- Les évolutions récentes des prix, et les prix prévalent sur des marchés voisins, c'est-à-dire ceux des produits de substitution.

- Les appels d'offres récents concernant les mêmes produits ou services, ou des produits ou services similaires.

Les études de marché doivent être conduites par des experts qualifiés en matière d'achat public et/ou des experts sectoriels, disposant de suffisamment de temps et de ressources. Le cas échéant, le service contractant peut recueillir des informations auprès d'organismes publics ayant acheté des biens, services ou travaux similaires à ceux qu'il projette d'acquérir. Les études de marchés fournissent des indications précieuses qui facilitent, le cas échéant, la détection des schémas de collusion entre fournisseurs.

Source : (OCDE, 2014[21]).

De nombreux pays de l'OCDE mettent à la disposition des services contractants des lignes directrices ou des guides d'analyse des marchés afin de les aider à formuler des études de marché plus pertinentes et structurées. Par exemple en Australie, l'État de Queensland a développé un guide spécifique pour l'analyse des marchés du gouvernement de l'État de Queensland. Se référant aux cinq forces de Porter pour construire une approche structurée de l'analyse des marchés et comprendre les dynamiques compétitives, ce guide reprend des éléments de marketing achat pour aider les services contractants à construire une stratégie d'achat optimale. Il comprend des sections détaillées sur la structure des marchés, la dynamique concurrentielle entre les fournisseurs, les chaînes d'approvisionnement, les produits (biens 
ou services) de substitution, etc. (State of Queensland (Dpt of Housing and Public Works), 2018[22]). Ce guide comprend des exemples, tels que celui présenté dans l'encadré 4.3.

\section{Encadré 4.3. Guides d'analyse des marchés australiens, conclusions principales d'une étude de marché pour un produit électronique (hypothétique)}

\section{Structure du marché}

Quatre fournisseurs/producteurs dominent le marché et représentent $60 \%$ des achats publics. Des distributeurs représentent en outre $15 \%$ des achats publics. L'assemblage du produit est presque toujours réalisé à l'étranger.

\section{Concurrence}

Le prix est au cœur de la dynamique concurrentielle avec une pression à la baisse (marché mature). Malgré l'existence de différentes marques, il y a peu de différentiation produit réelle. Un cycle de vie court des produits entraîne de fortes réductions sur les modèles existants à partir d'un an après leur sortie. II existe des économies d'échelle importantes dans la distribution qui se fait via des grands réseaux nationaux. Certains fournisseurs mettent l'accent sur les services afin de protéger leur marge.

\section{Chaîne d'approvisionnement}

Il existe des risques de défauts critiques de certains composants clés, dont l'assemblage est réalisé à l'étranger. Les fournisseurs-revendeurs ajoutent une forte valeur ajoutée aux produits via les services associés qu'ils proposent. La plupart des fournisseurs font appel aux mêmes sous-traitants locaux pour les services de maintenance/réparation.

\section{Substituts}

II existe peu de substituts réels. Une tendance se développe, qui consiste à acheter un service à la place du produit physique.

\section{Le service contractant en tant qu'acheteur}

Le service contractant est un " petit » client comparé à l'ensemble des ventes nationales (moins de $0.1 \%$ ) et un client de taille moyenne au sein des achats gouvernementaux de ce produit ( 0.5 à $2 \%$ ). Le service contractant a des besoins avancés en termes de gestion de clientèle. Le service contractant ne sera pas perçu comme un client stratégique par la plupart des fournisseurs.

\section{Facteurs environnementaux et sociaux}

Le processus de fabrication exige de fortes dépenses en énergie et en eau. Les produits usagés présentent un risque de contamination environnementale et exigent un traitement approprié.

Par ailleurs, tous les fournisseurs manquent de transparence sur leur chaîne d'approvisionnement, notamment concernant la conduite responsable des entreprises. Certains fournisseurs ont entamé des démarches afin de s'adapter à des changements législatifs anticipés dans ce domaine.

Source : (State of Queensland (Dpt of Housing and Public Works), 2018[22]).

Par ailleurs, les lignes directrices sectorielles ou les guides spécialisés publiés dans les pays de l'OCDE à destination des acheteurs publics comprennent fréquemment des éléments indispensables pour la conduite d'une étude de marché. Par exemple, le gouvernement écossais met à disposition des services 
contractants un guide spécialisé dans le domaine des achats de services de soins à domicile et d'aide sociale (Scottish Government, 2016[23]). En France, la Direction des affaires juridiques du ministère de l'Économie et des Finances met à disposition une multitude de guides spécialisés qui permettent aux acheteurs publics de mieux préparer leurs études de marchés. Ces guides présentent des informations sur la structure du marché et proposent des définitions et des solutions techniques indispensables à la bonne compréhension du marché. Par exemple, il existe un guide sur l'efficacité énergétique dans les bâtiments (exemple d'un établissement scolaire) ou sur l'achat public de produits et de prestations d'entretien des espaces verts (Ministère de l'Economie et des Finances, France, 2019[24]).

En Algérie, le DMP ne prévoit pas d'articles consacrés à l'analyse du marché. Par ailleurs, aucune ligne directrice n'est disponible à ce sujet. En termes de renforcement des capacités, seul un module du diplôme d'études supérieures spécialisées (DESS) de l'Institut supérieur de gestion et de planification (ISGP)aborde le thème de "la maturation des commandes comme préalable au lancement de la procédure de passation des marchés ».

Lors des missions exploratoires de l'OCDE, la faiblesse de la phase d'étude de marché et des études préparatoires dans les marchés publics en Algérie a été identifiée comme l'une des plus grandes lacunes du système de passation des marchés publics. Une maturation insuffisante des projets, ou l'inadéquation des spécifications techniques par rapport aux besoins et/ou aux capacités du marché sont des problèmes fréquents. Ces problèmes résultent souvent d'insuffisances dans l'analyse du marché par les services contractants, en effet, l'étude de marché n'est pas toujours formalisée, elle se limite généralement à l'analyse des prix issus des procédures antérieures et à des recherches sur internet.

L'Algérie gagnerait donc à renforcer les études de marché conduites par les services contractants. Outre le renforcement des capacités des acheteurs publics, qui pourrait comprendre des actions de formation spécifiques en matière d'analyse de marchés, l'Autorité de régulation des marchés publics (ARMP), lorsqu'elle sera mise en place, pourrait élaborer des lignes directrices ou un guide pratique en matière d'études de marché. Ces lignes directrices, qui gagneraient à être publiées et disponibles en ligne, doivent fournir une assistance méthodologique et des exemples pratiques aux agents des services contractants. L'ARMP pourrait les compléter par des guides spécifiques par secteur (travaux publics, technologies de l'information, etc.), dont l'élaboration devrait se faire conjointement avec les ministères sectoriels concernés, et qui devraient comprendre une section dédiée aux fournisseurs et à l'environnement économique du secteur. Ils devraient aborder également les spécificités techniques et réglementaires du secteur, dont la compréhension est indispensable à la conduite d'une étude de marché approfondie.

Enfin, la mise en place du portail électronique des marchés publics pourrait être l'occasion de mettre sur pied une bibliothèque d'études de marché à disposition des services contractants. Les acheteurs publics pourraient ainsi avoir accès à des études de marché récentes concernant les fournitures, les services ou même les travaux correspondants à leurs besoins.

\section{Mettre en place des dialogues plus réguliers entre les services contractants et le secteur privé}

Afin de pouvoir rédiger des cahiers des charges et documents d'appel d'offres adaptés á la réalité du marché, il est parfois nécessaire pour les entités publiques de consulter les fournisseurs potentiels. Ce dialogue est indispensable pour réaliser les études de marché et ajuster la stratégie achat des services contractants. Cependant, les services contractants doivent communiquer de façon équitable et transparente avec les fournisseurs potentiels afin de ne pas accorder d'avantage indu à l'un ou à plusieurs d'entre eux (OCDE, 2017[18]).

Différents outils peuvent permettre de renforcer le dialogue avec les fournisseurs potentiels tout en assurant l'équité et la transparence du processus, tels que la publication de demandes d'information ou de sollicitations préalablement à la phase d'appel d'offres, ou la mise en place de questionnaires pour 
recueillir des informations auprès des fournisseurs. La communication avec les soumissionnaires par l'entremise d'une plateforme web est également une bonne pratique de plus en plus répandue parmi les pays membres de l'OCDE (OCDE, 2016[25]). Par exemple, la Grèce utilise son registre électronique de fournisseurs pour constituer un panel ad hoc de fournisseurs dans le cadre des consultations lors de la préparation d'un appel d'offres. Le Mexique publie des versions préliminaires des cahiers des charges sur sa plateforme web Compranet en amont d'un appel d'offres afin de recueillir des commentaires des opérateurs économiques (OCDE, 2018[26]).

Un autre outil pour renforcer le dialogue est l'organisation de rencontres avec des associations de fournisseurs ou des fournisseurs potentiels sous la forme de panel ad hoc ou de journées portes ouvertes. Ces rencontres existent dans de nombreux pays de l'OCDE, comme par exemple en Nouvelle-Zélande, en Italie ou en Grèce. Elles prennent parfois la forme d'entretiens organisés entre les acheteurs publics des principaux pouvoirs adjudicateurs et les fournisseurs intéressés par des catégories d'achats spécifiques (OCDE, 2018[6]). Les entités adjudicatrices préparent souvent des comptes rendus ou procèsverbaux des échanges avec les fournisseurs. (OCDE, 2018[26]). L'encadré 4.4 décrit le processus de consultation auprès des opérateurs économiques mis en place en Italie par la principale centrale d'achat public Consip SpA.

\section{Encadré 4.4. Questionnaires de consultation de marché et rencontres fournisseurs en Italie}

La consultation du marché débute par la publication de questionnaires spécifiques à chaque projet d'achat sur la plateforme de passation électronique des marchés publics et le site internet de la Consip, la principale centrale d'achat public italienne. L'objectif des questionnaires est de recueillir des informations auprès des fournisseurs concernant une catégorie d'achat, et ainsi d'obtenir une meilleure connaissance des caractéristiques du marché, de garantir la participation des entreprises et d'assurer la diffusion de l'information. Les questionnaires sont en ligne jusqu'à la publication de l'avis d'appel d'offres.

Des réunions avec les opérateurs économiques peuvent avoir lieu à leur demande. Un minimum de deux employés de Consip SPA (chef de projet et gestionnaire de catégorie) doivent y participer. Pendant la réunion, Consip présente le questionnaire d'étude de marché spécifique au projet d'achat et distribue un exemplaire de son code d'éthique. Le questionnaire est ensuite complété par l'ensemble des participants à la réunion.

Des questions peuvent être posées pendant la réunion ; toutefois, aucune information supplémentaire par rapport à celles publiées en ligne n'est fournie, de sorte que les fournisseurs qui ne participent pas à la réunion ne soient pas désavantagés. La stratégie d'appel d'offres (si elle est déjà établie) n'est pas discutée et aucune comparaison entre les soumissionnaires potentiels n'a lieu.

Source : (OCDE, 2013[27]).

En Algérie, les missions exploratoires de l'OCDE ont permis d'établir que les études de marché n'impliquent généralement pas de mécanismes formalisés de dialogue avec les fournisseurs potentiels. Des rencontres informelles ont parfois lieu entre acheteurs publics et fournisseurs. L'une des raisons pour lesquelles les services contractants n'ont pas recours aux mécanismes formalisés de dialogue avec les fournisseurs est qu'ils craignent que ces pratiques soient interprétées comme des atteintes à l'intégrité. Cependant, sans ces mécanismes de dialogue avec les opérateurs économiques, les agents en charge de la passation des marchés publics et/ou les experts techniques sectoriels ne peuvent pas acquérir une connaissance et une compréhension globale du marché. La mise en place de dialogues formalisés entre les services contractants et le secteur privé, ainsi que d'un processus de demande d'informations auprès 
du marché, notamment via les rencontres avec les fournisseurs potentiels, pourraient améliorer l'analyse du marché et donc l'efficience du système, en favorisant une bonne compréhension du marché par les services contractants et une meilleure préparation des documents d'appel d'offres.

La mise en place de tels dialogues formalisés avec les opérateurs économiques exige cependant un changement de culture pour les agents des services contractants. Afin de préserver l'intégrité de la passation du marché et l'équité entre fournisseurs, ces dialogues doivent prévoir les garanties nécessaires. En particulier, les éléments essentiels de chaque rencontre doivent être consignés et disponibles en cas d'audits ou de vérifications ultérieures. À plus long terme, l'Algérie pourrait envisager d'utiliser son système de passation électronique des marchés publics dans le cadre des consultations avec les fournisseurs pour diffuser des demandes d'information, annoncer les rencontres avec les fournisseurs, publier leurs comptes rendus, etc.

\subsubsection{Consolider la maturation des projets et les études de maturation}

Dans le cas des grands projets d'équipement et d'infrastructures, la phase de conception comprend la préparation de la documentation d'études et des estimations financières, qui sont parfois confiées à des bureaux d'étude lorsque l'entité publique n'a pas la capacité technique pour les réaliser. La qualité des études techniques et l'élaboration de la documentation d'étude est déterminante pour la bonne exécution du projet, en particulier en termes de qualité, mais aussi en termes de délais et de coût total du projet (OCDE, 2017[28]).

En Algérie, la Caisse nationale d'équipement pour le développement (CNED), dont le rôle est décrit dans l'encadré 4.5, est la seule autorité extérieure au maître d'ouvrage à se prononcer sur l'opportunité des grands projets d'infrastructure, sur leur faisabilité et sur le degré d'aboutissement des études de maturation. Depuis 2010, la CNED met en œuvre le Guide de maturation des grands projets d'infrastructure économique et sociale, qui a introduit dans la pratique algérienne la maturation des grands projets en trois phases : les études d'identification, de faisabilité et de préparation à la réalisation.

\section{Encadré 4.5.Grands projets d'infrastructure : les prérogatives de la CNED}

La CNED est un organisme auprès du ministère des Finances. À chacune des trois étapes de la maturation d'un grand projet d'infrastructure, la CNED remet un avis motivé sur les études préparatoires, demandant si nécessaire des compléments d'information ou des corrections au maître d'ouvrage (ministères sectoriels le plus souvent) et aux bureaux d'études. La CNED intervient dès lors que le montant de l'autorisation de programme dépasse 20 milliards DZD (dinars algériens, environ 150 million EUR).

Avant le lancement du projet, la CNED s'assure que l'estimation du coût d'investissement découlant des études techniques n'est pas de nature à remettre en cause la rentabilité économique du projet et ne conduit pas à un impact supplémentaire important sur le Budget de l'État. La CNED peut, si besoin, exiger des études complémentaires avant de donner un avis favorable au lancement du projet. L'avis favorable de la CNED n'est cependant pas obligatoire pour le lancement des projets d'infrastructure, ce qui en fait une instance à caractère consultatif du point de vue des maîtres d'ouvrage.

Dans la phase de réalisation des projets, la CNED est également en charge du suivi des grands projets d'infrastructure, afin notamment d'identifier et de prévenir les dérapages dans la réalisation du projet qui augmentent son coût par rapport au budget initial. Elle assure un suivi trimestriel de la réalisation des grands projets sur la base du devis quantitatif et estimatif issu des études de préparation de réalisation de l'investissement.

Source : (Caisse nationale d'équipement pour le développement (CNED), 2008[14]). 
À chacune de ces étapes, la CNED remet un avis motivé sur les études réalisées par les maîtres d'ouvrage (ministères sectoriels) et les bureaux d'études. D'après le Guide de maturation, ces principales étapes se déclinent de la manière suivante :

- Les études d'identification visent à confirmer la pertinence du projet pour l'économie nationale et à préparer les termes de référence des études de faisabilité.

- Les études de faisabilité donnent lieu à la réalisation d'une analyse coûts-bénéfices du projet et confirment sa viabilité technique et environnementale. Une première estimation prévisionnelle des coûts est réalisée.

- Les études de préparation arrêtent la configuration technique détaillée (avant-projet détaillé), affinent l'estimation des coûts et définissent les modalités de réalisation du projet. Elles donnent lieu à la préparation des dossiers d'appel d'offres (Caisse nationale d'équipement pour le développement (CNED), 2008[14]).

Cette chronologie en trois étapes a commencé à s'appliquer dans la pratique en 2012-14, entraînant une certaine amélioration dans la conduite des projets, les maîtres d'ouvrages commençant à prendre en compte les prescriptions de la CNED (Ministère des Finances, Algérie, 2014[29]). Cependant, le rôle de la CNED dans la maturation des projets et sa consultation obligatoire à chaque étape ne sont établis par aucune loi ni aucun texte réglementaire. Par exemple, le décret exécutif $n^{\circ}$ 04-162 portant statut, organisation, missions et attributions de la CNED date de juin 2004 : il est donc antérieur au Guide de maturation de 2010. II stipule seulement que la CNED « se prononce sur la faisabilité économique, technique, sociale et financière des grands projets d'équipement et donne un avis avant leur inscription à la nomenclature des investissements publics » (JORA, 2004[30]).

Les missions exploratoires de l'OCDE ont montré que la faible qualité des nombreuses études de maturation est l'un des principaux facteurs entraînant des surcoûts et des retards dans les projets d'infrastructure et de travaux publics. Ces déficiences engendrent un recours massif aux avenants, que ce soient des avenants de régularisation des travaux supplémentaires nécessaires mais réalisés sans assise contractuelle, ou des avenants dont la signature est nécessaire avant la réalisation de travaux complémentaires, non identifiés préalablement dans le contrat initial.

Le constat de lacunes persistantes dans la préparation des grands projets d'infrastructures est partagé par les autorités. En 2014, le ministère des Finances considérait que " certaines insuffisances, voire lacunes, persistent particulièrement en matière de préparation et d'exécution des projets, ce qui se traduit systématiquement par un allongement des délais et une réévaluation des coûts de réalisation ». S'agissant de la maturation des projets, la CNED soulignait notamment l'insuffisance de certaines études, l'inadéquation du délai de réalisation avec l'importance du projet, et le lancement de projets sur la base d'études non maturées (Ministère des Finances, Algérie, 2014[29]).

D'après les missions exploratoires de l'OCDE, les déficiences des études de maturation et de la préparation des projets peuvent s'expliquer par deux facteurs :

1. Les études techniques de faisabilité et de préparation de l'investissement (telles que l'avant-projet détaillé) sont généralement réalisées par des bureaux d'études. Leurs études techniques sont parfois imprécises ou de qualité insuffisante.

2. Les prérogatives de la CNED sont insuffisantes en matière de maturation des projets. Son avis favorable n'étant pas obligatoire pour le lancement d'un grand projet d'infrastructure. En effet, en tant qu'organisme subordonné au ministère des Finances, la CNED transmet ses avis à ce dernier, qui les fait suivre aux ministères sectoriels, lesquels exercent le plus souvent la fonction de maître d'ouvrage pour les projets d'envergure nationale (Caisse nationale d'équipement pour le développement (CNED), 2008[14]). En outre, il n'est pas du ressort de la CNED de se prononcer sur les solutions techniques choisies par la maîtrise d'ouvrage, sa mission est centrée sur la rentabilité économique et sociale du projet et le réalisme de l'estimation prévisionnelle des coûts. 
Cependant, en pratique, des solutions techniques différentes peuvent engendrer des coûts d'investissement différents, et il peut être difficile de séparer nettement l'évaluation financière et économique d'une part, et les caractéristiques techniques d'un projet, d'autre part. Ainsi, malgré la maturation des grands projets en trois phases et l'expertise de la CNED, le surdimensionnement par rapport aux besoins demeure un problème récurrent dans les grands projets d'infrastructures (Caisse nationale d'équipement pour le développement (CNED), 2008[14]).

Au vu des responsabilités de la CNED dans la maturation des grands projets d'infrastructure, le gouvernement algérien pourrait renforcer les prérogatives de la CNED. Les dispositions clés du Guide de maturation de 2010, en particulier les trois étapes de maturation des projets d'infrastructure (études d'identification, de faisabilité et de préparation à la réalisation), devraient être intégrées au cadre réglementaire. L'avis favorable de la CNED concernant les études de maturation devrait être obligatoire pour que le maître d'ouvrage lance l'étape suivante. Le cadre réglementaire devrait donc préciser que l'avis favorable de la CNED est obligatoire avant le lancement de tout projet d'infrastructure économique et sociale de son ressort.

Afin de consolider le rôle institutionnel de la CNED, le gouvernement pourrait la doter du statut d'organisme indépendant, à l'instar par exemple de la Commission de régulation de l'électricité et du gaz ou d'autres organismes indépendants comparables sans tutelle ministérielle. En outre, il convient d'élargir le périmètre d'intervention de la CNED : elle devrait pouvoir se prononcer sur les solutions techniques retenues par le maître d'ouvrage, au-delà de la seule opportunité des projets et des aspects économiques et financiers (risques de dérapages budgétaires et en termes de délai).

Enfin, avant de donner son avis favorable, la CNED devrait conduire davantage d'études complémentaires afin de mettre en lumière, le cas échant, les insuffisances des études de préparation de réalisation de l'investissement (avant-projet détaillés, etc.). Cela inciterait les maîtres d'ouvrage à investir davantage dans la qualité des études de maturation.

\subsection{Des documents d'appel d'offres adaptés à la réalité du marché}

Les critères de qualification et de sélection des fournisseurs contenus dans les cahiers des charges ont un impact direct sur le niveau de concurrence et la bonne exécution des marchés. En effet, le cahier des charges permet de sélectionner la meilleure offre possible en fonction de l'objet du marché. Des critères de sélection inadéquats peuvent empêcher l'attribution du marché au fournisseur ayant proposé la meilleure offre, entraînant des surcoûts ou même une mauvaise exécution du contrat (Union Européenne, $\left.2014_{[7]}\right)$. Les critères de qualification disproportionnés peuvent favoriser un candidat, ce qui va à l'encontre du principe d'égalité d'accès à la commande publique. Les critères de qualification et de sélection sont également un instrument privilégié pour la prise en compte d'objectifs de politiques publiques (OCDE, 2018[19]).

Par ailleurs, dans le cadre de certains contrats, notamment les contrats pluriannuels, les cahiers des charges doivent faire référence aux conditions de révision des prix, qui seront reprises dans les clauses de révision des prix des contrats. Des conditions de révision des prix adéquates et transparentes sont cruciales pour la bonne exécution des contrats, et reposent notamment sur la disponibilité d'indices de prix pertinents (SIGMA, 2016[31]). 


\subsubsection{Encourager l'utilisation de critères de qualification et de sélection adéquats, au- delà du seul critère du prix}

\section{Des critères de qualification qui ne doivent pas être restrictifs}

Les critères de qualification garantissent la capacité d'un opérateur économique à exécuter le contrat. Ils permettent aux services contractants d'exiger que les soumissionnaires présentent un minimum de capacités techniques, financières et professionnelles (OCDE, 2011[32]). Les soumissionnaires ne présentant pas ces capacités minimales ou qui présentent une offre non conforme aux caractéristiques minimales requises ne peuvent pas soumissionner. Cependant, des critères de qualification excessivement restrictifs peuvent représenter un obstacle pour l'accès des PME aux marchés publics (Commission Européenne, 2008[33]), et par conséquent diminuer le degré de compétition dans les appels d'offres (voir chapitre 5).

En Algérie, le service contractant doit vérifier les capacités humaines, financières et techniques des soumissionnaires, lesquels doivent soumettre «tout document permettant d'évaluer les capacités des candidats, des soumissionnaires, et le cas échéant, des sous-traitants » (art. 67 du DMP). Le cahier des charges peut exiger la production d'un certificat de qualification ou d'un agrément obligatoire par les soumissionnaires. C'est systématiquement le cas en matière de travaux publics, de travaux forestiers, ou dans le secteur des ressources en eau. Cependant aucune information n'a pu être collectée quant aux difficultés pour les opérateurs économiques d'obtenir les certificats de qualification ou agréments. Par ailleurs, dans la pratique, les missions exploratoires de l'OCDE ont relevé que les services contractants se fondent parfois sur les trois derniers bilans pour estimer les capacités humaines et financières d'un soumissionnaire, ou exigent un minimum de chiffre d'affaires.

Des dispositions sont prévues pour faciliter l'accès des PME aux marchés publics, et donc intensifier la concurrence, ce qui correspond aux bonnes pratiques internationales et contribue à l'efficacité de la commande publique (voir chapitre 5). Des lignes directrices seraient toutefois nécessaires afin de préciser ces dispositions et d'assurer leur application uniforme par les services contractants. Elles devraient préciser que la référence à des normes techniques ou des standards doit être accompagnée de la mention " ou équivalent», qui ouvre la porte à la soumission de variantes, c'est-à-dire des offres techniques alternatives qui répondent au besoin mais ne satisfont pas nécessairement aux standards définis dans le cahier des charges (Union Européenne, 2014 ${ }_{[7]}$ ). Ces lignes directrices pourraient également donner des indications et des exemples concrets aux services contractants afin de les guider dans le choix des exigences financières utilisées comme critères de qualification. Il est en effet essentiel que ces exigences soient proportionnelles à l'objet du contrat et ne représentent pas des barrières excessives à la participation des PME.

\section{Vers un recours plus fréquent à l'offre économique la plus avantageuse et au coût global d'utilisation}

Les critères de sélection ou d'attribution permettent d'évaluer les offres et d'attribuer le marché à l'opérateur économique ayant présenté la meilleure offre. En plus du critère relatif au coût/prix, ces critères peuvent porter sur des aspects relatifs à la qualité, aux caractéristiques techniques, au service aprèsvente, mais également aux aspects environnementaux et sociaux (OCDE, 2011[32]). 
Dans la pratique internationale, il existe deux méthodes pour la sélection des offres dans le cadre d'une procédure de marché public :

1. L'offre la moins-disante: le marché est attribué à l'offre présélectionnée (suite à la phase de présélection) la moins chère.

2. Le meilleur ratio prix/efficacité (ou rapport qualité/prix), qui permet de prendre en compte d'autres critères de sélection que le prix. Cette méthode (anciennement désignée offre économiquement la plus avantageuse) présente plusieurs avantages par rapport l'offre la moins-disante car elle permet de prendre en compte de multiples critères de sélection (tableau 4.1).

\section{Tableau 4.1. Inconvénients de l'utilisation du seul critère du prix pour la sélection des offres}

\begin{tabular}{l|l|l|l}
\hline $\begin{array}{c}\text { Méthode de } \\
\text { sélection }\end{array}$ & $\begin{array}{c}\text { Avantages/inconvénients en } \\
\text { termes d'innovation }\end{array}$ & $\begin{array}{c}\text { Avantages/inconvénients en } \\
\text { termes de concurrence }\end{array}$ & $\begin{array}{l}\text { Avantages/inconvénients en termes de } \\
\text { critères sociaux ou environnementaux }\end{array}$ \\
\hline $\begin{array}{l}\text { Offre la } \\
\text { moins-disante }\end{array}$ & $\begin{array}{l}\text { Ne permet pas au pouvoir } \\
\text { adjudicateur de valoriser l'innovation }\end{array}$ & $\begin{array}{l}\text { Décision d'attribution prévisible : } \\
\text { risque d'ententes anti- } \\
\text { concurrentielles }\end{array}$ & $\begin{array}{l}\text { Pas de prise en compte de critères sociaux } \\
\text { ou environnementaux }\end{array}$ \\
\hline $\begin{array}{l}\text { Rapport } \\
\text { qualité/prix (ou } \\
\text { prix/efficacité) }\end{array}$ & $\begin{array}{l}\text { Permet au pouvoir adjudicateur de } \\
\text { valoriser les éléments innovants } \\
\text { d'une offre }\end{array}$ & $\begin{array}{l}\text { Décision d'attribution moins } \\
\text { prévisible du fait de la multiplicité } \\
\text { des critères }\end{array}$ & $\begin{array}{l}\text { Possibilité de critères environnementaux } \\
\text { (certificats, écolabels, etc.) ou sociaux (tels } \\
\text { que la responsabilité sociale des entreprises } \\
\text { [RSE]) }\end{array}$ \\
\hline
\end{tabular}

Sources : (OCDE, 2012[34] ; OCDE, 2011[32]).

Malgré ses limites (voir tableau 4.1) le choix de l'offre moins-disante, y compris avec l'exigence d'une note technique minimale, peut être pertinente dans le cas d'achats de produits fortement standardisés et aux caractéristiques préétablies. Elle doit en revanche être exclue dès lors que les besoins des pouvoirs adjudicateurs présentent un certain degré de complexité (Crown Agents, 2018[35]).

Dans la méthode de l'offre économique la plus avantageuse, le poids de chacun des critères est généralement déterminé par un système de pondération qui doit être précisé dans le cahier des charges, et va guider les opérateurs économiques dans la préparation de leurs offres. La pondération des critères doit être calibrée avec soin et refléter les objectifs stratégiques que veulent atteindre les entités publiques, afin de sélectionner l'offre qui propose la meilleure valeur ajoutée (OCDE, à paraître $\left.{ }_{[36]}\right)$. Certains pays ont développé des lignes directrices à destination des services contractants concernant la pondération des critères de sélection des marchés de fournitures afin de refléter des objectifs stratégiques nationaux. Le tableau 4.2 présente les lignes directrices de pondération des critères d'attribution des marchés de fournitures élaborées par le ministère de l'Administration publique du Mexique où le prix représente, au maximum, la moitié des points attribués.

\section{Tableau 4.2. Lignes directrices pour l'attribution des marchés de fournitures au Mexique}

\begin{tabular}{|c|c|c|}
\hline & \multirow{6}{*}{$\begin{array}{l}\quad \text { Critères d'attribution } \\
\text { Caractéristiques techniques des biens } \\
\text { Capacités du fournisseur } \\
\text { Expérience du fournisseur sur le marché } \\
\text { Performance dans l'exécution des contrats } \\
\text { Prix }\end{array}$} & Pondération \\
\hline \multirow[t]{4}{*}{ Critères techniques } & & 20-25 points \\
\hline & & 5-10 points \\
\hline & & 5-7.5 points \\
\hline & & $10-20$ points \\
\hline Critères de prix & & Maximum 50 points \\
\hline
\end{tabular}

Source :ministère de la Fonction publique, Mexique. 
En Algérie, la réglementation concernant les critères de sélection est conforme aux bonnes pratiques internationales. En effet, l'article 78 du DMP, qui spécifie les critères de sélection, est exhaustif et mentionne une série de critères pertinents pour choisir l'offre économiquement la plus avantageuse. II décrit des critères de sélection relatifs au coût (y compris le coût global d'utilisation) et hors coût (qualité, délais d'exécution ou de livraison, caractère fonctionnel, valeur technique, service après-vente et assistance technique, etc.). II permet également le recours à des critères de sélection portant sur les performances en matière sociale et de développement durable des fournisseurs (voir chapitre 5 sur la prise en compte des objectifs de politique publique dans les marchés publics). Sur cette base, les entités publiques utilisent trois méthodes différentes pour sélectionner le partenaire co-contractant dans le cadre d'un appel d'offres :

- le choix de l'offre la moins-disante en se basant uniquement sur le critère prix,

- le choix de l'offre la moins-disante parmi les offres ayant obtenu une note technique minimale,

- le choix de l'offre ayant la note totale la plus élevée sur la base de la pondération de plusieurs critères, lorsque le choix est essentiellement basé sur l'aspect technique des prestations.

L'Algérie ne dispose pas de données pour mesurer le recours aux différentes méthodes de sélection des offres. Cependant, d'après les discussions menées lors des missions exploratoires de l'OCDE avec différentes parties prenantes, il semble que les deux premières méthodes relatives à l'offre la moinsdisante soient dominantes dans l'attribution des marchés publics en Algérie. Cela fait du prix le facteur décisif de l'attribution, alors que la conformité aux spécifications techniques est évaluée sur une simple base réussite ou échec.

Le gouvernement algérien gagnerait à encourager les services contractants à utiliser d'avantage la méthode du ratio prix/efficacité en utilisant plusieurs critères de sélection, comme cela est prévu dans le cadre réglementaire, et ce, notamment lorsque les besoins des services contractants ont un certain degré de complexité. Cette mission devrait être confiée à l'ARMP prévue par le DMP. Une fois opérationnelle, l'ARMP pourrait développer un guide ou des lignes directrices à destination des services contractants concernant l'utilisation de différents critères de sélection. Ces lignes directrices pourraient être développées pour chaque grande catégorie d'achats (fournitures, services informatiques et technologies de l'information, travaux publics, etc.) et gagneraient à être accompagnées d'actions de formations appropriées à destination des acheteurs publics, fondées sur des bonnes pratiques internationales et algériennes.

L'évaluation financière de l'offre peut se faire en se fondant sur une analyse du coût global d'utilisation, qui prend en compte le coût d'utilisation d'un produit tout au long de son cycle de vie. L'encadré 4.6 décrit les différents coûts directs et indirects qui doivent être pris en compte pour le calcul du coût global d'utilisation. 


\section{Encadré 4.6. Le calcul du coût global d'utilisation}

En plus du prix d'achat, le calcul du coût global d'utilisation permet l'intégration des différents coûts d'exploitation, de maintenance et de désinstallation/recyclage afin d'assurer une réelle comparabilité des offres. Ces coûts incluent :

1. les coûts d'acquisition : prix d'achat, coûts d'installation, assurance, formation,

2. les coûts opérationnels : consommation d'énergie, consommables et frais de maintenance ou d'exploitation,

3. les coûts liés à la fin de vie, tels que les coûts de collecte et de recyclage.

Dans certaines approches intégrant des critères environnementaux, les coûts imputés aux externalités environnementales (comme par exemple les émissions de gaz à effet de serre) sont pris en compte, à condition que leur valeur monétaire puisse être établie.

Source : (SIGMA, 2017[37]).

Comme évoqué précédemment, le cadre réglementaire régissant les marchés publics en Algérie prévoit explicitement l'utilisation du coût global d'acquisition comme critère de sélection, ce qui est considéré comme une bonne pratique internationale. Cependant, dans la pratique, son utilisation est très limitée, mis à part dans le domaine de la santé où les coûts opérationnels sont également analysés. D'après les parties prenantes interrogées lors des missions exploratoires de l'OCDE, cette utilisation limitée est liée à un manque de capacité des entités publiques pour utiliser ce critère. L'Algérie gagnerait donc à développer des lignes directrices pour sensibiliser les entités publiques au concept du coût global d'acquisition. Par ailleurs des formations et actions de sensibilisation pourraient également être prévues sur ce sujet.

\subsubsection{Insérer des clauses de révision des prix pour assurer l'équilibre des contrats publics}

\section{Mettre à jour et publier en ligne les indices de révision des prix des contrats}

Les entités publiques et les opérateurs économiques pourraient être confrontés à des situations légitimes nécessitant la modification des prix fixés dans un contrat, en raison par exemple d'un changement du coût des approvisionnements d'un fournisseur, ou encore de circonstances imprévisibles survenues pendant l'exécution d'un marché. Ainsi, une hausse significative du prix des intrants pourrait dégrader la situation financière du fournisseur et nuire à la bonne exécution du contrat, alors qu'au contraire, une baisse des coûts d'approvisionnement qui serait captée par le fournisseur engendrerait des surcoûts pour les pouvoirs adjudicateurs (OCDE, 2018[19]). Les clauses de révision des prix sont particulièrement pertinentes pour certains types de contrat de longue durée, notamment lorsqu'ils portent sur des intrants dont les prix sont volatils (matière, main d'œuvre, autres coûts variables).

L'absence de clauses d'ajustement des prix peut représenter un risque pour les soumissionnaires, les décourageant à participer à un appel d'offres, ce qui impacte donc le niveau de concurrence. Ils pourraient ajouter une prime de risque à leur offre économique, faisant supporter le risque par les pouvoirs adjudicateurs via une augmentation du prix (OCDE, 2018[19]).

Les pays de l'OCDE ont introduit des clauses d'ajustement des prix dans leurs contrats publics, fondées sur une référence à des prix publics (Barème du titulaire du marché) ou à des indices de prix fiables et pertinents par rapport à l'objet du marché, publiés souvent par les instituts statistiques nationaux, comme par exemple en Espagne (encadré 4.7) (OCDE, 2018[19]). Afin de disposer de formules de révisions 
pertinentes et d'assurer un équilibre financier du contrat public, cette dernière méthode nécessite une actualisation et une publication régulières des indices de prix utilisés dans les formules de révision des prix, ainsi que la publication d'indices suffisamment détaillés en termes d'intrants, de biens et de services. La révision des prix sur Barème du titulaire peut notamment être envisagée lorsqu'aucun indice des prix n'est représentatif. Le choix de cette référence suppose que le pouvoir adjudicateur prenne certaines précautions puisque le titulaire fixe librement son prix, alors que la référence retenue pour une variation de prix à répercuter sur le prix d'un contrat ne devrait pas être laissée à la discrétion du seul titulaire (Ministère de l'Économie et des Finances (DAJ), 2013[38]) (Le Moniteur, s.d. [39]).

\section{Encadré 4.7. Indices de prix des matières et de main d'œuvre en Espagne}

En Espagne, les indices de prix des matières et de la main d'œuvre utilisés dans les formules de révision des prix des contrats publics sont publiés en ligne sur le site de l'Institut national de statistiques. Le calcul des indices mensuels des prix des matières, dont les formules sont approuvées par décret royal, est la responsabilité de l'Institut national de statistiques. II les diffuse sur son site web une fois les indices publiés au journal officiel et approuvés par une commission gouvernementale sur proposition du Comité supérieur des prix des contrats publics.

Source : (INE, s.d.[40]).

Le DMP prévoit des marchés à prix fermes et d'autres à prix révisables qui incluent des clauses de révision des prix basées sur des indices de prix officiels publiés au journal officiel et au Bomop (art. $97 a ̀$ 101 du DMP). Toutefois, les marchés dont les montants sont inférieurs aux seuils des procédures formalisées ne peuvent faire l'objet d'une révision des prix (art. 97 du DMP).

Les clauses de révision des prix sont particulièrement nécessaires en Algérie, où l'inflation est plus élevée que dans les pays de l'OCDE (graphique 4.3). Cependant d'après les missions exploratoires de l'OCDE, le recours aux marchés à prix ferme est souvent privilégié par les services contractants.

\section{Graphique 4.3. Taux d'inflation dans les pays de l'OCDE et en Algérie (2007-17)}

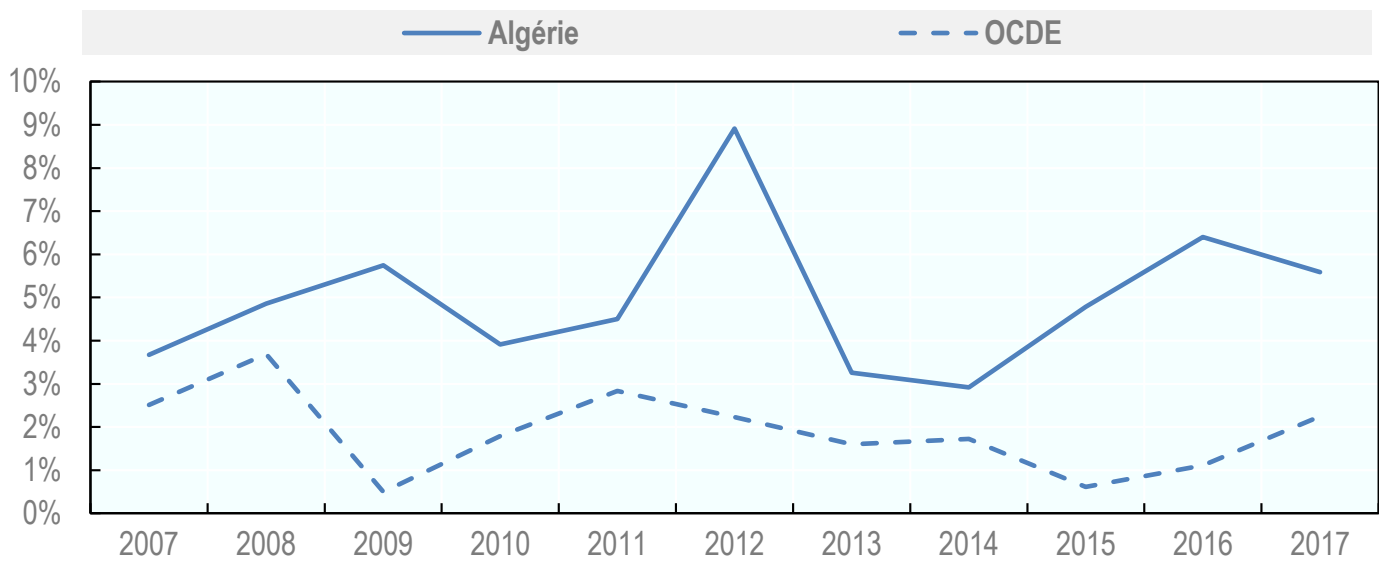

Note : Moyenne annuelle.

Sources : (Fonds Monétaire International, 2018[41] ; OCDE, 2019[42]) 
Par ailleurs, contrairement à la pratique des pays de l'OCDE, les indices de prix ne sont pas disponibles sur le site de l'Office national des statistiques (ONS). Ils sont établis par arrêté du ministre de l'Habitat, de l'Urbanisme et de la Ville ou, le cas échéant, par arrêté d'autres ministres, en fonction de leurs secteurs. D'après les analyses de l'équipe de l'OCDE, les catégories d'intrants ou de biens et services disposant d'indices de prix sont limitées et gagneraient à être actualisés plus fréquemment. À titre d'exemple, en mars 2019 les derniers indices des prix salaires et matières disponibles au journal officiel portaient sur le premier trimestre de l'année 2018 (JORA, 2004[30]). En outre, il semble que dans la pratique les seuls indices qui existent concernent le secteur de la construction. Par ailleurs, les indices des prix et matières sont publiés au journal officiel (disponible en format PDF), mais le format du journal officiel n'est pas adapté à une l'utilisation facile des indices par les opérateurs économiques et les services contractants (historique non disponible, format non exploitable directement pour l'utilisation des données).

L'Algérie gagnerait à publier des indices suffisamment détaillés et pertinents pour être utilisés dans les clauses de révision des prix dans les contrats publics. Cela exige de publier un plus grand nombre d'indices couvrant plus d'intrants, de biens et de services que ceux qui sont disponibles actuellement. En outre, il est essentiel que ces indices soient plus souvent mis à jour et disponibles en ligne de manière centralisée, y compris l'historique de l'indice sur une période longue. Des indices pertinents et suffisamment détaillés, disponibles gratuitement sur un site unique dans un format directement exploitable (Excel, etc.) stimuleraient sans doute l'usage des clauses de révision des prix par les services contractants et contribueraient à davantage de transparence sur les prix dans les marchés publics.

L'Algérie pourrait confier à l'ONS la centralisation et la publication sur son site de l'ensemble des indices de référence pour les formules de révision des prix utilisées dans les marchés publics. En effet, l'ONS dispose des compétences et outils nécessaires au traitement et à la publication régulière de ce type de données. Cela nécessitera, le cas échéant, une coopération entre les Ministères sectoriels concernés, y compris celui de l'Habitat, de l'Urbanisme et de la Ville, et l'ONS. La centralisation de la publication des indices par l'ONS n'empêche pas les différents ministres de continuer à publier les indices relatifs à leurs secteurs.

\subsection{Mettre en place des outils pour améliorer l'efficacité de la passation des marchés}

L'efficacité de la commande publique et la bonne utilisation des fonds publics sont des principes fondamentaux dans la plupart des systèmes de passation des marchés publics. Afin d'optimiser les dépenses, de réduire les doublons et d'accroître l'efficacité administrative de la passation des marchés publics, plusieurs pays, y compris ceux de l'OCDE, ont recours à des outils d'efficience tels que la mutualisation des achats, la mise en place d'accords-cadres et/ou la création de catalogues électroniques (OCDE, 2016[43]) (OCDE, 2018[26]).

\subsubsection{Encourager la mutualisation des achats publics pour bénéficier d'économies d'échelles}

L'agrégation de la demande et la mutualisation des achats sont des leviers majeurs d'efficacité de la commande publique. Les principaux arguments en faveur de la mutualisation des achats sont la réalisation d'économies grâce à un pouvoir de négociation accru auprès des fournisseurs et l'amélioration de l'efficacité administrative (réduction des doublons et des coûts de transaction pour les entités adjudicatrices et pour les fournisseurs) (OCDE, 2011[44]) (OCDE, 2018[6]). Toutefois, une mutualisation non maîtrisée et non réfléchie peut également représenter des risques, tels que la difficulté de satisfaire des besoins hétérogènes exprimés par les services contractants et un accès plus difficile pour les PME aux marchés publics en l'absence de stratégie d'allotissement (Sanchez-Graells et Herrera Anchustegui, 2016[45]). 
En pratique, l'agrégation de la demande peut se faire à des niveaux différents : par des groupements de commandes entre deux ou plusieurs entités publiques ayant des besoins homogènes, ou par la centralisation des achats à travers la création d'une structure ad hoc dont la mission principale est de passer des marchés publics aux profits de plusieurs entités adjudicatrices (graphique 4.4) (OCDE, 2016[46]).

\section{Graphique 4.4. Différents niveaux d'agrégation de la demande}

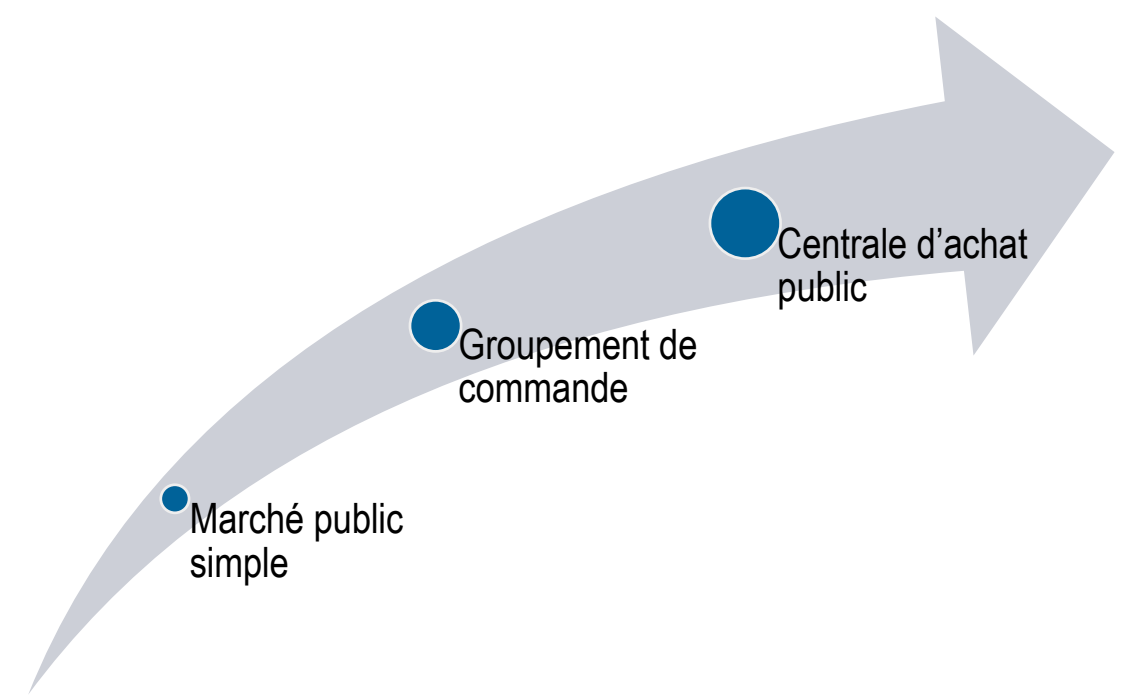

Note : Le groupement de commandes est une étape intermédiaire entre une centrale d'achat institutionnalisée et un marché publique simple

\section{Encourager les groupements de commande afin de réaliser des économies}

Afin de tirer tous les bénéfices de la création d'un groupement de commandes, il faut s'assurer de l'homogénéité des besoins entre les membres du groupement, mais également d'autres critères, tels que la situation financière et le respect du délai de paiement de chaque partenaire, des conditions de livraison harmonisées et un calendrier d'exécution du marché similaire (OCDE, 2018[6]).

En Algérie, le système de passation des marchés publics est décentralisé, toutefois le DMP prévoit un instrument de centralisation : le groupement de commandes. D'après l'article 36 du DMP, le groupement est formé par convention entre plusieurs services contractants, qui peuvent désigner parmi eux un service contractant coordinateur en charge de la passation du marché. Les modalités d'application de cet article sont précisées par arrêté du ministre des Finances, mais le seul arrêté disponible date du 28 mars 2011 (Ministère des Finances, Algérie, 2011 [47]]). D'après les missions exploratoires de l'OCDE, l'utilisation par les entités publiques des groupements de commandes est limitée. Par exemple, elle est utilisée pour mutualiser les acquisitions d'autobus pour le transport scolaire entre plusieurs municipalités.

La grande décentralisation de son système de marchés publics suggère que l'Algérie gagnerait à encourager la constitution de groupements de commandes entre différents services contractants dont les besoins sont homogènes. Comme détaillé dans la section 4.2, l'harmonisation des processus de programmation achat et la consolidation des plans d'achats de chaque service contractant via le futur système de passation électronique des marchés publics pourraient faciliter la constitution de groupements de commandes. 


\section{Pour la création de centrales d'achats publics généralistes}

La centralisation des achats agrège généralement les besoins d'un plus grand nombre de services contractants. La majorité des pays de l'OCDE ont mis en place une ou plusieurs centrales d'achats publics, et près d'un tiers d'entre eux ont des centrales d'achats à la fois au niveau central et au niveau régional (graphique 4.5). Les centrales d'achats peuvent également être sectorielles et spécialisées (santé, défense nationale, ou encore éducation). L'efficacité de la centralisation dépend des instruments et des techniques utilisées. Par exemple, dans les pays de l'OCDE, les centrales d'achats agissent généralement comme gestionnaires des accords-cadres au profit d'autres entités adjudicatrices. Par ailleurs, les principaux motifs de la création des centrales d'achats sont économiques : ils s'agit d'obtenir de prix plus compétitifs via un pouvoir de négociation renforcé auprès des fournisseurs et la réduction des coûts de transaction administratifs (OCDE, 2017 $[1])$.

\section{Graphique 4.5. L'existence de centrales d'achats dans les pays de l'OCDE}

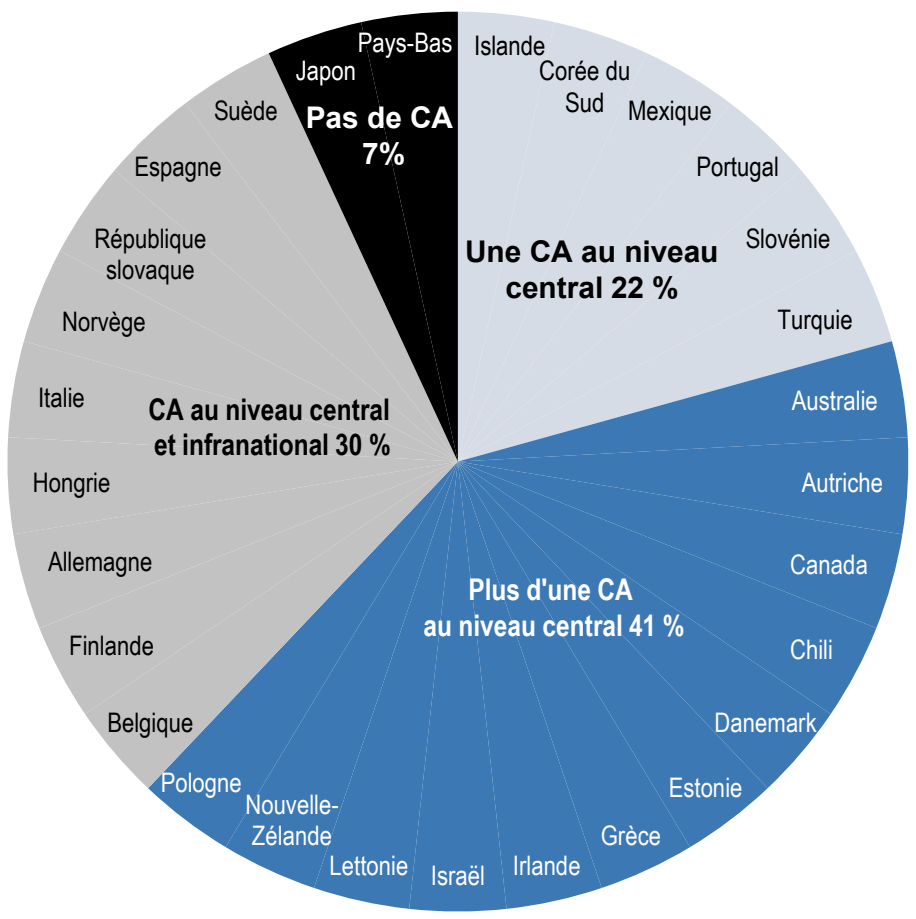

Note : 29 pays de l'OCDE.CA = centrale d'achats.

Source : (OCDE, 2016[43]).

Consciente des gains d'efficience obtenus par la centralisation, l'Algérie a mis en place trois centrales d'achats publics sectorielles, dont la plus importante est la Pharmacie centrale des hôpitaux, un établissement public industriel et commercial (EPIC) non soumis au DMP, sous l'égide du ministre de la Santé. Les marchés publics passés par les établissements publics de santé avec la Pharmacie centrale des hôpitaux ne sont pas non plus soumis au DMP. En revanche, il n'existe pas en Algérie de centrale d'achats généraliste ou interministérielle. Étant donné les gains d'efficience que peuvent générer les centrales d'achats, mais aussi l'homogénéité des besoins pour les achats dits " standards », l'Algérie pourrait envisager la création de centrales d'achat généralistes. Elles pourraient être mises en place au niveau central (interministériel), mais aussi au niveau des wilayas. 


\subsubsection{Promouvoir d'autres outils d'efficience dans les marchés publics}

\section{Améliorer le cadre réglementaire régissant les accords-cadres et promouvoir leur utilisation}

Un accord-cadre est un accord entre une entité adjudicatrice et un ou plusieurs opérateurs économiques pour la fourniture de biens et services et, plus rarement, de travaux, sur une période déterminée. II s'agit également d'un outil majeur de la mutualisation et de la centralisation des achats. Le but de l'accord-cadre est d'établir les termes du contrat à l'avance, notamment concernant les spécifications techniques minimales, et éventuellement un prix maximal (Mabin, 2018[8]). L'entité adjudicatrice passe ensuite les marchés subséquents par simple notification d'un marché d'application de l'accord-cadre au fournisseur sélectionné. Les accords-cadres offrent de nombreux avantages pour les entités adjudicatrices comme pour les fournisseurs, parmi lesquels une diminution de la lourdeur administrative, une compétition accrue, un meilleur accès des PME aux opportunités de marchés publics, un meilleur rapport qualité prix, une simplification des règles en les rendant plus flexibles, la promotion de l'innovation et une meilleure utilisation des ressources (OCDE, 2014[48]).

II existe quatre types d'accords-cadres en fonction du nombre de fournisseurs (un seul ou plusieurs) et de la définition ou non de tous les termes du contrat dans l'accord initial (accord complet ou incomplet) (tableau 4.3). L'accord-cadre mono-attribué correspond à un marché public "classique », attribué à un seul fournisseur, et où tous les termes du contrat sont déterminés.

\section{Tableau 4.3. Les quatre catégories d'accords-cadres}

\begin{tabular}{c|c|c|c}
\hline $\begin{array}{c}\text { Mono attribué } \\
\text { (un seul fournisseur) }\end{array}$ & $\begin{array}{c}\text { Multi attribué } \\
\text { (plusieurs fournisseurs) }\end{array}$ & $\begin{array}{c}\text { Mono attribué } \\
\text { (un seul fournisseur) }\end{array}$ & $\begin{array}{c}\text { Multi attribué } \\
\text { (plusieurs fournisseurs) }\end{array}$ \\
\hline Complet(termes définis) & Incomplet (termes indéfinis) & Incomplet (termes indéfinis) & Complet(termes définis) \\
\hline
\end{tabular}

Ce dispositif d'accords-cadres est adopté par les pays de l'OCDE, mais aussi par certains pays du MoyenOrient et d'Afrique du Nord (MENA). C'est le cas par exemple de l'Égypte qui a introduit en 2018 les accords-cadres dans son cadre réglementaire (loi $n^{\circ} 182$ sur les contrats des organismes publics). (Minsitère des Finances, Égypte, 2018[49]).

En Algérie, la réglementation prévoit les contrats-programmes (art. 33 du DMP) et les marchés à commande (art. 34 du DMP), qui peuvent porter sur une durée maximale de cinq années et « chevaucher sur deux ou plusieurs exercices budgétaires ». Les marchés à commande portent sur des prestations (fournitures, services ou travaux) courantes et à caractère répétitif. Ils doivent comporter l'indication de limites minimales et maximales (en termes de quantité et/ou en valeur) et ne sont conclus qu'avec un seul fournisseur (art. 34 du DMP).

En théorie, les contrats-programmes peuvent être attribués à plusieurs opérateurs économiques et prévoir une remise en concurrence lors de la notification des marchés subséquents (" marchés d'application » d'après l'article 33 du DMP). Cependant les services contractants doivent définir en avance les termes du contrat, notamment la nature et l'importance des prestations à réaliser, la localisation, le montant et l'échéancier de réalisation. Le système en place en Algérie ne permet donc pas de mettre en place des contrats-programmes lorsque tous les termes ne sont pas définis (accord-cadre incomplet dans le tableau 4.3), ce qui limite la flexibilité et donc l'efficience des contrats-programmes. En outre, comme évoqué au chapitre 1 (section 1.1), l'arrêté du ministère des Finances fixant les modalités d'application des contrats-programmes (art. 33 du DMP) n'a jamais été adopté. Le cadre réglementaire des contrats programme se limite donc à l'article 33 du DMP et manque par conséquent de clarté et de précision.

D'après les missions exploratoires de l'OCDE, le recours aux contrats-programmes est extrêmement limité en Algérie. Des contrats-programmes ont notamment été passés dans le cadre des marchés de construction de l'autoroute Est-Ouest (chaque tronçon faisant l'objet d'un marché d'application différent) 
ou concernant des équipements médicaux onéreux et complexes (scanners ou imagerie à résonnance magnétique). Par ailleurs, les services contractants n'ont pas recours aux contrats-programmes avec remise en concurrence, bien que cette possibilité leur soit ouverte par la réglementation. De plus, il n'existe pas de lignes directrices pour guider les services contractants quant aux bonnes pratiques en la matière.

Afin d'améliorer l'efficience du système, l'Algérie gagnerait à clarifier la réglementation existante afin d'y intégrer les différents types de contrats-programmes : mono ou multi attribués, complets et incomplets. Par ailleurs, l'Algérie devrait élaborer et diffuser des lignes directrices sur les contrats-programmes et organiser des formations à destination des entités publiques afin de renforcer leurs capacités sur les différentes possibilités offertes par cet outil d'efficience. L'amélioration du cadre réglementaire régissant les accords-cadres facilitera également les groupements de commandes et éventuellement la mise en place de centrales d'achat public.

La durée de vie d'un contrat-programme est décidée lors de sa conception par le service contractant. Ce dernier doit prendre en compte à la fois les avantages d'un contrat-programme de longue durée (réduction des coûts administratifs, simplification des procédures grâce aux marchés d'application) et les risques liés à l'atténuation de la concurrence entre fournisseurs et l'exclusion des nouveaux entrants pour toute sa durée de vie (OCDE, 2011[50]). Ainsi, dans la réglementation européenne, la durée de vie d'un accordcadre ne dépasse normalement pas quatre ans. Cependant, une période plus courte est généralement utilisée dans la pratique européenne, par exemple un accord-cadre de deux ans renouvelable pour un an à deux reprises (formule « 2+1+1») (OCDE, 2014[48]).

Par conséquent, l'Algérie pourrait envisager de réduire la durée maximale des contrats-programmes à quatre ans et encourager, dans les lignes directrices susmentionnées, les services contractants à faire usage de contrats-cadres d'une durée plus courte si possible. Un accord-cadre d'une durée de cinq ans risque en effet d'être préjudiciable à la concurrence en garantissant le marché à un fournisseur ou un groupe de fournisseurs sur une longue durée. En cas de besoin exceptionnel, la nécessité d'un accordcadre d'une durée supérieure à quatre ans devrait être dûment justifiée par le service contractant et faire l'objet d'une autorisation préalable de l'ARMP.

Les accords-cadres sont plus adaptés à certaines catégories d'achat. La détermination des catégories de produits et services qui doivent faire l'objet d'un accord-cadre est une étape essentielle. Le graphique 4.6 décrit la méthodologie à suivre pour définir les catégories d'achat pour lesquels l'utilisation d'accordscadres est souhaitable. 


\section{Graphique 4.6. Accords-cadres : les étapes de la définition des catégories d'achat}

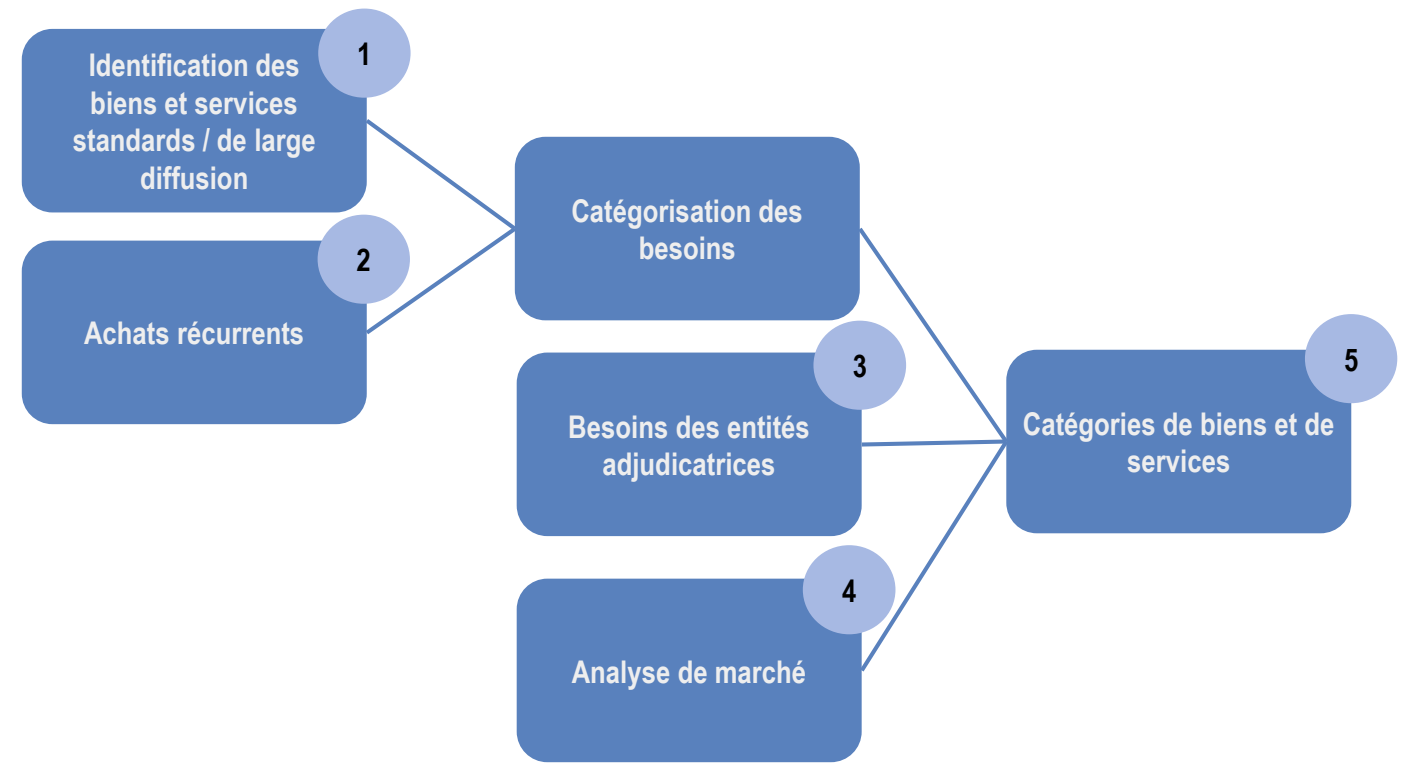

Source : (OCDE, 2014[48]).

À titre d'exemple, le tableau 4.4 détaille les catégories de biens et services qui font fréquemment l'objet d'un accord-cadre dans les pays de l'Union européenne (UE). Dans le cadre de sa nouvelle réglementation (loi $N^{\circ} 182$ ) des marchés publics, l'Égypte a également dressé une liste des biens et services les plus appropriés pour faire l'objet d'un accord-cadre sur la base de l'analyse de l'historique des achats. L'Algérie gagnerait aussi à fournir dans ses lignes directrices une orientation aux acheteurs publics sur les catégories pour lesquelles l'utilisation de contrats-programme est souhaitable.

\section{Tableau 4.4.Catégories de biens et services fréquemment couverts par les accords-cadres de l'UE}

\begin{tabular}{l|l}
\hline $\begin{array}{l}\text { - Ordinateurs PC de bureau et portables } \\
\text { - Imprimantes et photocopieuses }\end{array}$ & $\begin{array}{l}\text { - Services de télécommunication } \\
\text { (Téléphonie fixe, mobile et services associés) } \\
\text { - Plateformes électroniques de marchés publics }\end{array}$ \\
\hline - Équipement informatique & - Logiciels divers (bureautique, etc.) \\
\hline - Équipement de bureau & - Services de nettoyage et d'entretien des locaux \\
\hline - Carburant ; électricité & - Voyage et hospitalité, services de réservation \\
\hline - Véhicules et motocycles & - Assurance pour véhicules et motocycles \\
\hline - Fournitures de bureau & - Transport et services de mobilité (taxis, etc.) \\
\hline - Restauration collective & - Services de sécurité et de surveillance \\
\hline
\end{tabular}

Source : (OCDE, 2014[48]).

Pour améliorer l'efficience des procédures, les entités publiques peuvent également utiliser le système d'acquisition dynamique (SAD), une procédure entièrement électronique d'achat de fournitures, services ou travaux courants préalablement déterminés dans les documents d'appel d'offres par le pouvoir adjudicateur. Le SAD est uniquement adapté lorsque l'objet de l'achat est disponible sur le marché. La différence essentielle entre le SAD et l'accord-cadre est que les opérateurs économiques peuvent rejoindre le SAD à tout moment (OCDE/SIGMA, 2018[51]).

La concurrence induite par le SAD bénéficie au pouvoir adjudicateur car le SAD permet d'obtenir des offres économiquement avantageuses et en adéquation avec les évolutions technologiques du marché le cas échéant. Ce système favorise également la participation des PME car celles-ci disposent de plus de temps 
pour soumettre leur candidature, contrairement à une procédure qui fixe une durée limitée pour la soumission.

L'Algérie pourrait donc envisager d'intégrer le SAD dans son cadre réglementaire et d'adapter son système de passation électronique des marchés à cet outil afin d'en améliorer l'efficience.

\section{Promouvoir l'utilisation des catalogues électroniques et des enchères électroniques inversées}

Les outils électroniques peuvent jouer un rôle clé dans l'amélioration de l'efficience du système, que ce soit au niveau de gain achat (en termes de prix payé) ou en termes administratifs. Les catalogues électroniques et les enchères inversées font partie de ces outils (OCDE, 2015[3]). Lors d'un processus de sélection de fournisseurs, les entités adjudicatrices peuvent demander aux soumissionnaires de remettre leurs offres par le biais de catalogues électroniques. Une fois le ou les fournisseurs sélectionnés, elles disposeront de ces catalogues accessibles sur internet (directement ou par le biais d'une plateforme), sur la base desquels les commandes seront émises. Ces catalogues contiennent les produits (bien ou services) objets du marché signé et sont régulièrement mis à jour par les fournisseurs. Les catalogues électroniques présentent de nombreux avantage, comme par exemple renforcer l'accessibilité et la transparence du marché, réduire les coûts administratifs ou encore rationaliser et réviser les procédures d'achat (Commission Européenne, 2010[52]).

Les enchères électroniques inversées permettent à différents opérateurs économiques en concurrence de soumissionner puis de réviser leurs prix à la baisse en temps réel, de façon anonyme. Le service contractant fixe les règles des enchères, l'opérateur économique qui offre le prix le plus compétitif à l'issue de la procédure se voit attribuer le marché. Bien que l'objet de l'enchère soit généralement le prix, elle peut également porter sur d'autres éléments quantifiables liés aux caractéristiques des produits ou aux délais de livraison. Les enchères électroniques sont un instrument pertinent pour les procédures portant sur la fourniture de biens standardisés (OCDE, 2016[53]).

Étant donné leur contribution à l'amélioration de l'efficience du système de passation des marchés publics, les catalogues électroniques et les enchères électroniques inversées sont utilisés dans un grand nombre de pays. Par exemple, dans plus de la moitié (53\%) des pays de l'OCDE, certains pouvoirs adjudicateurs ont intégré ces instruments à leur portail électronique de marchés publics. Dans plus d'un tiers des pays de l'OCDE (36\%), le portail électronique des marchés publics du gouvernement central comprend un module dédié aux enchères électroniques inversées, et $33 \%$ des pays de l'OCDE ont également la possibilité d'intégrer des catalogues électroniques à leur portail (OCDE, 2016[20]).

En Algérie, le DMP permet l'utilisation de catalogues électroniques et d'enchères électroniques inversées dans le cadre du portail électronique des marchés publics (art. 206 du DMP). Conformément aux bonnes pratiques internationales, le DMP réserve ces deux outils aux acquisitions de fournitures et de prestations de services courants. Cependant, d'après les missions exploratoires de l'OCDE, ces deux outils ne sont pas utilisés. Bien qu'ils puissent être utilisés hors système de passation électronique des marchés publics, la mise en place d'un tel système faciliterait leur utilisation. En plus de promouvoir l'utilisation de ces deux outils, l'Algérie devra donc s'assurer que des modules d'enchères électroniques inversées et de catalogues électroniques seront prévus lors du développement du portail électronique (voir chapitre 3).

\subsection{Renforcer l'évaluation du système de passation des marchés publics}

Dans un contexte où les pays cherchent à améliorer l'efficacité des services publics et à optimiser leurs coûts sous la pression conjointe des contraintes budgétaires et des exigences croissantes des citoyens, il est essentiel de conduire une évaluation périodique en profondeur du système de passation des marchés publics afin de vérifier l'atteinte d'objectifs prédéfinis et de remédier aux éventuelles déficiences du système. La 
performance du système de passation des marchés publics peut être évaluée à trois niveaux différents : au niveau de chaque procédure, au niveau des services contractants, et au niveau national (graphique 4.7). Ces niveaux sont étroitement liés, la performance de chaque procédure ayant un impact sur la performance globale du système de passation des marchés publics au niveau national (OCDE, 2018[6]).

\section{Graphique 4.7. Les trois niveaux pour mesurer la performance du système de passation des marchés publics}

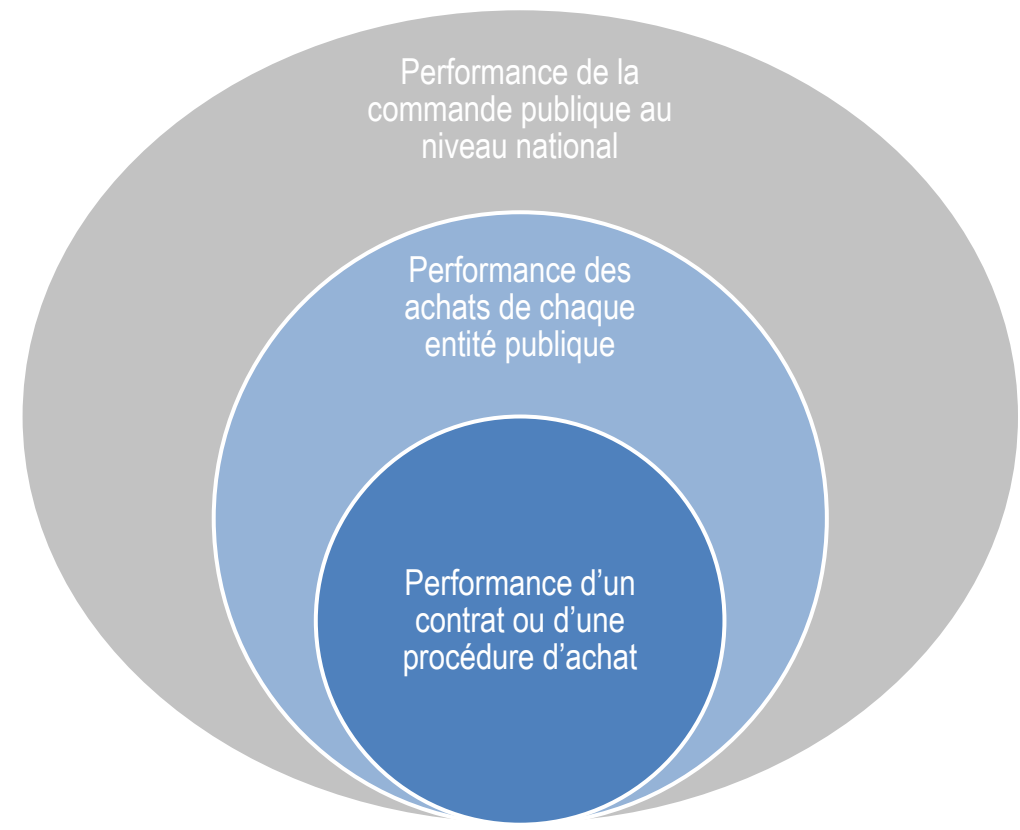

Source : Adapté de (OCDE, 2018[6]).

Selon la Recommandation du Conseil de l'OCDE sur les marchés publics, l'évaluation du système de passation des marchés publics requiert de :i) collecter des informations cohérentes, actualisées et fiables et utiliser les données relatives aux marchés antérieurs, notamment les prix et les coûts globaux; et ii) concevoir des indicateurs (indicateurs clés de performance) visant à mesurer la performance, l'efficacité et les économies liées au système de passation de marchés publics, à des fins de comparaison et afin d'éclairer la stratégie à suivre en matière de marchés publics (OCDE, 2015[3]). Toutefois, la collecte des données et la mesure des indicateurs clés de performance nécessitent la disponibilité de données pertinentes aux différents niveaux d'évaluation. Les systèmes d'information des marchés publics sont généralement une source majeure de données fiables et actualisées, qui peuvent être utilisées pour l'évaluation des marchés publics (OCDE, à paraître $\left.{ }_{[54]}\right)$.

\subsubsection{L'évaluation des marchés publics est en partie prévue dans les textes, mais pas encore appliquée}

\section{L'évaluation du système de passation des marchés publics nécessite un cadre institutionnel adapté et des progrès dans la dématérialisation des marchés publics}

Dans la plupart des pays de l'OCDE, une entité publique est chargée de l'évaluation globale de la performance du système de passation des marchés publics. Il s'agit généralement de l'organisme chargé des fonctions centrales (voir chapitre 1). Conformément aux bonnes pratiques internationales, cet organisme centralise les renseignements et les données de l'ensemble des pouvoirs adjudicateurs, de plus il développe des indicateurs de performance sur l'ensemble des procédures et produit des rapports d'évaluation permettant d'identifier les déficiences et axes d'amélioration du système de passation des 
marchés publics (OCDE, 2016[46] ; OCDE, 2013[55]). À titre d'exemple, en Pologne, l'agence des marchés publics centralise et publie les données et statistiques des marchés publics à l'échelle nationale. En Corée, l'agence chargée des marchés publics a des fonctions similaires, mais elle gère aussi le portail électronique de passation des marchés Koneps et dispose donc d'un accès direct aux données du portail, qui sont utilisées dans le cadre de sa mission d'évaluation des politiques et des réformes en matière de marchés publics (OCDE, 2016[20]).

En Algérie, l'article 213 du DMP confie à l'ARMP et à l'observatoire de la commande publique qui lui est rattaché, des missions de collecte et d'analyse de données, et d'évaluation de la commande publique. L'ARMP est notamment en charge d'effectuer un recensement économique annuel de la commande publique, d'analyser les données relatives aux aspects économiques et techniques de la commande publique et de faire des recommandations au gouvernement (art. 213 du DMP). En outre, dans le cadre du contrôle de tutelle (art. 164 du DMP), chaque service contractant établit un rapport d'évaluation portant sur les conditions de réalisation du projet d'achat et sur son coût global par rapport à l'objectif initial. Une copie de ce rapport est adressée à l'ARMP.

Cependant, l'ARMP n'a toujours pas été mise en place malgré l'entrée en vigueur du DMP en 2015. Sans la mise en place effective de l'ARMP, il n'est pas possible de réaliser une évaluation du système de passation des marchés publics. En effet, dans le cadre de cette revue, le ministère des Finances n'a pu transmettre à l'équipe OCDE aucune donnée chiffrée relative aux marchés publics, ce qui montre le manque d'études et d'évaluations chiffrées menées sur ce sujet. L'Algérie devrait donc mettre en place l'ARMP dans les plus brefs délais afin de mener des travaux relatifs à l'évaluation du système à différents niveaux, et de pouvoir produire des données chiffrées relatives au système de passation des marchés publics. En outre, en l'absence de portail électronique de passation des marchés publics, le ministère des Finances ne dispose pas de données fiables et actualisées relatives aux marchés publics. La mise en place de ce portail contribuerait à fournir les données nécessaires à l'évaluation de la passation des marchés publics.

\section{Pour des indicateurs de performance de la commande publique}

Dans les pays de l'OCDE, la disponibilité croissante de données agrégées de qualité permet de calculer des indicateurs clés de performance (Key performance indicators, KPI) afin de mesurer la performance du système de passation des marchés publics. Les indicateurs clés de performance sont utilisés pour mesurer l'atteinte d'objectifs conformes aux priorités stratégiques de chaque entité adjudicatrice ou du système de passation des marchés publics dans son ensemble (OCDE, 2018[19]). À cette fin, il est essentiel de choisir les indicateurs clés de performance adaptés et de s'assurer qu'ils remplissent les caractéristiques décrites dans l'encadré 4.8 . 


\section{Encadré 4.8. Développer des indicateurs clés de performance pertinents}

Les indicateurs clés de performance permettent d'évaluer le système de passation des marchés publics à différents niveaux, et donc d'adapter la stratégie d'achat. Tout indicateur clé de performance doit satisfaire un certain nombre de conditions afin de pouvoir être utilisé dans le cadre de la gestion stratégique des achats publics. Les indicateurs clés de performance doivent être :

- Pertinents : ils doivent être liés à des objectifs stratégiques plutôt que centrés sur des processus.

- Clairs : ils doivent être interprétés de façon identique par les différentes parties prenantes.

- Mesurables et objectifs : ils doivent être mesurés sur la base d'une formule ou d'une méthodologie prédéfinie et de données disponibles et objectives. Si ces données ne sont pas déjà disponibles, les coûts de collecte de ces données ne doivent pas être disproportionnés.

- Réalisables : les objectifs (indicateurs cibles) doivent être suffisamment réalistes.

- En nombre limité : les indicateurs doivent être limités en nombre tout en reflétant les objectifs stratégiques de l'organisation ou du système de passation des marchés publics.

- Limités dans le temps : le délai pour atteindre un indicateur clé de performance doit être clairement délimité.

Source : (OCDE, 2013[56]).

Les indicateurs doivent donc refléter les objectifs stratégiques de la passation des marchés publics. Par exemple, pour l'objectif d'accroître la concurrence afin d'obtenir un meilleur ratio qualité/prix, des indicateurs tels que le nombre moyen d'offres qualifiées par appel d'offres ou la part des procédures de gré à gré permettront de mesurer l'intensité de la concurrence et son évolution, au regard de l'objectif stratégique. Par ailleurs, les indicateurs clés de performance sont également nécessaire pour mesurer la contribution des marchés publics à des objectifs stratégiques de politique publique: sociaux, environnementaux ou en matière d'accès des PME à la commande publique. Par exemple, de nombreux pays de l'OCDE mesurent la part des marchés publics attribués à des PME et fixent des objectifs en la matière (OCDE, 2018[57]).

Compte tenu des pressions budgétaires que connaissent plusieurs pays, l'un des indicateurs de performance le plus répandu au sein des pays de l'OCDE mesure les économies réalisées ou " gains achats » lors de l'attribution d'un marché. II s'agit souvent de mesurer le prix suite à l'attribution du marché par rapport à un prix de référence. Selon les pays, le prix de référence peut être le dernier prix payé par l'entité publique lors du marché précédent, ou un prix déterminé grâce aux études de marché ou à un outil d'intelligence économique. Par ailleurs, de nombreux pays mesurent également l'efficacité au niveau des processus achat ou les gains de temps liés à l'introduction des systèmes électroniques de passation des marchés publics, ou à la mise en place d'un accord-cadre (OCDE, 2016[20]). L'encadré 4.9 décrit les indicateurs de performance de Colombia Compra Eficiente, l'agence en charge du système de passation des marchés publics en Colombie. 


\section{Encadré 4.9. Indicateurs clés de performance de Colombia Compra Eficiente}

L'agence Colombia Compra Eficiente a conçu onze indicateurs d'évaluation de la performance du système de passation des marchés publics colombien, mais aussi de chaque entité adjudicatrice. Sur la base des résultats des années précédentes, le gouvernement a mis en place en 2014 des valeurs cibles annuelles en liaison avec sa stratégie globale concernant les marchés publics. Le tableau cidessous présente six de ces indicateurs :

\begin{tabular}{l|l}
\hline \multicolumn{1}{c}{ Indicateur } & \multicolumn{1}{c}{ Objet } \\
\hline Délai moyen de passation du marché (nombre de jours) & $\begin{array}{l}\text { Période de temps entre le lancement de la procédure et la signature du } \\
\text { contrat }\end{array}$ \\
\hline Pourcentage de nouveaux fournisseurs & $\begin{array}{l}\text { Ratio des nouveaux fournisseurs de l'entité adjudicatrice (nouveaux } \\
\text { fournisseurs/total des fournisseurs de l'année précédente) }\end{array}$ \\
\hline Répartition du montant des achats par fournisseur & $\begin{array}{l}\text { Mesure à quel point le budget d'achats est concentré sur seulement quelques } \\
\text { fournisseurs (coefficient de Gini) }\end{array}$ \\
\hline $\begin{array}{l}\text { Pourcentage des marchés attribués à plusieurs fournisseurs } \\
\text { (accords-cadres) }\end{array}$ & $\begin{array}{l}\text { Fréquence des marchés attribués à plusieurs fournisseurs dans les accords- } \\
\text { cadres d'une entité adjudicatrice }\end{array}$ \\
\hline $\begin{array}{l}\text { Pourcentage des marchés attribués sans procédure } \\
\text { concurrentielle }\end{array}$ & $\begin{array}{l}\text { Part des marchés attribués via des processus sans appel à la concurrence } \\
\text { (gré à gré) }\end{array}$ \\
\hline Part des contrats faisant l'objet d'un avenant & $\begin{array}{l}\text { Proportion des contrats faisant l'objet d'un avenant (modification du contrat } \\
\text { après sa signature) }\end{array}$ \\
\hline
\end{tabular}

Source : (OCDE, 2016 $[58])$.

En Algérie, d'après les discussions menées avec les différentes parties prenantes, aucun indicateur n'a été développé pour mesurer la performance du système des marchés publics, y compris les indicateurs liés aux économies/gains d'achat. Bien que le développement d'indicateurs soit l'une des missions de l'ARMP, l'Algérie devrait réfléchir dès à présent au développement d'indicateurs visant à mesurer régulièrement la performance du système de passation des marchés publics, notamment les économies effectuées en lien avec les objectifs stratégiques nationaux.

\section{L'évaluation des fournisseurs gagnerait à une plus grande centralisation du fichier des opérateurs économiques}

La performance des fournisseurs est au cœur de la bonne exécution des marchés publics. Pour cette raison, la plupart des pays de l'OCDE disposent de mécanismes permettant d'exclure des candidats ou des soumissionnaires lorsque des défaillances importantes dans l'exécution des contrats ont été constatées. Par exemple, le ministère de la Fonction publique au Mexique peut exclure des opérateurs économiques de la participation aux marchés publics dans des cas prédéterminés, tels que la mauvaise foi dans l'exécution des obligations contractuelles ou la non-exécution du contrat aux torts exclusifs du fournisseur. La liste des opérateurs économiques ainsi sanctionnés est disponible en ligne (OCDE, $2016_{[20]}$.Pour les pays soumis à la Directive de l'UE de 2014, cette dernière prévoit que la performance passée d'un prestataire peut être prise en compte pour exclure un soumissionnaire d'une procédure d'appel d'offres pour autant que cette mauvaise performance ait été significative ou persistante (Union Européenne, 2014[7]).

L'Algérie a prévu dans son cadre réglementaire la mise en place de fichiers des opérateurs économiques dans lesquels chaque service contractant consigne les références professionnelles, les aptitudes et la qualification de ses fournisseurs. Ces informations doivent permettre « d'apprécier l'efficacité avec laquelle le fournisseur exécute ses engagements contractuels »(art. 58 du DMP). L'arrêté du ministre des 
Finances fixant le contenu et les conditions de mise à jour des fichiers des opérateurs économiques date de 2011, avant l'entrée en vigueur du décret régissant les marchés publics de 2015. L'arrêté de 2011 prévoit trois fichiers relatifs aux opérateurs économiques :

- un fichier au niveau de chaque service contractant comprenant des informations détaillées d'ordre technique, commercial, financier et sur la qualité de la relation commerciale établie avec le service contractant ;

- un fichier sectoriel concernant les « opérateurs économiques qui entretiennent des relations commerciales avec plusieurs services contractants du secteur »;

- un fichier national « dont les informations doivent permettre à l'observatoire économique de la commande publique d'exercer ses attributions en matière d'information des services contractants et en matière d'orientation des commandes publiques $"$.

Par ailleurs, comme mentionné au chapitre 2, le cadre réglementaire prévoit l'exclusion définitive ou temporaire de fournisseurs dans certains cas, notamment en cas de défaillance après avoir fait l'objet de décisions de résiliation aux torts exclusifs de leurs marchés par des services contractants. D'après l'arrêté du 19 décembre 2015 fixant les modalités d'inscription et de retrait de la liste des opérateurs économiques interdits de participer aux marchés publics, la liste est notifiée à l'ensemble des services contractants ou affichée sur le portail électronique des marchés publics. Les discussions menées lors des missions exploratoires de l'OCDE ont confirmé que les services contractants tentent de s'assurer, dans la pratique, que les marchés ne sont pas attribués à des entreprises figurant sur la liste des entreprises défaillantes dans leur secteur et qu'un fichier existe au niveau de chaque service contractant et sectoriel. Cependant, les services contractants n'ont aucune garantie quant à la mise à jour du fichier sectoriel. Par ailleurs, aucun fichier national n'a été développé. Sans la mise en place du portail électronique il est difficile de diffuser une liste actualisée et centralisée à tous les services contractants. En attendant la mise en place effective du portail, le ministère des Finances pourrait publier sur son site internet la liste des entreprises défaillantes et s'assurer de sa mise à jour, En effet cette mission est conforme aux prérogatives de la Division des marchés publics. De plus, l'Algérie devrait s'assurer de la mise en place du fichier national afin d'obtenir une base de donnée des opérateurs économiques au niveau national. La disponibilité de ce fichier sera également facilitée par la mise en place du portail électronique des marchés publics.

\subsubsection{Deux préalables à la mise en place d'indicateurs de performance : un système électronique de passation des marchés publics et une nomenclature unifiée}

\section{Vers une collecte de données via un système d'information électronique}

Afin de recueillir des informations cohérentes, actualisées et fiables sur le système de passation des marchés publics, l'utilisation de portails électroniques des marchés publics est indispensable. En effet, la transmission manuelle des données est inefficiente. L'expérience de certains pays, tels que l'Allemagne, montre que la transmission manuelle de fiches statistiques par les services contractants (y compris par courrier électronique) est insatisfaisante et entraîne des problèmes de qualité des données (interprétations divergentes des catégories statistiques) et de données manquantes (retards ou omissions). Cette expérience est l'une des raisons de réformes dans plusieurs pays, y compris en Allemagne, qui prévoient une transition vers un système d'échange automatisé de données depuis les systèmes électroniques des services contractants (OCDE, à paraître $\left.{ }_{[59]}\right)$. Dès lors, dans la plupart des pays de l'OCDE, les portails électroniques des marchés publics permettent d'alimenter une base de données via un échange automatique de donnée. Cette collecte automatisée permet de fournir des données fiables qui peuvent être utilisées pour l'évaluation des nouveaux besoins et le pilotage stratégique du système de passation des marchés publics. Ces données permettent de mesurer des indicateurs clés du système des marchés publics, afin de comparer la performance des services contractants entre eux et de détecter des irrégularités, des anomalies ou des cas de fraude (OCDE, 2016 $[25]$ ). 
En Algérie, l'article 214 du DMP prévoit l'établissement de fiches statistiques par les services contractants et leur transmission à l'ARMP. C'est un premier pas vers la collecte systématique de données concernant les marchés publics de l'ensemble du pays. Cependant, l'expérience de certains pays comme l'Allemagne suggère que les risques d'erreurs ou de données manquantes dans le cadre d'un tel système sont considérables. Dès lors, l'Algérie gagnerait à mettre en place un système national de passation électronique des marchés publics qui permettrait la collecte automatisée et systématique des données nécessaires à l'évaluation de la commande publique (recensement économique de la commande publique).

\section{Pour la mise en place d'une nomenclature d'achat unifiée pour toute l'Algérie}

Une nomenclature codifiée et unique permet de standardiser les références utilisées par les services contractants pour décrire l'objet d'un marché. Dans une nomenclature, un code unique est associé à un produit ou un service. L'utilisation de ces nomenclatures permet donc aux opérateurs économiques d'identifier plus rapidement les opportunités d'achat dans leur secteur d'activité. Elle est indispensable au recensement économique de la commande publique, car elle facilite grandement l'analyse de données agrégées sur les marchés publics dans l'ensemble d'un pays. À titre d'exemple, dans les pays de l'UE, le code CPV (Common Procurement Vocabulary, ou vocabulaire commun pour les marchés publics) est d'utilisation obligatoire depuis le 1er février 2006 (encadré 4.10) (SIMAP, 2008[60]).

\section{Encadré 4.10. La nomenclature de l'UE de classification des marchés publics CPV}

Le code CPV est le système de classification (ou nomenclature) pour les marchés publics dans I'UE. II associe à chaque code numérique la description d'un objet de marché. II permet aussi aux entreprises de repérer facilement les appels d'offres qui les concernent.

Le code CPV est utilisé pour tous les appels d'offres publiés dans le supplément au Journal officiel de I'UE (plateforme Tenders Electronic Daily). II repose sur une structure arborescente de codes comptant jusqu'à neuf chiffres auxquels correspond un intitulé qui décrit les fournitures, travaux ou services objets du marché. Au total, le vocabulaire principal comprend 9454 codes. Les codes CPV en vigueur datent de 2008 ; les services de la Commission européenne en préparent actuellement une version mise à jour.

Des données d'enquêtes de 2012 montrent que le code CPV est jugé utile par les pouvoirs adjudicateurs et les opérateurs économiques. Notamment, $57 \%$ des soumissionnaires ont répondu que ces codes leur permettaient de repérer un plus grand nombre d'avis pertinents.

Sources : (SIMAP, 2008[60]) ; (Commission Européenne, 2008[61]) ; (Union Européenne, 2014[7]) ; (BME \& European Commission, 2012[62])

Il existe également d'autres systèmes de classification dans le domaine des marchés publics, notamment la nomenclature UNSPSC (United Nations Standard Products and Services Code, ou code de classification des produits et services des Nations Unies) développée sous l'égide du Programme des Nations Unies pour le développement (PNUD). II s'agit d'une nomenclature à huit chiffres pour classifier à la fois les produits et les services, qui comprend 87477 catégories distinctes de produits et services (UNSPSC, 2017[63]). L'UNSPSC est utilisée par les Nations Unies pour ses achats (portail d'achats commun du système des organismes des Nations Unies) et par certains pays pour leurs marchés publics, comme par exemple le Canada (Services publics et Approvisionnement Canada (SPAC), 2018[64]).

En parallèle de la mise en place du système d'information électronique des marchés publics, l'Algérie devrait mettre en place un système de nomenclature codifiée et unique qui permettrait, d'une part, d'améliorer l'accès des fournisseurs aux opportunités de marchés publics et, d'autre part, de collecter des 
données cohérentes et fiables concernant différentes catégories d'achat. Une telle nomenclature unifiée devrait être intégrée au système d'information des marchés publics.

Deux options sont possibles pour l'Algérie : la mise en place de sa propre nomenclature inspirée des grandes nomenclatures mondiales, ou l'adoption d'une nomenclature existante. Dans ce dernier cas, il serait souhaitable d'opter pour la version actualisée de la nomenclature européenne qui devrait être disponible prochainement. 


\section{Propositions d'action}

\begin{tabular}{|c|c|c|c|}
\hline Recommandations & $\begin{array}{l}\text { Court } \\
\text { terme }\end{array}$ & $\begin{array}{c}\text { Moyen } \\
\text { terme }\end{array}$ & $\begin{array}{l}\text { Long } \\
\text { terme }\end{array}$ \\
\hline Renforcer les capacités des services contractants en matière d'analyse des besoins & & $\mathrm{X}$ & \\
\hline $\begin{array}{l}\text { Veiller au respect de l'obligation de publication des programmes prévisionnels au BOMOP et/ou sur leur site } \\
\text { internet par les services contractants }\end{array}$ & $\mathrm{X}$ & & \\
\hline Développer un format standard pour les programmes prévisionnels des projets de marché & $X$ & & \\
\hline Formaliser le processus de consultation citoyenne au niveau des communes & & $\mathrm{X}$ & \\
\hline $\begin{array}{l}\text { Renforcer les études d'identification et les études de faisabilité des investissements dans la phase amont de } \\
\text { la maturation des projets }\end{array}$ & $X$ & & \\
\hline $\begin{array}{l}\text { Utiliser les consultations citoyennes dans l'identification et la priorisation des besoins au niveau de chaque } \\
\text { Wilaya, dans le cadre de l'élaboration des plans sectoriels déconcentrés (PSD) }\end{array}$ & & $\mathrm{X}$ & \\
\hline $\begin{array}{l}\text { Inciter les services en charge de la rédaction des documents d'appel d'offres à améliorer la coordination avec } \\
\text { les services requérants }\end{array}$ & & $X$ & \\
\hline Renforcer les études de marché conduites par les services contractants & & $\mathrm{X}$ & \\
\hline Renforcer les capacités des acheteurs publics en matière d'études de marché & & $\mathrm{X}$ & \\
\hline Élaborer des lignes directrices ou un guide pratique en matière d'études de marché & $X$ & & \\
\hline $\begin{array}{l}\text { Élaborer des guides en matière d'études de marchés spécifiques par secteur (travaux publics, technologies de } \\
\text { l'information, etc.) }\end{array}$ & & $\mathrm{X}$ & \\
\hline $\begin{array}{l}\text { Mettre en place une bibliothèque d'études de marché à disposition des services contractants sur portail } \\
\text { électronique des marchés publics }\end{array}$ & & & $\mathrm{X}$ \\
\hline $\begin{array}{l}\text { Améliorer l'analyse du marché en mettant en place des dialogues formalisés entre les services contractants et } \\
\text { le secteur privé (y compris demande d'information et rencontres avec les fournisseurs) }\end{array}$ & & $\mathrm{X}$ & \\
\hline $\begin{array}{l}\text { Utiliser le système de passation électronique des marchés publics dans le cadre des consultations avec les } \\
\text { fournisseurs pour diffuser des demandes d'information, annoncer les rencontres avec les fournisseurs, et } \\
\text { publier leurs comptes rendus }\end{array}$ & & & $X$ \\
\hline $\begin{array}{l}\text { Renforcer les prérogatives de la CNED et rendre son avis favorable obligatoire avant le lancement d'un projet } \\
\text { d'infrastructure économique et sociale }\end{array}$ & & $\mathrm{X}$ & \\
\hline Doter la CNED du statut d'organisme indépendant & & $\mathrm{X}$ & \\
\hline $\begin{array}{l}\text { Élargir le périmètre d'intervention de la CNED, renforcer les études complémentaires qu'elle mène et conduire } \\
\text { davantage d'études complémentaires }\end{array}$ & & $\mathrm{X}$ & \\
\hline $\begin{array}{l}\text { Développer des lignes directrices s'agissant des critères de qualification pour éviter qu'ils ne soient } \\
\text { excessivement restrictifs. }\end{array}$ & & $X$ & \\
\hline $\begin{array}{l}\text { Encourager les services contractants à utiliser davantage la méthode du ratio prix/efficacité (ou rapport } \\
\text { qualité/prix) }\end{array}$ & $\mathrm{X}$ & & \\
\hline Mettre en place l'ARMP & $\mathrm{X}$ & & \\
\hline $\begin{array}{l}\text { Développer un guide ou des lignes directrices à destination des services contractants concernant l'utilisation } \\
\text { de différents critères de sélection. }\end{array}$ & & $\mathrm{X}$ & \\
\hline Renforcer les capacités des acheteurs publics concernant l'utilisation des critères de sélection & & $\mathrm{X}$ & \\
\hline $\begin{array}{l}\text { Développer des lignes directrices pour sensibiliser les entités publiques au concept du coût global } \\
\text { d'acquisition et mener des actions de formation à ce sujet }\end{array}$ & & $\mathrm{X}$ & \\
\hline $\begin{array}{l}\text { Publier gratuitement des indices suffisamment détaillés, pertinents et à jour pour être utilisés dans les clauses } \\
\text { de révision des prix des contrats publics }\end{array}$ & $\mathrm{X}$ & & \\
\hline $\begin{array}{l}\text { Confier à l'Office National des Statistiques (ONS) la centralisation et la publication sur son site de l'ensemble } \\
\text { des indices de référence pour les formules de révision des prix utilisées dans les marchés publics et s'assurer } \\
\text { de la coopération entre les Ministères sectoriels concernés, y compris celui de l'Habitat, de l'Urbanisme et de } \\
\text { la Ville, et l'ONS }\end{array}$ & & & $\mathrm{X}$ \\
\hline $\begin{array}{l}\text { Encourager la constitution de groupements de commandes entre services contractants dont les besoins sont } \\
\text { homogènes. }\end{array}$ & & $\mathrm{X}$ & \\
\hline Envisager la création de centrale(s) d'achats généraliste(s) & & & $\mathrm{X}$ \\
\hline Clarifier la règlementation existante afin d'y intégrer les différents types de contrats-programmes & $\mathrm{X}$ & & \\
\hline Élaborer et diffuser des lignes directrices sur les contrats-programmes & & $\mathrm{X}$ & \\
\hline $\begin{array}{l}\text { Fournir dans ces lignes directrices une orientation aux acheteurs publics sur les catégories pour lesquelles } \\
\text { l'utilisation de contrats-programme est souhaitable }\end{array}$ & & $\mathrm{X}$ & \\
\hline Renforcer les capacités en terme d'utilisation des outils d'efficience & & $\mathrm{X}$ & \\
\hline Améliorer le cadre réglementaire régissant les accords-cadres afin de faciliter les groupements de & & & $X$ \\
\hline
\end{tabular}




\begin{tabular}{|c|c|c|c|}
\hline commandes et éventuellement la mise en place de centrales d'achat public. & & & \\
\hline Réduire la durée maximale des contrats-programmes à quatre ans & & & $\mathrm{X}$ \\
\hline $\begin{array}{l}\text { Fournir des lignes directrices ou une orientation aux acheteurs publics sur les catégories pour lesquelles } \\
\text { l'utilisation de contrats-programme est souhaitable }\end{array}$ & & $\mathrm{X}$ & \\
\hline $\begin{array}{l}\text { Intégrer le système d'acquisition dynamique (SAD) dans le cadre réglementaire et l'intégrer à son système de } \\
\text { passation électronique des marchés }\end{array}$ & & & $\mathrm{X}$ \\
\hline $\begin{array}{l}\text { S'assurer que des modules d'enchères électroniques inversées et de catalogues électroniques seront prévus } \\
\text { lors du développement du portail électronique }\end{array}$ & & $\mathrm{X}$ & \\
\hline $\begin{array}{l}\text { Mener des travaux relatifs à l'évaluation du système à différents niveaux et produire des données chiffrées } \\
\text { relatives au système de passation des marchés publics (grâce à la mise en place de l'ARMP) }\end{array}$ & & & $\mathrm{X}$ \\
\hline $\begin{array}{l}\text { Développer des indicateurs visant à mesurer régulièrement la performance du système de passation des } \\
\text { marchés publics, y compris les économies effectuées }\end{array}$ & $\mathrm{X}$ & & \\
\hline $\begin{array}{l}\text { Obtenir les données nécessaires à l'évaluation de la passation des marchés publics grâce à la mise en place } \\
\text { du portail électronique de passation des marchés publics }\end{array}$ & & & $\mathrm{X}$ \\
\hline $\begin{array}{l}\text { Publier sur le site internet du ministère des Finances la liste des entreprises défaillantes et assurer sa mise à } \\
\text { jour }\end{array}$ & & $\mathrm{X}$ & \\
\hline $\begin{array}{l}\text { Assurer la mise en place du fichier national des entreprises défaillantes afin d'obtenir une base de donnée des } \\
\text { opérateurs économiques au niveau national }\end{array}$ & & $\mathrm{X}$ & \\
\hline $\begin{array}{l}\text { Assurer la collecte automatisée et systématique des données nécessaires à l'évaluation de la commande } \\
\text { publique grâce à au portail électronique de passation des marchés publics }\end{array}$ & & & $\mathrm{X}$ \\
\hline $\begin{array}{l}\text { Mettre en place un système de nomenclature d'achats codifiée et unique intégrée au portail électronique de } \\
\text { passation des marchés publics }\end{array}$ & & $\mathrm{X}$ & \\
\hline
\end{tabular}

Note : Court terme : recommandation à mettre en application dans les 12 mois ;

Moyen terme : recommandation à mettre en application dans les 1 à 2 ans ;

Long terme : recommandation à mettre en application dans plus de 2 ans. 


\section{Références}

Banque Mondiale (2018), Algérie : rapport de suivi de la situation économique, http://www.banquemondiale.org/fr/country/algeria/publication/economic-outlook-october-2018 (consulté le 8 février 2019).

Banque Mondiale (2007), Une Revue des dépenses publiques, https://siteresources.worldbank.org/INTALGERIAINFRENCH/Resources/ALGERIA.PER.Fren ch.Volumel.pdf (consulté le 11 février 2019).

BME \& European Commission (2012), Review of the Functioning of the CPV Codes/System, https://ec.europa.eu/docsroom/documents/21583/attachments/1/translations/en/renditions/nat ive.

Caisse nationale d'équipement pour le développement (CNED) (2008), Guide de maturation des grands projets d'infrastructure économique et sociale.

Choi, H. (2009), « Participatory Budgeting in Buk-gu, Korea », dans Focus on Citizens : Public Engagement for Better Policy and Services, OECD Publishing, Paris, https://dx.doi.org/10.1787/9789264048874-15-en.

Commission Européenne (2010), Développement des marchés électroniques dans l'Union européenne (Livre vert), https://eur-lex.europa.eu/legalcontent/FR/TXT/HTML/?uri=LEGISSUM:mi0060\&from=FR (consulté le 8 février 2019).

Commission Européenne (2008), European Code of Best Practices Facilitating Access by SMEs to Public Procurement Contracts, http://dx.doi.org/www.ecec.net/fileadmin/pdf/law/2/smecodeofbestpracticesen1.pdf.

Commission Européenne (2008), Guide du vocabulaire common pour les marchés publics (CPV), https://simap.ted.europa.eu/documents/10184/36234/cpv 2008 guide fr.pdf.

Crown Agents (2018), Guidelines on public procurement award criteria: best price quality ratio, http://eupublicprocurement.org.ua/wp-content/uploads/2017/01/PPL- (consulté le 6 mars 2019).

EBRD (2015), Are you ready for eProcurement? Guide to Electronic Procurement Reform, https://www.ppi-ebrduncitral.com/images/stories/pdf/151208 Are you ready for eProcurement.pdf (consulté le 13 décembre 2018).

Fonds Monétaire International (2018), World Economic Database, https://www.imf.org/external/pubs/ft/weo/2018/02/weodata/index.aspx (consulté le 5 juillet 2019).

Gouvernment d'Australie (2019), Annual Procurement Plan View, https://www.tenders.gov.au/?event=public.app.view\&appuuid=88596DD3-9E9C-801EDF8925457F8FB096 (consulté le 11 février 2019).

INE (s.d.), INEbase / Industria, energía y construcción /Construcción y vivienda /índices de precios de materiales e índices nacionales de la mano de obra / Resultados, https://www.ine.es/dyngs/INEbase/es/operacion.htm?c=Estadistica C\&cid=1254736154972\& menu=resultados\&idp=1254735576757 (consulté le 31 janvier 2019). 
JORA (2004), Journal officiel de la République Algérienne du 6 Juin 2004 (17 Rabie Ethani 1425), http://www.cned.dz/images/DECRET\%20CNED.pdf.

Le Moniteur (s.d.), Le prix dans les marchés publics Guide et recommandations, https://www.lemoniteur.fr/article/le-prix-dans-les-marches-publics-guide-etrecommandations.1138869 (consulté le 11 février 2019).

Mabin, D. (2018), Marchés publics: notions, procédures, voies de recours, Studyrama, https://www.lgdi.fr/marches-publics-9782759036912.html.

Ministère de l'Économie et des Finances (DAJ) (2013), Le prix dans les marchés publics, https://www.economie.gouv.fr/files/files/directions services/daj/marches publics/oeap/concert ation/autres groupes travail/guide-prix-dans-mp.pdf (consulté le 8 février 2019).

Ministère de l'Economie et des Finances, France (2019), DAJ - OECP - Liste des guides Groupes d'experts des marchés, https://www.economie.gouv.fr/daj/oecp-liste-des-guidesgem (consulté le 5 mars 2019).

Ministère de l'Intérieur, Algérie (2015), Fiche pédagogique relative au Plan communal de développement.

Ministère des Finances, Algérie (2014), Baisse du recours aux réévaluations des grands projets d'équipement public, http://www.mf.gov.dz/article/2/A-la-Une/453/CNED:-Baisse-du-recoursaux-r\%C3\%A9\%C3\%A9valuations-des-grands-projets-d\%E2\%80\%99\%C3\%A9quipementpublic.html (consulté le 5 juillet 2019).

Ministère des Finances, Algérie (2011), Arrêté du 28 mars 2011 fixe les modalités de constitution et de fonctionnement des groupements de commandes, http://www.andi.dz/PDF/legislation/autre/24-2011-8.pdf.

Ministry of Business, Innovation and Employment (2011), Mastering procurement: a structured approach to strategic procurement, https://www.procurement.govt.nz/assets/procurementproperty/documents/guide-mastering-procurement.pdf.

Minsitère des Finances, Égypte (2018), Government contracting law modernisation: centralised procurement approach and experience (unpublished).

New Zealand Government Procurement Branch (2015), Government Rules of Sourcing, https://www.procurement.govt.nz/assets/procurement-property/documents/government-rulesof-sourcing-procurement.pdf (consulté le 14 août 2018).

OCDE (2019), Inflation (CPI) (indicateur), https://dx.doi.org/10.1787/eee82e6e-en (consulté le 5 août 2019).

OCDE (2018), 2018 OECD Survey on the Implementation of the 2015 OECD Recommendations on Public Procurement.

OCDE (2018), Procurement Review of the Chamber of Commerce of Bogotá, Colombia: Aligning Practices with the OECD Public Procurement Recommendation, OCDE, Paris, https://www.oecd.org/gov/public-procurement/publications/procurement-review-chambercommerce-bogota.pdf. 
OCDE (2018), Second Public Procurement Review of the Mexican Institute of Social Security (IMSS) : Reshaping Strategies for Better Healthcare, Examens de l'OCDE sur la gouvernance publique, Éditions OCDE, Paris, https://dx.doi.org/10.1787/9789264190191-en.

OCDE (2018), SMEs in Public Procurement : Practices and Strategies for Shared Benefits, Examens de l'OCDE sur la gouvernance publique, Éditions OCDE, Paris, https://dx.doi.org/10.1787/9789264307476-en.

OCDE (2017), Getting Infrastructure Right: A framework for better governance, Éditions OCDE, Paris, https://dx.doi.org/10.1787/9789264272453-en.

OCDE (2017), Panorama des administrations publiques 2017, Éditions OCDE, Paris, http://dx.doi.org/10.1787/gov glance-2017-fr.

OCDE (2017), Participatory democracy: Portugal's new frontier, http://www.oecd.org/governance/participatory-democracy-portugal-new-frontier.htm (consulté le 30 janvier 2019).

OCDE (2017), Public Procurement in Chile: Policy Options for Efficient and Inclusive Framework Agreements, Examens de l'OCDE sur la gouvernance publique, Éditions OCDE, Paris, https://dx.doi.org/10.1787/9789264275188-en.

OCDE (2016), 2016 OECD Survey on Public Procurement, OCDE, Paris, http://www.oecd.org/gov/2016-OECD-Survey-on-Public-Procurement.pdf.

OCDE (2016), Checklist for Supporting the Implementation of the OECD Recommendation of the [46] Council on Public Procurement, OCDE, Paris, http://www.oecd.org/governance/procurement/toolbox/search/checklist-implementation-oecdrecommendation.pdf (consulté le 31 janvier 2019).

OCDE (2016), Improving ISSSTE's Public Procurement for Better Results, Examens de l'OCDE sur la gouvernance publique, Éditions OCDE, Paris, http://dx.doi.org/10.1787/9789264249899-en.

OCDE (2016), Panorama des administrations publiques 2015, Éditions OCDE, Paris, https://dx.doi.org/10.1787/gov glance-2015-fr.

OCDE (2016), « Réponses au questionnaire de l'OCDE sur les marchés publics ».

OCDE (2016), The Korean Public Procurement Service: Innovating for Effectiveness, Examens de l'OCDE sur la gouvernance publique, Éditions OCDE, Paris, https://dx.doi.org/10.1787/9789264249431-en.

OCDE (2016), Towards Efficient Public Procurement in Colombia: Making the Difference, Examens de l'OCDE sur la gouvernance publique, Éditions OCDE, Paris, https://dx.doi.org/10.1787/9789264252103-en.

OCDE (2015), Recommandation du Conseil de l'OCDE sur les marchés publics, OCDE, Paris, http://www.oecd.org/fr/gov/ethique/Recommandation-OCDE-sur-les-marches-publics.pdf (consulté le 16 août 2018).

OCDE (2014), Fighting Bid Rigging in Public Procurement in Colombia, OCDE, Paris, https://www.oecd.org/daf/competition/Booklet SIC\%20Procurement\%20Report 16X23 REV web.pdf (consulté le 5 décembre 2018). 
OCDE (2014), Manual for Framework Agreements, OCDE, Paris, https://www.oecd.org/gov/ethics/manual-framework-agreements.pdf (consulté le 17 décembre 2018).

OCDE (2013), « Organising Central Public Procurement Functions », SIGMA Public Procurement Briefs, $n^{\circ} 26$, Éditions OCDE, Paris, https://dx.doi.org/10.1787/5js4vmn5s5kden.

OCDE (2013), Public Procurement Review of the Mexican Institute of Social Security : Enhancing Efficiency and Integrity for Better Health Care, Examens de l'OCDE sur la gouvernance publique, Éditions OCDE, Paris, https://dx.doi.org/10.1787/9789264197480en.

OCDE (2013), Public Procurement Review of the State's Employees' Social Security and Social Services Institute in Mexico, Examens de l'OCDE sur la gouvernance publique, Éditions OCDE, Paris, https://dx.doi.org/10.1787/9789264197305-en.

OCDE (2012), Recommandation du conseil de l'OCDE sur la lutte contre les soumissions concertées dans les marchés publics, OCDE, Paris, http://www.oecd.org (consulté le 19 juillet 2018).

OCDE (2011), "Centralised Purchasing Systems in the European Union », SIGMA Papers, $\mathrm{n}^{\circ}$ 47, Éditions OCDE, Paris, https://dx.doi.org/10.1787/5kgkgqv703xw-en.

OCDE (2011), «Framework Agreements », SIGMA Public Procurement Briefs, n 19, Éditions OCDE, Paris, https://dx.doi.org/10.1787/5js4vmnmnhf7-en.

OCDE (2011), "Setting the Award Criteria », SIGMA Public Procurement Briefs, $\mathrm{n}^{\circ}$ 8, Éditions OCDE, Paris, https://dx.doi.org/10.1787/5js4wzvcz69q-en.

OCDE (à paraître), Enhancing the Use of Competitive Tendering in Costa Rica's Public Procurement System, OCDE, Paris.

OCDE (à paraître), Examen d'intégrité dans les marchés publics au Québec.

OCDE (à paraître), Public Procurement in Germany : Strategic Dimensions for Well-being and Growth, Examens de l'OCDE sur la gouvernance publique, Éditions OCDE, Paris, https://dx.doi.org/10.1787/1db30826-en.

OCDE (à paraître), Public Procurement Review of Kazakhstan.

OCDE/SIGMA (2018), Presentation, SIGMA Public Procurement Workshop, Dynamic Purchasing Systems, Turkey, 10 April 2018, https://www.slideshare.net/SIGMA2013/presentation-sigma-public-procurement-workshopdynamic-purchasing-systems-turkey-10-april-2018 (consulté le 16 mai 2019).

Sanchez-Graells, A. et I. Herrera Anchustegui (2016), « Impact of public procurement aggregation on competition. Risks, rationale and justification for the rules in Directive 2014/24 », pp. 129-163, http://dx.doi.org/10.2139/ssrn.2534496.

Scottish Government (2016), Procurement of care and support services : best practice guidance, https://www.gov.scot/publications/guidance-procurement-care-support-services-2016-bestpractice/pages/4/ (consulté le 5 juillet 2019). 
Services publics et Approvisionnement Canada (SPAC) (2018), Mise en œuvre de la classification UNSPSC, https://achatsetventes.gc.ca/donnees-sur-lapprovisionnement/unspsc (consulté le 23 janvier 2019).

SIGMA (2017), "Tender Evaluation and Contract Award », SIGMA Public Procurement Brief 9, http://www.sigmaweb.org/publications/Public-Procurement-Policy-Brief-9-200117.pdf (consulté le 31 janvier 2019).

SIGMA (2016), "Contract modifications », SIGMA Public Procurement Brief 38, http://www.sigmaweb.org/publications/Public-Procurement-Policy-Brief-38-200117.pdf (consulté le 8 février 2019).

SIMAP (2008), Système de classification unique CPV pour les marchés publics, https://simap.ted.europa.eu/fr/web/simap/cpv\# (consulté le 1 février 2019).

State of Queensland (Dpt of Housing and Public Works) (2018), Supply Market Analysis, http://www.hpw.qld.gov.au/SiteCollectionDocuments/ProcurementGuideSupplyMarketAnalysi s.pdf (consulté le 6 mars 2019).

Union Européenne (2014), Directive 2014/24/UE sur la passation des marchés publics, https:/leur-lex.europa.eu/legal-content/FR/TXT/PDF/?uri=CELEX:32014L0024\&from=SL (consulté le 8 mars 2019).

UNSPSC (2017), Newsletter UNSPSC (Sept. 2017), http://www.unspsc.org/library/september2017-newsletter (consulté le 23 janvier 2019). 


\section{La contribution des marchés à la} réalisation des objectifs des politiques publiques en Algérie

En Algérie, l'utilisation des marchés publics comme levier stratégique pour atteindre des objectifs de politiques publiques est relativement limitée malgré quelques dispositions prévues dans le cadre réglementaire. Ce chapitre propose un état des lieux du cadre réglementaire et évalue sa cohérence avec les politiques nationales et les pratiques des entités publiques. II fournit des recommandations pour une meilleure prise en compte des politiques publiques dans les marchés publics, notamment à travers le développement et l'implémentation d'une stratégie dans ce domaine. 
De nombreux gouvernements utilisent de plus en plus les marchés publics comme un outil stratégique pour contribuer à la réalisation d'objectifs de politiques publiques (OPP) nationales. En effet, l'objectif principal de la passation du marché est la fourniture des biens et services nécessaires à l'exécution de la mission des pouvoirs publics en temps opportun, au moindre coût et avec efficience. Les objectifs complémentaires quant à eux regroupent divers objectifs tels que le développement durable, la préservation de l'environnement, développement de petites et moyennes entreprises (PME), l'innovation, l'instauration de normes de conduite responsable des entreprises, ou encore des objectifs plus vastes en matière de politique industrielle. En achetant judicieusement ou de manière responsable et durable, les entités publiques peuvent contribuer à la croissance économique et à la création d'emplois, mais aussi améliorer le bien-être social tout en optimisant les ressources disponibles (Commission Européenne, 2011[1]).

Le concept du meilleur rapport qualité/prix a évolué, intégrant de plus en plus de critères(au-delà des aspects financiers)qui ont un impact positif sur la société, l'économie, l'environnement et le bien-être des citoyens (OCDE, 2018[2]). Cependant, les marchés publics ne peuvent pas être utilisés comme levier pour atteindre simultanément tous les objectifs des politiques publiques. Une méthodologie spécifique doit être mise en place afin de mener cette analyse. Pour ce faire, l'Organisation de coopération et de développement économiques (OCDE) recommande aux pays d'entreprendre une série de mesures :

1. Évaluer l'intérêt d'un recours aux marchés publics en tant que méthode pour contribuer à la réalisation d'OPP.

2. Développer une stratégie adaptée pour l'intégration des objectifs politiques secondaires aux systèmes de passation de marchés publics.

3. Employer une méthodologie d'évaluation d'impact adaptée afin d'évaluer dans quelle mesure la passation de marchés publics permet de contribuer à la réalisation des objectifs secondaires.

Parmi les politiques les plus couramment utilisées dans le cadre des marchés publics :

1. Les achats publics environnementaux ou verts concernent les produits et services moins dommageables pour l'environnement lorsque l'on tient compte de l'ensemble de leur cycle de vie (OCDE, 2012[3]).

2. L'aspect social des marchés publics fait référence à l'achat de biens et de services ayant la capacité de générer des résultats sociaux positifs qui dépassent les exigences légales.

3. Les achats durables représentent les processus par lesquels les entités publiques répondent à leurs besoins en biens, services, travaux et services publics de manière à optimiser le rapport qualité-prix sur l'ensemble du cycle de vie en générant des bienfaits non seulement pour l'entité publique, mais également pour la société et l'économie, tout en réduisant considérablement les impacts négatifs sur l'environnement (UNEP, 2015[4]).

4. Les achats publics d'innovation quant à eux font référence à l'achat de solutions innovantes déjà disponibles sur le marché (les partenariats publics d'innovation [PPI]) ou à l'incitation des entreprises à créer des solutions pour des besoins qui ne sont pas couverts par le marché (achats publics avant commercialisation [APAC]).

5. La conduite responsable des entreprises ou responsabilité sociétale des entreprises (RSE)vise à encourager la contribution positive des entreprises au progrès économique, environnemental et social, et à réduire au minimum les risques $s$ que leurs diverses activités peuvent engendrer (OCDE, 2011[5]).

Ce chapitre analyse la prise en compte des OPP dans les marchés publics en Algérie et fournit des recommandations conformes aux meilleures pratiques internationales. 


\subsection{Développer une stratégie pour la prise en compte des politiques publiques dans les marchés publics}

L'utilisation des marchés publiques comme levier stratégique pour atteindre des OPP nécessite de mettre en place une stratégie globale en concertation avec les différentes parties prenantes. II s'agit en effet d'assurer une cohérence avec les différentes politiques entreprises par le pays conformément aux priorités nationales et aux engagements internationaux. Des approches différentes ont été mises en place par les pays pour la prise en compte des OPP dans les marchés publics : i) la prise en compte des OPP dans le cadre réglementaire régissant les marchés publics; ii) le développement de stratégies spécifiques aux OPP dans les marchés publics ; et iii) la prise en compte des marchés publics dans les politiques générales relatives aux OPP. Le graphique 5.1 décrit les différentes approches adoptées par 41 pays pour la prise en compte des objectifs de développement durable (ODD).

\section{Graphique 5.1. Différentes façons de prendre en compte les ODD dans les marchés publics}

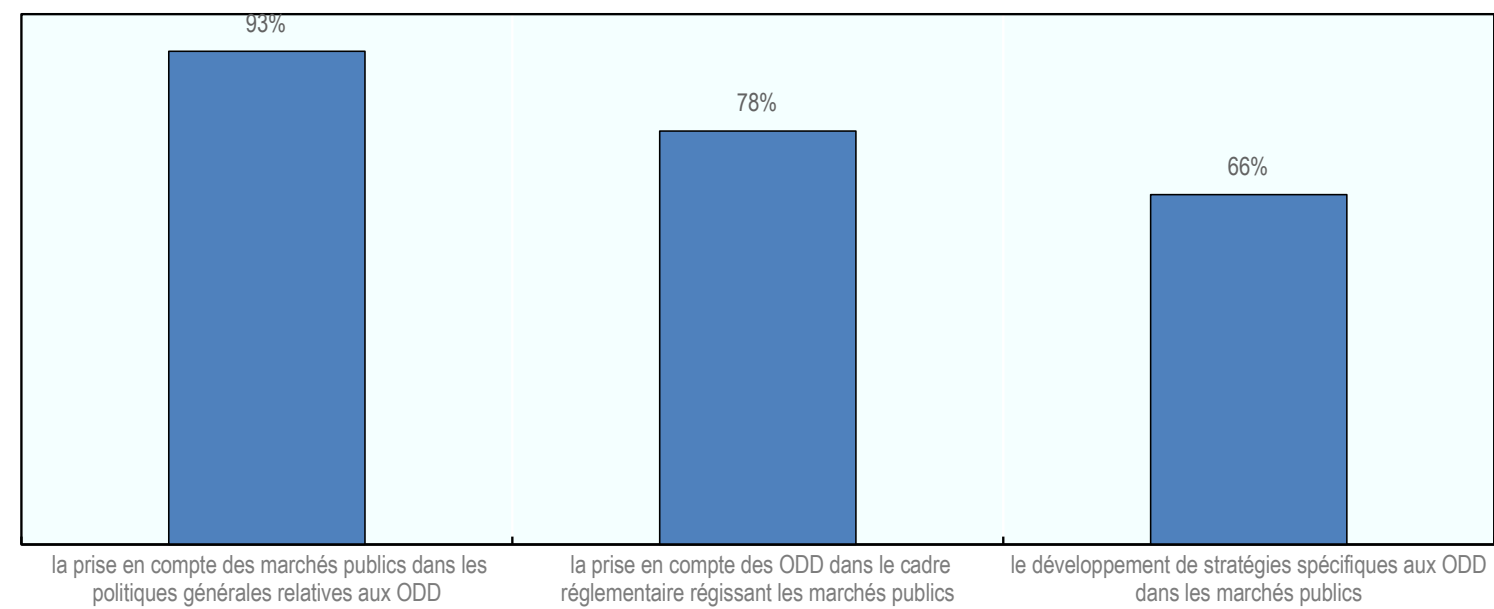

Source : (ONU Environnement, 2017[6]).

\subsubsection{Assurer la cohérence et les synergies entre les différentes politiques en lien avec les marchés publics}

Afin d'assurer une cohérence et une synergie entre les différentes politiques en lien avec les marchés publics, il est nécessaire que ces derniers soient clairement mentionnés et identifiés comme un domaine d'application dans les différents documents officiels, y compris les stratégies et décrets relatifs aux politiques publiques en question. Divers éléments doivent être pris en compte afin d'assurer des résultats tangibles. Par exemple au Canada, la stratégie fédérale sur le développement durable est dupliquée dans plusieurs domaines, y compris dans celui des marchés publics (encadré 5.1). 


\section{Encadré 5.1. Stratégie ministérielle de développement durable 2017-20 du ministère des Services publics et Approvisionnement Canada}

Le ministère des Services publics et de l'Approvisionnement Canada procède à l'achat de biens et de services pour le compte des ministères et organismes du pays en assurant le meilleur rapport qualitéprix pour les Canadiens.

La Stratégie fédérale de développement durable 2016-19 présente les objectifs et les cibles de développement durable du gouvernement du Canada, comme l'exige la Loi fédérale sur le développement durable. Conformément aux objectifs de la loi - intégrer les facteurs environnementaux, sociaux et économiques à la prise de décisions et faire en sorte que de telles décisions soient plus transparentes et responsables devant le Parlement-, le ministère du Services publics et de l'Approvisionnement soutient l'atteinte des objectifs présentés dans la Stratégie fédérale de développement durable au moyen des activités décrites dans la Stratégie ministérielle de développement durable.

Source : (Gouvernement du Canada, 2018[7]).

En termes de développement des PME, l'Algérie a réussi à assurer une cohérence réglementaire entre ses politiques générales de développement des PME et celles spécifiques aux marchés publics. En effet, la Loi $n^{\circ}$ 17-02 du 10 janvier 2017 portant loi d'orientation sur le développement de la PME identifie clairement les marchés publics comme un levier pour le développement des PME : "Au titre de la passation des marchés publics, les services concernés de l'État et de ses démembrements veillent à soumettre une proportion de ces marchés à une concurrence inter-PME selon les conditions et modalités définies par la réglementation en vigueur » (République algérienne, 2017[8]).

En ce qui concerne le développement durable, de nombreux pays ont adopté le Programme de développement durable à l'horizon 2030 (Programme 2030) des Nations Unies qui inclut 17 ODD, également nommés Objectifs mondiaux (voir graphique 5.2), (PNUD, 2018[9]).

\section{Graphique 5.2. Les objectifs de développement durables}

\section{(4) OBJECTIFS ODURABLE}
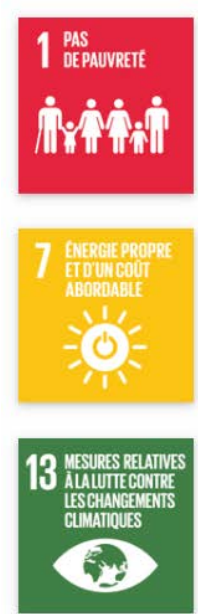

Source : (PNUD, 2018[9]).
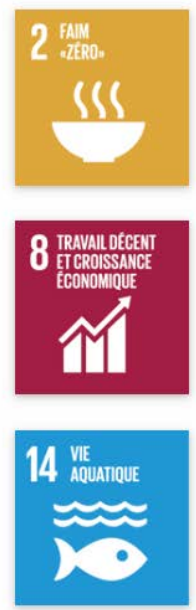
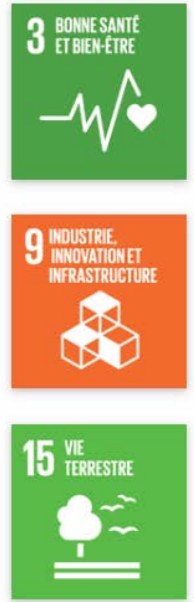
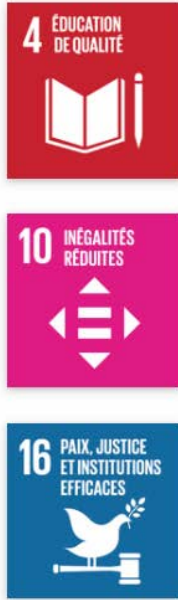
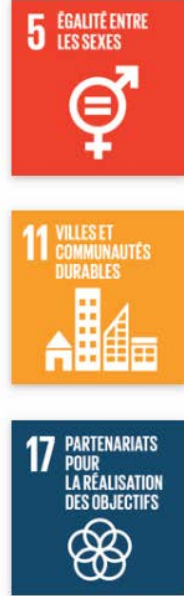
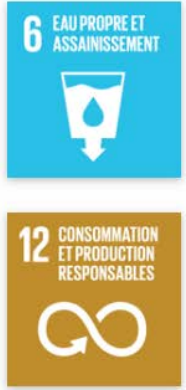

(3)

OBJECTIFS

DEDEVELOPPEMENT 
Chacun de ces objectifs se décline en cibles, dont plusieurs ont un lien direct ou indirect avec les marchés publics. Ainsi, l'objectif 12.7 vise à promouvoir des pratiques durables dans le cadre de la passation des marchés publics, conformément aux politiques et priorités nationales, et l'objectif 16.6 vise à mettre en place des institutions efficaces, responsables et transparentes à tous les niveaux en particulier pour une gestion budgétaire efficace. Cependant, malgré l'adhésion de l'Algérie aux ODD, aucune mesure spécifique n'a encore été prise pour assurer l'alignement des politiques de marchés publics avec les ODD.

Concernant les aspects relatifs au développement durable, l'Algérie s'est engagée par la déclaration de Johannesburg à « promouvoir des politiques de passation des marchés publics qui encouragent la mise au point et la diffusion de biens et services écologiquement rationnels » (Nations Unies, 2002[10]). Cependant, la Stratégie nationale environnementale sous tendue par le Plan national d'action pour l'environnement et le développement durable pour la période 2002-11 développée par le ministère de l'Environnement et des Énergies renouvelables (MEER) n'identifie pas les marchés publics comme un levier stratégique pour atteindre des ODD, y compris pour les aspects environnementaux. D'après les discussions qui se sont tenues lors des missions exploratoires de l'OCDE, il en va de même pour la Stratégie Nationale de l'environnement et du développement durable, actuellement en cours de finalisation.

Le gouvernement, le patronat et l'Union générale des travailleurs algériens ont signé en 2014 le Pacte national économique et social de croissance qui vise la préservation des acquis sociaux, l'édification d'une économie nationale émergente, diversifiée et créatrice de richesse et d'emplois durables, promeut la collaboration entre le gouvernement et la société civile, et fixe des objectifs à horizon 2019 en matière de développement durable (République algérienne démocratique et populaire, 2014[11]). Dans ce texte, les parties prenantes reconnaissent la nécessité de favoriser une croissance durable à travers les politiques publiques. Aucune allusion n'est cependant faite aux marchés publics. Par ailleurs, bien que la société civile soit clairement mentionnée dans le pacte comme l'une des parties prenantes, aucune organisation représentant la société civile ne l'a officiellement signé.

En termes d'innovation, l'Algérie est classée 110e au classement du Global Innovation Index (GII, 2018 ${ }_{[12]}$ ). Suite à un programme de jumelage institutionnel entre l'Algérie et l'Union européenne (UE), le ministère de l'Industrie et des Mines (MIM) élabore actuellement une stratégie d'innovation industrielle (Programme P3A, 2018[13]), mais les informations détaillées sur cette stratégie ne sont pas disponibles. D'après les missions exploratoires de l'OCDE, cette stratégie n'identifie pas directement les marchés publics comme un levier d'innovation.

De plus, bien que le cadre réglementaire régissant les marchés publics consacre plusieurs articles à la promotion de la production nationale et de l'outil national de production, la politique gouvernementale dans le domaine de l'industrie et des mines ne mentionne pas clairement les marchés publics comme un levier pour promouvoir la production nationale.

La responsabilité sociétale des entreprises (RSE) est également un enjeu majeur à intégrer dans le cadre réglementaire des marchés publics pour plusieurs raisons :i) d'après plusieurs études, les entreprises avec une responsabilité sociale élevée obtiennent de meilleurs résultats financiers (Eccles, 2012[14]); et ii) l'État doit s'assurer que ses fournisseurs et leurs chaînes logistiques respectent les droits de l'Homme et des travailleurs, proscrivent le travail des enfants et mènent des actions pour prévenir les dommages environnementaux. Consciente du rôle que peut jouer le secteur privé pour avancer dans ce domaine, l'OCDE a développé les « Principes directeurs à l'intention des entreprises multinationales » qui sont des recommandations que les gouvernements adressent à toutes les entreprises pour encourager leur contribution positive au progrès économique, environnemental et social, et réduire au minimum les difficultés que leurs diverses activités peuvent engendrer (OCDE, 2011[5]). En Algérie, la RSE reste une démarche très limitée malgré quelques actions organisées par des associations et instituts tels que le Cercle d'action et de réflexion autour de l'entreprise, l'Institut algérien de gouvernance d'entreprise, 
Hawkama El Djazaïr, et l'Observatoire de la responsabilité sociétale des entreprises. Elle se développe progressivement mais inégalement selon les secteurs et la taille des entreprises (ORSE, s.d. ${ }_{[15]}$ ).

Par ailleurs, d'après une étude du département d'État américain, de nombreuses entreprises algériennes considèrent les programmes sociaux comme relevant de la responsabilité des gouvernements et n'intègrent pas de telles activités dans leurs processus décisionnels. Bien que les entités publiques accueillent favorablement les activités liées à la RSE des entreprises étrangères, le gouvernement ne les prend pas en compte dans les décisions en matière de marchés publics (U.S. Department of State, 2018[16]).

Étant donné les engagements forts du gouvernement algérien, en particulier au niveau international, ce dernier gagnerait à assurer une cohérence entre les OPP et le cadre réglementaire régissant les marchés publics. Par ailleurs, il gagnerait à développer des stratégies identifiant clairement les marchés publics comme un levier stratégique pour la mise en place des différentes politiques publiques : développement durable, développement des PME, innovation, responsabilité sociétale des entreprises.

\subsubsection{L'engagement et la participation des différentes parties prenantes à l'atteinte d'objectifs de politiques publiques via les marchés publics}

La participation de toutes les parties prenantes à l'élaboration d'une stratégie visant à utiliser les marchés publics comme un levier afin d'atteindre d'autres OPP est fondamentale pour le bon fonctionnement du système de passation des marchés publics (OCDE, 2015[17]). Ceci est important afin : i) d'assurer une cohérence au niveau des politiques nationales; ii) d'assurer le partage des connaissances et des expertises dans certains domaines que l'entité en charge du système de passation de marchés publics ne maîtrise pas nécessairement; et iii) d'assurer une mise en place effective de ces politiques et une sensibilisation de toutes les parties prenantes.

En Algérie, plusieurs entités développent et mettent en place les stratégies et politiques en matière de politiques publiques. Le MEER est chargé du développement de la politique de développement durable et des énergies renouvelables. En ce qui concerne les politiques liées à l'innovation, plusieurs entités jouent un rôle fondamental : le ministère de l'Industrie et des Mines, mais aussi l'Agence nationale de soutien à l'emploi des jeunes (Ansej), l'Agence nationale de développement de la PME (Andpme), et l'Institut national algérien de la propriété intellectuelle (Inapi). Au sein de la Direction générale de la compétitivité industrielle du MIM se trouve la Division de l'Innovation qui est chargée notamment de :i) développer les capacités de l'innovation industrielle ; ii) proposer, en relation avec les parties concernées, les politiques et les programmes de développement des capacités d'innovation et suivre leur mise en œuvre ; et iii) contribuer à la mise en place du système national de l'innovation dans le domaine industriel (Ministère de l'Industrie et des Mines, Algérie, s.d.[18]). L'Ansej, placée sous tutelle du ministère de l'Emploi, met en place des politiques publiques pour réguler la création de PME en fonction des secteurs d'activité et dispose d'outils permettant aux PME d'être éclairées sur l'accès aux marchés publics. Par ailleurs, la Caisse de garantie des crédits d'investissement (CGI) soutient la création et le développement des PME en leur fournissant un accès simplifié au crédit et en garantissant aux banques et aux établissements de crédit les risques de défaillance de remboursement de crédits d'investissement. L'Inapi a également pour mission de promouvoir, développer et renforcer la capacité inventive et innovatrice par des mesures d'incitation matérielles et morales.

La participation et l'engagement des entités chargées du développement et de l'implémentation des politiques publiques à l'élaboration du cadre réglementaire régissant les marchés publics est un élément clé pour l'implémentation effective de ces politiques. Inversement, les entités chargées du cadre régissant les marchés publics doivent contribuer à l'élaboration des stratégies et cadre régissant les politiques publiques (voir graphique 5.3). En effet, en Algérie lors de l'élaboration du décret portant sur la réglementation des marchés publics et des délégations de services publics (DMP), la participation des différentes parties prenantes a été limitée pour plusieurs raisons, notamment le manque de sensibilisation 
au rôle fondamental que peuvent jouer les marchés publics pour l'atteinte d'OPP, mais aussi une culture de la coordination qui n'est pas assez ancrée dans les habitudes de travail. Des acteurs comme le MIM ont contribué au décret avec l'insertion de plusieurs dispositions relatives aux PME. D'autres acteurs, tels que le MEER, n'ont pas apporté de contributions majeures au décret, ce qui pourrait expliquer le nombre limité de dispositions en lien avec les aspects développement durable.

\section{Graphique 5.3. Coordination entre les entités chargées du système de passation des marchés publics et celles chargées des politiques publiques}

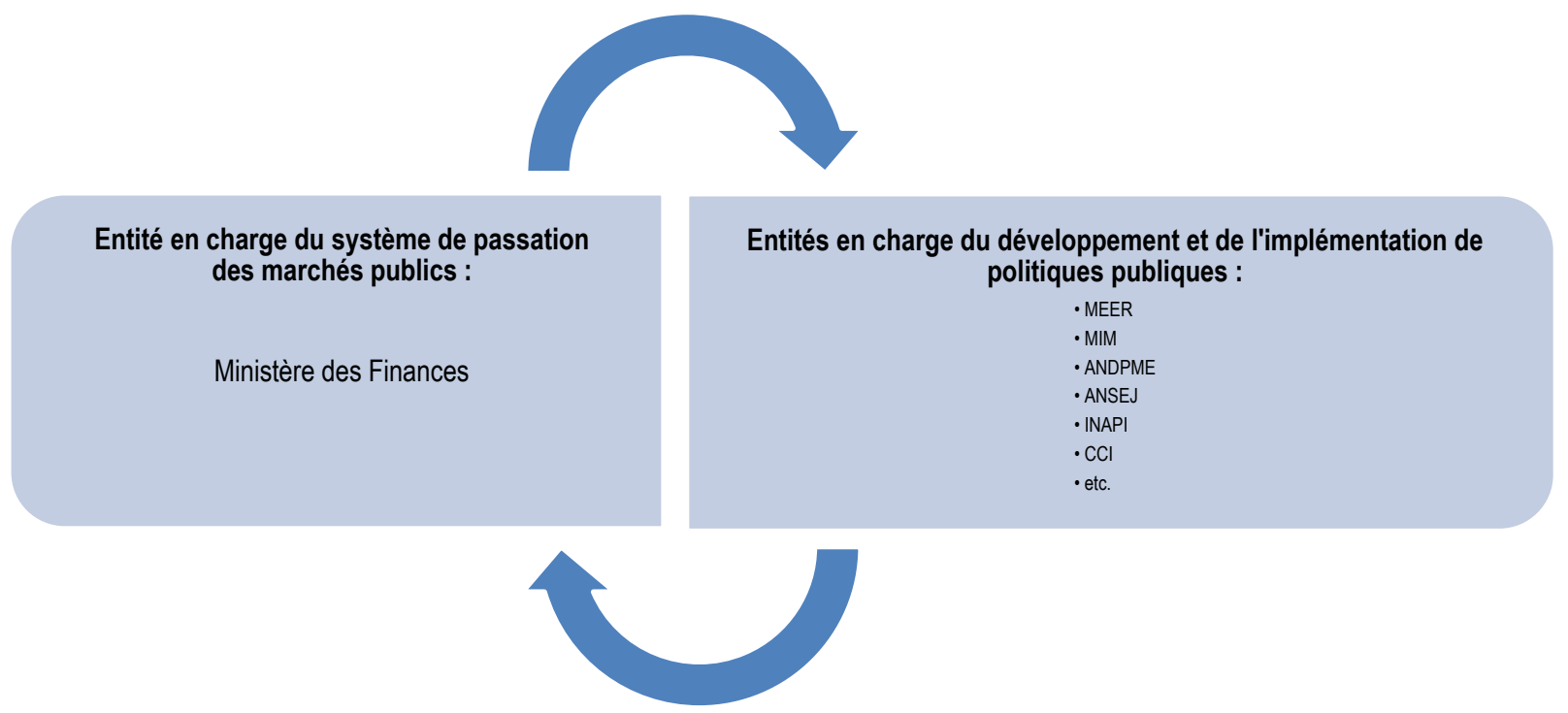

La coordination et le dialogue entre les différentes entités publiques est un facteur clé de réussite de la prise en compte des OPP dans les marchés publics. D'ailleurs, parmi les engagements des pouvoirs publics dans le Pacte national économique et social de la croissance figure la nécessité de « veiller à densifier la coordination et la concertation continues avec les partenaires sociaux et la société civile dans la mise en œuvre stratégique des politiques économiques, notamment en matière de politiques publiques, budgétaires et financières » (République algérienne démocratique et populaire, 2014[11]).

De plus, le développement de stratégies ne peut se faire sans une participation active du secteur privé. En effet des discussions avec les chambres de commerce et les associations professionnelles et patronales sont nécessaires pour le développement et la mise en place effective des politiques publiques. Le secteur privé doit avoir la capacité, ou devrait être préparé à la mise en place, de ces politiques. Des mécanismes de participation doivent donc être mis en place (voir chapitre 1). Les informations collectées lors de la mission exploratoire ont souligné la participation limitée du secteur privé. Ceci est d'autant plus important que toutes les politiques qui visent les marchés publics impactent directement le secteur privé. Le gouvernement Algérien devrait donc assurer l'engagement et la participation des différentes parties prenantes à l'élaboration d'une stratégie visant à utiliser les marchés publics comme un levier stratégique pour l'atteinte d'OPP. Cette coordination est l'une des missions de l'Autorité de régulation des marchés publics (ARMP), dont le rôle est " d'élaborer et de suivre la mise en œuvre de la réglementation des marchés publics et des délégations de service public » mais aussi « d'informer, de diffuser et de vulgariser tous documents et informations relatifs aux marchés publics et aux délégations de service public ". Cependant cette autorité n'a toujours pas été créée. Il est donc nécessaire de la mettre en place dans les plus brefs délais afin de garantir la prise en compte des objectifs des politiques publiques dans les marchés publics. 


\subsection{Définir un cadre pour la prise en compte des politiques publiques dans les marchés publics}

La prise en compte effective des OPP dans les marchés publics rencontre des obstacles, comme par exemple l'idée que les biens et services dits écologiques ou responsables seraient plus coûteux, ou encore le manque de connaissances techniques et d'expertise sur les façons d'intégrer les OPP dans les procédures de marchés publics (OCDE, 2015[19]). II est donc nécessaire de mettre en place un cadre et une stratégie pour la prise en compte des OPP dans les marchés publics, mais aussi une approche globale(voir encadré 5.2).

\section{Encadré 5.2. Dimensions pour la prise en compte des politiques publiques dans les marchés} publics

La prise en compte des politiques publics dans les marchés publics nécessite une approche globale incluant les dimensions suivantes:

1. Mettre en place des stratégies et un cadre réglementaire pour la prise en compte des OPP dans les procédures d'achats des entités publiques avec des objectifs clairs à atteindre.

2. Planifier la prise en compte des OPP, notamment analyser les capacités du marché et les solutions techniques disponibles, tout en mesurant les coûts et bénéfices de la prise en compte de ces objectifs.

3. Inclure des standards internationaux et nationaux dans les spécifications techniques, les critères de sélection et de choix, mais également dans les clauses contractuelles relatives à l'exécution des marchés.

4. Professionnaliser les acheteurs publics et renforcer leur capacité, leur compétence et leur expertise dans le domaine des OPP.

5. Sensibiliser les acheteurs publics, les entreprises et la société civile aux bienfaits de l'intégration des OPP dans les marchés publics.

6. Suivre et mesurer la prise en compte des politiques publiques dans les marchés publics et assurer une rétroaction dans les politiques et la réglementation.

7. Lorsque nécessaire, prévoir un budget spécifique pour favoriser la mise en place de certaines politiques publiques telles que l'innovation.

8. Utiliser des systèmes d'achat électroniques et des outils informatiques pour accompagner la mise en place des politiques publiques dans les marchés publics.

9. Mettre en place un système de management des risques.

Source : adapté de (OCDE, 2015[19]) ; (OCDE, 2017[20]).

\subsubsection{Définir des objectifs clairs de politiques publiques à atteindre dans les marchés publics}

Le soutien à l'implémentation effective des politiques publiques dans les marchés publics peut être facilité par la mise en place d'objectifs quantitatifs ou qualitatifs (OCDE, 2017[20]). Ces objectifs donnent une indication claire de l'engagement d'un gouvernement pour une politique publique donnée, c'est pourquoi de nombreux pays les ont mis en place. Ces objectifs varient très largement d'un pays à l'autre et peuvent être développés au niveau national, régional et local. De plus, certains pays ont développé des objectifs 
concrets mesurables, alors que dans d'autres pays les objectifs ont plutôt une fonction de signalisation incarnant une ambition. Le développement d'objectifs clairs est un élément clé pour mesurer la contribution des achats publics à la mise en œuvre des politiques publiques d'un point de vue qualitatif et quantitatif (Commission Européenne, 2011 ${ }_{[1]}$ ). Par exemple, le gouvernement wallon (Belgique) a adopté le Plan d'actions achats publics responsables 2017-19 qui a pour objectif que, d'ici 2020, les marchés publics passés en Wallonie soient $100 \%$ responsables face aux défis sociétaux (Service Public de Wallonie, $\left.2017_{[21]}\right)$. L'encadré 5.3 présente le plan d'action pour les achats durables en France qui prévoit des objectifs à atteindre en termes de développement durable.

\section{Encadré 5.3. Le plan national d'action pour les achats publics durables à horizon 2020 en France}

Le plan national d'action pour les achats publics durables est une feuille de route permettant de s'attaquer de façon pragmatique, et sur la durée, aux obstacles qui ralentissent la montée en puissance de l'achat public durable : convaincre les décideurs, accompagner les acheteurs, dialoguer avec les autres acteurs essentiels que sont les entreprises et leurs organisations représentatives, montrer, démontrer, valoriser, évaluer, etc.

Les objectifs stratégiques fixés par le document le sont à l'horizon 2020 :

- $30 \%$ des marchés publics doivent avoir une disposition environnementale.

- $25 \%$ des marchés publics doivent avoir une disposition sociale.

- $100 \%$ des marchés font l'objet d'une analyse approfondie, visant à définir si les objectifs du développement durable peuvent être pris en compte dans le marché.

- $60 \%$ des organisations publiques sont signataires de la charte pour l'achat public durable.

- $80 \%$ des organisations réalisant des achats de papier, d'appareils d'impression, de fournitures, de mobilier, de vêtements, de matériel de bureautique prennent en compte la fin de vie de ces produits.

Source : (Ministère de la Transition écologique et solidaire, France, 2018[22]].

L'utilisation des marchés publics pour atteindre des objectifs d'innovation est également l'une des politiques publiques pour laquelle les pays ont déterminé des objectifs clairs à atteindre (voir l'encadré 5.4 pour des exemples concrets). 
Encadré 5.4. Exemples d'objectifs mis en place par divers pays en matière d'innovation dans les marchés publics

Exemples de pays ayant des objectifs quantitatifs d'achats innovants :

- Finlande : le programme du gouvernement 2015 inclut un objectif de $5 \%$ d'achats innovants dans les marchés publics.

- France : les PME doivent atteindre $2 \%$ d'achats innovants en 2020.

- Pays-Bas : ambition de dépenser $2.5 \%$ en achats innovants.

- Espagne : un objectif de $3 \%$ de nouvel investissement pour les achats innovants.

- Corée du Sud : les autorités centrales ou locales et les entreprises publiques doivent réaliser $20 \%$ de leurs achats en biens spécifiques pour lesquels des nouvelles technologies ou des produits certifiés sont disponibles.

Exemples de pays ayant des objectifs qualitatifs d'achats innovants :

- Pays-Bas, Belgique/Flandres : objectifs indicatifs afin de stimuler les achats innovants.

- Danemark : promouvoir les achats publics performants et innovants.

- Turquie: augmenter la part des entreprises nationales opérant dans les secteurs de technologies de pointe dans les marchés publics.

Source : (OCDE, 2017[20]).

En Algérie, mis à part pour les PME, aucun objectif clair n'est défini concernant la contribution des marchés publics aux politiques publiques. Le gouvernement algérien devrait donc prévoir des objectifs de politiques publiques à atteindre dans le cadre des stratégies à développer pour l'utilisation des marchés publics comme un levier stratégique. L'atteinte d'objectifs pourrait être imposée progressivement afin de préparer et accompagner le secteur privé et les entités publiques à prendre en compte et répondre à ces objectifs.

\subsubsection{Un cadre réglementaire qui encourage la prise en compte du développement durable et de l'innovation}

Les entités publiques peuvent utiliser différents mécanismes afin d'intégrer des aspects de développement durable ou d'innovation dans les marchés publics. Ces mécanismes peuvent couvrir tout le cycle de passation des marchés publics, de la préparation des appels d'offres à la fin d'exécution du contrat. Cependant tout mécanisme devrait être conforme au cadre réglementaire et aux stratégies relatives aux marchés publics. Afin d'assurer une prise en compte effective des différents OPP, il est nécessaire de s'assurer d'une collecte des besoins appropriée et d'entreprendre une étude de marché approfondie destinée à établir si le marché a la capacité de répondre aux exigences des politiques publiques. Le graphique 5.4 présente les éléments à prendre en compte pour insérer les aspects développement durable et innovation dans le cadre réglementaire des marchés publics. 
Graphique 5.4. Les éléments à prendre en compte pour insérer le développement durable et l'innovation dans le cadre réglementaire des marchés publics

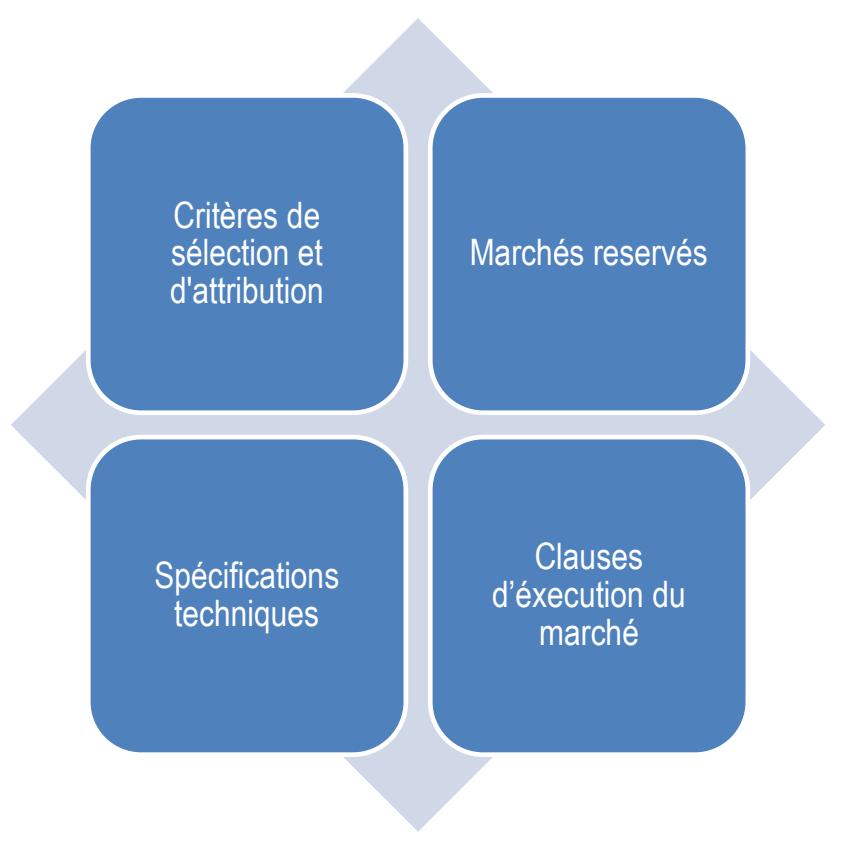

\section{Des critères de sélection et d'attribution, et des clauses d'exécution du marché intégrant les OPP}

Le choix des critères de sélection et d'attribution, ainsi que leurs pondérations respectives ont un impact direct sur la concurrence $\left(\mathrm{OCDE}\right.$, à paraître $\left.{ }_{[23]}\right)$. Ces critères peuvent également permettre la prise en compte des OPP dans les marchés publics. En effet, comme décrit au chapitre 1, les critères de sélection ont pour objectif d'analyser la capacité d'un opérateur économique à exécuter un contrat donné. Lors de cette analyse, une entité publique peut prendre en compte les expériences et exigences en lien avec les politiques publiques, telles que les aspects environnementaux et sociaux (Union Européenne, 2016[24]). Les critères d'attribution permettent d'analyser les offres et d'attribuer un marché. En plus du prix, ces critères peuvent inclure les aspects relatifs à la qualité, aux aspects techniques, au service après-vente, aux aspects esthétiques, mais également aux aspects environnementaux et sociaux (OCDE, 2011 [25]). Les marchés publics peuvent également inclure des clauses spécifiques à la phase d'exécution du contrat qui permettent d'améliorer la prise en compte des éléments environnementaux ou sociaux.

En Algérie, l'article 78 du DMP prévoit plusieurs critères d'attribution pour choisir l'offre économiquement la plus avantageuse, y compris les critères relatifs au « coût global d'acquisition et d'utilisation » et ceux relatifs aux « performances en matière sociale, pour promouvoir l'insertion professionnelle des personnes exclues du marché du travail et des handicapés, et les performances en matière de développement durable ». L'insertion dans le cadre réglementaire de ces critères est considérée comme une bonne pratique internationale pour la mise en place des ODD. Cependant, d'après les entretiens avec différentes parties prenantes, l'utilisation de ces critères reste très limitée et aucune ligne directrice n'est fixée concernant l'utilisation de ces critères. En effet, comme décrit au chapitre 1, le critère d'attribution le plus utilisé est le prix, en raison du manque de sensibilisation des acheteurs et de l'absence de lignes directrices sur l'utilisation de ces critères. De plus, les clauses d'exécution du marché relatives aux aspects sociaux et environnementaux ne semblent pas être utilisées. Le gouvernement algérien gagnerait donc à renforcer les capacités des acheteurs publics à utiliser les critères de sélection et d'attribution et les clauses d'exécution de marchés prenant en compte les enjeux sociaux et environnementaux en organisant des formations et en développant des lignes directrices. 


\section{Des spécifications techniques qui reflètent les OPP}

Les spécifications techniques ont deux fonctions principales. En premier lieu, elles définissent les caractéristiques des services, des fournitures ou des travaux que l'entité publique envisage d'acquérir, ce qui permet de définir le niveau de concurrence sur un marché donné. En second lieu, elles définissent des exigences mesurables qui constituent les critères de conformité « minimaux » (Union Européenne, s.d. [26]). En pratique, elles peuvent comprendre des aspects de performance environnementale, de conception, de sécurité, d'assurance de la qualité ou d'évaluation de la conformité (Union Européenne, s.d.[26]). Par ailleurs, elle peuvent permettre de promouvoir l'innovation dans les marchés publics en mettant en place des mesures, telles que la formation et l'accompagnement des acheteurs publics dans la rédaction de spécifications techniques incluant des exigences en termes de performance ou de standards techniques, ou des certifications qui prennent en compte les aspects d'innovation (OCDE, 2017[20]).

En Algérie, d'après les missions exploratoires, le développement de spécifications techniques incluant des aspects environnementaux, sociaux ou d'innovation est très limité. En effet, le cadre réglementaire ne traite pas de ce sujet en particulier, ce qui n'incite pas les acheteurs publics à prendre en compte ces aspects de politiques publiques dans leur cahier des charges. Le développement par le gouvernement algérien d'une stratégie pour la prise en compte des politiques publiques dans les marchés publics devra donc consacrer également un volet aux spécifications techniques. Par ailleurs, le DMP devrait également traiter de ce sujet.

\section{Pour la mise en place de labels environnementaux en Algérie}

Plusieurs pays membres et non-membres de l'OCDE ont mis en place un système de labélisation environnementale. Ces écolabels, en particulier ceux de type I, doivent être basés sur des critères objectifs et transparents, et être attribués par une tierce partie indépendante. Ils peuvent aider les acheteurs publics dans l'élaboration des spécifications techniques, mais peuvent également être utilisés comme un critère d'attribution ou de qualification des entreprises (Union Européenne, 2016[24]). L'encadré 5.5 fournit les conditions pour l'utilisation de labels environnementaux dans les marchés publics de l'UE.

\section{Encadré 5.5. Les conditions pour l'utilisation de labels environnementaux dans les marchés publics de l'UE}

1. Les labels ne concernent que les critères qui sont liés à l'objet du marché.

2. Les labels sont basés sur des critères objectifs, vérifiables et non-discriminatoires.

3. Les labels sont établis pour des procédures ouvertes et transparentes auxquelles toutes les parties prenantes pertinentes peuvent participer.

4. Les labels sont accessibles à toutes les parties intéressées.

5. Les labels sont définis par une tierce partie indépendante et sur laquelle les opérateurs économiques ne peuvent exercer aucune influence décisive.

Source : (Union Européenne, 2016[24]).

En Algérie, il n'existe pas d'écolabels, cependant un système de labélisation existe puisque l'Institut algérien de normalisation délivre un label national de qualité appelé «TEDJ ». De plus, le Forum des chefs d'entreprises a mis en place le label « Bassma Djazairia » pour les produits ou gamme de produits dont l'essentiel de la valeur ajoutée a été produit en Algérie. Le gouvernement algérien gagnerait donc à mettre en place un système d'écolabels qui serait utile non seulement dans le cadre des marchés publics, mais aussi dans le cadre des achats des citoyens et des entreprises. L'Institut algérien de normalisation pourrait 
être chargé de l'octroi de ce label puisqu'il est responsable de l'élaboration, la publication et la diffusion des normes algériennes (Ministère de l'Industrie et des Mines, Algérie, s.d.[27]).

\subsection{Les marchés publics, un marché considérable pour le développement des PME}

Compte tenu de la structure du secteur privé dans la plupart des pays où les PME représentent la part la plus importante de l'économie, des efforts visant à promouvoir leur croissance économique et leur durabilité, mais aussi leur accès aux différents marchés sont généralement poursuivis. Les marchés publics et la construction d'infrastructures représentent un domaine clé pour l'amélioration de la performance des PME (voir graphique 5.5).

\section{Graphique 5.5. Environnement d'affaires des PME : un cadre conceptuel}

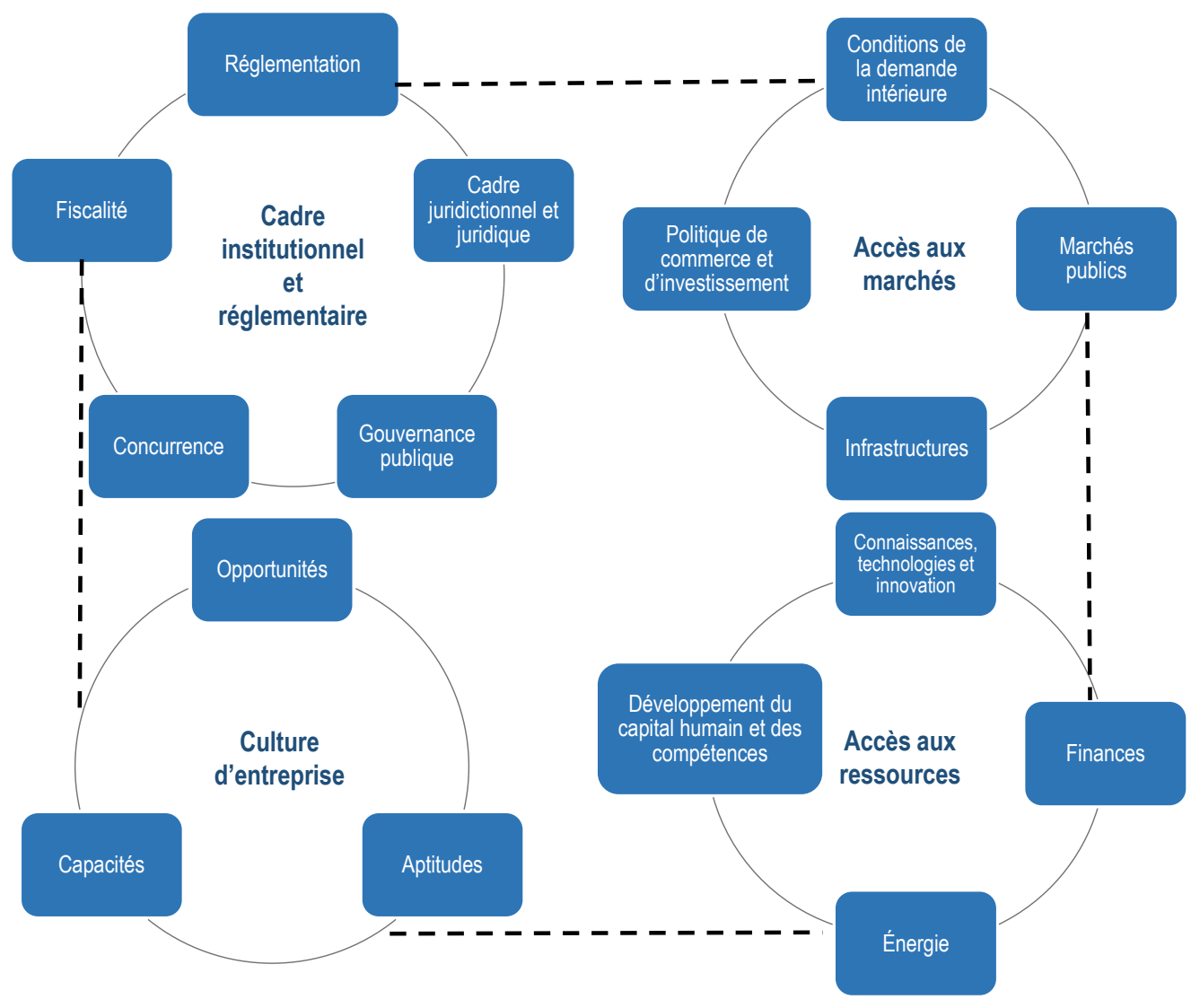

Source : (OCDE, 2017[28)).

Chaque pays possède une définition spécifique des PME. En Algérie, une PME est une « entreprise de production de biens et/ou de services employant une (1) à 250 personnes et dont le chiffre d'affaires annuel n'excède pas 4 milliards DZD (dinars algériens, soit 29 millions EUR [euros]) ou dont le total du bilan annuel n'excède pas 1 milliard DZD (7.3 millions EUR) avec un capital qui n'est pas détenu à $25 \%$ et plus par une ou plusieurs autres entreprises ne correspondant pas à la définition de la PME » (République algérienne, 2017[8]). Le tableau 5.1 ci-dessous fournit le découpage exact entre les différentes catégories de PME. 
Tableau 5.1. Définition de la PME en Algérie

\begin{tabular}{l|c|c|c}
\hline & Chiffre d'affaires en millions DZD & Total du bilan en millions DZD & Nombre de salariés \\
\hline PME & Inférieur à 4 000 & Inférieur à 1 000 & 1 à 250 \\
\hline Très petite entreprise & Inférieur à 40 & Inférieur à 20 & 1 à 9 \\
\hline Petite entreprise & Compris entre 40 et 400 & Compris entre 20 et 200 & 10 à 49 \\
\hline Moyenneentreprise & Compris entre 400 et 4 000 & Compris entre 200 et 1 000 & 50 à 250 \\
\hline
\end{tabular}

Notes : 1) Le critère du chiffre d'affaires ou du total du bilan prime sur le nombre de salarié(s) pour la classification. Le changement de classification ou la perte de qualité de PME a lieu dès lors que des variations interviennent sur deux exercices comptables consécutifs ; 2) Les PME dont le capital social est détenu dans la limite de $49 \%$ par une ou plusieurs sociétés de capital investissement peuvent bénéficier de mesures d'appui.

Source : (République algérienne, $\left.2017_{[8]}\right]$.

Malgré les efforts entrepris ces dernières années pour améliorer l'accès des PME aux marchés publics, celles-ci demeurent confrontées à diverses contraintes, indiquent les responsables politiques et les spécialistes des pays de l'OCDE (voir graphique 5.6).

\section{Graphique 5.6. Principales contraintes d'accès aux marchés publics pour les PME}

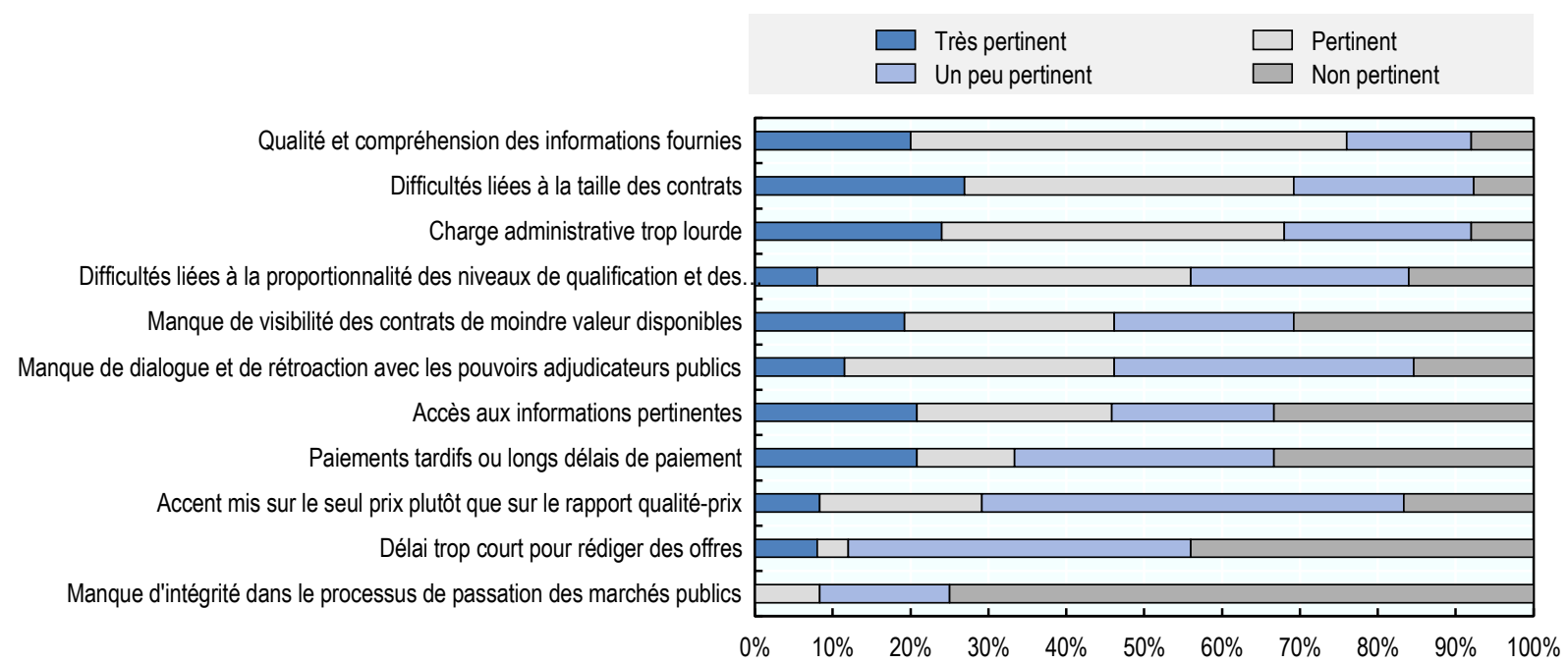

Note : les personnes interrogées ont été invitées à indiquer la pertinence de chaque option dans leur contexte national, sur une échelle de 1 (non pertinent) à 4 (très pertinent). Au total, 30 pays de l'OCDE et 6 pays non-membres de l'OCDE ont répondu à cette enquête.

Source : (OCDE, 2018[29]).

Pour surmonter ces obstacles, deux approches ont été adoptées. La première met en place un système préférentiel pour les PME, ou certains sous-groupes de PME, dans l'attribution de marchés publics. La seconde consiste à créer un environnement concurrentiel avec des mécanismes assurant un traitement égal et équitable des soumissionnaires et des fournisseurs (OCDE, 2018[29]). Ces approches ont été toutes les deux adoptées en Algérie.

\subsubsection{Adapter les programmes de préférences accordées aux PME au contexte algérien}

Les PME font souvent face à diverses difficultés pour accéder aux marchés publics. Les obstacles qu'elles rencontrent lors des appels d'offres publics sont bien connus. En effet, la participation d'une PME aux opportunités des marchés publics requiert des ressources proportionnellement plus élevées que pour une grande entreprises (Yukins et Piga, 2012[30]). C'est pourquoi certains pays ont recours à des mesures 
préférentielles spécifiques à une catégorie d'entreprises donnée. Ces mesures incluent les marchés dit " réservés », mais aussi les préférences en termes de prix proposés (OCDE, 2018[29]).

Cependant, la mise en place de mesures préférentielles spécifiques aux PME confronte les pays à un dilemme : si ces mesures peuvent permettre d'améliorer l'accès des PME aux marchés publics et leur survie à long terme, elles vont toutefois à l'encontre du principe d'égalité de traitement (OCDE, 2018[29]).

Parmi les pays de l'OCDE, seuls la Corée et les États-Unis ont mis en place un système de préférence pour les PME. Des pays de la région MENA ont également mis en place ce type de mesures. Par exemple, en Tunisie il est prévu que les entités publiques doivent attribuer « dans la limite de $20 \%$ » du montant estimé des marchés de travaux, de fourniture de biens, de services et d'études aux petites entreprises (OCDE, 2018[29]).

En Algérie, l'article 87 du DMP prévoit que « lorsque certains besoins des services contractants peuvent être satisfaits par des micro-entreprises, telles que définies par la législation et la réglementation en vigueur, les services contractants doivent, sauf exception dûment justifiée, leur réserver exclusivement ces prestations ". Ainsi chaque entité peut accorder un maximum de $20 \%$ de la commande publique à des micro-entreprises dans la limite de montant maximum spécifié dans le même décret. Cependant, le cadre réglementaire qui définit les PME ne prévoit pas de catégorie dite « micro-entreprise ». Elle pourrait certainement correspondre á la catégorie « très petite entreprise » prévue dans la Loi d'orientation sur le développement de la petite et moyenne entreprise. Le gouvernement algérien devrait donc harmoniser le vocabulaire utilisé dans les différents cadres réglementaires afin d'assurer une cohérence des politiques et des termes utilisés.

De plus, toute mesure introduite dans le cadre réglementaire doit être basée sur des études détaillées. Cependant d'après les discussions avec les différentes parties prenantes lors de la mission exploratoire, il semblerait qu'aucune étude n'ait été réalisée afin de déterminer le pourcentage le plus adéquat et d'analyser l'impact de cette mesure. Le gouvernement algérien devrait donc considérer la réalisation d'études afin de déterminer si cette mesure a un impact positif sur le développement des PME, et le cas échéant déterminer le pourcentage le plus adéquat à prévoir.

\subsubsection{Mettre en place des mécanismes réglementaires visant à améliorer l'accès des $P M E$ à la commande publique}

L'un des obstacles à l'accès des PME aux marchés publics est lié à la taille du marché. Afin de lever cet obstacle, plusieurs mécanismes peuvent être mis en place tels que l'allotissement, le groupement de commande ou la sous-traitance.

\section{Justifier le non recours à l'allotissement}

Le recours à d'allotissement est l'une des mesures les plus couramment utilisées pour faciliter l'accès des PME aux opportunités de marchés publics. En effet, $83 \%$ des pays de l'OCDE ont mis en place des mesures pour encourager l'allotissement des marchés (voir graphique 5.7). Grace à ces stratégies qui permettent de prévoir des marchés de tailles plus adéquates pour les PME, ces dernières peuvent accéder à davantage d'opportunités de marchés publics. En effet, par l'allotissement, la taille des marchés pourrait mieux correspondre à la capacité de production des PME et les spécifications techniques de chaque lot pourraient davantage correspondre à leur secteur d'expertise et de spécialisation (OCDE, 2018[29]). 
Graphique 5.7. Pays qui encouragent l'allotissement des marchés

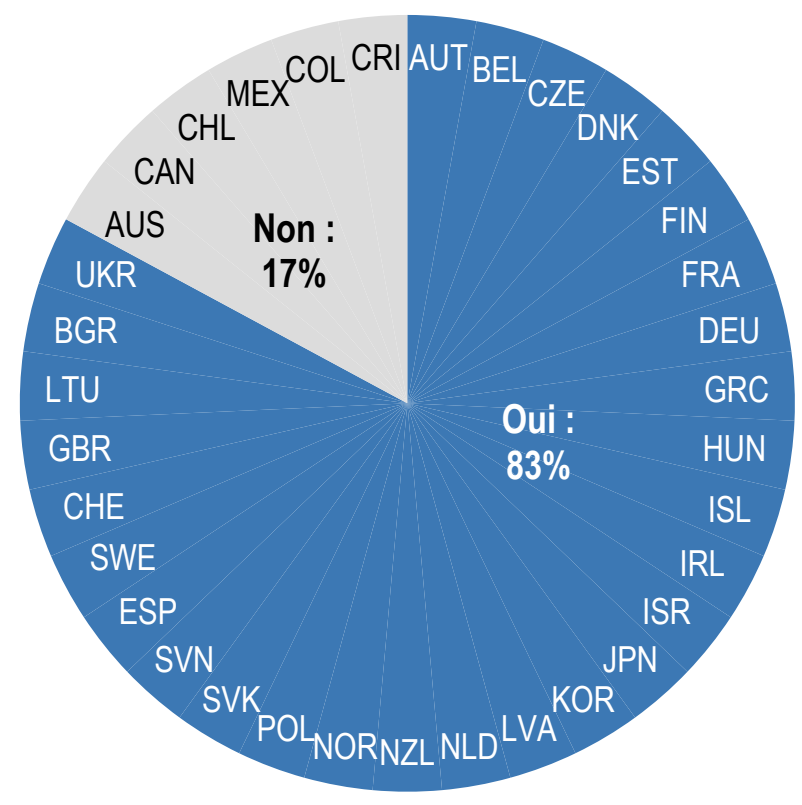

Source : (OCDE, 2018[29]).

Le cadre réglementaire régissant les marchés publics prévoit l'allotissement des marchés par des lots uniques ou séparés. Le recours à l'allotissement est à effectuer à " chaque fois que cela est possible, en fonction de la nature et de l'importance du projet, et de la spécialisation des opérateurs économiques, doit tenir compte des avantages économiques, financiers et/ou techniques procurés par cette opération " (art. 31 du DMP). Cependant, contrairement aux pratiques d'autres pays, y compris ceux de l'OCDE, en Algérie chaque entité publique doit " motiver son choix » d'allotir un marché. La mesure prévue dans le cadre réglementaire algérien risque de ne pas encourager les entités publiques à recourir à l'allotissement de leurs marchés. L'encadré 5.6 présente la stratégie d'allotissement prévue dans les pays de l'UE qui sont tenus de justifier le non recours à l'allotissement. Afin d'améliorer l'accès des PME aux marchés publics, le gouvernement algérien devrait imposer l'allotissement de tous les marchés, tout en prévoyant une justification détaillée de la part des entités publiques lorsque l'allotissement n'est pas possible. 


\section{Encadré 5.6. L'allotissement dans les pays de l'UE}

78) La passation des marchés publics devrait être adaptée aux besoins des PME. [...]. À cet effet, et afin de renforcer la concurrence, les pouvoirs adjudicateurs devraient en particulier être encouragés à diviser en lots les marchés importants. Cette division pourrait se faire sur une base quantitative, en faisant mieux correspondre la taille des différents marchés à la capacité des PME, ou sur une base qualitative, en fonction des différentes branches d'activité et spécialisations concernées, afin d'adapter plus étroitement le contenu de chaque marché aux secteurs de spécialisation des PME, ou selon les différentes phases successives du projet. [...]

Le pouvoir adjudicateur devrait avoir l'obligation d'examiner l'opportunité de diviser les marchés en lots tout en demeurant libre de prendre sa décision de façon autonome sur la base de tout motif qu'il juge pertinent, sans faire l'objet d'un contrôle administratif ou judiciaire. Lorsque le pouvoir adjudicateur décide qu'il ne serait pas indiqué de diviser le marché en lots, le rapport individuel ou les documents de marché devraient comporter une mention des principaux motifs justifiant son choix. À ce titre, le pouvoir adjudicateur pourrait par exemple faire valoir qu'il estime que cette division risquerait de restreindre la concurrence ou de rendre l'exécution du marché excessivement coûteuse ou difficile sur le plan technique, ou que la nécessité de coordonner les contractants des différents lots pourrait compromettre gravement la bonne exécution du marché.

Source : (Union Européenne, 2014[31]).

L'un des risques de l'allotissement est de fractionner les besoins afin d'échapper aux procédures à appliquer et aux seuils de compétence des commissions des marchés (OCDE/SIGMA, 2016[32]). Conformément aux bonnes pratiques internationales, l'article 31 du DMP interdit le fractionnement des besoins. Cependant, d'après les missions exploratoires de l'OCDE, ces cas de figure peuvent se présenter et ne font pas l'objet de contrôles de la commission des marchés compétente, les marchés en dessous des seuils d'appel d'offres faisant l'objet d'une procédure adaptée (voir chapitre 1). L'Algérie devrait donc renforcer les contrôles pour les procédures en dessous des seuils afin de s'assurer que les besoins ne soient pas fractionnés.

\section{Mettre en place des mécanismes visant à encourager la cotraitance}

Un autre mécanisme qui permet aux PME d'accéder aux marchés publics est la cotraitance ou le groupement d'entreprises. La cotraitance consiste à former un groupe d'opérateurs économiques qui soumissionnent conjointement sur des marchés importants et complexes, dont l'exécution dépasse leurs capacités individuelles (OCDE/SIGMA, 2016 $6_{[33]}$ ). En effet, il peut arriver qu'une entreprise ne puisse pas exécuter seule la totalité du marché, ou qu'elle n'ait pas la capacité administrative ou financière pour l'exécuter. La cotraitance est une pratique courante dans les pays de l'OCDE lorsqu'elle ne va pas à l'encontre du droit et de la concurrence (OCDE, 2018 [29] . En effet, la cotraitance ne devrait pas être autorisée si chacune des entreprises à la capacité économique, financière et technique de répondre à un marché donné (OCDE, 2011 [34]).

En Algérie, conformément aux bonnes pratiques internationales, le DMP prévoit la possible de former un groupement momentané d'entreprises solidaires ou conjointes. Cependant, du fait de l'absence de système de collecte d'information, aucune donnée n'a pu être partagée avec les experts de l'OCDE sur la fréquence d'utilisation de cette disposition et les difficultés rencontrées par les entreprises qui souhaiteraient répondre conjointement à un marché public. Le système pourrait être plus efficace si un espace était disponible afin de faciliter le contact entre les différentes entreprises. L'encadré 5.7 présente 
un nouveau service mis en place par la Direction des achats de l'État en France qui facilite le contact entre de potentiels cotraitants. Le gouvernement algérien pourrait promouvoir la cotraitance auprès du secteur privé et créer un espace ou un outil visant à faciliter le contact entre les entreprises désireuses de former un groupement afin de répondre à un appel d'offres donné.

\section{Encadré 5.7. La bourse à la cotraitance en France}

Un nouveau service de bourse à la cotraitance est proposé sur le portail « entreprises » de la plateforme des achats de l'État (Place) pour faciliter les contacts entre entreprises avant de répondre à des marchés de l'État sous la forme de groupement momentané d'entreprise (GME).

Avec cet outil, la Direction des achats de l'État veut faciliter pour les entreprises, et particulièrement les PME, l'accès à des marchés publiés par les ministères et leurs établissements publics, I'UGAP (une centrale d'achat publique) et les Chambres de commerce et d'industrie, même si elles n'ont ni la taille, ni l'ensemble des compétences requises pour répondre seules à ces marchés.

Source : (Direction des achats de l'État (DAE), s.d.[35]).

\section{Autoriser la sous-traitance dans les marchés de fourniture}

Pour certains marchés complexes ou de taille importante, une PME ne peut être l'attributaire principal d'un marché ou répondre en groupement avec d'autres entreprises en raison de ses capacités limitées. Tout comme la cotraitance, la sous-traitance est donc une solution qui permet la participation des PME à la commande publique (OCDE/SIGMA, 2016[33]). Cependant, l'une des problématiques principales rencontrées par les sous-traitants dans le cadre des marchés publics est liée au paiement (OCDE, 2018[29]).

En Algérie, la sous-traitance dans les marchés publics est utilisée comme un outil de promotion de la production nationale. Plusieurs bonnes pratiques ont été mises en place, telles que le paiement direct des sous-traitants, la limitation de la part du marché pouvant être sous-traitée (40\%), et la prise en compte de la capacité des sous-traitants dans l'évaluation des capacités du soumissionnaire principal.

Le DMP, autorise la sous-traitance dans la passation des marchés publics, quel que soit la nature de la prestation à réaliser, exception faite des marchés de fournitures, dont le caractère est courant. Ces derniers peuvent inclure des services connexes, tels que la livraison, qui pourraient être sous-traités (OCDE, 2019[36]).Le gouvernement algérien gagnerait donc à revoir cette disposition afin d'améliorer l'efficience du système.

\section{Diminuer les barrières financières pour l’accès des PME aux marchés publics}

L'une des barrières à l'accès des PME aux marchés publics réside dans des exigences financières disproportionnées dans les critères de sélection, mais aussi en matière de garanties financières demandées dans le cadre de la soumission des marchés (OCDE, 2018[29]). En ce qui concerne les exigences financières liées aux critères de sélection, le cadre réglementaire algérien prévoit plusieurs dispositions pour les PME nouvellement créées. Par exemple, le DMP mentionne que «le montant minimum du chiffre d'affaires, le nombre de bilans et l'absence de références similaires ne doivent pas être des motifs pour rejeter les candidatures des PME, telles que définies par la législation et la réglementation en vigueur, nouvellement créées, sauf si l'objet et la nature du marché l'exigent ». Toutefois, aucune ligne directrice n'a été développée afin d'assurer que les acheteurs publics demandent des exigences financières proportionnelles au contrat. Le développement de telles lignes directrices pourrait aider les entités publiques algériennes à choisir des critères de qualification et des exigences financières proportionnelles au marché qui ne nuisent pas à l'accès des PME. 
Concernant les garanties financières, en Algérie les cautions de soumission et de bonne exécution pourraient être revues à la baisse pour améliorer l'accès aux marchés publics. Le DMP dispense les artisans d'art et les micro-entreprises de droit algérien de présenter une caution de bonne exécution lorsqu'ils interviennent dans des opérations publiques de restauration de biens culturels. Cette disposition vise donc uniquement une catégorie de PME pour une catégorie de service spécifique. L'accès des PME aux marchés publics pourrait donc être amélioré soit en généralisant les dispenses de cautions pour toutes PME, quels que soient les marchés publics auxquels elles soumissionnent, soit en diminuant leurs pourcentages.

De plus, il est de la responsabilité des entités publiques de payer à temps leur fournisseur. En effet des retards de paiement font peser des risques financiers majeurs sur les PME pouvant aller jusqu'à leur faillite (OCDE, 2018[29]). Le chapitre 1 mentionne les lacunes du système algérien en termes de paiement. Ces lacunes ont des répercussions directes sur les PME. Le respect et l'amélioration du délai de paiement pour toutes les entreprises, en particulier pour les PME, est un élément décisif pour améliorer leur accès aux marchés publics. En plus du respect du délai de paiement, certains pays de l'OCDE ont mis en place des initiatives afin de soutenir financièrement les PME titulaires de marchés publics (voir encadré 5.8). Le gouvernement algérien devrait mener des actions concrètes afin d'assurer le respect des délais de paiement et de mettre en place de mécanismes qui permettent de le réduire.

\section{Encadré 5.8. Initiatives de soutien financier aux PME en France et en Corée}

\section{Affacturage inversé en France}

En plus du versement d'avances prévu par la loi française sur les marchés publics, l'UGAP, la centrale d'achats publics française, active dans de nombreux secteurs dont le secteur médical, a développé pour ses fournisseurs une solution appelée l'« affacturage collaboratif inversé ». La centrale d'achat public ne fournit pas de financement à ses fournisseurs, le mécanisme consiste à négocier le taux d'intérêt à payer par les fournisseurs à une institution financière pour ceux qui souhaitent recevoir un paiement anticipé et être payés dans un délai de deux jours en moyenne au lieu des 30 jours habituels.

Compte tenu de son pouvoir d'achat, les taux d'intérêt négociés par la centrale d'achats publics à la suite d'un appel d'offres ouvert sont beaucoup plus avantageux que les taux d'intérêt qu'un seul fournisseur pourrait obtenir sur le marché financier. La centrale d'achat public ne tire aucun avantage financier lorsque les fournisseurs utilisent ce mécanisme.

Les fournisseurs de l'UGAP peuvent opter pour cette solution sur la base du volontariat et l'utiliser pour toutes leurs factures ou au cas par cas, en fonction de leurs besoins financiers.

\section{Paiements contractuels instantanés en Corée}

L'agence coréenne des marchés publics apporte un soutien financier particulier aux PME impliquées dans les marchés publics. Les PME ont droit à un paiement initial allant jusqu'à $70 \%$ de la valeur d'un marché public. De plus, l'entreprise a droit à un paiement immédiat sur présentation de la facture pour certains marchés de biens, notamment les commandes sur des contrats à prix unitaire, les contrats à prix forfaitaires pour des biens d'une valeur maximale de 500 millions KRW (wons sud-coréens, environ 380000 EUR), ou à la demande de l'utilisateur final pour d'autres marchés de biens. Le paiement est alors effectué à partir d'un compte spécial de l'agence coréenne des marchés publics, puis il est remboursé par l'utilisateur final. Bien que les entités du gouvernement central aient droit à un délai de paiement pouvant aller jusqu'à cinq jours, le paiement immédiat permet aux PME d'être payées en quatre heures seulement.

Source : (OCDE, 2018[29]). 


\subsection{Mesures d'accompagnement pour une implémentation efficiente de politiques publiques dans les marchés publics}

L'utilisation des marchés publics comme un levier stratégique pour atteindre les OPP nécessite, en plus du développement et de la mise en place de stratégies et politiques, une analyse d'impact permettant d'évaluer dans quelle mesure les marchés publics contribuent à la réalisation des OPP. Une approche coordonnée avec les différentes parties prenantes et un système d'information efficace pour la collecte des données sont également nécessaires.

\subsubsection{Développer et mettre en place une méthodologie d'évaluation des OPP}

Les retombées de tout recours au système de passation de marchés publics au service d'OPP devraient être évaluées en fonction de critères adaptés afin d'offrir aux responsables publics les informations dont ils ont besoin quant aux avantages et aux coûts du dispositif. II faudrait mesurer l'efficacité au niveau de chaque marché, comme au niveau des résultats de l'ensemble du système de passation des marchés publics. Par ailleurs, l'utilisation des marchés publics comme levier stratégique au service d'OPP devrait être évaluée périodiquement pour éviter une possible surcharge d'objectifs (OCDE, 2015[17]). Cette étape est d'autant plus cruciale qu'elle est nécessaire pour réviser et mettre à jour les différentes stratégies ou politiques.

La plupart des gouvernements qui évaluent la mise en œuvre et les résultats du recours aux marchés publics comme levier pour atteindre des OPP ont fixé des objectifs quantitatifs relatifs à la valeur totale des dépenses consacrées aux biens et services durables. Une part plus réduite de gouvernements évaluent les résultats de ces politiques en termes de bénéfices tangibles pour l'environnement, l'économie et la société (ONU Environnement, 2017[6]). En effet, la mesure de l'efficacité des marchés publics pour atteindre des OPP est un défi pour plusieurs pays. Quelque $69 \%$ des pays de l'OCDE mesurent l'impact du recours aux marchés publics pour les politiques environnementales, respectivement $54 \%$ et $25 \%$ d'entre eux pour le développement des PME et l'innovation (graphique 5.8) (OCDE, 2016[37]).

\section{Graphique 5.8. Pourcentage des pays qui mesurent l'impact du recours aux marchés publics}
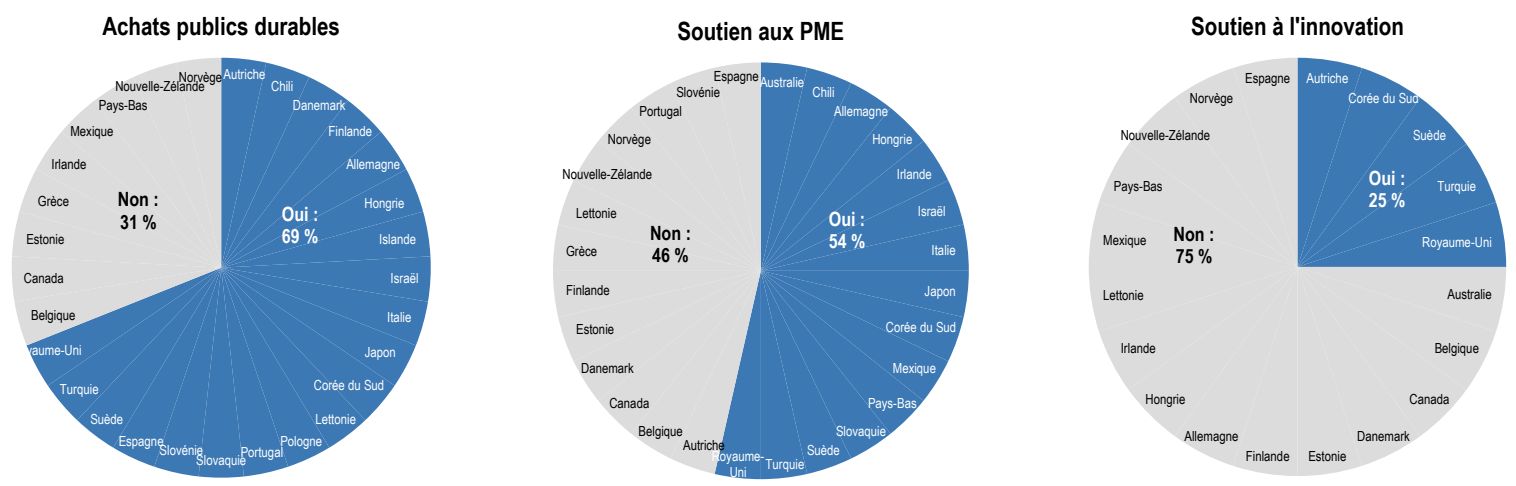

Source : (OCDE, 2016[37]).

L'évaluation de l'efficacité des marchés publics pour atteindre des OPP nécessite une approche cohérente et le suivi des étapes décrites dans l'encadré 5.9. En Algérie, aucune méthodologie d'étude d'impact n'a été mise en place, ce qui limite la possibilité d'analyser l'impact des politiques déjà en place. Ceci est dû à l'absence d'entité en charge de collecter les données (voir chapitre 1) et au manque d'indicateurs détaillés (voir chapitre 4), mais aussi de système de collecte de donnée (voir chapitre 3 ). Par exemple, aucune information n'est disponible sur l'efficacité des mesures de promotion de la production nationale, 
notamment la marge préférentielle de $25 \%$, ou sur la mise en place effective des dispositions relatives à la part des micro-entreprises dans les marchés publics, et ce, alors que le DMP stipule que les services contractants sont tenus de communiquer aux organismes chargés de la promotion des micro-entreprises toutes les informations concernant l'attribution et l'exécution des prestations mentionnées dans ces dispositions.

L'absence d'évaluation des politiques publiques limite la possibilité de revoir ou d'améliorer les éléments visant à prendre en compte les politiques publiques dans les marchés publics. Ceci est d'ailleurs souligné dans le Pacte national économique et social de la croissance qui mentionne que « la mise en place des outils de mesure de l'efficacité des politiques publiques est une condition sine qua non du succès du Pacte » (République algérienne démocratique et populaire, 2014[11]). Le gouvernement algérien devrait donc mettre en place une méthodologie d'étude d'impact avec des indicateurs clairs afin d'analyser le niveau d'implémentation et l'impact des politiques existante et à venir.

\section{Encadré 5.9. Les différentes étapes pour mesurer l'efficacité d'atteinte des OPP par les marchés publics}

- Développer des indicateurs détaillés en accord avec chacune des politiques envisagées.

- Assurer la collecte des données nécessaires.

- Développer des mesures incitatives afin de permettre de mesurer l'impact des politiques publiques et assurer des ressources financières suffisantes.

- Mettre en place des exigences réglementaires pour permettre de mesurer l'efficacité des marchés publics en matière d'atteinte des OPP (par exemple, il peut être demandé aux entités publiques de publier obligatoirement chaque année dans leur rapport d'activité, une section sur les avancements réalisés en termes de mise en place des politiques publiques).

- Évaluer les opportunités de consolidation ou rationalisation des politiques publiques qui peuvent se chevaucher.

- Utiliser les résultats de l'étude d'impact pour réviser et mettre à jour les stratégies ou politiques.

Source : (OCDE, 2016[38]).

Le développement de ces méthodologies d'étude d'impact doit être accompagné par la mise en place d'un système d'information et d'un système de passation électronique des marchés qui permet de collecter les données nécessaires à cette analyse. Comme décrit aux chapitres 3 et 4, l'Algérie ne dispose ni d'un système d'information ni d'un système de passation électronique des marchés, les données ne peuvent donc pas être collectées efficacement, et l'efficacité des dispositifs et des politiques ne peut être correctement évaluée. Le gouvernement algérien devrait donc mettre en place un système d'information qui permette de recueillir les données nécessaires à l'étude d'impact de la prise en compte des différentes politiques publiques dans les marchés publics.

\subsubsection{Encourager une culture des politiques publiques dans les marchés publics et renforcer les capacités des différentes parties prenantes}

Les marchés publics sont de plus en plus considérés comme une activité stratégique, et non plus seulement administrative, dont le but est de fournir une meilleure prestation de services publics aux citoyens. Faire des marchés publics un levier stratégique pour l'atteinte d'OPP passe nécessairement par la sensibilisation et le renforcement des capacités des différentes parties prenantes (OCDE, 2018[39]). 
Les acheteurs publics doivent intégrer de plus en plus d'OPP dans les procédures d'achat, en plus d'obtenir le meilleur rapport qualité/prix, et ce, dans les meilleurs délais. II est donc nécessaire de les sensibiliser à deux questions principales : i) pourquoi il est de la responsabilité de chaque acheteur de contribuer à la réalisation de ces objectifs ; et ii) comment intégrer les OPP dans les marchés publics. II faut mener des actions de renforcement des capacités des acheteurs publics dans ce domaine. En effet, la Recommandation de l'OCDE sur les marchés publics souligne la nécessité de prendre en compte la capacité des acheteurs publics à intégrer des OPP dans les marchés, ainsi que la charge associée au suivi de l'intégration de ces OPP (OCDE, 2015 $[17]$ ). II faut également renforcer les capacités du secteur privé en termes d'OPP, en particulier les PME. L'encadré 5.10 fournit des exemples de renforcement de capacité des acheteurs publics et du secteur privé pour améliorer la prise en compte des OPP dans les marchés publics.

\section{Encadré 5.10. Exemples de renforcement de capacité des acheteurs publics et du secteur privé pour améliorer la prise en compte des OPP dans les marchés publics}

\section{Pour les acheteurs publics (achats publics environnementaux) - République slovaque}

Le ministère de l'Environnement de la République slovaque a élaboré un plan national pour les achats publics environnementaux 2011-15, approuvé en janvier 2012.Pour atteindre les objectifs stratégiques de ce plan, il a été nécessaire d'impliquer aussi bien les organisations publiques que les municipalités dans un processus pédagogique. Leur implication a été encouragée par la mise en place d'actions de renforcement des capacités, comme par exemple la diffusion au plus grand nombre possible de parties prenantes d'informations techniques sur les achats écologiques et les enjeux connexes (eco-labels, systèmes de management environnemental, labels énergétiques, bureaux écologiques, etc.).

\section{Pour le secteur privé (développement des PME)}

\begin{tabular}{|c|c|}
\hline Pays & Types d'activités \\
\hline Australie & $\begin{array}{l}\text { Le gouvernement australien participe à des salons commerciaux et organise des événements } \\
\text { destinés à renforcer les capacités des fournisseurs. }\end{array}$ \\
\hline Autriche & $\begin{array}{l}\text { Les formations dispensées par les chambres économiques, les institutions centrales d'achat et les } \\
\text { cabinets d'avocats couvrent tous les aspects du système de passation des marchés publics. }\end{array}$ \\
\hline Canada & $\begin{array}{l}\text { Le Bureau des PME de SPAC (organisme central des achats du pays) expose durant des } \\
\text { événements et propose des séminaires et des webinaires à destination des PME. Exemples de } \\
\text { thèmes : faire des affaires avec le gouvernement canadien, obtenir des habilitations de sécurité, } \\
\text { comment fonctionnent les instruments d'achat spécifique (exemple : services professionnels). Un } \\
\text { catalogue des offres et l'inscription sont disponibles en ligne. }\end{array}$ \\
\hline Corée du Sud & $\begin{array}{l}\text { L'Institut de formation des achats publics - appartenant au Service des achats publics, l'organisme } \\
\text { central des achats du pays - organise des formations pour les fournisseurs, notamment sur les } \\
\text { contrats cadres, les politiques de management de la qualité, la détermination des prix contractuels } \\
\text { et les réclamations dans le secteur de la construction, ou encore comment s'inscrire et utiliser le } \\
\text { nouveau système d'e-procurement. En 2016, l'Institut a délivré des formations à } 2744 \text { fournisseurs. }\end{array}$ \\
\hline Hongrie & $\begin{array}{l}\text { Le bureau du Premier ministre et l'Autorité des marchés publics ont organisé plusieurs conférences } \\
\text { et ateliers de travail avec une importance particulière accordée aux nouvelles règles des marchés } \\
\text { publics. }\end{array}$ \\
\hline Norvège & $\begin{array}{l}\text { DIFI, l'organisme central des achats de la Norvège, a lancé un atelier de travail intitulé « Des achats } \\
\text { publiques équilibrés » dans lequel les PME sont formées aux processus de passation des marchés } \\
\text { publics. Ces ateliers de travail sont spécifiques, par secteur. }\end{array}$ \\
\hline Suisse & $\begin{array}{l}\text { Des événements d'information sont régulièrement organisés par les organismes centraux d'achats } \\
\text { afin d'accroître la connaissance des opportunités de soumissionner pour les achats du } \\
\text { gouvernement, les types de procédures et les domaines d'achats (fournitures, services, travaux). }\end{array}$ \\
\hline
\end{tabular}

Source : (OCDE, 2018[39] ; OCDE, 2018[29]). 
En Algérie, des actions de sensibilisation ou de renforcement des capacités dédiées à l'utilisation des marchés publics comme levier stratégique pour l'atteinte d'OPP n'ont pas été mises en place pour les acheteurs publics comme pour le secteur privé, et ce, alors que ces actions pourraient être menées en marge d'événements ou d'activités déjà en place. Ainsi, par exemple, l'Inapi organise tous les ans le salon national de l'innovation, mais aucun événement en marge de ce salon n'est spécifiquement dédié aux marchés publics. De plus, les formations proposées par l'Institut supérieur de gestion et de planification (ISGP) n'intègrent pas de modules dédiés à l'utilisation des marchés publics comme levier stratégique pour atteindre des OPP. Le gouvernement algérien devrait donc mener des actions de sensibilisation et de renforcement des capacités dans ce domaine. Ces actions devront cibler les agents en charge des marchés publics, le secteur privé, mais aussi les corps de contrôle. En effet, la sensibilisation des corps de contrôle et le renforcement de leurs capacités est un élément primordial à prendre en compte afin d'assurer une compréhension commune de la prise en compte des OPP dans les marchés publics et donc limiter le risque de blocage de la procédure. 


\section{Propositions d'action}

\begin{tabular}{|c|c|c|c|}
\hline Recommandations & $\begin{array}{l}\text { Court } \\
\text { terme }\end{array}$ & $\begin{array}{c}\text { Moyen } \\
\text { terme }\end{array}$ & $\begin{array}{l}\text { Long } \\
\text { terme }\end{array}$ \\
\hline Assurer une cohérence entre les différents OPP et le cadre réglementaire régissant les marchés publics & & $\mathrm{X}$ & \\
\hline $\begin{array}{l}\text { Développer des stratégies identifiant clairement les marchés publics comme un levier stratégique pour la } \\
\text { mise en place des différents OPP : développement durable, développement des PME, innovation et } \\
\text { responsabilité sociétale des entreprises }\end{array}$ & & $\mathrm{X}$ & \\
\hline $\begin{array}{l}\text { Assurer l'engagement et la participation des différentes parties prenantes à l'élaboration d'une stratégie } \\
\text { visant à utiliser les marchés publics comme un levier stratégique pour l'atteinte d'OPP. }\end{array}$ & & $\mathrm{X}$ & \\
\hline Mettre en place l'ARMP & $\mathrm{X}$ & & \\
\hline $\begin{array}{l}\text { Prévoir des objectifs clairs de politiques publiques à atteindre dans le cadre des stratégies à développer } \\
\text { pour l'utilisation des marchés publics comme un levier stratégique. }\end{array}$ & & & $\mathrm{X}$ \\
\hline $\begin{array}{l}\text { Renforcer les capacités des acheteurs publics à utiliser les critères de sélection et d'attribution et mes } \\
\text { clauses d'exécution de marchés prenant en compte les enjeux sociaux et environnementaux }\end{array}$ & & $\mathrm{X}$ & \\
\hline $\begin{array}{l}\text { Développer des lignes directrices pour l'utilisation de critères de sélection et d'attribution et des clauses } \\
\text { d'exécution de marchés prenant en compte les enjeux sociaux et environnementaux }\end{array}$ & $\mathrm{X}$ & & \\
\hline Mettre en place un système d'écolabels, qui pourraient être octroyés par I'IANOR & & & $X$ \\
\hline Harmoniser le vocabulaire utilisé dans les différents cadres réglementaires concernant les PME & & & $\mathrm{X}$ \\
\hline $\begin{array}{l}\text { Réaliser une étude pour évaluer l'impact de l'article } 87 \text { ( } 20 \% \text { de la commande publique réservée aux PME) } \\
\text { sur le développement des PME }\end{array}$ & & $\mathrm{X}$ & \\
\hline $\begin{array}{l}\text { Imposer l'allotissement de tous les marchés tout en prévoyant une justification détaillée de la part des } \\
\text { entités publiques lorsque l'allotissement n'est pas possible }\end{array}$ & & & $\mathrm{X}$ \\
\hline $\begin{array}{l}\text { Renforcer les contrôles pour les procédures en dessous des seuils afin de s'assurer que les besoins ne } \\
\text { soient pas fractionnés en vue d'échapper aux procédures formalisées }\end{array}$ & & $\mathrm{X}$ & \\
\hline Promouvoir la cotraitance auprès du secteur privé & $\mathrm{X}$ & & \\
\hline $\begin{array}{l}\text { Créer un espace ou un outil visant à faciliter le contact entre les entreprises désireuses de former un } \\
\text { groupement afin de répondre à un appel d'offres donné }\end{array}$ & & & $\mathrm{X}$ \\
\hline Autoriser la sous-traitance pour les marchés de fournitures & & & $X$ \\
\hline $\begin{array}{l}\text { Développer des lignes directrices pour aider les entités publiques à choisir des critères de qualification et } \\
\text { des exigences financières proportionnels à l'objet du marché afin de ne pas nuire à l'accès des PME aux } \\
\text { marchés publics }\end{array}$ & & $\mathrm{X}$ & \\
\hline $\begin{array}{l}\text { Dispenser toutes les PME de la présentation d'une caution pour la soumission ou bien diminuer leur } \\
\text { pourcentage pour les PME }\end{array}$ & & & $\mathrm{X}$ \\
\hline S'assurer du respect des délais de paiement et mettre en place des dispositifs pour les réduire & & $\mathrm{X}$ & \\
\hline $\begin{array}{l}\text { Développer et mettre en place une méthodologie d'évaluation des objectifs de politique publiques (celles } \\
\text { existantes et celles à venir) }\end{array}$ & & & $X$ \\
\hline $\begin{array}{l}\text { Mettre en place un système d'information qui permette de recueillir les données nécessaires à l'étude } \\
\text { d'impact et à la prise en compte des différentes politiques publiques dans les marchés publics. }\end{array}$ & & & $\mathrm{X}$ \\
\hline $\begin{array}{l}\text { Renforcer les capacités des acteurs du système de passation des marchés publics (acheteurs publics, } \\
\text { secteur privé, corps de contrôle) en matière de politiques publiques }\end{array}$ & & $\mathrm{X}$ & \\
\hline
\end{tabular}

Note : Court terme : recommandation à mettre en application dans les 12 mois suivants ;

Moyen terme : recommandation à mettre en application dans les $1-2$ ans ;

Long terme : recommandation à mettre en application dans plus de 2 ans. 


\section{Références}

Commission Européenne (2011), Study on "Strategic use of public procurement in promoting green, social and innovation policies », https://publications.europa.eu/en/publication-detail//publication/6a5a4873-b542-11e7-837e-01aa75ed71a1/language-en/format-PDF (consulté le 13 novembre 2018).

Direction des achats de l'État (DAE) (s.d.), Un nouveau service sur la Place pour les PME : la bourse à la cotraitance, https://www.economie.gouv.fr/dae/nouveau-service-sur-place-pourpme-bourse-a-cotraitance-0 (consulté le 14 novembre 2018).

Eccles, I. (2012), « The Impact of a Corporate Culture of Sustainability on Corporate Behavior and Performance ", n 12-035, http://trippel.sdg.no/wp-content/uploads/2014/09/EcclesHBR The-Impact-of-a-Corporate-Culture-of-Sustainability1.pdf (consulté le 15 novembre 2018).

GII (2018), Indicator Rankings \&amp; Analysis | Global Innovation Index, https://www.globalinnovationindex.org/analysis-indicator (consulté le 8 novembre 2018).

Gouvernement du Canada (2018), Stratégie ministérielle de développement durable 2017 à 2020 de Services publics et Approvisionnement Canada, https://www.tpsgcpwgsc.gc.ca/rapports-reports/smdd-dsds/index-fra.html (consulté le 12 novembre 2018).

Ministère de l'Industrie et des Mines, Algérie (s.d.), L'Institut algérien de normalisation, http://www.mdipi.gov.dz/?L-Institut-Algerien-de (consulté le 9 novembre 2018).

Ministère de l'Industrie et des Mines, Algérie (s.d.), Organisation de la Direction Générale de la Compétitivité Industrielle (DGCl), http://www.mdipi.gov.dz/?-Direction-Generale-de-la(consulté le 22 octobre 2018).

Ministère de la Transition écologique et solidaire, France (2018), Les achats publics durables, https://www.ecologique-solidaire.gouv.fr/achats-publics-durables (consulté le 12 novembre 2018).

Nations Unies (2002), Rapport du Sommet mondial pour le développement durable, https://www.francophonie.org/IMG/pdf/Johannesburg.pdf (consulté le 14 mai 2019).

OCDE (2019), Améliorer l'accès des PME aux marchés publics en Tunisie: la voie à suivre, OCDE, Paris, http://www.oecd.org/countries/tunisia/ameliorer-acces-PME-aux-marchespublics-Tunisie.pdf (consulté le 11 juin 2019).

OCDE (2018), Procurement Review of the Chamber of Commerce of Bogotá, Colombia: Aligning Practices with the OECD Public Procurement Recommendation, OCDE, Paris, https://www.oecd.org/gov/public-procurement/publications/procurement-review-chambercommerce-bogota.pdf.

OCDE (2018), « Responsible Business Conduct in Public Procurement ».

OCDE (2018), SMEs in Public Procurement : Practices and Strategies for Shared Benefits, Examens de l'OCDE sur la gouvernance publique, Éditions OCDE, Paris, https://dx.doi.org/10.1787/9789264307476-en. 
OCDE (2017), Public Procurement for Innovation: Good Practices and Strategies, Examens de I'OCDE sur la gouvernance publique, Éditions OCDE, Paris, http://dx.doi.org/10.1787/9789264265820-en.

OCDE (2017), Small, Medium, Strong. Trends in SME Performance and Business Conditions, Éditions OCDE, Paris, http://dx.doi.org/10.1787/9789264275683-en.

OCDE (2016), Checklist for Supporting the Implementation of OECD Recommendation of the Council on Public Procurement: Balance, OCDE, Paris, http://www.oecd.org/governance/procurement/toolbox/search/Checklist $\% 2004 \% 20$ Balance.pd f (consulté le 13 décembre 2018).

OCDE (2016), « Réponses au questionnaire de l'OCDE sur les marchés publics ».

OCDE (2015), Going Green: Best Practices for Sustainable Procurement, OCDE, Paris, https://www.oecd.org/governance/ethics/Going Green Best Practices for Sustainable Proc urement.pdf (consulté le 10 octobre 2018).

OCDE (2015), Recommandation du Conseil de l'OCDE sur les marchés publics, OCDE, Paris, http://www.oecd.org/fr/gov/ethique/Recommandation-OCDE-sur-les-marches-publics.pdf (consulté le 16 août 2018).

OCDE (2012), Recommandation du conseil de l'OCDE sur la lutte contre les soumissions concertées dans les marchés publics, OCDE, Paris, http://www.oecd.org (consulté le 19 juillet 2018).

OCDE (2011), Competition and Procurement: Key findings, OCDE, Paris, http://www.oecd.org/daf/competition/sectors/48315205.pdf (consulté le 14 novembre 2018).

OCDE (2011), Les principes directeurs de l'OCDE à l'intention des entreprises multinationales, Éditions OCDE, Paris, https://dx.doi.org/10.1787/9789264115439-fr.

OCDE (2011), "Setting the Award Criteria », SIGMA Public Procurement Briefs, $\mathrm{n}^{\circ}$ 8, Éditions OCDE, Paris, https://dx.doi.org/10.1787/5js4wzvcz69q-en.

OCDE (à paraître), Examen d'intégrité dans les marchés publics du Québec : Une approche stratégique contre les risques de corruption.

OCDE/SIGMA (2016), « Brief 33, Small and Medium-sized entreprises (SMEs) in Public Procurement », http://www.sigmaweb.org/publications/Public-Procurement-Policy-Brief-33200117.pdf (consulté le 10 novembre 2017).

OCDE/SIGMA (2016), Brief 36, Division of Contracts into Lots, http://www.oecd.org/officialdocuments/publicdisplaydocumentpdf/?cote=daf/comp/wp2 (consulté le 28 février 2019).

ONU Environnement (2017), Panorama Mondial des Achats Publics Durables, http://www.scpclearinghouse.org/resource/sustainable-public-procurement-global-review$\underline{2013}$ (consulté le 18 octobre 2018).

ORSE (s.d.), RSE Algerie : Responsabilité sociétale des entreprises en Algérie, http://www.rsealgerie.org/fr/ (consulté le 14 novembre 2018). 
PNUD (2018), Objectifs de développement durable, http://www.undp.org/content/undp/fr/home/sustainable-development-goals/ (consulté le 18 octobre 2018 ).

Programme P3A (2018), Revue de presse -P3A: " Appui au Ministère de l'Industrie et des Mines dans sa stratégie d'innovation industrielle », http://www.p3a-algerie.org/wpcontent/uploads/2018/10/Revue de presse -cloture MIM.pdf (consulté le 8 novembre 2018).

République algérienne (2017), « Loi n 17-02 du 10 janvier 2017 portant loi d'orientation sur le développement de la petite et moyenne entreprise (PME). », https://www.droitafrique.com/uploads/Algerie-Loi-2017-02-orientation-developpement-pme.pdf (consulté le 25 octobre 2018).

République algérienne démocratique et populaire (2014), Pacte national économique et social de croissance, http://www.fce.dz/wp-content/uploads/2015/09/pacte-national-economique-etsocial-de-croissance.pdf (consulté le 19 octobre 2018).

Service Public de Wallonie (2017), Plan d'actions achats publics responsables 2017-2019, http://developpementdurable.wallonie.be/sites/default/files/2017-10/plan apr complet.pdf (consulté le 12 novembre 2018).

U.S. Department of State (2018), Investment Climate Statements for 2018- Algeria, https://www.state.gov/e/eb/rls/othr/ics/investmentclimatestatements/index.htm?year=2018\&dli $\mathrm{d}=281653 \#$ wrapper (consulté le 25 février 2019).

UNEP (2015), The Sustainable Public Procurement Programme of the 10-Year Framework of Programmes (10YFP SPP Programme) Principles of Sustainable Public Procurement, http://www.unep.org/10yfp/procurement! (consulté le 14 mai 2019).

Union Européenne (2016), Buying green! A Handbook on Green Public Procurement, http://dx.doi.org/10.2779/246106.

Union Européenne (2014), Directive 2014/24/UE sur la passation des marchés publics, https://eur-lex.europa.eu/legal-content/FR/TXT/PDF/?uri=CELEX:32014L0024\&from=SL (consulté le 8 mars 2019).

Union Européenne (s.d.), Règles et procédures en matière de marchés publics en Europe, https://europa.eu/youreurope/business/public-tenders/rules-procedures/index fr.htm (consulté le 12 novembre 2018).

Yukins, C. et G. Piga (2012), « Dialogue: Set-asides for small and medium firms, as in the USA system with the Small Business Act that reserves shares of tenders to SMEs only », dans Piga, G. et S. Treumer (dir. pub.), The Applied Law and Economics of Public Procurement, Routledge, https://www.routledge.com/The-Applied-Law-and-Economics-of-PublicProcurement-1st-Edition/Piga-Treumer/p/book/9780415622165 (consulté le 25 octobre 2018). 

Le renforcement des capacités, un prérequis au bon fonctionnement du système de passation des marchés publics en Algérie

Le renforcement des capacités est un défi majeur pour l'Algérie afin d'assurer l'efficience de son système de passation des marchés publics et la mise en place effective des réformes dans ce domaine. Ce chapitre analyse le système en place pour assurer des capacités suffisantes et adéquates des parties prenantes au système de passation des marchés publics. Par ailleurs, il fournit des recommandations et identifie des actions concrètes afin de renforcer les capacités des acteurs du système et d'assurer la professionnalisation de la fonction achat.

Les données statistiques concernant Israël sont fournies par et sous la responsabilité des autorités israéliennes compétentes. L'utilisation de ces données par l'OCDE est sans préjudice du statut des hauteurs du Golan, de Jérusalem Est et des colonies de peuplement israéliennes en Cisjordanie aux termes du droit international. 
Un système de passation des marchés publics dont les acteurs disposent des capacités adéquates est un élément clé pour le bon fonctionnement du système ainsi que pour le succès de toute réforme dans ce domaine. En effet, les marchés publics représentent une part importante de la dépense publique : environ $12 \%$ du PIB dans les membres pays de l'OCDE et $22 \%$ du PIB en Algérie. La bonne gestion des marchés publics dépend en grande partie de la capacité des différents acteurs et parties prenantes en nombre et en termes de compétences et de l'adéquation de ces dernières avec les exigences professionnelles que requiert un système de passation des marchés publics efficient.

L'expérience de l'OCDE dans l'analyse de systèmes de passation des marchés publics démontre que disposer d'agents avec les capacités adéquates est l'un des cinq piliers d'un système de passation des marchés publics efficace, du fait de son impact et son lien avec les autres principes d'un système efficient (voir encadré 6.1) (OCDE, 2016[1]), (OCDE, 2017[2]).

\section{Encadré 6.1.Principaux liens entre la capacité et d'autres principes de la Recommandation du Conseil de l'OCDE sur les marchés publics}

Si le principe de capacité est lié à tous les principes de la Recommandation du Conseil de l'OCDE sur les marchés publics, il est particulièrement associé aux principes suivants :

- Intégrité : des agents chargés de la passation des marchés publics disposant de capacités adéquates sont mieux à même de se conformer aux procédures et d'assurer leur mise en œuvre permettant ainsi de prévenir les risques de corruption.

- Accès : des agents chargés de la passation des marchés publics disposant de capacités adéquates assurent une documentation d'appel d'offres claire et cohérente, si possible standardisée.

- Mise en balance : des agents chargés de la passation des marchés publics disposant de capacités adéquates facilite la réalisation d'objectifs complémentaire des politiques publiques.

- Efficacité : des agents chargés de la passation des marchés publics disposant de capacités adéquates assurent l'optimisation des ressources.

- Systèmes électroniques de passation des marchés publics : des agents chargés de la passation des marchés publics et des soumissionnaires et fournisseurs disposant de capacités adéquates sont en mesure d'utiliser un système de passation électronique des marchés publics.

- Évaluation : la désignation d'équipes ou d'unités spécifiques pour l'évaluation des informations relatives aux marchés publics et la performance du système de passation des marchés publics permet d'améliorer les capacités dans le domaine des marchés publics.

- Gestion de risques : des agents chargés de la passation des marchés publics disposant de capacités adéquates connaissent, comprennent et mettent en œuvre les stratégies de gestion des risques, leur implémentation et les mesures mises en place pour gérer les risques.

- Responsabilité : le contrôle interne et les contrôles externes doivent être coordonnés, dotés de ressources adéquates et intégrées afin d'être efficaces.

Source : (OCDE, 2016[3]).

Conscients du rôle central de la capacité des différentes parties prenantes et en particulier des agents en charge de la passation des marchés publics dans l'amélioration du fonctionnement du système dans son ensemble, de nombreux pays ont décidé d'investir dans le renforcement de ces capacités afin de pouvoir fournir aux citoyens des infrastructures et des services correspondant à leur besoin, au meilleur rapport 
qualité prix et dans les délais. Pour ce faire, l'OCDE recommande aux pays de : i) veiller à ce que les agents chargés de la passation des marchés publics satisfassent à des exigences professionnelles élevées en termes de connaissances, de savoir-faire et d'intégrité en disposant d'un ensemble d'outils spécifiques et récents, avec, par exemple la reconnaissance de la passation des marchés publics en tant que profession spécifique ; ii) offrir aux agents en charge de la passation des marchés publics des possibilités d'évolution de carrière attractives, compétitives et fondées sur le mérite ; et iii) favoriser la collaboration avec les centres de savoir tels que les universités, les laboratoires d'idées ou les centres stratégiques afin d'améliorer les compétences des agents en charge de la passation des marchés publics. (OCDE, 2015[4])

L'Algérie a adopté en 2015 le décret 15-247, portant réglementation des marchés publics et des délégations de service public (DMP), mettant ainsi à jour son cadre réglementaire conformément aux bonnes pratiques internationales. Cependant, les capacités des différents acteurs du système de passation des marchés publics ont été identifiées, notamment par les organes de contrôle tels que I'Inspection générale des finances (IGF), comme un obstacle majeur à l'application effective du DMP et plus largement au bon fonctionnement de l'ensemble du système.

Ce chapitre analyse les lacunes du système de passation des marchés publics en termes de capacités et fournit des recommandations conformes aux meilleures pratiques internationales. En particulier, ce chapitre traite de la nécessité de développer une stratégie nationale de renforcement des capacités.

\subsection{Pour le développement d'une stratégie nationale de renforcement des capacités dans le domaine des marchés publics}

Des études de l'OCDE ont montré que la principale fragilité des systèmes de passation des marchés publics réside dans le niveau de capacité des acteurs impliqués dans le cycle de passation des marchés publics (OCDE, 2017 $[5]$ ). Les solutions pour y remédier sont nombreuses et leurs mises en œuvre doivent être définies sur mesure et coordonnées, ce qui implique le développement d'une stratégie nationale afin de renforcer le système de passation des marchés publics dans son ensemble. Le développement de cette stratégie permet d'adapter les objectifs au contexte du pays concerné, de les prioriser, de les planifier, de mesurer l'avancement de leur implémentation et d'évaluer les résultats. Le développement d'une stratégie nationale constitue donc une étape majeure qui suscite l'intérêt et l'implication de l'ensemble des parties prenantes du système, et ce, au niveau central comme au niveau local, et requiert une approche méthodologique basée sur 4 étapes principales :i) l'analyse de l'existant; ii) le développement d'une stratégie ; iii) la préparation pour sa mise en place effective ; et iv) le réexamen de la situation (comme indiqué au chapitre 3).

L'application effective de cette approche méthodologique nécessite tout d'abord la désignation d'une autorité responsable de la stratégie, ainsi que d'un comité de pilotage et l'identification des parties prenantes du système de passation des marchés publics et de leurs besoins (OCDE, 2016[6] $)$.

\subsubsection{Une approche collaborative et inclusive pour le développement de la stratégie de renforcement des capacités dans le domaine des marchés public}

\section{Le rôle central de l'Autorité de régulation des marchés publics (ARMP) dans le développement de la stratégie nationale de renforcement des capacités dans le domaine des marchés publics}

Afin de renforcer un système de passation des marchés publics dans son ensemble, il est nécessaire de développer une stratégie nationale de renforcement des capacités. L'élaboration de cette stratégie, le suivi de son implémentation, l'évaluation des progrès et des résultats obtenus doivent être confiés à une entité 
publique qui sera chargée du pilotage de cette stratégie. Cette entité doit mener ses missions en collaboration avec l'ensemble des parties prenantes et peut être, selon les pays, un ministère ou l'entité en charge du système de passation des marchés publics. (OCDE, 2016[6])

Les responsabilités de l'entité chargée du pilotage d'une stratégie nationale de renforcement des capacités dans les marchés publics sont décrites dans l'encadré 6.2. Par exemple, en France, c'est la Direction des achats de l'État qui a pour mission de définir la stratégie de formation des acteurs de la fonction achat de l'État, de piloter l'offre de formation et de proposer des mesures permettant de renforcer la professionnalisation des acteurs de la fonction achat. Elle a également comme prérogative de structurer les parcours professionnels des acteurs de la fonction achat dans le cadre d'une filière d'achats commune aux services de l'État et aux établissements publics (Direction des achats de l'État (DAE), 2018[7]). Cette pratique est également courante dans la région Moyen-Orient et Afrique du Nord (MENA),en Tunisie par exemple, cette mission est confiée à l'Observatoire national des marchés publics, un organe de la Haute instance de la commande publique chargé d'assister les acheteurs publics par des programmes de formation, des documents types, des applications informatiques et des mesures d'aide diverses en vue de faciliter et simplifier leurs fonctions (Gouvernement de Tunisie, 2018[8]).

\section{Encadré 6.2. Exemples de responsabilités de l'entité de pilotage d'une stratégie nationale de renforcement des capacités dans les marchés publics}

- Coordonner le programme de renforcement des capacités liées aux marchés publics.

- Mener les évaluations des besoins et intégrer les résultats dans la stratégie finale.

- Identifier et prioriser les objectifs de la stratégie.

- Élaborer le plan d'actions (un atelier de travail pourrait être nécessaire pour démarrer les discussions).

- Coordonner avec le ministère en charge de la fonction publique la prise en compte des marchés publics dans la formation des fonctionnaires.

- Si applicable, réviser le cadre régissant la fonction publique pour y intégrer des dispositions relatives notamment: aux carrières dans le domaine des marchés publics, à l'engagement du fonctionnaire à travailler dans la fonction publique, à l'élaboration d'un code de déontologie et manuel de travail, aux mesures incitatives pour ces agents.

- Si applicable, coordonner avec le ministère de l'Éducation les dispositions nécessaires pour réglementer le recrutement, les diplômes et les certifications, ainsi que les accords avec les institutions de formation. La participation du ministère de l'Éducation supérieure sera également requise pour assurer l'incorporation des marchés publics dans le cursus universitaire.

- Identifier les sources potentielles de financement et coordonner avec les bailleurs de fonds potentiels afin d'éviter les doublons.

- Suivre les accréditations de formation et superviser la diffusion de la formation.

- Contrôler les résultats selon les prescriptions établies par l'autorité de pilotage (résultats par type d'entité adjudicatrice [locale, nationale, ministère], par secteurs, etc.).

- Dresser une liste d'enseignements tirés.

- Adapter et réviser le plan d'action autant que nécessaire.

Source : (OCDE, 2016[6]). 
En Algérie, d'après l'article 213 du DMP, ces missions sont confiées à l'ARMP, placée sous l'autorité du ministère des Finances, et ayant notamment comme attributions d'informer, de diffuser et de vulgariser tous documents et informations relatifs aux marchés publics et aux délégations de service public, ainsi que d'initier les programmes de formation et de promouvoir la formation en marchés publics et en délégations de service public. Au vu de ses attributions, l'ARMP devrait être en charge du développement d'une stratégie nationale de renforcement des capacités dans les marchés publics. Cependant, malgré l'entrée en vigueur du DMP en décembre 2015, l'ARMP n'est toujours pas mise en place. L'Algérie verrait son système de passation des marchés publics renforcé en mettant en place l'ARMP et en lui confiant le pilotage de la stratégie nationale de renforcement des capacités dans le domaine des marchés publics.

\section{Un cadre réglementaire stable est nécessaire pour le développement des actions de renforcement des capacités}

En plus d'un cadre institutionnel clair, le développement d'une stratégie de renforcement des capacités requiert un cadre réglementaire clair, stable et cohérent. En effet, comme décrit dans le chapitre 1, ces cadres sont essentiels pour élargir la participation aux marchés publics et constituent des points de départ cruciaux pour assurer la viabilité et l'efficience des systèmes de passation de marchés publics (OCDE, 2015[4]). Un cadre réglementaire en constante évolution, ou dont toutes les dispositions ne sont pas appliquées, ne permet pas de mener des actions de renforcement des capacités durables et claires.

En Algérie, toutes les dispositions du DMP ne sont pas appliquées, ce qui entrave le bon fonctionnement du système de passation des marchés publics, mais également le développement d'une stratégie nationale de renforcement des capacités dans le domaine des marchés publics. L'Algérie devrait donc s'assurer de l'application et la mise en œuvre de l'ensemble des dispositions du DMP et autres textes réglementaires pour développer une stratégie de renforcement des capacités.

\section{Le développement de la stratégie nationale de renforcement des capacités dans les marchés publics doit être mené en collaboration avec d'autres parties prenantes}

Le développement de la stratégie nationale de renforcement des capacités sur les marchés publics doit être mené par une entité de pilotage préalablement identifiée, en collaboration avec un ensemble de parties prenantes pertinentes regroupées dans un comité de pilotage. Ce comité peut être constitué notamment d'experts du système de passation des marchés publics, de membres des commissions des marchés ou d'acheteurs publics expérimentés de différents ministères et entités publiques, des corps de contrôle et des instituts de formation. Cette collaboration doit inclure des fonctionnaires issus d'entités centrales, mais également régionales et locales, afin d'être représentative de l'ensemble du pays et ainsi accroître la responsabilisation des parties impliquées dans son implémentation (OCDE, 2016[6]).

En Algérie, différentes entités peuvent être identifiées comme parties prenantes au développement de la stratégie nationale de renforcement des capacités dans les marchés publics (graphique 6.1). 
Graphique 6.1. Les parties prenantes au développement de la stratégie nationale de renforcement des capacités dans les marchés publics en Algérie

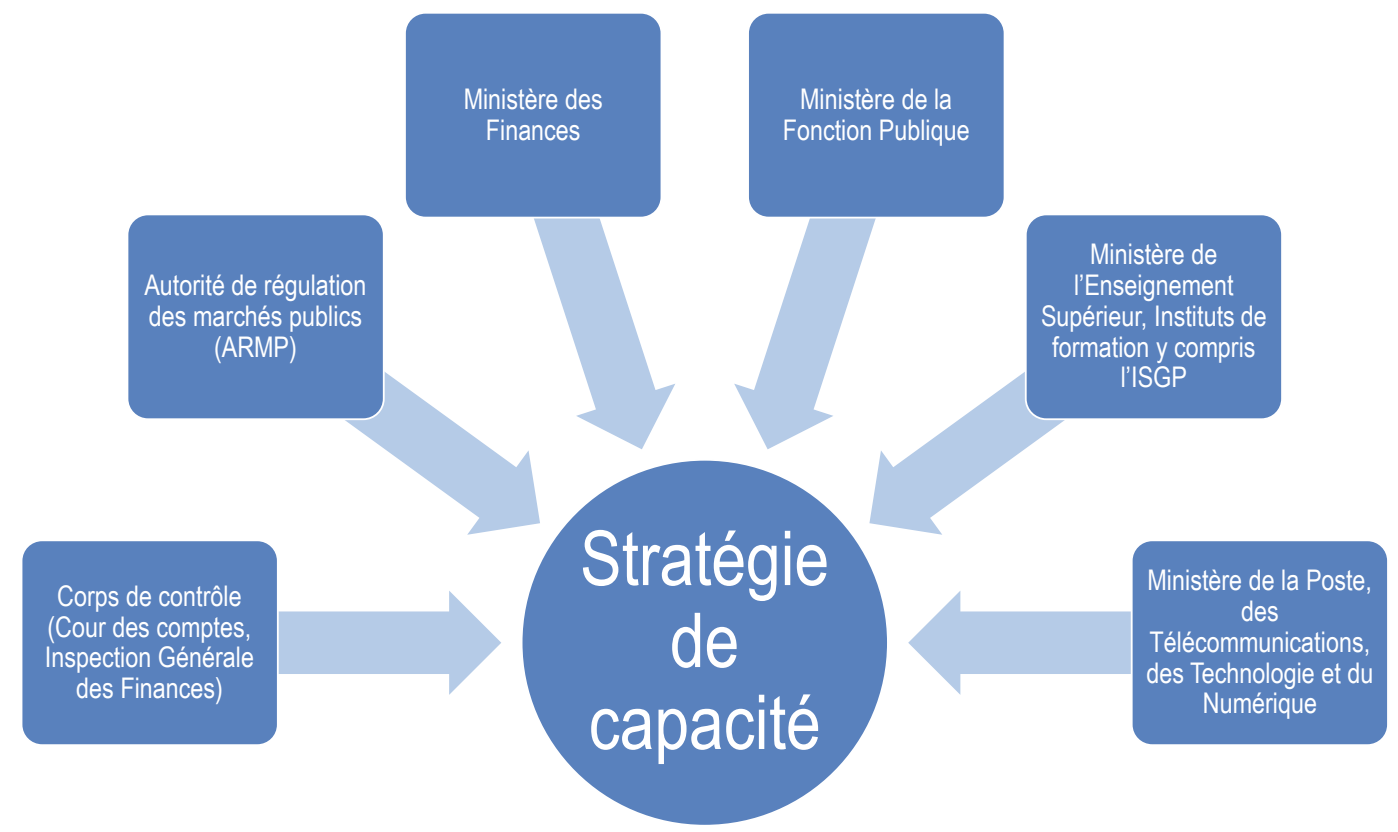

L'ARMP devrait être l'entité de pilotage de la stratégie de renforcement des capacités dans le domaine des marchés publics. L'implication du ministère de la Fonction publique est nécessaire car la stratégie peut prévoir des éléments relevant de sa compétence, tels que les formations dans la fonction publique ou la modification du statut de la fonction publique en prévoyant des dispositions particulières pour les agents en charge de la passation des marchés publics (salaires, évolution de carrière).

Le ministère de l'Enseignement supérieur doit, quant à lui, assurer que des cursus universitaires dans le domaine des marchés publics soient disponibles ; il pourra servir de lien avec d'autres organismes de formation, comme mentionné à la section 6.2.3.

Les corps de contrôle pourront être des sources d'informations essentielles sur les erreurs récurrentes et les dysfonctionnements constatés du système de passation des marchés publics et ainsi veiller à la correction des failles constatées.

Le ministère de la Poste, des Télécommunications, des Technologies et du Numérique (MPTTN) pourra également être associé afin de développer les capacités nécessaires à la mise en place du système électronique de passation des marchés publics pour le secteur public comme pour le secteur privé.

Les instituts de formation dans le domaine des marchés publics, y compris l'Institut supérieur de gestion et de planification (ISGP), devraient également être impliqués dans le développement de la stratégie nationale étant donné leur expérience dans le domaine et leur maîtrise du sujet.

L'Algérie devrait mettre en place un comité de pilotage, dont la supervision serait confiée à l'ARMP ou toute autre entité ayant ses prérogatives, afin de développer une stratégie nationale de renforcement des capacités dans le domaine des marchés publics grâce à un travail collaboratif de l'ensemble des parties prenantes qui peuvent jouer un rôle clé dans ce domaine. En attendant la mise en place de l'ARMP, la Division des marchés publics peut prendre en charge cette mission, sous réserve d'un certain nombre d'aménagements réglementaires (voir chapitre 1). 


\subsubsection{Identifier l'ensemble des parties prenantes impliquées dans le cycle de passation des marchés publics pour renforcer leurs capacités}

\section{L'identification et le recensement des agents chargés des marchés publics et des autres acteurs du système}

Une stratégie de renforcement des capacités dans le domaine des marchés publics permet de renforcer l'efficacité de l'ensemble du système. En effet, en plus des agents chargés de la passation des marchés publics, d'autres acteurs jouent un rôle majeur dans la passation, la gestion et le contrôle des activités de marchés publics.

La Recommandation du Conseil de l'OCDE sur les marchés publics indique qu'un système de passation des marchés publics efficace doit se doter des effectifs suffisants en nombre et en compétences, (OCDE, $2015_{[4]}$ ). Cela suppose une identification préalable des agents en charge des marchés publics, mais aussi de l'ensemble des acteurs intervenants tout au long du cycle de passation des marchés publics et de leurs capacités (OCDE, 2016[6]). En Algérie, plusieurs parties prenantes jouent un rôle central dans le bon fonctionnement du système de passation des marchés publics (voir graphique 6.2).

\section{Graphique 6.2. Les acteurs du système de passation des marchés publics en Algérie}

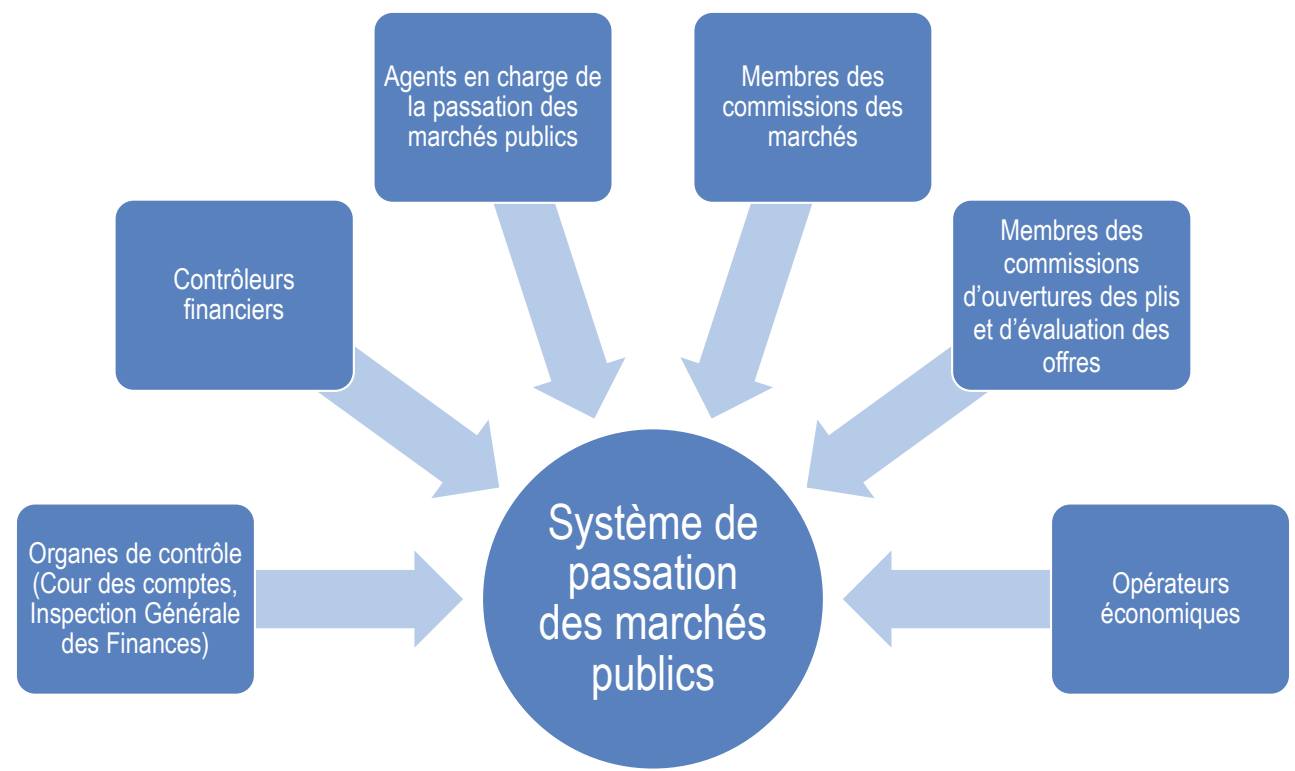

Le système de passation des marchés publics algérien implique les agents chargés de la passation des marchés, notamment ceux chargés de la rédaction des spécifications techniques, de la rédaction des documents d'appel d'offres et du suivi d'exécution du marché.

II n'existe cependant pas de données relatives au nombre total d'agents impliqués dans le système de passation des marchés publics algérien. Ceci peut s'expliquer par le fait que le métier d'acheteur n'est pas reconnu comme un métier à part entière (voir section 6.2.1). Cependant, l'un des prérequis au développement d'une stratégie de renforcement des capacités dans les marchés publics est l'identification de la population cible. Certains pays, comme par exemple le Pérou, ont procédé à un recensement des agents chargés de la passation des marchés publics dans l'ensemble des entités publiques au niveau national, régional et local (voir encadré 6.3). Pour le développement de sa stratégie nationale de renforcement des capacités dans les marchés publics, le gouvernement algérien devrait procéder à l'identification et au recensement de l'ensemble des agents publics en charge de la passation des marchés 
publics, tant au niveau central qu'au niveau régional et local. L'identification et le recensement devront être pilotés par l'ARMP et des délégations de service public afin de pouvoir mener à bien ses missions.

\section{Encadré 6.3. Le recensement du personnel chargé de la passation des marchés publics au} Pérou

Les politiques relatives à la fonction publique nationale du Pérou sont coordonnées par l'autorité centrale des ressources humaines, l'Autorité nationale de la fonction publique (SERVIR). Cette instance, sous l'autorité de la Présidence du conseil des ministres, a pour rôle la planification et la formulation de politiques publiques et normes techniques en relation avec le système de gestion du personnel administratif ; le développement, la gestion et l'évaluation des politiques de formation ; et l'organisation et la supervision des concours pour le recrutement du personnel.

Entre 2010 et 2014, SERVIR a procédé au recensement des agents publics en charge de la passation des marchés publics, au niveau national, régional et municipal. Ce recensement a permis d'identifier plus de 6000 agents répartis dans plus de 1500 entités.

Source : Adapté de (OCDE, 2017[5]).

En plus des agents en charge de la passation des marchés publics, d'autres acteurs publics sont impliqués dans le cycle de passation et doivent être clairement identifiés. Tout acteur ayant à prendre une décision ou devant contrôler des activités relatives aux marchés publics doit avoir les capacités nécessaires pour mener à bien sa mission. Cette identification permet de s'assurer de la capacité des agents de ces organes de contrôle et de décision dans le domaine des marchés publics et ainsi éviter toute interprétation des textes réglementaires pouvant engendrer des points de blocages. En Bulgarie par exemple, le manque de compréhension des aspects environnementaux des marchés publics par la Cour des comptes a causé plusieurs blocages de projets financés par les fonds européens (OCDE, 2016[1]).

En Algérie, les acteurs publics impliqués dans le système de passation des marchés publics incluent :i) les membres des commissions de marchés compétentes (en fonction de seuils déterminés dans le DMP), qui ont pour rôle d'apporter leur assistance en matière de préparation et de formalisation des marchés publics, d'examiner les projets de cahiers des charges, de marchés et d'avenants, et d'examiner les recours introduits par les soumissionnaires ; ii) les membres de la commission d'ouverture des plis et d'évaluation des offres, issus du service contractant, chargés de l'analyse administrative, technique et financière des offres ; et iii) le contrôleur financier, qui vérifie la disponibilité du budget octroyé au projet de marché public ainsi que la conformité du projet aux dispositions législatives.

Par ailleurs, il convient également d'identifier les agents appartenant à d'autres organes de contrôle et en particulier ceux issus de l'IGF et de la Cour des comptes. L'IGF intervient lors des missions de contrôle d'audit, d'évaluation, d'enquête et d'expertise sur la passation des marchés publics. La Cour des comptes s'assure de l'utilisation efficiente des deniers publics, de l'obligation de rendre des comptes et de la transparence dans la gestion des finances de l'État. Le développement de la stratégie nationale de renforcement des capacités dans le domaine des marchés publics nécessitera de procéder également au recensement des agents impliqués dans le contrôle des marchés publics afin de pouvoir mener des actions ciblées.

L'identification des opérateurs économiques et fournisseurs des entités publiques est également nécessaire afin de pouvoir mener des actions de renforcement des capacités ciblées. En Algérie, il n'existe aucun registre national des fournisseurs de l'État ou des opérateurs économiques pertinents. De tels registres peuvent exister au niveau de certaines entités publiques, mais les informations ne sont pas centralisées (voir chapitre 1). Cependant, il existe de nombreuses organisations patronales ou fédérations 
professionnelles qui peuvent servir de source d'informations. L'Algérie devrait donc identifier clairement les organisations patronales et les fédérations professionnelles ou autre acteurs pertinents afin de comprendre les besoins des opérateurs économiques en termes de renforcement des capacités.

\section{L'identification adéquate des besoins des acteurs impliqués dans le domaine des marchés publics}

Le développement d'une stratégie de renforcement des capacités dans le domaine des marchés publics doit comporter une analyse préalable des besoins. Cette analyse peut être menée en se focalisant sur trois aspects, liés individuellement ou conjointement à la capacité des effectifs en charge des marchés publics : l'analyse des caractéristiques du personnel en charge des marchés publics ; l'analyse de la performance du système de passation des marchés publics et l'analyse du système de formation en place (OCDE, 2017[2]). Lors de cette analyse, il est nécessaire d'identifier les difficultés rencontrées et les erreurs fréquemment commises, d'évaluer les compétences et les connaissances, d'analyser la structure et les méthodes de travail des différents acteurs du système. Ceci permet d'identifier les domaines et sujets qui nécessitent un renforcement des capacités, afin de les intégrer dans les objectifs de la stratégie nationale de renforcement des capacités dans le domaine des marchés publics. (OCDE, 2016[3])

Cette analyse devra dans un premier temps se pencher sur l'évaluation des besoins des institutions, des fonctionnaires (agents en charge de la passation des marchés publics, agents des corps de contrôle et tous autres agents concernés), des opérateurs économiques. La méthode de collecte des données et des informations devra être adaptée au contexte du pays (OCDE, 2016[6]). Les sources d'information peuvent être les rapports des organes de contrôle, de l'entité en charge du système de passation des marchés publics, des organes de recours, des instituts de formations, ou encore des questionnaires.

En Algérie, l'identification des besoins des différentes parties prenantes, et en particulier des agents en charge de la passation des marchés publics, est limitée. Cependant, le pays dispose de nombreuses sources possibles d'informations afin d'obtenir des données fiables à exploiter. Les rapports annuels des organes de contrôle sur les marchés publics que sont l'IGF et la Cour des comptes sont ainsi des sources d'informations pertinentes pour analyser les dysfonctionnements de l'ensemble du cycle de passation des marchés publics. En effet, ces rapports couvrent les aspects réglementaires des marchés publics, mais aussi les aspects pratiques et ceux liés à l'intégrité, de plus ils concernent l'ensemble des acteurs du cycle. Leur analyse servira de base pour mettre en place des actions de renforcement des capacités permettant de corriger ces défaillances pour l'ensemble des parties prenantes.

Dans l'attente de la mise en place de l'ARMP, une autre source importante d'information est la Division des marchés publics du ministère des Finances. En effet, lorsqu'une entité publique rencontre des doutes sur sa compréhension de certaines dispositions du DMP, elle peut saisir la Division des marchés publics pour avis (voir section 1.1.1). Cette dernière fait actuellement office d'autorité en matière législative et réglementaire dans le domaine des marchés publics, et plus particulièrement sur le DMP et les textes subséquents. La collecte d'informations issues des nombreuses consultations et sollicitations qu'elle reçoit peut permettre d'identifier les besoins de renforcement des capacités sur les aspects législatifs, réglementaires et de procédures dans le domaine des marchés publics.

Les commissions des marchés peuvent également être une source pertinente d'informations, car elles sont impliquées dans le processus de passation d'un marché publics, depuis son élaboration jusqu'à sa signature, et peuvent donc identifier les besoins - réglementaires ou pratiques -de renforcement des capacités.

Enfin, la mise en place de questionnaires types, adaptés à l'entité adjudicatrice (soumise au DMP), de la catégorie et du secteur d'activité des opérateurs économiques et de leur localisation géographique (niveau national, régional, etc.) permettront de collecter les informations désirées. 
Afin de mener une stratégie de renforcement des capacités nécessaires au bon fonctionnement du système de passation des marchés publics, l'Algérie gagnerait à mener une analyse préalable des besoins des acteurs, ainsi qu'une évaluation du système de formation. Ces démarches pourront se faire grâce à une collecte d'informations auprès des entités énumérées ci-dessus, la collecte et l'analyse pourront être menées par l'ARMP.

Par ailleurs, une stratégie nationale de renforcement des capacités dans le domaine des marchés publics doit être développée en cohérence avec les différentes stratégies et politiques définies par le gouvernement. En effet, comme décrit au chapitre 5, les marchés publics peuvent être utilisés comme levier stratégique pour atteindre des objectifs de politiques publiques (OPP), comme par exemple des objectifs de développement durable. Pour assurer la prise en compte de ces OPP les gouvernements devraient renforcer les capacités des acteurs du système de passation des marchés publics (OCDE, 2016[9]). Ainsi, par exemple, afin d'assurer l'implémentation des politiques environnementales dans les marchés publics, le Canada a renforcé les capacités des agents chargés de la passation des marchés publics sur les aspects achats durables en mettant notamment en place des guides, des outils et des formations gratuites en ligne (OCDE, 2014[10]). L'Algérie devrait intégrer les objectifs prioritaires et stratégies du gouvernement dans sa stratégie de renforcement des capacités dans le domaine des marchés publics afin d'assurer une cohérence avec les politiques nationales.

\subsection{Mener des actions concrètes pour le renforcement des capacités des acteurs du système de passation des marchés publics}

\subsubsection{Renforcer la professionnalisation des agents chargés des marchés publics}

\section{Reconnaître le métier d'acheteur et mettre en place un cadre de compétence}

Un système de passation des marchés publics repose sur des agents qualifiés pour mener à bien les procédures tout au long du cycle de passation des marchés publics. Cette fonction, de plus en plus considérée comme une activité stratégique et non plus seulement administrative, a pour but de permettre aux États et aux entités publiques une meilleure prestation de services publics aux citoyens. Pour cela, comme souligné dans la Recommandation du Conseil de l'OCDE sur les marchés publics, il est nécessaire de reconnaître le métier d'acheteur public comme un métier spécifique afin de veiller à ce que les acheteurs publics satisfassent aux exigences professionnelles élevées en termes de connaissances, de savoir-faire et d'intégrité (OCDE, 2015[4]).

En effet, l'achat public a longtemps été considéré comme une fonction administrative dont l'objectif principal était le respect de la réglementation des marchés publics. Les marchés publics sont de plus en plus considérés comme un levier de performance économique, mais également comme un levier stratégique pour atteindre des OPP (EPSA Groupe, 2017[11]). Cette évolution a impacté le métier d'acheteur public: tout en nécessitant une expertise et une connaissance de la réglementation des marchés publics, il requiert dorénavant des compétences pluridisciplinaires (économique, marketing, finances publiques, droit, gestion des risques, etc.) (OCDE, 2017 $\left.{ }_{[5]}\right)$. Ce métier est également considéré comme un métier à risques, notamment les risques d'atteinte à l'intégrité, les risques économiques environnementaux et sociaux. Conscients de cette évolution, plus de $60 \%$ des pays membres de l'OCDE ont reconnu cette fonction comme un métier spécifique (voir graphique 6.3). 
Graphique 6.3. La reconnaissance du métier d'acheteur public comme un métier spécifique dans les pays membres de l'OCDE

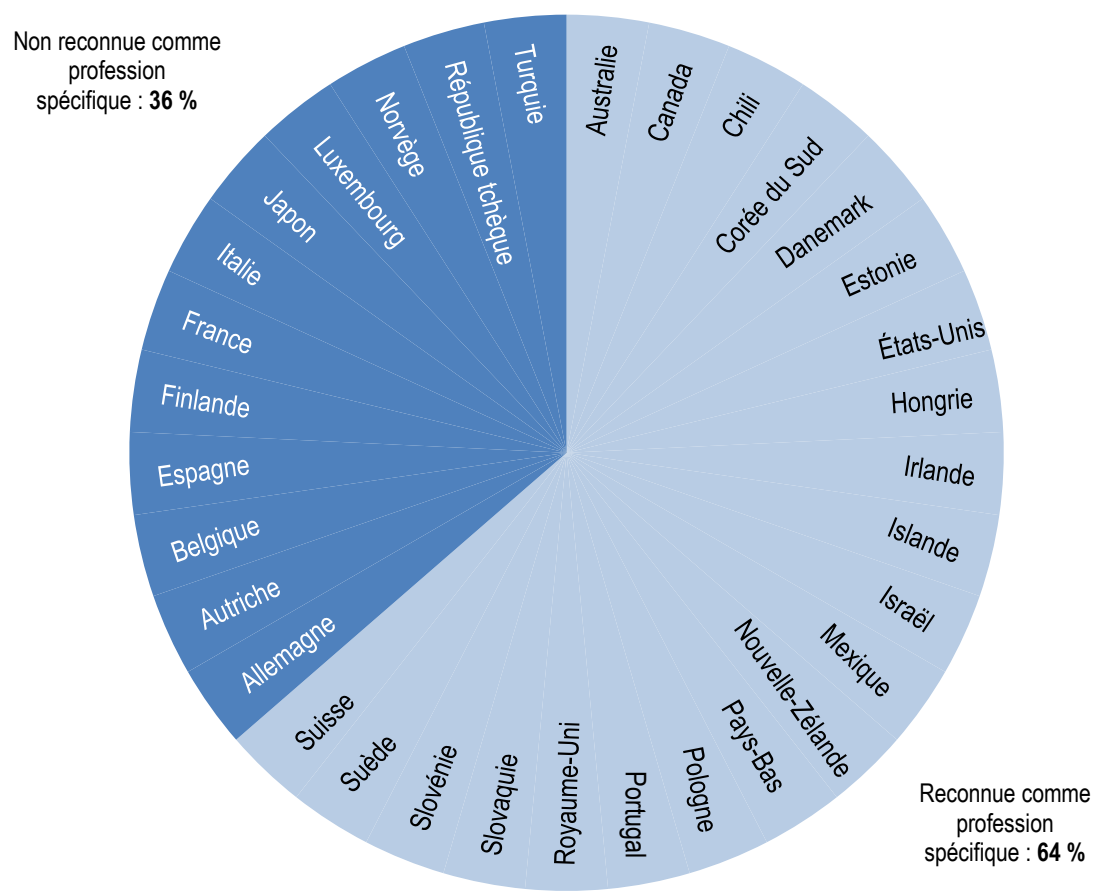

Source : $(O E C D, 2013[12])$.

En Algérie, le métier d'acheteur public n'est pas reconnu comme une profession à part entière. Les métiers de la fonction publique algérienne, sont répartis en quatre groupes (de $A$, le plus élevé, à $D$ ), qui correspondent aux niveaux de formation minimale requis pour y accéder. Les recrutements dans la fonction publique s'effectuent par voie de concours sur épreuves, de concours sur titres pour certains corps de fonctionnaires, de test professionnel, ou encore par recrutement direct parmi les candidats ayant suivi une formation spécialisée prévue par les statuts particuliers, auprès d'établissements de formation habilités.

Tout fonctionnaire ou agent public, quel que soit son groupe (A à $D)$, peut être amené à occuper des fonctions ou à effectuer des tâches liées aux marchés publics. Ces tâches peuvent être occupées à plein temps ou à temps partiel, en plus d'autres tâches annexes ou principales non liées aux marchés publics. D'après les discussions lors des missions exploratoires de l'Organisation de coopération et de développement économiques (OCDE), lorsqu'une entité publique a un besoin en personnel chargé de la passation des marchés publics, elle doit recruter des candidats ayant été reçus au concours de la fonction publique pour un métier en relation avec les finances publiques. En effet, le métier d'acheteur public n'étant pas reconnu comme profession spécifique, il n'existe pas de concours ou examens propres à cette fonction. En ce qui concerne les entreprises publiques économiques (EPE), le personnel chargé des achats n'a pas le statut de fonctionnaire et est recruté hors concours. Ce mode de recrutement permet aux entités concernées d'obtenir plus de flexibilité dans la recherche d'expertise particulière.

L'Algérie gagnerait à reconnaître le métier d'acheteur comme une profession spécifique afin de prendre en considération l'évolution de ce métier d'une fonction administrative à une fonction stratégique pluridisciplinaire, et les risques auxquels il est exposé. En effet, la reconnaissance du métier d'acheteur public permettra également d'organiser des concours ou tests de recrutement spécifiques à ce métier. 
Par ailleurs, l'Algérie pourrait également étudier la possibilité de recourir au recrutement direct des acheteurs ayant suivi une formation spécialisée auprès d'établissements habilités (voir la section 6.2.2).

En plus de la reconnaissance du métier d'acheteur public comme profession spécifique, il est nécessaire de développer un cadre de compétences qui permettra de déterminer les aptitudes et les compétences essentielles pour remplir cette fonction. Ces compétences devront couvrir les aspects professionnels, techniques, mais aussi personnels (travail en équipe, communication, capacités d'encadrement et de management de projets, etc.). L'intégrité doit également être prise en compte comme une compétence essentielle. La maîtrise des outils informatiques peut aussi être requise pour des fonctions achats spécifiques, ainsi que pour utiliser le système électronique de passation de marchés publics. Ces compétences et aptitudes doivent être identifiées dans un but de recherche de performance du système de passation des marchés publics et listées pour chaque métier lié à ce domaine. Un cadre de compétence permet non seulement de déterminer les capacités demandées aux agents publics occupant des fonctions liées aux achats publics, mais peut aussi être utilisé à différentes étapes du processus de gestion des ressources humaines: le recrutement, la promotion, la formation et l'évaluation des performances (OCDE, 2017[5]).

De nombreux pays ont développé de tels cadres de compétences. Le gouvernement écossais a ainsi mis en place un cadre de compétences identifiant les niveaux d'aptitudes et de compétences requis pour tous les agents impliqués dans le processus de passation des marchés publics, et permettant à ces mêmes agents de s'approprier leur développement personnel grâce à des évaluations de compétences, l'identification en besoin de formations et la gestion de leur carrière. Ce cadre de compétences lié à la passation des marchés publics permet également de favoriser les talents qui évoluent déjà dans la fonction publique. II comprend 13 compétences faisant référence à des aptitudes techniques nécessaires à différents niveaux pour mener à bien un processus de passation de marchés publics (voir encadré 6.4). 


\section{Encadré 6.4. Le cadre de compétences des achats publics en Ecosse}

Ce cadre de compétence a été développé suite aux recommandations de la revue du système de passation des marchés publics en Ecosse (2006) et approuvé et utilisé par de nombreuses entités d'achats publics, nationales ou locales. II vient renforcer, et non remplacer, les outils de développement personnel déjà existant au sein des entités. II comprend 13 compétences, et notamment :

- Processus achat : l'agent possède une connaissance et une compréhension suffisantes des méthodes de veille de marché et de soumission pour mener à bien les tâches associées.

- Négociation : l'agent dispose des aptitudes nécessaires à la négociation.

- Développement de stratégie et d'analyse de marché : l'agent dispose des compétences requises pour développer une stratégie et analyser un marché.

- Finances : l'agent dispose des compétences et connaissances nécessaires pour mener à bien les tâches associées dans le cadre de sa fonction, incluant évaluation de la situation financière des fournisseurs, les notions de coûts totaux, et les cadre de conformité existant entre les achats et les finances publiques.

- Réglementaire : l'agent dispose des compétences et connaissances suffisantes concernant le cadre réglementaire relatif à la passation des marchés publics pour mener à bien les tâches associées.

- Orienté résultat : l'agent est conscient de la contribution des objectifs personnels et d'équipe dans la performance du système et démontre son engagement à les atteindre.

- Systèmes : l'agent dispose des connaissances et compétences des systèmes et des processus utilisés dans les achats de biens et de services. Des systèmes spécifiques peuvent demander des compétences particulières.

- Inventaire, logistique et chaîne d'approvisionnement : l'agent dispose des connaissances et compétences en matière de solutions de gestion des approvisionnements pour mener à bien les tâches associées dans le cadre de ses fonctions. Cela peut inclure la gestion d'inventaire, de logistique, de stockage, etc., particulièrement dans le cas d'entité gérant des stocks.

- Conscience organisationnelle : l'agent comprend clairement les rôles et les responsabilités, comment les achats doivent être organisés et la place qu'ils occupent dans l'organisation.

Source : (OCDE, 2016[6]).

Les compétences et aptitudes identifiées à travers ce cadre de compétences peuvent être utilisées pour le développement de profils de postes. En effet, un cadre de compétence est la combinaison entre les objectifs d'une fonction et les compétences attendues de la part de l'agent occupant la fonction pour atteindre les objectifs. Ces profils de postes permettent un meilleur processus de sélection de candidats et de recrutement d'agents, internes ou externes ; ils peuvent également être utilisés pour les processus de promotion (OCDE, 2013 $\left.{ }_{[13]}\right)$.

En Algérie, d'après les missions exploratoires, il n'existe pas de cadre de compétences clairement défini pour les agents chargés de la passation des marchés publics. De plus, les compétences et aptitudes qui leur sont demandées sont limitées aux finances publiques. L'Algérie pourrait donc, dans la continuité de la reconnaissance du métier d'acheteur public, développer un cadre qui permettrait d'identifier les aptitudes et compétence attendues pour les agents occupant cette fonction, développer des plans de formation et de carrière, et adapter les outils de recrutement en corrélation avec ce cadre. 


\section{Favoriser les évolutions pour les agents chargés des marchés publics grâce à des plans de} carrière

La forte rotation des agents en charge de la passation des marchés publics est l'une des problématiques des entités publiques. En effet, en l'absence de plan de carrière, ces agents évoluent vers d'autres métiers de la fonction publique ou le secteur privé. Afin de limiter les départs, des mesures doivent être mises en place pour prendre en compte la complexité du métier d'acheteur public et les risques qu'il comporte, sécuriser les agents et assurer une pérennité des métiers liés à la passation des marchés publics. Parmi ces mesures figurent notamment le développement de plans de carrière et une valorisation salariale. Ces éléments étant liés au cadre de la fonction publique, l'institution chargée de la fonction publique concernée doit être impliquée dans la stratégie de renforcement des capacités (voir paragraphe 6.1.1.) (OCDE, 2016[6]).

De nombreux pays ont mis en place des plans de carrière pour les agents en charge des marchés publics en créant différents métiers liés à la fonction " achat public » de façon à permettre aux acheteurs publics d'envisager une progression professionnelle. L'encadré 6.5 décrit les évolutions de carrière du métier d'acheteur public en France.

\section{Encadré 6.5. Les métiers liés à l'achat public en France}

En France, le Répertoire interministériel des métiers de l'État (RIME), regroupe l'ensemble des emplois de l'État. II permet l'identification des emplois, des activités et des compétences, et est utile pour l'organisation des services et la gestion des parcours professionnels des agents.

Les métiers de l'État sont répartis en 28 domaines fonctionnels, comptant au total 282 métiers. Le domaine fonctionnel « achat » compte les cinq métiers suivants:

- Acheteuse/acheteur : Procéder à des achats de toute nature en vue de satisfaire les besoins qualitatifs et quantitatifs des services et contribuer à la performance des achats.

- Approvisionneuse/approvisionneur: Assurer et gérer l'approvisionnement en biens et services dans le cadre de l'exécution des marchés et des politiques d'achats.

- Conseillère/conseiller en ingénierie d'achat : Conseiller et apporter son expertise aux acteurs de la chaîne achat en vue d'optimiser l'achat public et de contribuer à sa performance.

- Rédactrice/rédacteur de la commande publique : Mettre en œuvre les procédures de marchés publics pour répondre à un projet d'achat et conseiller les acheteurs sur les risques et/ou bénéfices des procédures engagées.

- Responsable achat : Piloter, mettre en œuvre la politique achat au sein de son entité selon les objectifs de performance assignés par l'État et définir les stratégies d'achat relevant de son périmètre.

Source : (Gouvernement de France, 2017 $[14])$.

En Algérie, l'article 111 du Statut général de la fonction publique prévoit que la gestion de carrière des fonctionnaires est assurée dans le cadre d'une politique de gestion prévisionnelle des ressources humaines consacrée à travers des plans annuels de gestion des ressources humaines et des plans annuels ou pluriannuels de formation et de perfectionnement (Gouvernement d'Algérie, 2006[15]). Cependant, le métier d'acheteur n'étant pas reconnu comme une profession spécifique, la gestion de carrière des agents ayant des fonctions liées aux marchés publics est limitée. Les missions exploratoires montrent que les agents chargés des marchés publics peuvent être amenés, dans le cadre de leur 
évolution de carrière, à occuper des fonctions dans un domaine différent. En plus de la reconnaissance du métier d'acheteur public comme profession spécifique, l'Algérie pourrait également considérer la possibilité de développer un plan de carrière pour les agents publics qui occupent cette fonction en établissant des catégories de postes d'acheteurs publics de façon à permettre aux agents en charge de la passation des marchés publics de valoriser et de faire évoluer leurs compétences et leur carrière au sein de la fonction publique.

\section{Mettre en place une rémunération en adéquation avec la complexité du métier d'acheteur public}

Le métier d'acheteur n'étant pas reconnu comme une profession spécifique en Algérie, la rémunération des fonctionnaires en charge des marchés publics ne prend pas en considération les spécificités du métier et est indexée selon les grilles de rémunération de la fonction publique. Pourtant, la rémunération des acheteurs publics devrait refléter la complexité de leur métier (procédures, compréhension et application du cadre législatif), les risques auxquels ils font face et les compétences multiples nécessaires (notions de droit, d'économie, de comptabilité, de marketing, etc.). Toute réforme concernant la passation des marchés devra donc être accompagnée d'une refonte des conditions de travail et de la rémunération des acheteurs publics afin d'attirer à la profession des agents qualifiés, de les motiver pour une meilleure performance de l'ensemble du système de passation des marchés publics, et de réduire les probabilités de corruption (UNPCDC, 2011[16]).

Actuellement, la rémunération est composée de deux éléments : le traitement, basé sur la grille indiciaire générale de la fonction publique, bâtie selon le niveau de diplôme et les primes (qui rémunèrent le rendement et la performance) et les indemnités (qui rémunèrent des sujétions particulières inhérentes à l'exercice de certaines activités ainsi qu'au lieu et aux conditions spécifiques de travail) (Gouvernement d'Algérie, 2007[17]). D'après les missions exploratoires, un agent en charge des marchés publics en début de carrière gagne environ 250 EUR (euros, soit environ 34000 DZD [dinars algériens]) par mois. Cette rémunération peut varier d'un ministère à un autre ou d'une entité publique à une autre en fonction des primes et indemnités. Ces agents peuvent être amenés à gérer des marchés à haut risque, comme le souligne la loi $n^{\circ} 06-01$ du 20 février 2006 relative à la prévention et la lutte contre la corruption, qui recommande une rémunération en adéquation avec les risques auxquels un fonctionnaire est exposé.

Le gouvernement algérien devrait donc réfléchir à mettre un système de rémunération incitatif clair, transparent et valorisant les fonctions liées à la passation des marchés publics en prenant en compte la complexité des procédures et les différents risques auxquels sont exposés les agents publics qui occupent ces fonctions.

\subsubsection{Des formations adaptées aux besoins des acteurs du système de passation des marchés publics}

Les formations disponibles pour le renforcement des capacités dans le domaine des marchés publics devraient être développées en prenant en compte les besoins spécifiques des agents publics en charge de la passation des marchés publics et de l'ensemble des parties prenantes. Ces formations devraient être en adéquation avec les métiers impliqués dans le processus de passation des marchés publics et être développées de manière à renforcer les compétences et les aptitudes nécessaires pour une bonne gestion des marchés publics. En plus des aspects réglementaires encadrant le cycle de passation des marchés publics, les formations devraient également couvrir l'aspect pluridisciplinaire des achats publics (OCDE, 2017[5]).

En Algérie, le statut général de la fonction publique prévoit dans les articles 104 et 105, la formation permanente des fonctionnaires, dont l'organisation est confiée à l'administration (Gouvernement d'Algérie, $\left.2006_{[15]}\right)$. De plus, l'article 3 de la loi $n^{\circ}$ 06-01 du 20 février 2006 relative à la prévention et à la lutte contre 
la corruption prévoit également que des formations soient dispensées aux fonctionnaires pour la sensibilisation aux risques de corruption. Enfin, les articles 211 et 212 du DMP précisent que les agents en charge de la passation des marchés publics doivent recevoir des formations qualifiantes dans le domaine des marchés publics et bénéficier de cycles de formation, de perfectionnement et de recyclage assurés par l'organisme employeur en relation avec l'ARMP. La création effective de l'ARMP permettrait à l'Algérie de renforcer son système de passation des marchés publics.

L'évaluation du système de formation permet de mesurer la variété et le degré de spécialisation des formations existantes dans le domaine des marchés publics et d'analyser leurs résultats. L'évaluation peut comprendre l'étude de plusieurs indicateurs comme, par exemple, les compétences et l'expérience des formateurs, la durée des formations, la population cible des formations, le type de formation (sur site ou en ligne), les aspects des achats enseignés, la reconnaissance des formations (OCDE, 2016[6]). L'encadré 6.6 décrit l'analyse du système de formation dans le domaine des marchés publics en République slovaque.

\section{Encadré 6.6. Analyse du système de formation en marchés publics en République slovaque}

L'OCDE a analysé le système de formation en marchés publics en République slovaque. Basée sur des questionnaires, cette analyse se décline en deux parties : une première partie concerne le cadre institutionnel de formation, une seconde partie concerne le portefeuille de formations disponibles.

\section{Le cadre institutionnel de formation}

Comprendre le cadre institutionnel de formations est nécessaire afin de développer un plan d'action de formation efficace, identifiant ainsi des synergies potentielles dans le développement de matériel de formation et/ou dans l'identification de formateurs expérimentés.

À travers une série de questions les institutions qui fournissent des formations, les politiques et les exigences en matière de formations, et les coûts associés, un bilan de la situation a pu être dressé ainsi que l'impact de l'implémentation d'un plan de formation sur le système actuel.

Si une évaluation du nombre de participants aux formations ainsi que du nombre de formations permet au Bureau des marchés publics de la République slovaque d'évaluer son activité de formation, il est nécessaire de prendre en considération la typologie et le nombre d'agents en charge marchés publics afin de disposer d'une orientation suffisante sur la progression des activités de formations.

\section{Le portefeuille de formations}

Une analyse de la formation se concentre également sur la structure et le contenu du portefeuille de formations disponibles afin d'évaluer son adéquation avec le système d'achats publics et ses objectifs. Un aperçu des sujets couverts par les formations permet d'avoir une indication sur les compétences développées par les agents en charge de la passation des marchés publics.

Comme pour l'évaluation du cadre institutionnel, une série de questions a été posée sur les sujets suivants: la visibilité de la formation, sa structure, le portefeuille de formations, le format des formations, leur fréquence, et enfin leur efficacité.

Source : adapté de (OCDE, 2017[2]). 


\section{Développer l'offre de formations en présentiel et s'assurer de leur qualité}

En Algérie, plusieurs centres de savoir dispensent des formations sur les marchés publics. Le principal organisme dans ce domaine est l'ISGP, une entité sous tutelle du ministère des Finances qui offre des formations à destination du secteur public et du secteur privé. Basée à Alger et disposant d'un effectif d'une dizaine de formateurs ainsi que d'une vingtaine de consultants, I'ISGP délivre quatre types de programmes sur les marchés publics, de différents niveaux, accessibles pour l'ensemble des acteurs du système des marchés publics : un diplôme d'études supérieures spécialisées (DESS) en management des marchés publics, un certificat de maîtrise de la réglementation des marchés publics, des séminaires spécialisés (formations en cycles courts en compléments aux formations précédemment citées). Enfin elle propose également une formation courte ayant pour thème « Les zones de prédilection de la fraude dans les marchés publics » dans le cadre du programme national de formation et de sensibilisation anticorruption.

L'ensemble de ces formations sont surtout axées sur les aspects réglementaires des marchés publics et n'ont concerné qu'un total de 720 personnes pour le DESS et 3000 personnes pour les formations courtes. Ces formations ont pu être dispensées lors de la prise de fonction des agents concernés ou durant leur carrière, et ce quel que soit leur niveau de responsabilité. Ce chiffre est relativement faible compte tenu de l'ensemble des pouvoirs adjudicateurs potentiels en Algérie répartis parmi 28 ministères et 48 wilayas (collectivités publiques territoriales), elles-mêmes divisées en 548 daïra comprenant en tout 1541 communes. Par ailleurs, ces formations ne sont disponibles qu'à Alger et, d'après les missions exploratoires, les déplacements en dehors d'Alger sont très limités. L'ISGP devrait donc développer le nombre de formations qu'elle propose, mais aussi les sujets traités par ces formations de façon à refléter l'évolution du domaine des marchés publics d'une activité administrative vers une activité stratégique. Ces formations devraient donc refléter l'aspect pluridisciplinaire des marchés publics en couvrant les aspects réglementaires mais également les aspects pratiques et stratégiques.

II existe également d'autres actions de renforcement de capacités sur les marchés publics par des consultants du secteur privé ou au sein même de certains établissements publics, comme par exemple le groupe Cosider, entreprise publique du secteur du bâtiment, travaux publics et hydrauliques, qui a créé sa propre école de formation en interne, dont les thèmes abordent les marchés publics. Cependant, d'après les missions exploratoires, la qualité de certaines de ces formations n'est pas suffisante. Des pays comme le Pérou ont mis en place un système de certification des organismes de formation, et leur fournit les modules de formations. (OCDE, 2017[5])

L'Algérie devrait envisager la possibilité de certifier des organismes dispensant des formations dans le domaine des marchés publics afin d'assurer un niveau de qualité adéquat et l'atteinte des objectifs de la commande publique. L'ARMP et des délégations de service public pourraient délivrer ces certifications.

Par ailleurs, des questionnaires sont remis aux élèves à l'issue des formations dispensées par l'ISGP. Cependant, d'après les missions exploratoires, l'utilisation de ces questionnaires pour mener une évaluation du système de formation semble limitée. Les informations quant à l'évaluation des autres formations dispensées par d'autres organismes de formation publics ou privé ne sont pas disponibles. L'Algérie devrait donc mettre en place des mécanismes permettant l'évaluation systématique des formations afin d'assurer leur qualité et leur adéquation avec les besoins de formations des bénéficiaires.

\section{Permettre au secteur privé de renforcer ses capacités dans le domaine des marchés publics}

L'amélioration du système de passation des marchés publics nécessite également le renforcement des capacités du secteur privé. En effet, en tant qu'utilisateurs du système, ils se doivent de mieux comprendre le cadre réglementaire, mais aussi les aspects pratiques de ce domaine. Le renforcement de leurs 
capacités permet d'améliorer et d'augmenter leur participation aux marchés publics, mais également de limiter certains risques en cours d'exécution du marché (EBRD, 2012[18]).

En Algérie, le secteur privé ne dispose que de très peu d'offres de formation ayant pour thème les marchés publics. En effet, les formations de l'ISGP ciblent principalement le secteur public et ne répondent donc pas directement à leur besoin en tant que soumissionnaires ou fournisseurs d'un contrat avec des entités publiques. Par ailleurs, la Chambre algérienne de commerce et d'industrie ( $\mathrm{CACl}$ ) a organisé des séminaires pour apporter des clarifications sur le DMP (deux sessions de deux jours en novembre et décembre 2015), mais ces actions restent ponctuelles et focalisées sur les aspects réglementaires, elles n'abordent pas les aspects pratiques des marchés publics et ont, de plus, lieu principalement à Alger. Les missions exploratoires montrent par ailleurs que les actions menées par les organisations professionnelles et patronales restent limitées. Certains pays ont développé des formations agréées par l'État pour le secteur privé en collaboration avec des acteurs du secteur privé tels que les associations professionnelles. C'est le cas par exemple de l'Italie, qui a mis en place des formations à destination du secteur privé en collaboration avec des associations professionnelles pour l'utilisation de son système électronique de passation de marché public, et ce, à travers tout le pays (Consip, Italie, 2016[19]). Afin de renforcer les capacités du secteur privé dans le domaine des marchés publics, l'Algérie devrait développer des formations en collaboration avec des associations professionnelles ou fédérations patronales, comme par exemple le Forum des chefs d'entreprises ou l'Association générale des entrepreneurs algériens, de façon à répondre spécifiquement à leurs besoins. Ce type de collaboration sera également nécessaire pour la mise en place effective du portail électronique de passation des marchés publics.

\section{Utiliser les outils d'enseignement à distance}

L'utilisation des moyens d'information et de communication pour renforcer et/ou assister l'apprentissage est une pratique à encourager car elle permet d'améliorer l'accessibilité aux opportunités de formation en s'affranchissant des contraintes de temps et de lieu de formation. II est en effet tout aussi important de développer des formations de qualité que de permettre l'accès à ces formations. Cet accès peut être favorisé en diffusant de manière adéquate les opportunités de formation et en s'assurant que les offres de formations sont disponibles pour les agents en charge des marchés publics du niveau central mais également du niveau régional et local. Ce type de formations peut également être bénéfique pour le secteur privé. Ces formations devraient être proposées gratuitement ou à moindre coût et utiliser les nouvelles technologies de l'information et de la communication. L'accès aux informations sur les formations devront être disponibles aussi bien pour les agents publics que pour le secteur privé, (OCDE, 2017[5]). Les coûts associés au développement d'outils d'enseignement à distance peuvent varier en fonction de la complexité des modules développés et des technologies utilisées. Les investissements initiaux liés au développement et à la mise en place de tels outils peuvent rapidement permettre des économies en fonction du nombre de participants (OCDE, 2017[2]).

Plusieurs pays développent l'enseignement à distance, notamment le e-learning, pour les agents en charge de la passation des marchés publics (voir l'exemple de la Norvège, encadré 6.7). 


\section{Encadré 6.7. La formation en ligne sur les marchés publics en Norvège}

Reconnaissant la nécessité de renforcer les capacités et la formation des agents du secteur public, et face aux coûts inhérents aux méthodes traditionnelles, l'Agence pour la gestion publique et l'administration en ligne (Difi) de Norvège a développé une plateforme de formation en ligne.

Lancée en juin 2016, la plateforme https://laeringsplattformen.difi.no/ propose des cours en ligne aux entités publiques et à leurs employés, et leur permet de partager leurs supports de formation avec d'autres entités publiques. Les cours sont accessibles à tous, gratuitement. Des enquêtes ont montré qu'une vaste majorité des participants $(80 \%)$ apprécient ce moyen de formation, qui peut être déployé sans considération de temps ou de lieu. La gestion d'une majeure partie de la formation en ligne par une unité centrale a permis une meilleure gestion des compétences.

Des nombreux avantages financiers ont été obtenus notamment par une meilleure utilisation des ressources et par une diminution du nombre de prestations de formations.

La plateforme norvégienne propose quatre formations différentes en achat public :

- La bonne utilisation des achats publics

- Comment éviter le dumping social dans les achats publics

- Coût du cycle de vie pour la construction et le bâtiment

- Guide sur les contrats publics standards

Source : adapté de (OCDE, 2017[5]).

D'autres outils d'enseignement à distance sont disponibles, comme par exemple les formations en ligne ouvertes à tous (Massive Online Open Courses [MOOC]), qui peuvent accueillir un grand nombre de participants dispersés géographiquement et communiquant uniquement par Internet (UNESCO, 2018[20]). De nombreux MOOC sur le thème des marchés publics sont disponibles, comme par exemple les programmes de certification en achat public développés par la Banque mondiale (World Bank, 2014[21]). Ces outils de e-learning ne sont pas réservés aux agents en charge de la passation des marchés publics, ils sont accessibles à tous y compris le secteur privé.

En Algérie, malgré l'entendue géographique du pays, ce type d'enseignement à distance n'est pour le moment pas disponible. Cependant, l'ISGP développe actuellement un module d'enseignement à distance avec l'Organe national de prévention et de lutte contre la corruption (ONPLC) sur le thème de la prévention et de la lutte contre la corruption, cette formation sera disponible en français et en arabe. C'est une bonne initiative qui permettra de former un maximum d'agents publics et couvrir l'ensemble du territoire.

L'Algérie pourrait tirer bénéfice de cette bonne initiative en poursuivant le développement de formations en ligne dans le domaine des marchés publics, que ce soit grâce au e-learning ou aux MOOC, qui devront couvrir l'ensemble des aspects des marchés publics et qui permettront de mettre ces formations à disposition du plus grand nombre possible d'agents publics, et ce, sur la totalité du territoire. L'accès à ces formations dématérialisées devra également être disponible pour les acteurs du secteur privé.

\section{Mettre en place des formations de formateurs}

Une stratégie nationale de renforcement de capacités dans le domaine des marchés publics doit proposer des formations de qualité à l'ensemble des parties prenantes. À cette fin, il est nécessaire de développer des formations de formateurs, qui devront être choisis parmi des acheteurs ou autres agents publics experts en marchés publics. Pour être pertinents, ces programmes devront être centrés sur le contexte 
national du système de passation des marchés publics, mais les formateurs pourront également suivre des formations ayant une portée internationale qui permettront les comparaisons afin de développer leurs supports de formation et de perfectionner leur pédagogie (OCDE, 2016[6] $)$.

Plusieurs pays ont mis en œuvre des plans de formation de formateurs, comme par exemple le Maroc qui, avec l'appui de l'OCDE, a organisé des formations de formateurs sur le thème de l'intégrité dans les marchés publics (ICPC, s.d.[22]). La mise en place de telles initiatives nécessite une approche méthodologique précise, décrite dans l'encadré 6.8 en s'appuyant sur l'expérience de la Bulgarie.

\section{Encadré 6.8. Formation de formateurs en Bulgarie}

Dans le cadre du projet Support pour la conception et l'implémentation du programme Développement et formation achats publics en Bulgarie financé par l'Union européenne (UE), la Bulgarie a développé, en collaboration avec l'OCDE, un programme de formation de formateurs incluant des ateliers de formation et des sessions pilotes.

\section{Formation de formateurs}

L'objectif principal des ateliers est de former des experts locaux en achats publics aux techniques de formation et à l'utilisation de moyens modernes de formation. II s'agit de doter les agents en charge de la passation des marchés publics de solides bases sur la réglementation des marchés publics en Bulgarie adoptée en 2016, mais également sur les aspects méthodologiques de formation.

\section{Mise en place de l'atelier de formation}

La mise en place de l'atelier de formation pour les formateurs a été développée selon les conseils et les lignes directrices fournis par les parties prenantes locales et les responsables du projet. Des pairs et experts de pays européens ont été invités à partager leurs expériences sur des cas concrets.

\section{Évaluation des participants, des ateliers et des formateurs}

Durant la formation, les participants ont été régulièrement testés sur les différents sujets enseignés à la fin de chaque jour de formation.

\section{Matériel de formation pour le programme Formation de formateurs}

Le matériel développé dans le cadre de ce projet ne l'a pas été uniquement pour les ateliers de formation, il peut également servir aux agents en charge de la passation de marchés publics.

\section{Mise en place des sessions pilotes}

L'objectif des sessions pilotes était de permettre à chaque participant (les formateurs) de présenter l'intégralité ou une partie d'un module. II s'est tenu deux sessions pilotes : la première a permis d'affiner les performances des formateurs ainsi que le contenu de leur support de formation. La seconde a été l'occasion de mesurer la progression des prestations des formateurs et d'évaluer si les supports de formations nécessitaient des améliorations.

Source : adapté de (OCDE, 2016[1]).

Actuellement en Algérie, I'ISGP dispense des formations de formateurs sur des sujets liés aux finances publiques, mais pas sur les marchés publics. Étant donné l'étendue géographique du pays et le nombre d'entités adjudicatrices, l'Algérie devrait investir dans un programme de formation de formateurs dans le domaine des marchés publics afin d'atteindre un plus grand nombre de bénéficiaires. 


\subsubsection{Renforcer la collaboration avec les centres de savoir et les universités}

La collaboration avec les centres de savoir, notamment les universités et plus généralement l'enseignement supérieur doit également être développée afin d'améliorer les compétences des acheteurs publics. L'expertise et l'expérience pédagogique des centres de savoir constituent des outils précieux sur lesquels il faudrait s'appuyer pour enrichir les connaissances relatives aux marchés publics, nourrir le dialogue entre la théorie et la pratique (OCDE, 2015[4] $)$. Ces centres de savoir permettent en effet de contribuer au renforcement des capacités des agents en charge des marchés publics ou d'y préparer des étudiants à occuper efficacement des fonctions liées aux marchés publics. De nombreux pays ont développé des diplômes supérieurs ayant pour thème les marchés publics, comme par exemple le Royaume-Uni, où l'université de Nottingham propose un diplôme universitaire en gouvernance et droit des marchés publics (voir encadré 6.9).

\section{Encadré 6.9. Diplôme universitaire en gouvernance et droit des marchés publics de l'université} de Nottingham

Au Royaume-Uni, l'université de Nottingham propose un diplôme universitaire de troisième cycle spécialisé sur les marchés publics. Ce diplôme dispense des modules de tronc commun portant sur :

- les principes de base et les concepts des achats publics

- la réglementation européenne

- la corruption et la collusion dans les marchés publics

- le cadre réglementaire des marchés publics selon l'Organisation mondiale du commerce

- les méthodes permettant de faire de la recherche dans le domaine de la réglementation des marchés publics.

Des modules optionnels permettent d'aborder, au choix, l'organisation et le management de système de passation des marchés publics, des sujets spéciaux liés la réglementation européenne, le lien entre développement économique et marché public, ou encore la réglementation du Royaume-Uni sur les marchés publics.

Source : (Université de Nottingham, Royaume-Uni, s.d.[23]).

En Algérie, il n'existe pas de formations dans l'enseignement supérieur ayant pour thème principal les marchés publics. Ce sujet est généralement traité dans les modules relatifs au droit public. Seule l'École nationale d'administration propose quelques modules dans le domaine des marchés publics, mais pas de formation complète sur ce sujet. L'introduction de formations dans l'enseignement supérieur en Algérie nécessite une décision par décret présidentiel. Afin de renforcer son système de passation des marchés publics, l'Algérie gagnerait donc à introduire les marchés publics dans l'enseignement supérieur en offrant des diplômes spécifiques sur ce domaine.

\subsubsection{Mettre à disposition des acteurs du système de passation des marchés publics des outils de renforcement des capacités}

Afin de s'assurer que les acteurs d'un système de passation des marchés publics satisfassent aux exigences professionnelles élevées en termes de connaissances, de savoir-faire et d'intégrité, l'OCDE recommande qu'un ensemble d'outils régulièrement actualisés soit mis à leur disposition (OCDE, 2015[4]). Ces outils doivent permettre, par exemple, de fournir des lignes directrices sur le cadre politique ou réglementaire de la passation des marchés publics, ou encore de proposer des recommandations en 
termes de bonnes pratiques. Ces outils devraient être proposés par l'entité en charge des marchés publics (fonction centrale) (OCDE, 2016 $\left.6_{[3]}\right)$.

De nombreux pays ou institutions ont mis en place de tels outils au travers de guides ou de lignes directrices, Ils facilitent l'utilisation et la compréhension du cadre réglementaire des marchés publics et limitent les risques d'erreurs en mettant en avant les bonnes pratiques à observer durant le processus de passation d'un marché public. S'ils sont développés en premier lieu pour les agents en charge de la passation des marchés publics, leur publication en ligne permet également de renforcer les capacités de l'ensemble des parties prenantes, fonctionnaires ou opérateurs économiques.

La Commission européenne a ainsi créé un guide des marchés publics à destination des acheteurs publics (Commission Européenne, 2018[24]). Ce guide, gratuit et en libre accès, offre une assistance pratique afin d'éviter les erreurs communément constatées. II aborde les principales étapes d'un cycle de passation des marchés publics : la préparation et la planification ; la publication et la transparence ; la soumission des offres et la sélection des soumissionnaires ; l'évaluation des offres et l'attribution; et la mise en place du contrat. De nombreux pays mettent également à disposition de tels guides ou lignes directrices, c'est le cas par exemple de la France (Direction des affaires juridiques, France, 2018[25]).

En Algérie, certains établissements publics ont mis en place des guides internes de procédures de passation des marchés publics à destination de leurs agents en charge des marchés publics, comme par exemple le groupe Cosider (voir encadré 6.10) :

\section{Encadré 6.10. Manuel de procédures de passation de commande du groupe Cosider (édition juin 2015)}

Le groupe Cosider est une EPE du secteur des bâtiments, des grands ouvrages et des infrastructures de transports. Cette entreprise publique n'est pas soumise aux dispositions du DMP. Elle a cependant mis en place une procédure pour la passation de ses marchés, adaptée de la réglementation des marchés publics : « les adaptations apportées sont en rapport étroit avec la spécificité des activités des sociétés du Groupe et de leur environnement. Le Groupe Cosider a développé ce manuel dans une forme pratique pour servir de guide à tous les acteurs qui interviennent à différents niveaux, dans le processus achat $»$.

Ce guide a été validé en interne par le conseil d'administration et par l'assemblée générale et est disponible en version bilingue arabe/français.

II contient les chapitres suivants :

1. Disposition de la procédure

2. Processus de mise en œuvre

3. Contrôle des marchés

4. Négociation, attribution et recours

5. Dispositions contractuelles

6. Gestion de contrat

7. Lutte contre la corruption

8. Échange d'information par voie électronique

9. Dispositions finales.

Source : (Groupe COSIDER, 2015[26]). 
Lors des missions exploratoires, l'Algérie a mentionné l'existence de tels guides dans d'autres entités publiques ou ministères, soumis ou non au DMP, dont le contenu est validé par les organes de contrôle des entités concernées. Cependant, leur contenu doit être également revu par une entité faisant autorité en matière de marchés publics afin de s'assurer du bon respect du cadre réglementaire en vigueur et de ses principes. En effet, les missions exploratoires ont identifié des problématiques de compréhension du cadre réglementaire, qui sont parfois retranscrites dans les guides des entités. Afin d'assurer une compréhension commune du cadre réglementaire des marchés publics, l'Algérie devrait développer un guide général sur les aspects pratiques du DMP, sous la responsabilité de l'ARMP et de délégations de service public. Le gouvernement algérien développera prochainement, avec le support de l'OCDE, un manuel sur les aspects pratiques du DMP, qui s'adressera aux praticiens des marchés publics - acheteurs publics et fournisseurs - afin de faciliter la lecture, la compréhension et l'interprétation du DMP. Pour établir ce manuel, il est nécessaire de disposer d'un cadre réglementaire stable et pleinement appliqué (voir chapitre 1).

De plus, l'Algérie devrait inciter les entités publiques non soumises au DMP à développer de tels guides qui, en plus d'être adoptés par leurs organes habilités, pourront être revus et validés par l'ARMP.

Par ailleurs, l'article 13 du DMP prévoit un manuel de procédures internes pour la passation des commandes en dessous des seuils (égal ou inférieur à 12 millions DZD pour les travaux ou les fournitures, et 6 millions DZD pour les études ou services). Ces manuels peuvent être différents d'une entité à l'autre. L'ARMP gagnerait à fournir un exemplaire du manuel de procédures internes afin d'assurer une homogénéité dans les pratiques en dessous des seuils (en procédures adaptées). 


\section{Propositions d'action}

\begin{tabular}{|c|c|c|c|}
\hline Recommandations & $\begin{array}{l}\text { Court } \\
\text { terme }\end{array}$ & $\begin{array}{c}\text { Moyen } \\
\text { terme }\end{array}$ & $\begin{array}{l}\text { Long } \\
\text { terme }\end{array}$ \\
\hline Mettre en place l'ARMP et lui confier le pilotage de la stratégie nationale de renforcement des capacités. & $\mathrm{X}$ & & \\
\hline $\begin{array}{l}\text { S'assurer de l'application et la mise en œuvre de l'ensemble des dispositions du DMP et autres textes } \\
\text { réglementaires }\end{array}$ & $\mathrm{X}$ & & \\
\hline $\begin{array}{l}\text { Mettre en place un comité de pilotage afin de développer une stratégie nationale de renforcement des } \\
\text { capacités dans le domaine des marchés publics }\end{array}$ & & $\mathrm{X}$ & \\
\hline Développer une stratégie nationale de renforcement des capacités & & $X$ & \\
\hline $\begin{array}{l}\text { Procéder à l'identification et au recensement de l'ensemble des agents publics en charge de la passation } \\
\text { des marchés publics }\end{array}$ & & $\mathrm{X}$ & \\
\hline Procéder au recensement des agents impliqués dans le contrôle des marchés publics & & $X$ & \\
\hline $\begin{array}{l}\text { Identifier clairement les organisations patronales et les fédérations professionnelles ou autre acteurs } \\
\text { pertinents afin de comprendre les besoins des opérateurs économiques en termes de renforcement des } \\
\text { capacités }\end{array}$ & & $\mathrm{X}$ & \\
\hline $\begin{array}{l}\text { Mener une analyse et une identification préalable des besoins des acteurs du système de passation des } \\
\text { marchés publics et également à une évaluation du système de formation }\end{array}$ & & $\mathrm{X}$ & \\
\hline $\begin{array}{l}\text { Intégrer dans la stratégie de renforcement des capacités les objectifs prioritaires et stratégies du } \\
\text { gouvernement afin d'assurer une cohérence avec les politiques nationales }\end{array}$ & & & $\mathrm{X}$ \\
\hline Reconnaître le métier d'acheteur comme une profession spécifique & & & $\mathrm{X}$ \\
\hline $\begin{array}{l}\text { Recourir au « recrutement direct » des acheteurs ayant suivi une formation spécialisée auprès } \\
\text { d'établissements habilités }\end{array}$ & & & $\mathrm{X}$ \\
\hline $\begin{array}{l}\text { Développer un cadre de compétences afin d'identifier les aptitudes et compétence attendues en matière } \\
\text { de marchés publics }\end{array}$ & & & $\mathrm{X}$ \\
\hline $\begin{array}{l}\text { Développer des plans de formations et de carrière pour les agents en charge de la passation des marchés } \\
\text { publics }\end{array}$ & & & $\mathrm{X}$ \\
\hline Adapter les outils de recrutement en corrélation avec le cadre de compétences & & & $\mathrm{X}$ \\
\hline Établir des catégories de postes d'acheteur public & & & $\mathrm{X}$ \\
\hline $\begin{array}{l}\text { Mettre un système de rémunération incitatif clair et transparent et valorisant les fonctions liées à la } \\
\text { passation des marchés publics }\end{array}$ & & & $\mathrm{X}$ \\
\hline $\begin{array}{l}\text { Développer l'offre de formations de l'ISGP en augmentant le nombre des cours mais également les sujets } \\
\text { traités }\end{array}$ & & $X$ & \\
\hline Certifier des organismes dispensant des formations dans le domaine des marchés publics & & & $X$ \\
\hline Mettre en place des mécanismes permettant l'évaluation systématique des formations & $X$ & & \\
\hline $\begin{array}{l}\text { Développer des formations sur les marchés publics à destination du secteur privé en collaboration avec } \\
\text { des associations professionnelles ou fédérations patronales }\end{array}$ & & $\mathrm{X}$ & \\
\hline $\begin{array}{l}\text { Développer des formations en ligne dans le domaine des marchés publics, également disponibles pour le } \\
\text { secteur privé }\end{array}$ & & & $\mathrm{X}$ \\
\hline $\begin{array}{l}\text { Investir dans un programme de formation de formateurs dans le domaine des marchés publics afin } \\
\text { d'atteindre un plus grand nombre de bénéficiaires. }\end{array}$ & $\mathrm{X}$ & & \\
\hline $\begin{array}{l}\text { Introduire les marchés publics dans l'enseignement supérieur en offrant des diplômes spécifiques sur ce } \\
\text { domaine }\end{array}$ & & & $\mathrm{X}$ \\
\hline $\begin{array}{l}\text { Développer un guide général sur les aspects pratiques du décret 15-247 (sous l'autorité de l'ARMP ou de } \\
\text { la Division des marchés publics du ministère des Finances) }\end{array}$ & $x$ & & \\
\hline $\begin{array}{l}\text { Inciter les entités publiques non soumises au DMP à développer des guides pratiques sur leur procédures } \\
\text { de passation de marchés }\end{array}$ & & $x$ & \\
\hline $\begin{array}{l}\text { Fournir un exemplaire de manuel de procédures internes pour les procédures en dessous des seuils } \\
\text { (procédures adaptées) }\end{array}$ & $\mathrm{x}$ & & \\
\hline
\end{tabular}

Note : Court terme : recommandation à mettre en application dans les 12 mois suivants ;

Moyen terme : recommandation à mettre en application dans les 1-2 ans ;

Long terme : recommandation à mettre en application dans plus de 2 ans. 


\section{Références}

Commission Européenne (2018), Public Procurement Guidance for Practitioners, DirectorateGeneral for Regional and Urban Policy, http://dx.doi.org/10.2776/461701.

Consip, Italie (2016), Country case: Supplier Training Desks (STDs) in Italy, http://www.oecd.org/governance/procurement/toolbox/search/supplier-training-desks-std-stritaly.pdf (consulté le 24 janvier 2019).

Direction des achats de l'État (DAE) (2018), DAE - Présentation, https://www.economie.gouv.fr/dae/presentation (consulté le 21 novembre 2018).

Direction des affaires juridiques, France (2018), Conseil aux acheteurs - Les guides, https://www.economie.gouv.fr/daj/conseil-acheteurs-guides\#cp (consulté le 19 décembre 2018).

EBRD (2012), Capacity Building in Procurement: A sample assessment in the EBRD region, https://www.ebrd.com/downloads/procurement/project/EBRD Capacity Building Report.pdf (consulté le 25 janvier 2019).

EPSA Groupe (2017), Acheteur public, un métier en pleine transformation, https://www.epsagroupe.com/acheteur-public-un-metier-en-pleine-transformation/ (consulté le 19 décembre 2018).

Gouvernement d'Algérie (2007), Journal Officel de la République algérienne $n^{\circ} 61$, https://services.mesrs.dz/DEJA/fichiers sommaire des textes/162\%20FR.PDF (consulté le 6 novembre 2018).

Gouvernement d'Algérie (2006), Statut général de la fonction publique, https://www.joradp.dz/hfr/dgfp.htm (consulté le 25 janvier 2019).

Gouvernement de France (2017), Répertoire interministériel des métiers de l'État (RIME) | Portail de la Fonction publique, https://www.fonction-publique.gouv.fr/repertoireinterministeriel-des-metiers-de-letat-rime (consulté le 14 décembre 2018).

Gouvernement de Tunisie (2018), Observatoire National des Marchés Publics (ONMP) - Tunisie : Appels d'offres, lois, décrets, arrêtés, http://www.marchespublics.gov.tn/onmp/intervenants/intervenant.php?id=7\&lang=fr\# (consulté le 12 décembre 2018).

Groupe COSIDER (2015), Procédure de passation des commandes.

ICPC (s.d.), Instance Centrale de Prévention de la Corruption, http://www.icpc.ma/wps/portal/lut/p/c4/04 SB8K8xLLM9MSSzPy8xBz9CP0os3h3A9NAFzdTE wN3s0BnA09HJ0 vYOMgQ19 Y 2CbEdFABkSh4!/?WCM GLOBAL CONTEXT=/wps/wcm/connect/internet+icpc fr/icpc/accueil/actualites/ateli er+formation+des+formateurs+22+23+11+2017 (consulté le 18 janvier 2019).

OCDE (2017), Developing Administrative Capacity for Public Procurement in the Slovak Republic : A training action plan for 2016-2019, OCDE, Paris, http://www.oecd.org/governance/procurement/toolbox/search/capacity-public-procurementslovak-republic-training.pdf (consulté le 14 août 2018). 
OCDE (2017), Public Procurement in Peru: Reinforcing Capacity and Co-ordination, Examens de l'OCDE sur la gouvernance publique, Éditions OCDE, Paris, https://dx.doi.org/10.1787/9789264278905-en.

OCDE (2016), Checklist for Supporting the Implementation of OECD Recommendation of the Council on Public Procurement: Balance, OCDE, Paris, http://www.oecd.org/governance/procurement/toolbox/search/Checklist\%2004\%20Balance.pd f (consulté le 13 décembre 2018).

OCDE (2016), Checklist for Supporting the Implementation of OECD Recommendation of the Council on Public Procurement: Capacity, OCDE, Paris, http://www.oecd.org/governance/procurement/toolbox/search/Checklist $\% 2008 \% 20$ Capacity.p df (consulté le 16 août 2018).

OCDE (2016), Public Procurement Training for Bulgaria: Needs and priorities, OCDE, Paris, http://www.oecd.org/governance/procurement/toolbox/search/public-procurement-trainingbulgaria EN.pdf (consulté le 14 août 2018).

OCDE (2016), Roadmap: How to Elaborate a Procurement Capacity Strategy, OCDE, Paris, http://www.oecd.org/governance/ethics/Roadmap-Procurement-Capacity-Strategy.pdf (consulté le 11 septembre 2018).

OCDE (2015), Recommandation du Conseil de l'OCDE sur les marchés publics, OCDE, Paris, http://www.oecd.org/fr/gov/ethique/Recommandation-OCDE-sur-les-marches-publics.pdf (consulté le 16 août 2018).

OCDE (2014), Going Green: Best practices for Green Procurement - CANADA, OCDE, Paris, http://www.oecd.org/gov/ethics/gpp-procurement-Canada.pdf (consulté le 21 janvier 2019).

OCDE (2013), Public Procurement Review of the State's Employees' Social Security and Social Services Institute in Mexico, Examens de l'OCDE sur la gouvernance publique, Éditions OCDE, Paris, https://dx.doi.org/10.1787/9789264197305-en.

OECD (2013), Implementing the OECD Principles for Integrity in Public Procurement : Progress since 2008, OECD Public Governance Reviews, OECD Publishing, Paris, https://dx.doi.org/10.1787/9789264201385-en.

UNESCO (2018), Ressources éducatives libres, https://fr.unesco.org/themes/tic-education/rel (consulté le 18 janvier 2019).

Université de Nottingham, Royaume-Uni (s.d.), Public Procurement Law and Policy LLM/PGDip/PGCert - The University of Nottingham, https://www.nottingham.ac.uk/pgstudy/courses/law/public-procurement-law-and-policyIIm.aspx (consulté le 23 janvier 2019).

UNPCDC (2011), Strengthening Country Procurement Systems: Results and Opportunities, http://siteresources.worldbank.org/INTALBANIA/Resources/Strengthening Procurement Syst ems.pdf (consulté le 16 août 2018).

World Bank (2014), MOOC - Public Procurement Training Courses, https://www.procurementlearning.org/certificate-program-public-procurement-cppp/ (consulté le 18 janvier 2019). 



\section{Examens de l'OCDE sur la gouvernance publique \\ Revue du système de passation des marchés publics en Algérie}

\section{VERS UN SYSTÈME EFFICIENT, OUVERT ET INCLUSIF}

Représentant plus de $20 \%$ du PIB en Algérie, les marchés publics ont un impact considérable sur l'économie du pays, l'efficience du secteur public et sur la confiance des citoyens. Cette revue souligne les forces et les opportunités d'amélioration du système de passation des marchés publics dans un certain nombre de domaines : le cadre institutionnel et règlementaire, la concurrence, la transparence et la digitalisation du système, son intégrité, son efficience, l'utilisation stratégique des marchés publics et le renforcement des capacités des acteurs du système de passation des marchés publics.

Veuillez consulter cet ouvrage en ligne : https://doi.org/10.1787/49802cd0-fr.

Cet ouvrage est publié sur OECD iLibrary, la bibliothèque en ligne de l'OCDE, qui regroupe tous les livres, périodiques et bases de données statistiques de l'Organisation.

Rendez-vous sur le site www.oecd-ilibrary.org pour plus d'informations.

\section{éditionsOCDE}

www.oecd.org/editions
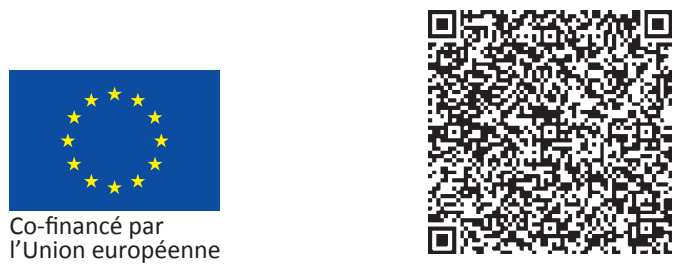

ISBN 978-92-64-32691-0

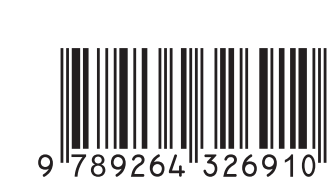

